University of Louisville

ThinkIR: The University of Louisville's Institutional Repository

Electronic Theses and Dissertations

$12-2013$

\title{
Constructing the rod bipolar cell signalplex using animal models of retinal dysfunction.
}

Thomas Andrew Ray 1986-

University of Louisville

Follow this and additional works at: https://ir.library.louisville.edu/etd

\section{Recommended Citation}

Ray, Thomas Andrew 1986-, "Constructing the rod bipolar cell signalplex using animal models of retinal dysfunction." (2013). Electronic Theses and Dissertations. Paper 1188.

https://doi.org/10.18297/etd/1188

This Doctoral Dissertation is brought to you for free and open access by ThinkIR: The University of Louisville's Institutional Repository. It has been accepted for inclusion in Electronic Theses and Dissertations by an authorized administrator of ThinkIR: The University of Louisville's Institutional Repository. This title appears here courtesy of the author, who has retained all other copyrights. For more information, please contact thinkir@louisville.edu. 
CONSTRUCTING THE ROD BIPOLAR CELL SIGNALPLEX USING ANIMAL MODELS

\author{
OF RETINAL DYSFUNCTION
}

\author{
By
}

Thomas Andrew Ray

B.S., Eastern Kentucky University, 2004

\begin{abstract}
A Dissertation
Submitted to the Faculty of the

School of Medicine of the University of Louisville

In Partial Fulfillment of the Requirements
\end{abstract}

For the Degree of

\author{
Doctor of Philosophy \\ Department of Biochemistry and Molecular Biology \\ University of Louisville \\ Louisville, Kentucky
}

December 2013 

CONSTRUCTING THE ROD BIPOLAR CELL SIGNALPLEX USING ANIMAL MODELS OF RETINAL DYSFUNCTION

By

Thomas Andrew Ray

B.S., Eastern Kentucky University, 2004

A Dissertation Approved on

November 15, 2013

By the following Dissertation Committee:

\begin{tabular}{c}
\hline Ronald G Gregg, PhD \\
\hline Maureen A McCall, PhD \\
\hline Brian Wattenberg, PhD \\
\hline Alan Cheng, PhD \\
\hline Richard Feldhoff, PhD
\end{tabular}




\section{ACKNOWLEDGEMENTS}

I would like to thank my mentor, Dr. Ronald Gregg, for his ability to guide and challenge while empowering me with academic freedom. I would like to thank my committee members: Dr. Maureen McCall, Dr. Alan Cheng, Dr. Brian Wattenberg, and Dr. Richard Feldhoff, for their support and guidance. I would like to thank the faculty and staff of the Department of Biochemistry and Molecular Biology at the University of Louisville for shaping my graduate school experience and fostering an enriching environment in which to learn. I would like to thank my parents, Mike and Jean Ray, for instilling me with the work ethic to satisfy my curiosity. I would also like to think my wife, Sara Ray, for taking part in this journey. 


\section{ABSTRACT \\ CONSTRUCTING THE ROD BIPOLAR CELL SIGNALPLEX USING ANIMAL MODELS OF RETINAL DYSFUNCTION}

Thomas A. Ray

November 15, 2013

Glutamate mediated neurotransmission from the rod photoreceptors to rod bipolar cells is critical for vision under low light levels. Disruption of this pathway results in the blinding disorder Congenital Stationary Night Blindness (CSNB), which is hallmarked by the inability to see under dim light. Mouse models of CSNB have been critical to identifying proteins required for glutamate signaling and understanding the mechanism of signaling. Currently, our understanding of rod bipolar signaling is incompletely understood because not all of the protein components of the signalplex have been identified. It was known that the glutamate receptor mGluR6 is coupled to the TRPM1 cation channel, via an unknown $G$ protein mediated mechanism and that regulators of $G$ protein signaling (RGS) are required to terminate signaling. During my graduate work, I identified two new protein components of the rod bipolar cell signalplex that are critical to rod-mediated vision. By mapping the mutation in a novel mouse model of CSNB, I identified Gpr179 as critical to rod bipolar cell signaling. Using immunohistochemistry followed by confocal microscopy and protein interaction assays, I identified GPR179 as a signalplex component. Further, we showed that mutations in GPR179 cause CSNB in humans. I found that GPR179 functions as a hub by interacting with TRPM1 and recruiting the RGS protein complex to the signalplex, which serves to enhance the sensitivity of the system. A report identified mutations in LRIT3 cause CSNB in humans (Zeitz et al., 2013). To determine how LRIT3 contributes to rod vision, I created an $\mathrm{Lrit3}^{--}$mouse model of CSNB and found that LRIT3 is required for localizing TRPM1 to the signalplex. These findings add to our understanding of how the visual signal is passed through the retina, and more specifically, rod bipolar cell signalplex architecture, G protein signaling mechanisms, and the causes of CSNB. 
TABLE OF CONTENTS

PAGE

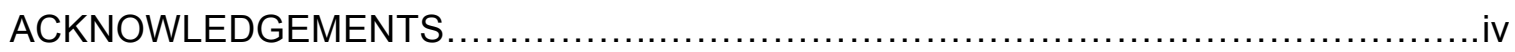

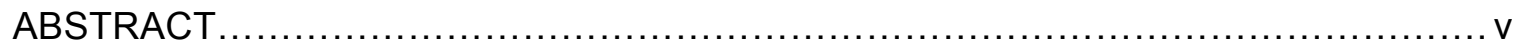

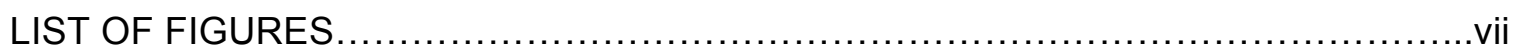

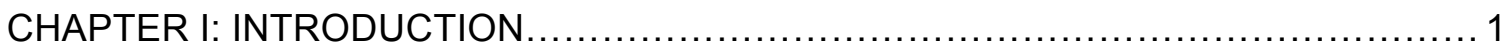

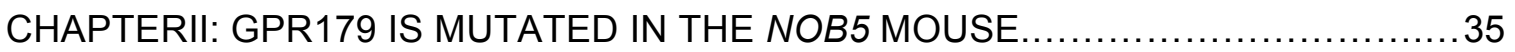

CHAPTER III: CONSTRUCTING THE DEPOLARIZING BIPOLAR CELL

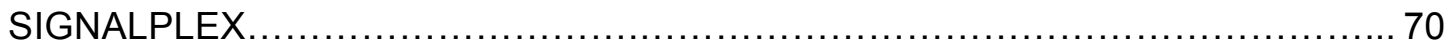

CHAPTER IV: LRIT3 IS REQUIRED FOR NORMAL DEPOLARIZING BIPOLAR CELL

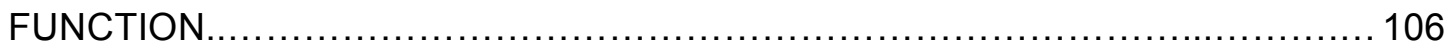

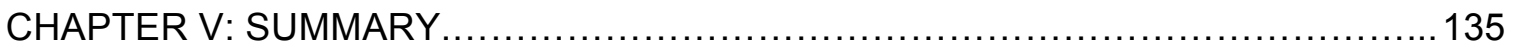

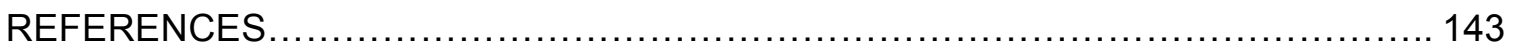

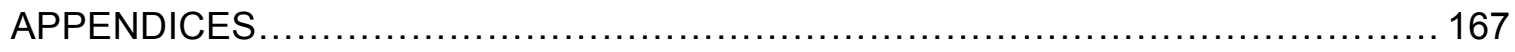

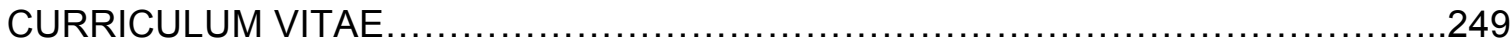




\section{LIST OF FIGURES}

$\begin{array}{ll}\text { FIGURE PAGE } & \text { PAR }\end{array}$

1. Fluorescent microscopy image and schematic of retinal layers ........................

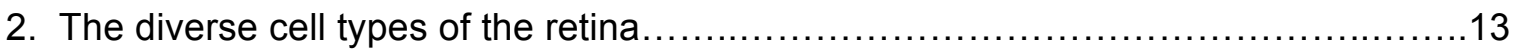

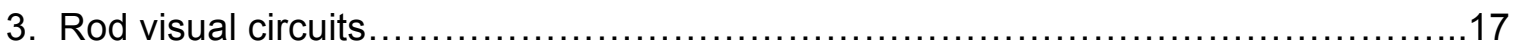

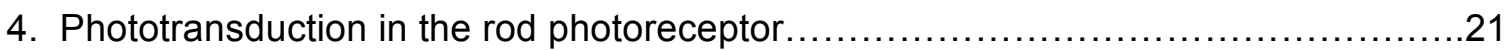

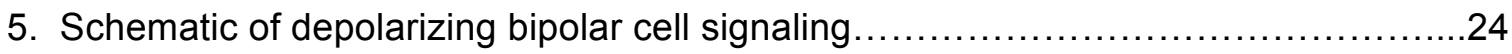

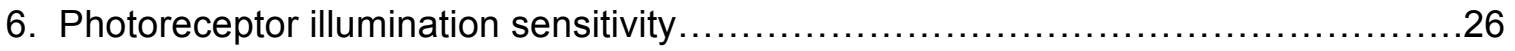

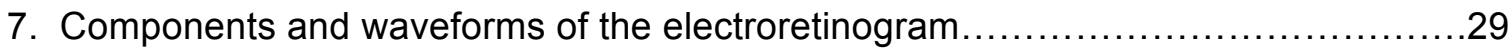

8. Scotopic and photopic ERG of WT and nob5 mice.......................... 37

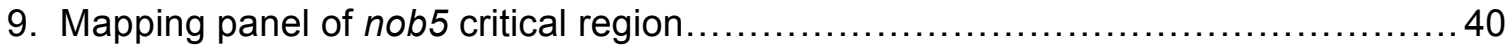

10. A transposable element disrupts Gpr179 expression........................... 43

11. In situ hybridization localizes Gpr179 mRNA expression.........................46

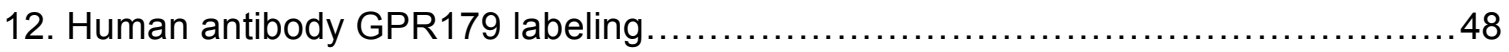

13. GPR179 immunostaining in WT and nob5 retinal slices.........................50

14. GPR179 is expressed post synaptically at the tips of the DBCs $\ldots \ldots \ldots \ldots \ldots \ldots \ldots \ldots \ldots$

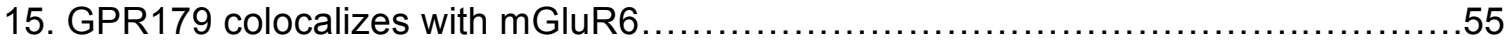

16. Morpholino knockdown of GPR179 in zebrafish................................. 57

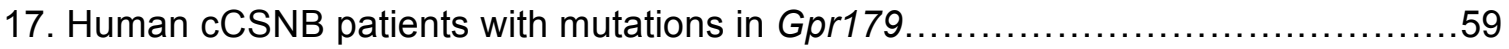

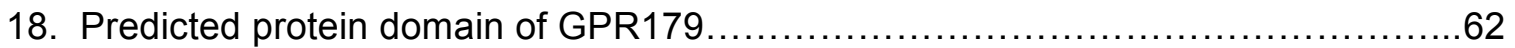

19. GPR179 expression in various mouse models of DBC dysfunction.................74

20. Expression of major signaling components in the $G p r 179^{\text {nob5 }}$ retina................ 76 
21. Signalplex protein expression in various mouse mutants $I \ldots \ldots \ldots \ldots \ldots \ldots \ldots \ldots \ldots \ldots$

22. Signlaplex protein expression in various mouse mutants $I I \ldots \ldots \ldots \ldots \ldots \ldots \ldots \ldots \ldots$

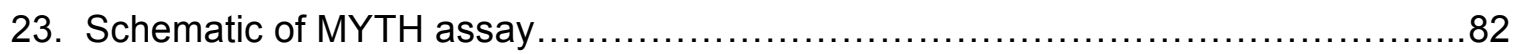

24. Screening Gpr179 bait vectors for use in MYTH system ....................... 86

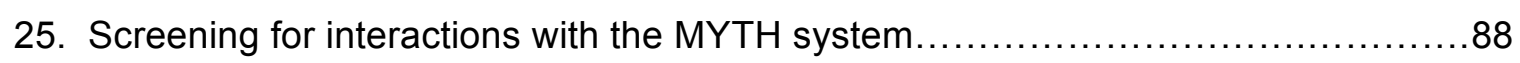

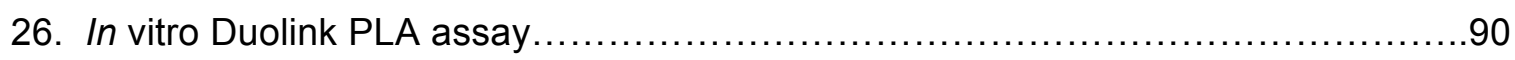

27. GPR179 interacts with mGluR6 and TRPM1 in vitro.......................... 92

28. TRPM1 and GPR179 localize within the Duolink critical radius................... 94

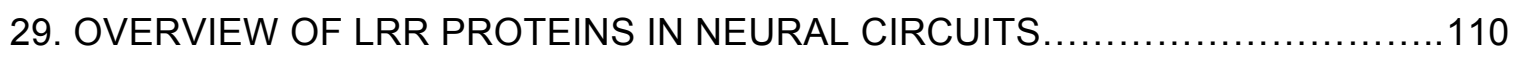

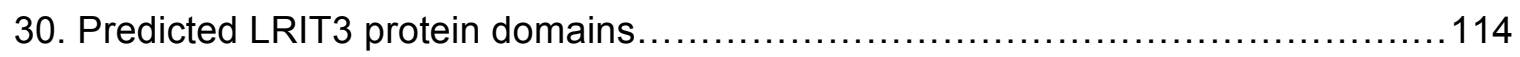

31. Workflow of the generation of a ZFN KO mouse model........................116

32. ZFN mutations disrupt a highly conserved protein-coding region.................119

33. Predicted $L$ rit3 $^{-/}$mice have a no b-wave ERG phenotype....................... 121

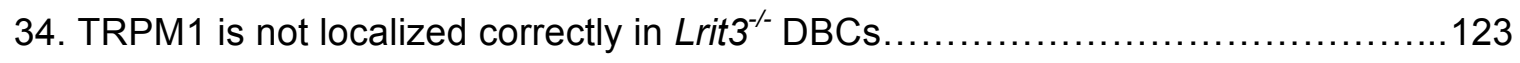

35. GPR179, mGluR6, Gß5, RGS7 and RGS11 localization is normal in Lrit3 ${ }^{-\ldots} \ldots \ldots . .125$

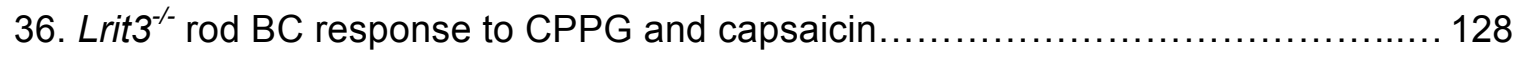

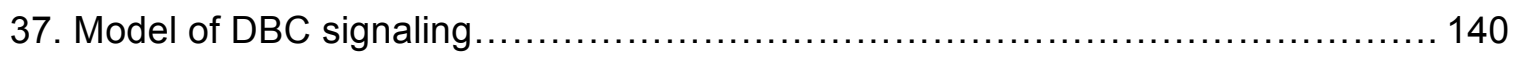




\section{CHAPTER I: INTRODUCTION}

Our ability to visualize the text before us and consistently and accurately convert contrast differences into meaningful symbols is made possible by the retina, a product of over 600 million years of evolution (Lamb et al., 2007). The earliest photosensitive cells that eventually gave rise to the retina consisted of a single cell photoreceptor capable of phototaxis, shadow detection and entrainment of circadian rhythms that gave our ancient ancestors survival advantages (Lamb et al., 2007). This once rudimentary system for detecting changes in ambient light evolved into a highly specialized multicellular tissue capable of image-formation during the Cambrian explosion 540 million years ago (Lamb et al., 2007). In its current form, the human retina has evolved to function over a wide range of light intensities, provide both high sensitivity and high acuity, and carry out complex image processing.

Humans are heavily invested in their visual system to function during daily life and blinding disorders have a dramatic effect on a person's quality of life. Because of this, it is of great interest to understand the circuitry of the retina and the mechanism of communication between retinal neurons in the healthy and diseased state. Retinal structure and function has been studied for decades, but there are still cell types to be discovered, circuits to be identified and receptors that allow cells to communicate to be characterized. Apart from gaining insight about vision, the retina is a great model system for studying general neural signaling and neural development. The accessibility

of the retina along with the ability to easily assess function makes it a great model system to study neuronal function. 


\section{Retina Anatomy}

The vertebrate retina is a light sensitive neural tissue at the back of the eye that consists of various synaptic and nuclear layers juxtaposed between an outer retinal pigmented epithelium (RPE) and an inner limiting membrane (ILM) (Cajal, 1892b). The basic organization of the retina is remarkably similar across vertebrate species (Masland, 1986; Ehinger et al., 1988; Marc et al., 1990; Wassle and Boycott, 1991). The first detailed anatomical view of the mammalian retina was produced by Santiago Ramón y Cajal in 1892 using golgi silver nitrate stained retinas (Cajal, 1892b). He showed the retina consists of three nuclear layers; the outer nuclear layer (ONL), the inner nuclear layer (INL) and the ganglion cell layer (GCL) in which cell bodies reside, and two synaptic layers, the outer plexiform layer (OPL) and the inner plexiform layer (IPL) in which synapses are formed between various retinal neurons (Cajal, 1892b; Kolb, 1970). A simplistic depiction of retinal anatomy is shown in Fig.1.

The photoreceptor cell bodies reside in the ONL and the outer segments of the photoreceptors are apposed to the RPE (Bok, 1993). Bipolar cells (BCs), amacrine cells and horizontal cells reside in the INL and ganglion cells and displaced amacrine cells reside in the GCL (Cajal, 1892a; Cajal, 1892b; Hughes and Wieniawa-Narkiewicz, 1980). In the OPL, rod and cone photoreceptors form synaptic connections with BCs and horizontal cells (Kolb, 1970). The IPL is divided into two different sublamina, A and B. The OFF ganglion cells make contacts with OFF BCs in sublamina $A$ and $O N$ ganglion cells make contacts with ON BCs in sublamina B (Rodieck, 1973; Famiglietti et al., 1977; Nelson et al., 1978; Wassle and Boycott, 1991). The ganglion cell axons form the optic nerve and transmit signals to the visual centers of the brain. Each neuron type plays a specific role in extracting information from the visual environment and packaging that information into a neural code that is sent to the visual centers of the brain for interpretation. 
In addition to the many neurons in the retina, there are three types of glial cells. Glial cells are non-neuronal cells that provide support to neurons. The primary glial cell in the retina is the Müller glial cell, which spans the retina from the RPE to the ILM. Astrocytes envelope retinal vasculature and play an important role in the blood-retinal barrier (Ridet et al., 1997). Microglial cells provide innate immune responses and can migrate to regions of damaged cells or exogenous pathogens (Aloisi, 2001; Lynch, 2009; Zinkernagel et al., 2013).

The major excitatory neurotransmitter in the retina is glutamate and the major inhibitory neurotransmitters are gamma aminobutyric acid (GABA) and glycine. Excitatory and inhibitory neurotransmitters are needed to set up the excitatory vertical pathways through the retina and the lateral inhibitory pathways. These excitatory and inhibitory pathways enable retinal cells to have a center-surround organization. The classical view of center-surround organization is that a ganglion cell is excited or inhibited when a stimulus falls in the middle of its receptive field but the opposite is achieved when a stimulus falls in the surrounding region. Center-surround organization is critical for the extraction of visual features such as object edge detection. 


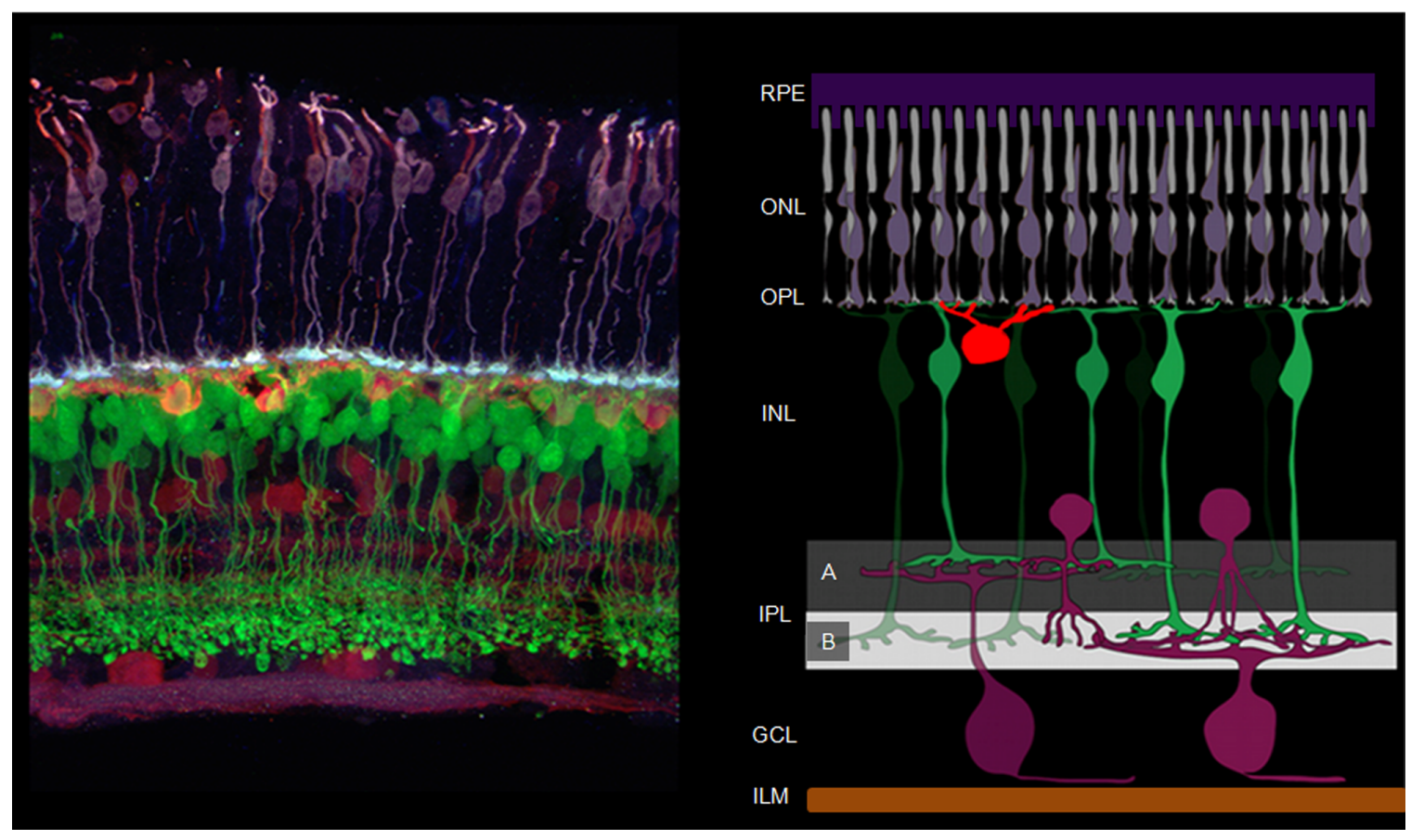

Figure 1: Fluorescent microscopy image and schematic of retinal layers. (Left) Image of mouse retina cross section labeled for cones (purple), horizontal cells (orange), bipolar cells (green), amacrine and ganglion cells (magenta). (Right) Schematic of the retinal layers and the cells that inhabit each layer. The fluorescence microscopy image was adapted and modified from Morgan and Wong (1995) and the retina schematic from http://wonglab.biostr.washington.edu/. 


\section{Anatomy and Physiology of the Retina \\ Retinal Pigment Epithelium}

The RPE is a mono layer of pigmented cells that is apposed to the photoreceptor outer segments (OS) and is present in eyes from simple insects to higher vertebrates (Lamb et al., 2007). The RPE contains long apical microvilli that surround the outer segments of the photoreceptors (Bok, 1993; Strauss, 2005). Interactions between the RPE and photoreceptor OS are essential for visual function (Steinberg, 1985; Bok, 1993; Strauss, 2005; Sparrow et al., 2010). The RPE is responsible for delivering nutrients to the photoreceptors and uptake of metabolic end products secreted by the photoreceptors. It is also responsible for the endocytosis of the continuously shed photoreceptor outer segments (Young, 1967; Steinberg, 1985; Bok, 1993; Dornonville de la Cour, 1993; Hamann, 2002). In addition to the RPE providing metabolic support for the photoreceptors, the highly pigmented cells absorb light that passes through the retina. This helps preserve visual acuity by preventing light from scattering back through the retina (Bok, 1993; Boulton and Dayhaw-Barker, 2001).

The RPE plays a major role in supplying the photoreceptors with the visual photopigment, retinal. Retinal (Vitamin A) is a photosensitive aldehyde that undergoes isomerization when it absorbs a photon and it is bound by the opsin G protein-coupled receptors of the photoreceptors. The active form of retinal (11-cis retinal) is used by the photoreceptor opsins for the photoresponse. Once 11-cis retinal absorbs a photon it is converted to the inactive form (all-trans retinal) and is released from the photoreceptors and taken into the RPE where it undergoes isomerization into the active form and subsequent release to be used again by the photoreceptors (Baehr et al., 2003; Besch et al., 2003; Thompson and Gal, 2003).

\section{Photoreceptors}


The photoreceptors are the most abundant cells in the retina (Curcio et al., 1990). Light enters the eye through the iris and passes through all of the neural layers before it reaches the photoreceptors. Individual photons are absorbed by photopigments in the outer segments of the photoreceptors and the photoresponse is initiated. The outer segments contain tightly packed disk membranes in which the photopigments reside (Sjostrand, 1953). There are two different classes of photoreceptors, the rod and the cone photoreceptors, named for the 3-dimensional shapes of their outer segments (Schultze, 1866). In the human retina there are approximately 120 million rods and 6.4 million cones or $95 \%$ of the photoreceptors are rods and $5 \%$ cones (Osterberg, 1937; Curcio et al., 1990). The mouse retina contains a higher percentage of rods, with rods making up $\sim 97 \%$ of the photoreceptors (Carter-Dawson and LaVail, 1979). In the primate retina there is one type of rod photoreceptor but three different types of cones: L-type, M-type and S-type, which differ in the type of opsin that is expressed in the outer segments (Brown and Wald, 1963). The mouse retina only contains two types of cones, S-type (Jacobs et al., 1991) and coexpressing S/M-type (Rohlich et al., 1994; Applebury et al., 2000).

The inner segments of the photoreceptors are located in the ONL and contain the photoreceptor nuclei. The axon terminals of photoreceptors form synapses in the OPL with bipolar cells and horizontal cells (Cajal, 1892b; Kolb, 1970). The axon terminals of the photoreceptors contain a specialized structure termed a ribbon synapse (Sterling and Matthews, 2005). The ribbon synapse facilitates tonic neurotransmitter vesicle release and is capable of dynamic adjustment of release over a wide range of light intensities (Schmitz, 2009). The cone photoreceptor axon terminal is termed a cone pedicle, which is a wide synaptic terminal containing between 20 and 50 invaginations, each harboring synaptic ribbons (Wassle, 2004) and are filled by BC and horizontal cell dendrites (Kolb, 1970). The cone pedicle is one of the most complex synapses in the 
central nervous system (CNS), making as many as 500 post synaptic contacts (Haverkamp et al., 2000). The rod axon terminal is called a rod spherule and contains a single ribbon synapse with one invaginating rod $\mathrm{BC}$ and two horizontal cell dendrites (Kolb, 1970).

\section{Bipolar Cells}

$\mathrm{BCs}$ are the interneurons of the retina that connect photoreceptors to ganglion cells. The $\mathrm{BC}$ dendrites project into the $\mathrm{OPL}$ and the $\mathrm{ON}$ BCs form invaginating synapses with the photoreceptor axon terminals and the OFF BC dendrites make synaptic contacts with cone pedicles. Each bipolar cell makes synaptic connections with one to several different photoreceptors. Overall, the mouse retina has a ratio of approximately 10 photoreceptors per bipolar cell (Jeon et al., 1998). The axons of the BCs project into the IPL and make synaptic connections with amacrine cells and ganglion cells (Cajal, 1892b; Rodieck, 1973; Famiglietti and Kolb, 1975; Nelson et al., 1978; Dacheux and Raviola, 1986; Wassle and Boycott, 1991).

The visual response is split into two different pathways in the OPL, the ON (depolarizing) and the OFF (hyperpolarizing) pathways (Saito and Kaneko, 1983; Attwell et al., 1987; Nawy and Jahr, 1991; Masu et al., 1995). These pathways are mediated by the response of the BCs to a decrease in glutamate release from the photoreceptors. Cones form synapses with both $\mathrm{ON}$ and OFF BCs. ON BCs (DBCs) depolarize in response to light increments and OFF BCs (HBCs) hyperpolarize in response to light increments (Saito and Kaneko, 1983; Attwell et al., 1987; Nawy and Jahr, 1991; Masu et al., 1995). The rod photoreceptors synapse with a single class of ON BCs, the rod BCs, which does not signal directly to ganglion cells (Cajal, 1892b; Boycott et al., 1969; Dacheux and Raviola, 1986).

The difference in the ON and OFF type of BCs is accounted for by the type of glutamate receptor the BC expresses on its dendrites. The OFF BCs or HBCs utilize 
ionotropic glutamate receptors of the AMPA/Kainate type (Slaughter and Miller, 1983) and the ON BCs or DBCs use the metabotropic glutamate receptor 6 (mGluR6) (Masu et al., 1995). BCs that produce a sustained or transient response are differentiated by the expression of rapidly or slowly inactivating glutamate receptors (Awatramani and Slaughter, 2000; DeVries, 2000).

In addition to BCs being divided into ON and OFF classes by physiology, they are also classified based on morphology. In the mammalian retina twelve different types of bipolar cells have been identified based on morphology (Fig. 2) (Ghosh et al., 2004; MacNeil et al., 2004; Wassle et al., 2009; Helmstaedter et al., 2013). Eleven of the BC types make contacts with cones and only the rod BCs make contacts with rods. Each of the different classes of cone BCs is thought to parse specific information from the cone's output and relay that information to the ganglion cells (Masland, 2012). All of the ON bipolar cells stratify in the inner half (sublamina b) of the IPL and OFF bipolar cells stratify in the outer half (sublamina a) of the IPL (Famiglietti et al., 1977; Nelson et al., 1978).

\section{Horizontal Cells}

Horizontal cells form lateral connections in the OPL. At least two types of horizontal cells have been described in the primate retina, the $\mathrm{HI}$ and $\mathrm{HII}$ (Fig. 2) (Kolb et al., 1980; Kolb et al., 1994). The HI horizontal cell has two distinct arbors. The dendritic arbor associated with the cell soma makes synaptic contacts with cone pedicles and the remote axonal arbor makes synaptic connections with rods (Kolb, 1970; Masland, 2011). The HII horizontal cell has a broad, radial dendritic spread that makes synaptic contacts with cones (Kolb et al., 1980; Ahnelt and Kolb, 1994b, a; Masland, 2011). The mouse retina only contains the HI type horizontal cell (Peichl and Gonzalez-Soriano, 1994).

The horizontal cells form inhibitory contacts with rod spherules and cone pedicles

and are electrically coupled to one another by gap junctions (Kolb, 1970; Klaassen et al., 
2011; Sun et al., 2012). It also has been suggested they form inhibitory connections with BC dendrites (Herrmann et al., 2011). Horizontal cells have center surround organization and are thought to be responsible for setting up receptive fields in the retina through lateral inhibitory synapses (Werblin and Dowling, 1969; Mangel and Miller, 1987; Mangel, 1991). In addition to inhibition, horizontal cells provide lateral gain control to the outer retina by averaging the brightness within a local region and subtracting a proportional value from rod and cone output (Klaassen et al., 2011). This ensures that the signal passed through the retina will be within the operating range of downstream neurons (VanLeeuwen et al., 2009; Masland, 2011).

\section{Amacrine Cells}

Amacrine cells reside in the INL and provide lateral connections in the IPL. Amacrine cells are the most diverse cell type in the retina with $\sim 50$ distinct types being identified by morphology and physiology in the mouse (Fig. 2) (MacNeil and Masland, 1998; MacNeil et al., 1999; Lin and Masland, 2006). They can be organized into four

general classes based on their dendritic spread; narrow field $(30-150 \mu m)$, small-field $(150-300 \mu \mathrm{m})$, medium-field $(300-500 \mu \mathrm{m})$, and wide-field $(>500 \mu \mathrm{m})$ (Kolb and Nelson, 1981). Amacrine cells are also classified based on their stratification within the IPL (Mariani, 1990; Kolb et al., 1992).

The functional roles of amacrine cells in the retina are as diverse as their structures. The starburst amacrine cells serve to provide direction selectivity to a subset of ganglion cells (Euler et al., 2002; Fried et al., 2005). The All amacrine cells serve as a link between the rod BC and the ganglion cells (Strettoi et al., 1994; Trexler et al., 2005). The A17 amacrine cells make reciprocal synapses with rod BCs and provide feedback inhibition onto the rod BC axon terminal (Nelson and Kolb, 1985). However, the majority of amacrine cells still have unresolved functions.

\section{Ganglion Cells}


Ganglion cells are the output neurons of the retina and their axons exit the retina through the optic disc and bundle together to form the optic nerve. There are approximately 15 to 20 different types of ganglion cells in the mammalian retina based on morphology and physiology (Fig 2) (Masland, 2001a; Rockhill et al., 2002; Wassle, 2004; Masland, 2011). Each type of ganglion cell is responsible for sending a unique feature of the visual field to specific retinorecipient nuclei in the brain (Roska and Werblin, 2001; Berson, 2008).

The predominant type of ganglion cell in the human retina is the midget ganglion cell which contains a single dendrite extending from the cell soma and accounts for $\sim 80 \%$ of ganglion cells in the retina (Perry et al., 1984). Midget ganglion cells receive input from one cone photoreceptor in the central retina and are capable of relaying color information (Wiesel and Hubel, 1966; Valois, 1973; Lennie, 1984). Parasol ganglion cells have dendritic trees much larger than the midget cells and they receive input from several photoreceptors of mixed spectral input, disabling them from relaying color information (Schiller and Logothetis, 1990; Wassle and Boycott, 1991). The midget and parasol ganglion cells are the predominant ganglion cell types in the primate retina (Schiller and Logothetis, 1990; Merigan and Maunsell, 1993; Dacey, 2004).

There are several other types of ganglion cells, many of which we do not understand their role in vision. Direction selective ganglion cells (DSGCs) respond preferentially to object movement in one of four directions, posterior, anterior, superior and inferior (Oyster and Barlow, 1967; Elstrott et al., 2008; Briggman et al., 2011; Sun et al., 2011). Their discovery was quite revolutionary because it showed retinal neurons were capable of complex visual processing, previously thought to be carried out only in the higher visual centers of the brain (Hubel and Wiesel, 1959; Barlow and Hill, 1963; Barlow et al., 1964). 
Apart from the photoreceptors, the only other known photosensitive cells in the retina are the intrinsically photosensitive retinal ganglion cells (ipRGCs). The ipRGCs express the melanopsin photopigment on their dendrites and cell soma. ipRGCs are responsible for non image forming light responses such as circadian photoentrainment, the pupillary light reflex and light suppression of locomotor activity (reviewed in (Sand et al., 2012)). There is emerging evidence that ipRGCs have roles in image formation and are responsible for pattern recognition (Ecker et al., 2010; Hicks, 2011). The ipRGCs project to several different regions of the brain. Some projections target the suprachiasmatic nucleus of the hypothalamus, which helps the solar cycle synchronize with circadian rhythms (Gooley et al., 2001; Berson et al., 2002; Berson, 2003; Hirota and Fukada, 2004). Another target is the intergeniculate leaflet, which also helps with circadian photoentrainment (Harrington, 1997; Hattar et al., 2002; Morin et al., 2003). A third connection is made to the olivary pretectal nucleus, which mediates the pupillary light reflex (Trejo and Cicerone, 1984; Clarke and Ikeda, 1985; Hattar et al., 2002; Morin et al., 2003).

\section{Müller Cells}

Müller cells are radial glial cells and are the primary glial cells in the retina. They span the entire thickness of the retina and provide light guidance, structural support and nutrient support for their associated neurons (Bringmann et al., 2006; Agte et al., 2011; Reichenbach and Bringmann, 2013). Müller cells orient in the direction of incoming light in the retina and behave as optical fibers to guide light to the photoreceptors (Franze et al., 2007). It has been demonstrated in retinal slices that a thin laser beam traverses all retinal layers if it hits a Müller cell endfoot, but significant intraretinal scattering is observed if the laser hits an area devoid of Müller cells (Agte et al., 2011). In the human retina there is approximately one Müller cell per cone photoreceptor allowing maximum possible resolution (Agte et al., 2011; Reichenbach and Bringmann, 2013). There are 
several rod photoreceptors per Müller cell that optimizes for maximum sensitivity to light (Agte et al., 2011; Reichenbach and Bringmann, 2013).

Each Müller cell provides a structural core that encapsulates a column of retinal neurons (Hollander et al., 1991; Reichenbach and Robinson, 1995). The structural core not only ensures the delivery of light to associated photoreceptors, but Müller cells have a symbiotic relationship with retinal neurons ensuring their viability. Müller cells produce and secrete neurotransmitter precursors to be taken up by retinal neurons (Pow and Crook, 1996; Bringmann et al., 2009). They take up glutamate in the inner retina contributing to rapid removal of glutamate and termination of the excitation (Higgs and Lukasiewicz, 1999; Matsui et al., 1999). Müller cells also protect retinal neurons from reactive oxygen species, which are frequently generated in the retina from light and oxygen consumption. In response to oxidative stress Müller cells secrete glutathione, an antioxidant, to protect the retinal neurons (Schutte and Werner, 1998). Müller cells also serve a critical role in ensuring photoreceptor viability and retinal ion homeostasis (Reichenbach and Bringmann, 2013). 


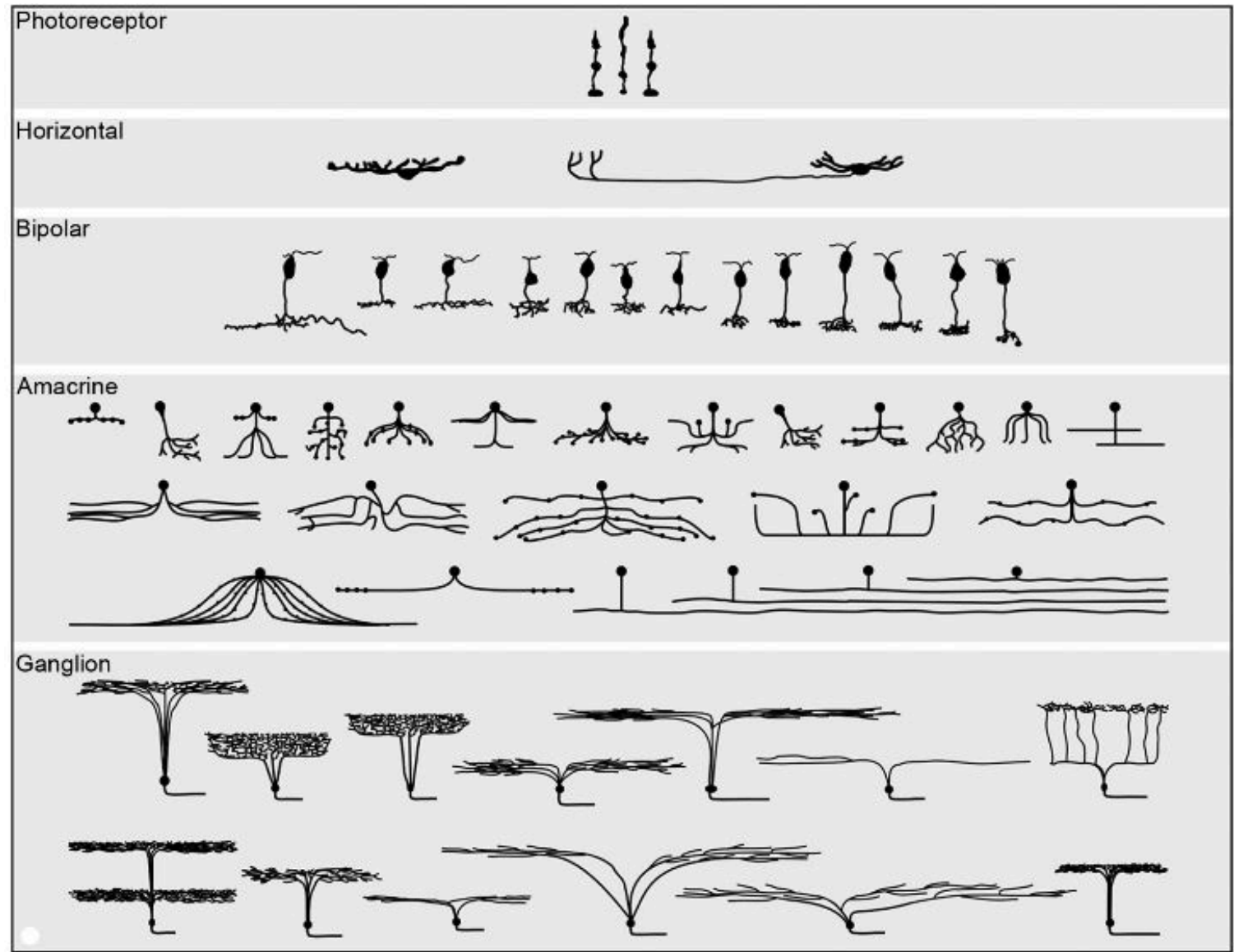

Figure 2: The diverse cell types of the retina. Shown are representative drawings of the many different types of cells found in mammalian retinas. Adapted from (Masland, 2001b). 


\section{Convergence and Divergence}

Retinal cell type distribution across the retina plays an important role in shaping the visual field of animals. Cell type distribution reflects the lifestyle of an animal and varies across species and within species with different lifestyles, allowing the retina to capture information most critical to the animal's survival (Hughes et al., 1977; Stone, 1983; Talbot and Marshall, 2011). Nocturnal plains dwelling animals typically have a horizontal band extending naso-temporal across the retina with high ganglion cell density (Hughes, 1971; Provis, 1979). This "visual streak" is thought to allow detection of moving objects in the periphery. In contrast, most predatory animals, including humans, tend to have photoreceptors and ganglion cells concentrated around the central retina resulting in higher acuity vision (Hughes, 1975; Stone, 1978; Wassle et al., 1989).

Human visual acuity, color vision and sensitivity are the result of the cell type distributions of the fovea and the connections they make (Wassle et al., 1989; Curcio and Hendrickson, 1991). Near the center of the retina is a yellow-pigmented region, known as the macula. The yellow pigmentation is due to the presence of the carotenoids lutein and zeaxanthin (Beatty et al., 1999; Beatty et al., 2004), which absorb short wavelength light and provide a level of protection against short wavelength light damage beyond what is provided by the lens (Snodderly et al., 1984a; Snodderly et al., 1984b). Within the macula is the fovea, consisting of the highest density of cone photoreceptors and lowest density of rods and is devoid of ganglion cell bodies and retinal vessels to improve visual acuity (Curcio and Hendrickson, 1991; Provis et al., 1998). Primates are the only mammals that possess a fovea, although it has evolved independently in some non-mammalian vertebrates (Inzunza et al., 1991; Moore et al., 2012).

When a signal is passed through the retina it can undergo convergence and divergence at each synaptic layer. The convergence of signal comes from several 
photoreceptors pooling their input onto a single ganglion cell. The rod system has highest convergence, which increases the sensitivity of the system at the cost of visual acuity by pooling the input from a large area of the retinal surface. In the cat, as many as 75,000 rods connect to 5,000 rod BCs which connect to 250 All amacrine cells to a single alpha ganglion cell (Wassle et al., 1989). The cone pathway through the retina is less convergent as a whole and the low convergence in the fovea accounts for the high visual acuity. The other factor increasing visual acuity is the ability of cones to pack in close proximity. In the fovea, cones are primarily connected to midget bipolar cells, which connect to midget ganglion cells, setting up a 1:1 cone to ganglion cell ratio (Kolb and Dekorver, 1991). In general, convergence is greatest in the outer retina and lowest in the central retina (Schein, 1988; Curcio et al., 1991; Wa"ssle et al., 1994). The cell topography of the retina allows humans to have high acuity and good color vision at the center of the visual field and high sensitivity and reduced spatial resolution in the periphery.

\section{Rod Visual Circuit}

The rod visual circuit is believed to have developed evolutionarily after the cone pathway (Lamb, 2009). Electron microscopy (EM) revealed that rod bipolar cells do not make direct synaptic contact with ganglion cells. Instead, they make synaptic contacts with a specific class of amacrine cells, the All amacrine cell (Kolb and Famiglietti, 1974; Famiglietti and Kolb, 1975; Strettoi and Masland, 1996) and utilized the preexisting cone BCs to connect to GCs (Strettoi et al., 1992)..

The Rods can transmit their signal to the ganglion cells through two different pathways (Fig. 3). In the primary rod pathway, rods signal to rod BCs, which make synaptic contacts with All and A17 amacrine cells (Famiglietti and Kolb, 1975; Dacheux and Raviola, 1986; Raviola and Dacheux, 1987). The A17 amacrine cells provide GABAergic feedback inhibition onto the rod BC synapses to modulate their output (Kolb 
and Nelson, 1983; Dacheux and Raviola, 1986; Raviola and Dacheux, 1987; Protti and Llano, 1998; Zhang et al., 2002; Chavez and Diamond, 2008). The All amacrine cell is connected with other Alls and the axon terminals of cone ON BCs by gap junctions (Famiglietti and Kolb, 1975; Strettoi et al., 1992; Strettoi et al., 1994; Mills and Massey, 1995; Veruki and Hartveit, 2002; Petrides and Trexler, 2008). It is through the cone ON BCs, via the All, that the rod signal reaches the ON ganglion cell (Kolb, 1979). The Alls also make inhibitory synapses onto select OFF BC axon terminals and OFF ganglion cells, allowing the rod signal to integrate into the ON and OFF pathways (Tsukamoto et al., 2001; Murphy and Rieke, 2008; Munch et al., 2009). In the secondary rod pathway the rod photoreceptors are connected to the cone photoreceptors through gap junctions and the cone ON BCs are used to transmit the signal to the ganglion cells (Raviola and Gilula, 1973; Nelson, 1977; Schneeweis and Schnapf, 1995). 


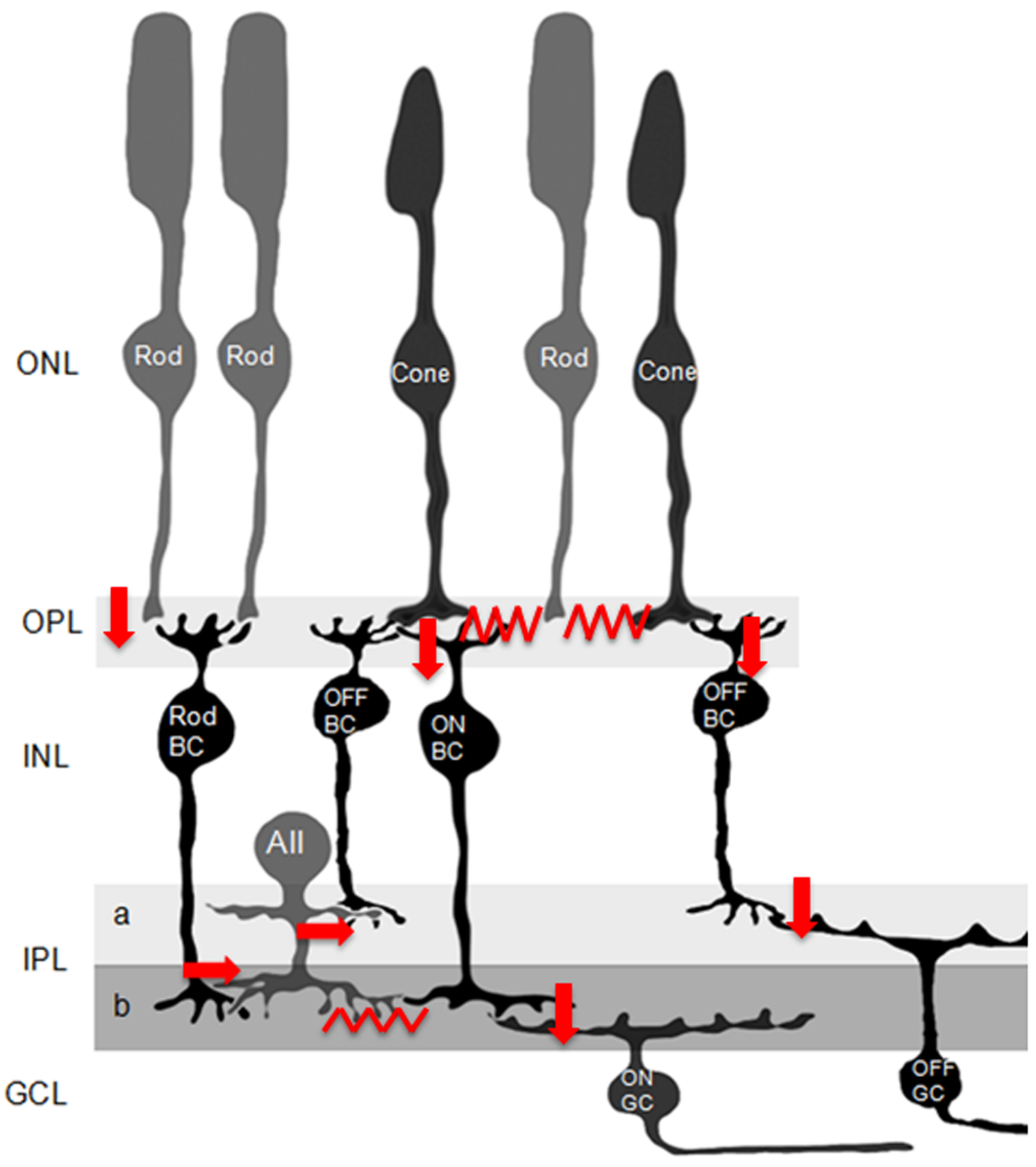

Figure 3: Rod Visual Circuits. The primary rod pathway: rod photoreceptors synapse with the rod BCs, the rod BCs transmit signal to the All amacrine cell, the All amacrine cell is coupled to the cone ON BCs through gap junctions or the OFF $B C$ axon terminals through glycinergic synapses, the cone ON BC signal to the ON ganglion cell (GC) and the OFF BC signal to the OFF GC. Secondary rod pathway: the rods feed directly into cones via gap junctions, the signal is then passed through the cone $\mathrm{ON}$ and OFF BCs to the ganglion cells. 


\section{Phototransduction}

Phototransduction is the process by which a light signal is converted into an electrical signal, which is passed through the retina by the retinal neurons. Phototransduction begins with the absorption of photons by 11-cis-retinal bound to one of the various retinal opsins. Opsins are light sensitive $G$ protein-coupled receptors (GPCRs) and each type of photoreceptor expresses a specific type of opsin. The rod photorecptors express rhodopsin (RHO) and are most sensitive to $498 \mathrm{~nm}$ light, the Ltype cones express long-wave-sensitive opsin 1 (OPN1LW) and are most sensitive to $564 \mathrm{~nm}$ light, the M-type cones express medium-wave-sensitive opsin 1 (OPN1MW) and are most sensitive to $533 \mathrm{~nm}$ light and the S-type cones express short-wave-sensitive opsin 1 (OPN1SW) and are most sensitive to $437 \mathrm{~nm}$ light (Brown and Wald, 1963).

The ligand of the visual pigments of most vertebrates is 11-cis-retinal (Dartnall and Lythgoe, 1965). Absorption of a photon causes a conformation change in the opsin bound chromophore 11-cis-retinal (Wald, 1968). The conformation change from 11-cisretinal to all-trans retinal subsequently activates the associated opsin (Wald, 1968). Activated opsin can then bind and activate the heterotrimeric $G$ protein, transducin, resulting in a GDP for GTP exchange on $\mathrm{Ga}_{t}$ (reviewed in Chen (2005)). Active $\mathrm{Ga}_{\mathrm{t}}$ binds and activates the cyclic guanosine monophosphate (cGMP) phosphodiesterase (PDE), which is anchored to the disk membrane by isoprenylation (Anant et al., 1992; Qin and Baehr, 1994). Activated PDE catalyzes the hydrolysis of cGMP, the second messenger in phototransduction (Zhang and Cote, 2005; Lugnier, 2006; Conti and Beavo, 2007). The decrease in cGMP causes cyclic nucleotide gated (CNG) ion channels to close, resulting in hyperpolarization of the cell (Fesenko et al., 1985; Haynes et al., 1986; Zimmerman and Baylor, 1986; Matthews, 1987; Haynes and Yau, 1990).

Deactivation of phototransduction is the result in deactivation of activated opsins and transducin. Deactivation of opsin begins with phosphorylation of 6 to 7 amino acids 
on the cytoplasmic carboxy terminus (Hurley et al., 1998; Mendez et al., 2000; Kennedy et al., 2004) and is completely inhibited when arrestin binds the phosphorylated cytoplasmic domain and inhibits interaction with transducin (Xu et al., 1997; Chen et al., 1999; Mendez et al., 2000; Doan et al., 2009). The intrinsic rate of GaGTP to GDP conversion is relatively slow and is accelerated by interaction with RGS9 (He et al., 1998). RGS9 is a part of a multi-protein complex that includes R9AP (membrane anchor) and Gß5 (Hu and Wensel, 2002). Hydrolyzing GaGTP to GaGDP inhibits PDE activation and allows levels of cGMP to recover and effectively gate the CNG ion channels open.

Deactivation of phototransduction in rods is slower ( $200 \mathrm{~ms})$ than the activation steps ( 130 ms) (Krispel et al., 2006; Nikonov et al., 2006; Arshavsky and Burns, 2012). This is an important concept because the bipolar cell light response is similar to the deactivation of a $G$ protein cascade and not the activation. In mouse rod photoreceptors, the RGS9 complex rate limits the deactivation of the photoresponse (Krispel et al., 2006). Overexpression of the RGS complex results in a faster photoresponse recovery ( $74 \mathrm{~ms}$ ) (Krispel et al., 2006; Chen et al., 2010; Gross and Burns, 2010). In cones, which express more RGS proteins than rods (Cowan et al., 1998; Zhang et al., 2003), deactivation of rhodopsin becomes the rate-limiting step in photoresponse deactivation (Gross and Burns, 2010; Matthews and Sampath, 2010).

Hyperpolarization of the photoreceptor modulates the L-type voltage gated calcium channel (L-VGCC) and results in channel closure (Katz and Miledi, 1967; Smith and Augustine, 1988; Schmitz and Witkovsky, 1997; Bech-Hansen et al., 1998). The reduction in calcium entry decreases the release of the neurotransmitter glutamate into the synaptic cleft, in a stimulus intensity dependent manner (Trifonov, 1968a; Penn and Hagins, 1969; Ball and Gregg, 2002; Barnes and Kelly, 2002). Photoreceptors release neurotransmitter in a graded manner with a linear relationship to intracellular calcium 
levels (Thoreson et al., 2004; Heidelberger et al., 2005; Thoreson, 2007). RIBEYE, the main protein component of ribbon synapses, forms complexes with proteins that regulate L-VGCCs to tightly correlate synaptic vesicle release to calcium entry (Schmitz et al., 2000; Alpadi et al., 2008; Haeseleer, 2008). 


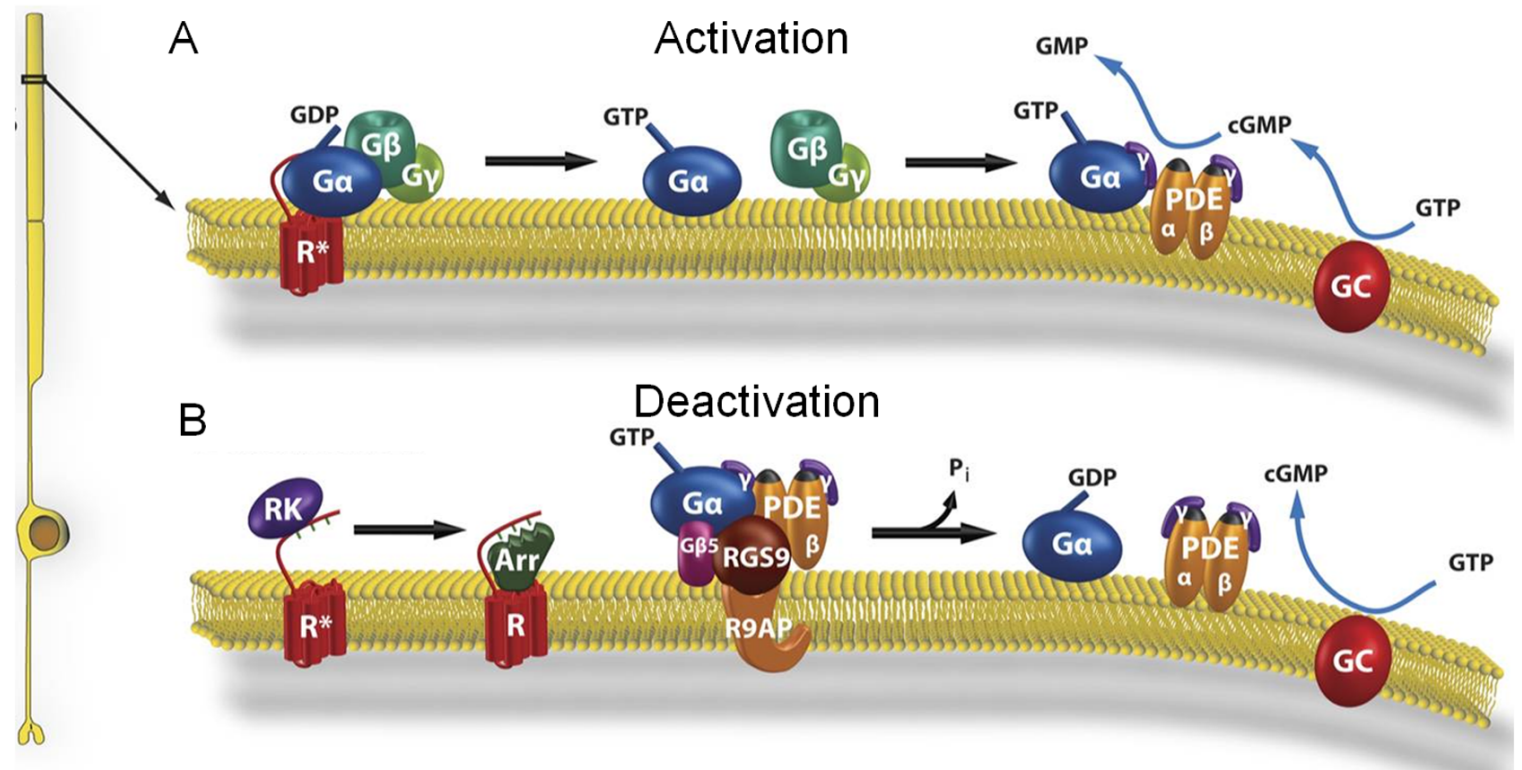

Figure 4: Phototransduction in the rod photoreceptor. (A) Activation of rhodopsin by a single photon causes $G$ protein activation. Active $G \alpha$ binds and activates PDE, allowing it to hydrolyze cGMP. cGMP gated ion channels then open causing the cell to hyperpolarize. (B) Adapted from Arshavsky and Burns (2012) 


\section{ON Bipolar Cell signaling}

BCs are hypothesized to have evolved from photoreceptors due to the fact they share many common features including; microtubule arrangement, an output synapse with similar structure containing a ribbon synapse, and a similar $G$ protein signal transduction cascade (Lamb et al., 2007). While there are many similarities between these cell types, the ON BC signaling cascade is less well understood and it is emerging that the $\mathrm{ON} \mathrm{BC}$ signaling cascade is markedly different from the signaling mechanism in the photoreceptors (Peachey et al., 2012b; Shen et al., 2012).

Signaling from the photoreceptors to the bipolar cells requires glutamate receptors at the dendritic tips of the bipolar cells. The ON bipolar cells express the metabotropic glutamate receptor 6 (mGluR6) and the OFF bipolar cells express different variations of ionotropic glutamate receptors (iGluR) of AMPA and kainate types (Slaughter and Miller, 1981; Saito and Kaneko, 1983; Masu et al., 1995; Puller et al., 2013). A decrease in glutamate release at the photoreceptor terminals cause the OFF BCs to hyperpolarize due to closure of the iGluR cation channel (Kaneko and Saito, 1983; Saito and Kaneko, 1983). The ON BCs depolarize in response to a reduction in glutamate by opening a non-specific cation channel, TRPM1, linked to the mGluR6 glutamate receptor (Slaughter and Miller, 1981; Masu et al., 1995; Morgans et al., 2009; Shen et al., 2009; Koike et al., 2010b; Morgans et al., 2010).

In the dark, photoreceptors tonically release glutamate from their axon terminals into the synaptic cleft (Trifonov, 1968b; Copenhagen and Jahr, 1989; Ayoub and Copenhagen, 1991). Glutamate binding to mGluR6 activates the receptor, releasing the active heterotrimeric $\mathrm{G}$ proteins $\mathrm{Ga}_{\circ}$ and $\mathrm{G} \beta 3 \gamma 13$ dimer (Dhingra et al., 2000; Huang et al., 2003; Okawa et al., 2010; Dhingra et al., 2012). The activation of the G proteins results in the closing of the TRPM1 cation channel by an unknown mechanism (Morgans et al., 2009; Shen et al., 2009; Koike et al., 2010a; Koike et al., 2010b). When the 
photoreceptors hyperpolarize in response to light increments, glutamate release into the synaptic cleft is decreased (reviewed in Stryer (1991)). The reduction in glutamate bound by mGluR6 causes a decrease in activated $G$ proteins allowing the TRPM1 channel to open and the cell to depolarize (Audo et al., 2009; Morgans et al., 2009; Shen et al., 2009; Koike et al., 2010b; Morgans et al., 2010). The RGS proteins G $\beta 5$, RGS7 and -11 are thought to be critical in deactivating the $G$ proteins allowing the TRPM1 cation channel to open (Rao et al., 2007; Cao et al., 2012). Several other proteins with unidentified functions also are critical to normal cascade function and include nyctalopin, GPR179 and LRIT3 (Gregg et al., 2003; Audo et al., 2012; Peachey et al., 2012b; Zeitz et al., 2013a). Figure 5 shows a schematic of DBC signaling. 


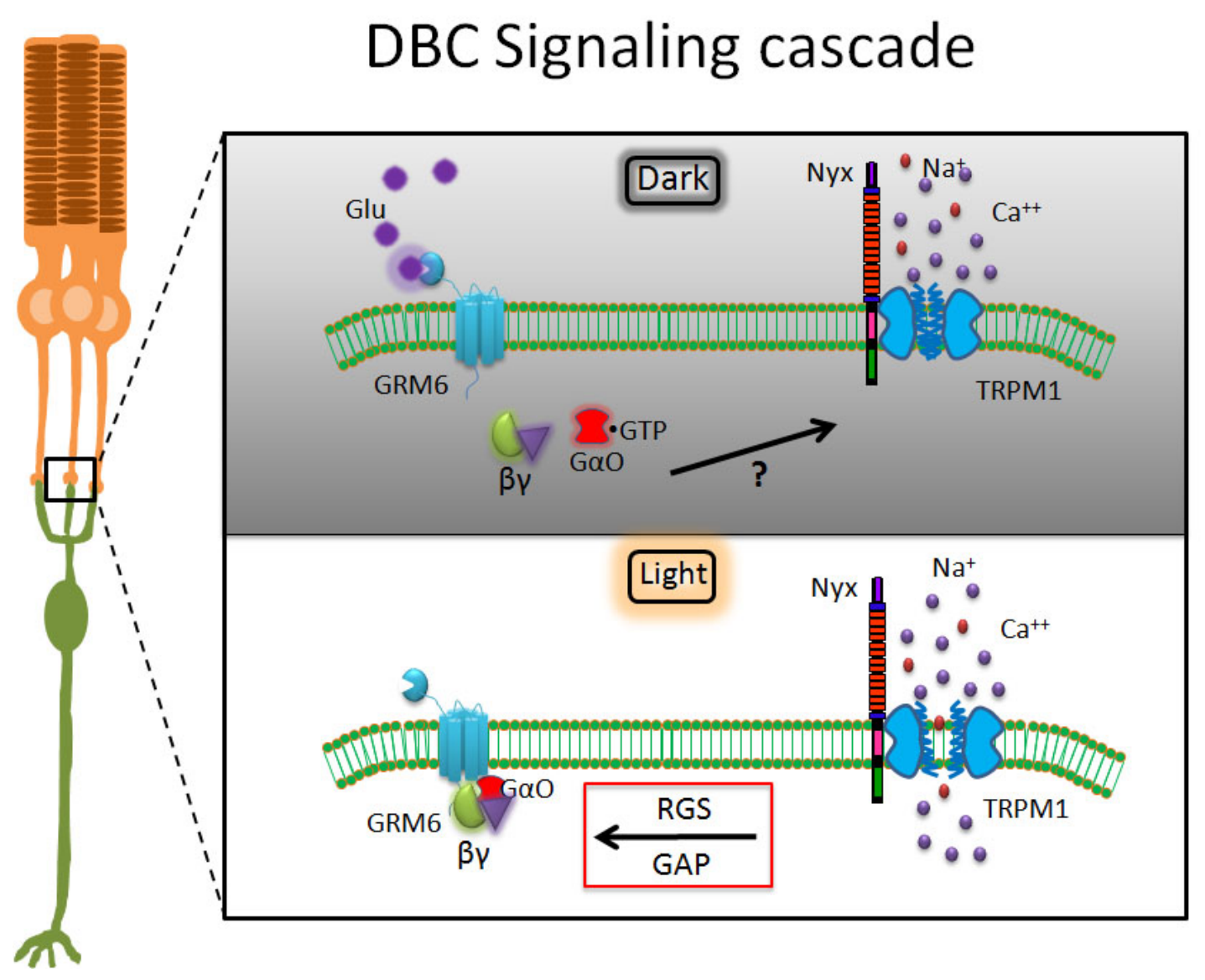

Figure 5: Schematic of depolarizing bipolar cell signaling. In the dark glutamate is tonically released into the synaptic cleft where it was bound by mGluR6, activating a $G$ protein cascade that results in the closure of the TRPM1 channel. In response to a light increment glutmate release into the synapse is decreased. The mGluR6 receptors are inactive because it is not bound to glutamate and the RGS proteins deactivate any remaining active $G$ proteins and the TRPM1 channel opens, causing the cell to depolarize. 


\section{Photoreceptor Light Sensitivity}

The retina is capable of operating over a range in illuminance of a factor of $10^{11}$ (Burkhardt and Gottesman, 1987; Burkhardt, 1994; Stockman and Sharpe, 2006). To achieve this, the retina uses two different types of photoreceptors with differing sensitivities to light (Fig. 6). The rod photoreceptors are the most sensitive and function under scotopic lighting conditions (luminance $10^{-2}$ to $\left.10^{-6} \mathrm{~cd} / \mathrm{m}^{2}\right)$ (Hood et al., 1979). A cloudy night sky without moon is equivalent to $\sim 10^{-4} \mathrm{~cd} / \mathrm{m}^{2}$ and a clear night sky with full moon is equivalent to $\sim 10^{-2} \mathrm{~cd} / \mathrm{m}^{2}$ (Middleton and Mayo, 1952). In humans, a light stimulus of only a few photons is capable of creating a visual percept (Hecht et al., 1942; Van Der Velden, 1946; Sakitt, 1972). Individual rods are capable of reliably responding to single photon stimuli and relaying this signal to retinal interneurons (Hecht et al., 1942; Field et al., 2005). Due to the rods only being active under scotopic conditions there is no color vision because rods only have one photopigment. Visual acuity of rodmediated vision is poor because of the high degree of signal convergence.

At the upper limit of rod sensitivity and lower limit of cone sensitivity is mesopic lighting conditions, with luminance from $10^{-2}$ to $1 \mathrm{~cd} / \mathrm{m}^{2}$ (Hood et al., 1979). Mesopic conditions are equivalent to a clear night sky with a full moon to a clear sky just after sunset (Middleton and Mayo, 1952). Under mesopic conditions color vision is poor, but visual acuity is improved due to the use of the less convergent cone visual pathway. Under photopic lighting $\left(1\right.$ to $\left.10^{6} \mathrm{~cd} / \mathrm{m}^{2}\right)$ conditions only the cone photoreceptors are functional (Jones et al., 1993; Burkhardt, 1994). The photopic visual range is equivalent to just after sunset on a clear day to a bright sunny day. Color vision and high visual acuity are possible because vision is primarily foveal (Middleton and Mayo, 1952; Hood et al., 1979). 


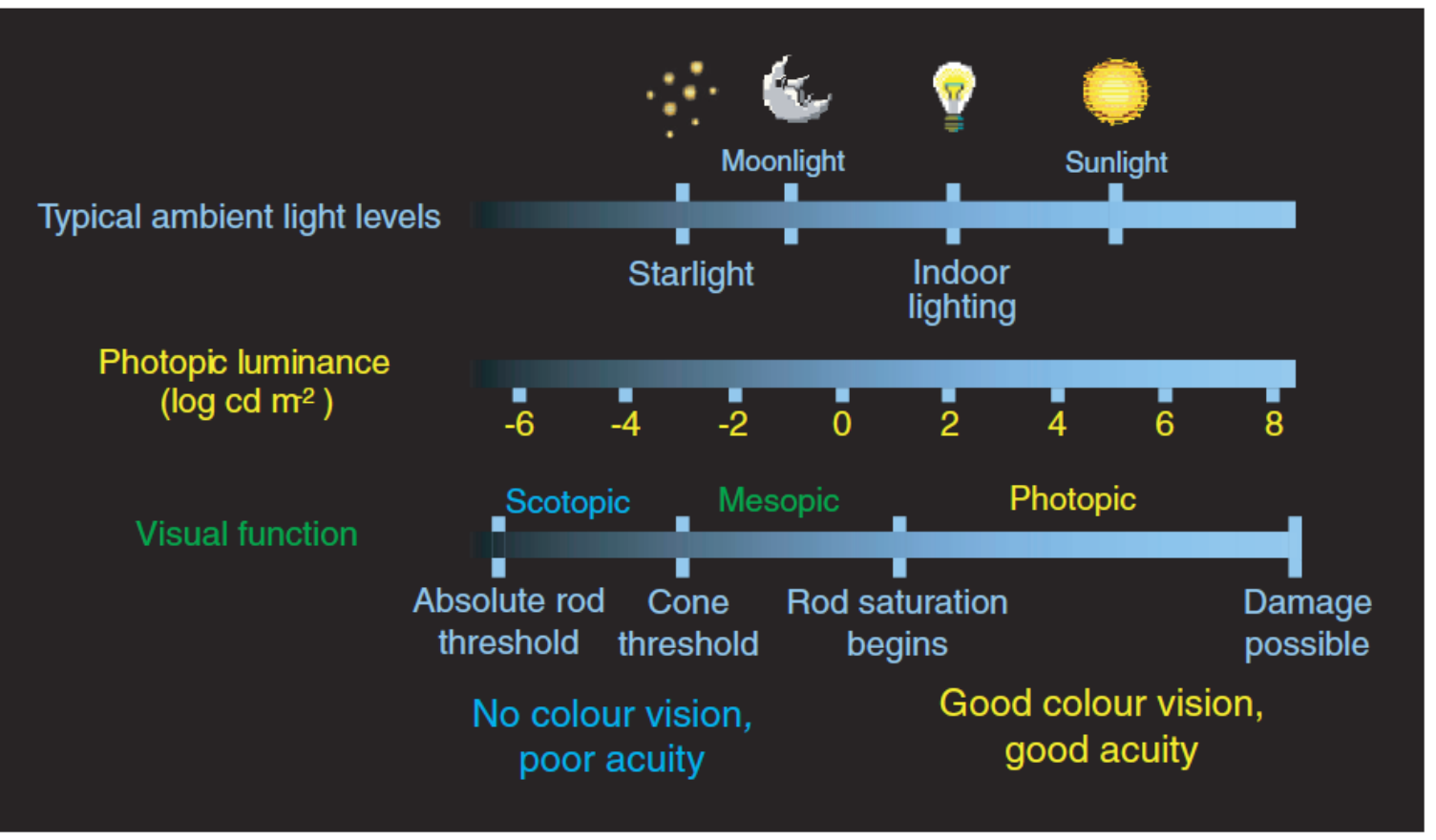

Figure 6: Photoreceptor Illumination Sensitivity. The diagram correlates typical ambient light levels to photoreceptor sensitivities. Modified from (Stockman and Sharpe, 2006) 


\section{Electroretinogram}

The activity of retinal neurons can be assessed non-invasively by the electroretinogram (ERG). The ERG measures the gross potential of the corneal surface in response to a light stimulus and produces waveforms that reflect electrical activity of specific neural layers. In 1933, Ragnar Granit reported that the ERG is composed of three components that last the entire duration of the light stimulus and can be isolated by deepening the state of anesthesia of the test animal (Fig. 7 and Granit (1933)). Granit won the Nobel Prize in Physiology and Medicine in 1967 for his work on the ERG. The first waveform produced by the ERG is the negative deflecting a-wave that reflects the hyperpolarization of the photoreceptors in response to an increase in luminance (Brown and Murakami, 1964; Brown, 1969; Penn and Hagins, 1969). The second wave produced by the ERG is the corneal positive b-wave that is produced by the depolarization of the bipolar cells (Slaughter and Miller, 1981; Stockton and Slaughter, 1989; Gurevich and Slaughter, 1993). Only the ON BCs contribute to the b-wave of the ERG. The OFF bipolar cells contribute to the repolarization of the ERG after the b-wave peak at light offset (Bush and Sieving, 1994). The c-wave is a slow forming positive wave that originates from RPE and glial cells (Noell, 1954; Steinberg et al., 1970).

The ERG can be measured under different lighting conditions to isolate specific neural pathways in the retina. The scotopic ERG is used to measure rod responses. By dark adapting the retina and using flash responses below cone threshold the rods and rod $B C$ responses can be isolated. The photopic ERG is used to measure cone and cone $B C$ responses. To measure the photopic ERG the test is performed in the presence of an adapting background that saturates the rods.

The ERG has been an invaluable tool for assessing retinal function and identifying defects in humans and mice (Peachey and Ball, 2003). A reduction in the awave of the ERG is indicative of defects in phototransduction or a reduction in the 
number of photoreceptor outer segment disks. A reduction in the b-wave of the ERG may indicate a disruption in neurotransmission from the photoreceptors to the bipolar cells (Peachey and Ball, 2003). This disruption may be caused by a defect on the pre or post-synaptic side of the synapse. The absence of the b-wave in the ERG has been associated with the non-progressive disease congenital stationary night blindness (CSNB). It has been reported that between 2.9 and $4.8 \%$ of the human population has a negative ERG and the underlying cause of the vast majority of negative ERGs is CSNB (Koh et al., 2001; Renner et al., 2006; Kim et al., 2012). 


\section{Electroretinogram}

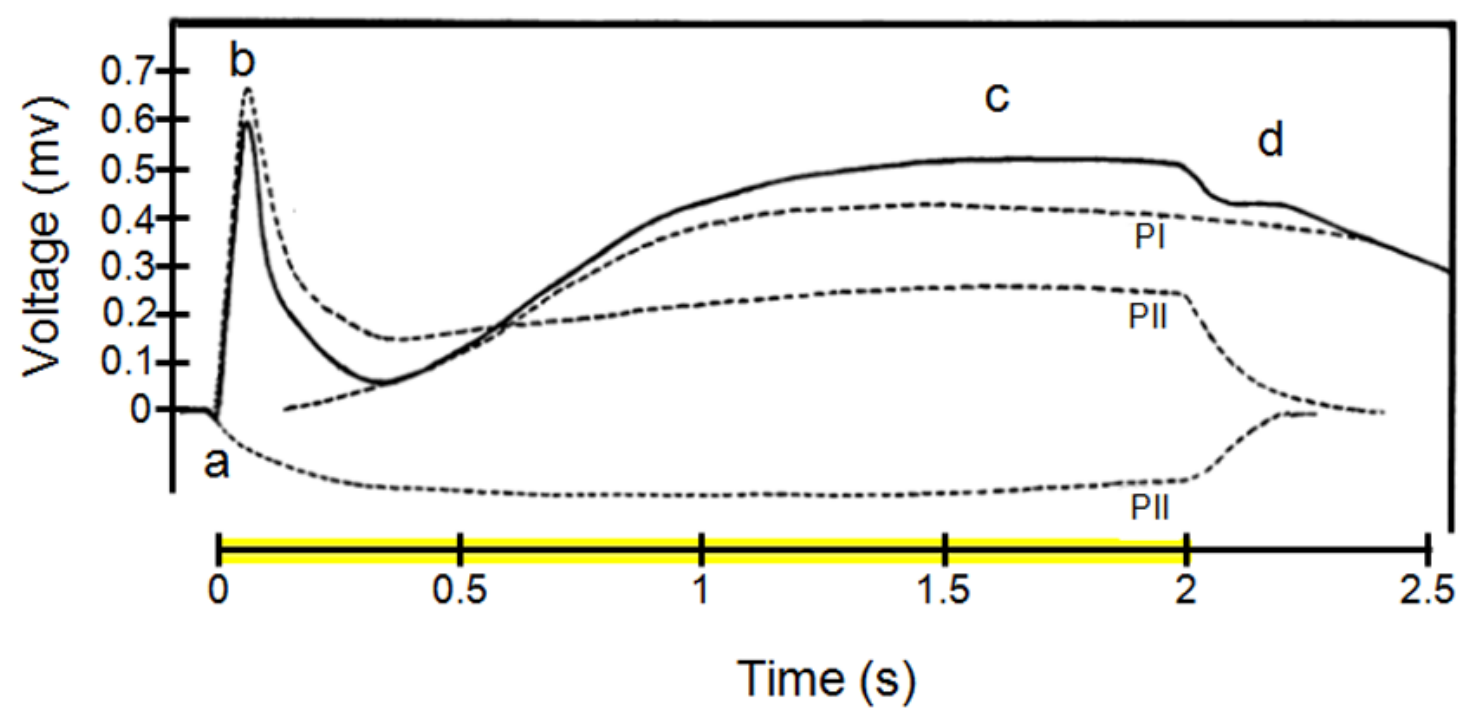

Figure 7: Components and waveforms of the Electroretinogram. The ERG response to a $2 \mathrm{sec}$ stimulus (yellow). The ERG waveforms (solid line) are labeled $\mathrm{a}, \mathrm{b}, \mathrm{c}, \mathrm{d}$ and the individual components (broken lines) are labeled PI, PII and PIII. Modified from (Granit, 1933) 


\section{Congenital Stationary Night Blindness}

Congenital stationary night blindness (CSNB) is a non-progressive disease that results in the disruption of rod signal transmission through the inner retina. Human patients with CSNB often have reduced visual acuity and reduced visual function under low light conditions (Miyake et al., 1986; Miyake et al., 1987; Audo et al., 2008). CSNB in humans is classified into two groups. Mutations that result in a complete ablation of the ERG b-wave are said to cause the complete form of CSNB (cCSNB) (Riggs, 1954; Miyake et al., 1986). A number of genetic mutations have been identified in genes that cause cCSNB. They include; Nyx (Pardue et al., 1998; Bech-Hansen et al., 2000; Pusch et al., 2000; Gregg et al., 2007), Grm6 (Masu et al., 1995; Dryja et al., 2005; Zeitz et al., 2005; Pinto et al., 2007), Trpm1 (Audo et al., 2009; Morgans et al., 2009; Koike et al., 2010b; Nakamura et al., 2010), Gpr179 (Audo et al., 2012; Peachey et al., 2012b) and Lrit3 (Zeitz et al., 2013b).

The incomplete form of CSNB (iCSNB) is characterized by a reduced b-wave as measured by ERG (Miyake et al., 1987). iCSNB is the result of disruptions in neurotransmitter release from the photoreceptor terminals and genes involved include Cacna1f (Strom et al., 1998), Cacnb2 (Ball et al., 2002; McCall and Gregg, 2008) and Cabp4 (Zeitz et al., 2006).

A study by Bijveld et al., (2013) characterized the visual defects of people with cCSNB and iCSNB. cCSNB patients report difficulty in performing tasks at night such driving, cycling, and maneuvering in dimly lit social settings such as movie theaters and pubs (Bijveld et al., 2013a). Many cCSNB patients report being blind at night and dependent on others in dark circumstances (Bijveld et al., 2013a). iCSNB patients report to having difficulty at the aforementioned tasks at a reduced rate and none felt they were always blind at night and dependent on others (Bijveld et al., 2013a). 
The screening of mice with the ERG has been critical to identifying spontaneous mutant mice with DBC dysfunction. Mapping and cloning the causative mutations has led to the identification of novel genes critical to the glutamate signaling cascade in DBCs (Pardue et al., 1998; Gregg et al., 2007; Peachey et al., 2012b). The screening of human patients with previously unidentified causes of CSNB for the orthologous mouse gene has identified genes that cause CSNB (Bech-Hansen et al., 2000; Pusch et al., 2000; Audo et al., 2009; Nakamura et al., 2010; Audo et al., 2012; Peachey et al., 2012b).

cCSNB mouse models known to date have a normal retinal morphology as visualized at the light or electron microscope level (Masu et al., 1995; Pardue et al., 1998; Tagawa et al., 1999; Dhingra et al., 2000; Pardue et al., 2001; Morgans et al., 2009; Koike et al., 2010b). iCSNB mouse models have abnormal retinal morphology highlighted by ectopic bipolar and horizontal cell dendrites that extend into the ONL (Ball and Gregg, 2002; Dick et al., 2003; Chang et al., 2006; Bayley and Morgans, 2007).

\section{G Protein-Couple Receptor Signaling}

GPCRs are critical for phototransduction and signaling through the inner retina. The retina is a very attractive system for studying GPCR signaling mechanisms because of the availability of mutant mice, accessibility and ability to functionally assess specific signaling events. GPCRs are the target of approximately $30 \%$ of pharmaceutical drugs (Salon et al., 2011). The more knowledge gained about these diverse signaling pathways the better we are able to develop effective therapies for diseases that impair vision. All GPCRs are membrane proteins that contain an extracellular amino terminus followed by a 7 transmembrane (TM) domain and a cytosolic carboxy terminus. GPCRs represent the largest class of signaling proteins in the human genome with more than 800 members (Fredriksson et al., 2003). 
GPCRs have been classified into three main families based on shared homology. Class $A$ are the rhodopsin like receptors, Class B are the secretin-like receptors and Class C are the metabotropic glutamate-like receptors (Foord et al., 2005). The simplistic model of GPCR signaling is that binding of an extracellular ligand produces a conformational change in the receptor that activates heterotrimeric $\mathrm{G}$ proteins (Gaßy) inside the cell. The ligands for GPCRs are a diverse group including small compounds, ions, peptides and amino acids (Muller et al., 2008). The majority of GPCRs initiate intracellular signaling through heterotrimeric $G$ proteins but it is beginning to be understood that some operate through $\mathrm{G}$ protein-independent mechanisms (Galandrin et al., 2007; Altier and Zamponi, 2008).

The extracellular domain of GPCRs consists of the amino terminus and the extracellular loops (ECLs) ECL1, ECL2 and ECL3. There is large diversity in sequence length and composition among the receptors amino terminus (Lagerstrom and Schioth, 2008). Class A GPCRs form a ligand-binding pocket with the ECLs that can either be occluded or water-accessible (Palczewski et al., 2000; Hanson et al., 2012). Class B GPCRs have an amino terminus ligand binding domain composed of an $\alpha$-helix and two $\beta$-sheets stabilized by disulfide bridges (Parthier et al., 2009). Class C GPCRs typically possess a large bilobed amino terminal ligand-binding domain coined the venus flytrap (VFT) domain (Chun et al., 2012). The VFT ligand binding domain shares sequence homology with bacterial periplasmic binding proteins (PBP) (Zhang et al., 2008). An accepted hypothesis for the origin of Class C GPCRs is that they are the result of a fusion between a rhodopsin-like receptor and a PBP (Pin et al., 2003).

The 7TM helix region of GPCRs serves to relay extracellular information to the inside of the cell (Muller et al., 2008; Venkatakrishnan et al., 2013). The crystallization of GPCRs bound to agonist and ligand free GPCRs provided detail about the 7TM movement that occurs when the receptor is activated (Audet and Bouvier, 2012). 
Movement of the 7TM region cause a conformational change that allows the binding of heterotrimeric G proteins by the intracellular regions (IC) of the GPCR (Audet and Bouvier, 2012).

The IC loops of GPCRs typically bind downstream signaling effectors such as G proteins, kinases and arrestins (Rasmussen et al., 2011; Katritch et al., 2012). The IC regions undergo dramatic conformational changes in response to ligand binding (Park et al., 2008; Scheerer et al., 2008; Choe et al., 2011; Lebon et al., 2011; Rasmussen et al., 2011; Standfuss et al., 2011; Xu et al., 2011). The conformational change allows the binding of the receptor's associated GDP bound heterotrimeric G proteins (Latek et al., 2012). The conformation of the GDP Gaßy when bound to the GPCR facilitates GTP for GDP nucleotide exchange resulting in release of the activated GaGTP and dissociation from the Gßy complex (Chung et al., 2011; Rasmussen et al., 2011).

Some GPCRs are capable of functioning as monomeric receptors while others require assembly into dimers or larger complexes (Bouvier, 2001; Chabre and le Maire, 2005; Milligan, 2006; Bayburt et al., 2007; Whorton et al., 2007; Rondard et al., 2008; Rondard et al., 2011; Yanagawa et al., 2011). In addition to forming complexes with other GPCRs, they may also associate with other transmembrane proteins to form signaling complexes (Altier and Zamponi, 2008; Altier, 2012). This is believed to enhance signaling kinetics.

The rhodopsin GPCR signaling pathway is the prototypical GPCR pathway. The first crystal structure of a GPCR was of bovine rhodopsin (Palczewski et al., 2000), since then the crystal structure of 18 different class A GPCRs has been solved (Venkatakrishnan et al., 2013). As a result, much of what we know about GPCR signaling pertains to class A GPCRs and a one-size-fits all approach has typically been used when studying GPCRs from other classes. While this approach has proven useful, it has become clear with the growing diversity of GPCRs that not all will fit that mold. It 
is important to identify these novel mechanisms of GPCR signaling to not only enhance our understanding of GPCR signaling but to unlock answers that may prove therapeutically beneficial.

\section{What we can learn from the retina}

The retina is a model system that allows us to learn about more than just vision. It is the most accessible part of the CNS allowing us to more easily study the development of complex neural circuitry. The retinal neurons offer a glimpse of neural signal processing that we understand must also take place in the brain, but are not as easily measured. The retina can be easily genetically manipulated and the results of these manipulations can be functionally assessed with visual tests, allowing perhaps a more accurate depiction of gene function in neural circuits than in other regions of the CNS. 


\section{CHAPTER II: GPR179 IS MUTATED IN THE NOB5 MOUSE}

Aim 1: Identify the mutation responsible for the nob phenotype in the nob5 mouse Rationale: Spontaneous mutant mice are important tools for identifying genes involved in disease. The nob5 mouse lacks the ERG b-wave and is predicted to have a defect in the glutamate-signaling cascade of DBCs. By identifying the mutation in the nob5 mouse we can better understand how the glutamate receptor, mGluR6, gates the TRPM1 cation channel closed in the DBCs. As a testament to our incomplete knowledge of the glutamate signaling cascade in the DBCs, there are patients with cCSNB that do not have mutations in the known cCSNB causing genes (Nakamura et al., 2010; Bijveld et al., 2013b). Therefore, it is probable that there are cCSNB patients with a mutation in the nob5 ortholog. Although there currently is no cure for cCSNB and patients have relatively minor lifestyle inconveniences (Bijveld et al., 2013a), identifying the cause of their disease can provide closure and the knowledge that they do not have a progressive retinal disease in addition to the scientific value of understanding a novel GPCR pathway.

Hypothesis: The nob5 mouse has a mutation in a gene expressed in the ON BCs (DBCs) of the retina.

\section{Introduction}

The ERG is a common tool used to evaluate visual function in mice and can be used as a high-throughput method for identifying retinal dysfunction (Dalke et al., 2004). The screening of mice using the ERG has identified spontaneous mouse mutants with DBC dysfunction (Maddox et al., 2008; Peachey et al., 2012a; Peachey et al., 2012b). 
In some of these cases, the mutated genes were novel (Bech-Hansen et al., 2000; Peachey et al., 2012b) and required various molecular biology techniques to locate the mutation within the genome. In other cases, the mutations were in genes already known to cause the nob phenotype but offer unique insights into protein properties that the knockout model does not display (Maddox et al., 2008; Peachey et al., 2012a).

The nob5 mouse is a spontaneous mutant that arose on a $\mathrm{C} 3 \mathrm{H} / \mathrm{HeJ}$ mouse strain. The $\mathrm{C} 3 \mathrm{H} / \mathrm{HeJ}$ mouse strain carries a homozygous mutation in the rod phosphodiesterase gene, $P d e 6 b$, and was an early model for retinal degeneration (Sidman and Green, 1965; Pittler and Baehr, 1991). This form of retinal degeneration progresses quickly and causes blindness by weaning age, because of a complete loss of photoreceptors (Farber and Lolley, 1974; Lolley and Farber, 1976). The retinal degeneration in the $\mathrm{C} 3 \mathrm{H} / \mathrm{HeJ}$ mouse strain allowed the nob5 phenotype to go unnoticed until the $\mathrm{C} 3 \mathrm{H}$ line was crossed to a congenic $\mathrm{C} 3 \mathrm{H}$ line lacking the $P d e 6 b$ mutation. It was then that an ERG screen by Gianluca Tosini at Morehouse College of Medicine discovered the nob5 mutant.

The nob5 mouse has a normal a-wave but lacks a b-wave in the scotopic ERG (Fig. 8A). The scotopic ERG measures responses through the rod photoreceptor and rod BC pathway. These data indicate that the rod photoreceptors are functioning normally, but the rod BCs are not. The nob5 mouse has a normal a-wave in the photopic ERG but lacks a b-wave (Fig 8B). The light adapted or photopic ERG measures responses mediated by cones. These data indicate the cone photoreceptor phototransduction is normal but cone DBC signaling is completely absent. The ERG of the nob5 mouse (Fig. 8A,B) is similar to other cCSNB mouse models (Masu et al., 1995; Pardue et al., 1998; Pinto et al., 2007; Maddox et al., 2008; Morgans et al., 2009), which lead us to the hypothesis that the causative mutation would be in a gene expressed in the DBCs and critical for the function of the mGluR6 to TRPM1 cascade. 
A

B
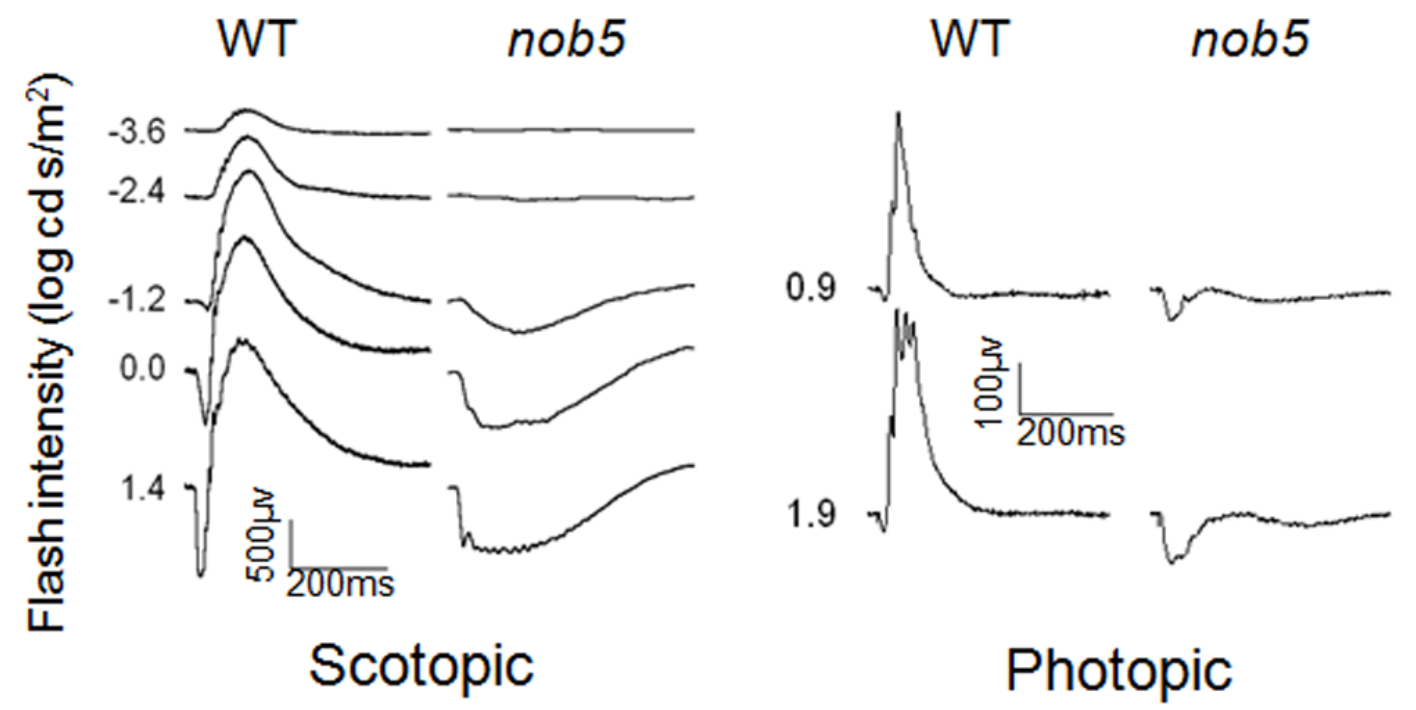

Figure 8: Scotopic and photopic ERGs of WT and nob5 mice. (A) Scotopic ERGs of WT and nob5 mice show nob5 mice have a normal ERG a-wave in response to increasing flash intensity but are lacking a b-wave. (B) Photopic ERGs show the nob5 mice lack a cone mediated $b$-wave response. Taken together these data indicate that there is likely a defect in DBC signaling in the nob5 mice. 
The phenotype in the nob5 mice displays an autosomal recessive inheritance pattern. To determine if the underlying mutation was in a gene known to cause DBC dysfunction, complementation crosses were performed at the Cleveland Clinic with established models of DBC dysfunction $\mathrm{Grm}^{n o b 3}, \mathrm{Nyx}^{\text {nob }}$ and Trpm $1^{-/}$. The F1 offspring were assessed for retinal function using the ERG. All offspring had normal ERGs indicating the mutant gene in nob5 mice was not Grm6, Nyx or Trpm1.

To localize the nob5 mutation within the genome a mapping cross was set up at the Cleveland Clinic. The nob5 mice were outcrossed to C57BL6/J mice producing nob5 ${ }^{-/+} \mathrm{F} 1$ offspring. The $\mathrm{F} 1$ offspring were subsequently intercrossed to produce $\mathrm{F} 2$ offspring with new allele combinations. The F2 offspring were phenotyped by ERG. DNA was pooled from 20 mice displaying the nob5 phenotype and genotyped at approximately 103 simple sequence repeat (SSR) polymorphic markers across the genome (Taylor et al., 1994) Genomic regions that were heterozygous for both mouse strains $(\mathrm{C} 3 \mathrm{H} / \mathrm{HeJ}$ and $\mathrm{C} 57 \mathrm{BL} 6 / \mathrm{J})$ were ruled out as containing the nob5 gene. This strategy mapped the nob5 allele to a 4.1 megabase (MB) region (Chr11:9456343898683655) on chromosome 11.

\section{Results}

\section{Linkage mapping reduced the nob5 critical region to $1.3 \mathrm{Mb}$}

The SSR low-resolution genome mapping localized the nob5 phenotype between MIT Markers D11MIT288 (Chr11:94563438) and D11MIT14 (Chr11:98683655) on chromosome 11. The region between D11MIT288 and D11MIT14 spans $4.1 \mathrm{Mb}$ and is equivalent to 3.5 centimorgans (cM) (http://cgd.jax.org/mousemapconverter/). To reduce the region containing the nob5 mutation, which was thought to arise on a $\mathrm{C} 3 \mathrm{H}$ background, crosses were set up in which the nob5 mouse was outcrossed with C57BL6/J mice. These two strains can be easily differentiated with various SSR and single nucleotide polymorphism (SNP) markers, which allows for the detection of 
chromosomal crossovers to narrow the critical region. The SSR and SNP genotyping markers were identified using the National Center for Biotechnology Information's dbSNP database and confirmed by Sanger sequencing. SNPs were genotyped using a high throughput method referred to as high resolution melting (HRM), which relies on melting curve analyses on an ABI $7900 \mathrm{HT}$ real-time PCR machine. SSR markers were genotyped by running amplified samples on agarose gels. Because the nob5 critical region is small we expect only $5 \%$ of mice to have a crossover in the critical region. Therefore we used a two-step genotyping and phenotyping strategy.

In total $1000 \mathrm{~F} 2$ mice were generated from a backcross of F1 (nob5 $\mathrm{x}$ C57BL/6J) mice to nob5 mice. All 1000 mice were genotyped with D11MIT14 and D11MIT288 markers to identify animals with chromosomal recombination events within the nob5 critical region. These animals with chromosomal crossovers then were genotyped using a combination of nine different MIT and SNP markers spanning the critical region to further localize the recombination event (Fig. 9B). ERGs were performed on the mice with crossovers that should narrow the critical region.

Of the $\sim 1,000$ backcross progeny, 39 had crossovers between the D11MIT288 and D11MIT14 markers, reducing the nob5 critical region to $\sim 1.3 \mathrm{MB}$. The $\sim 1.3 \mathrm{MB}$ region was situated in a highly gene rich region on chromosome 11 (Zody et al., 2006) containing $\sim 80$ predicted genes (Fig. 9B). Due to the fact that 1000 mice were needed to reduce the region to a $1.3 \mathrm{MB}$ region of high gene density, it became clear further refinement using this genetic approach was not feasible. Therefore two strategies were used to identify the causative mutation in the nob5 mouse, a candidate gene search and next-generation sequencing of the region. 


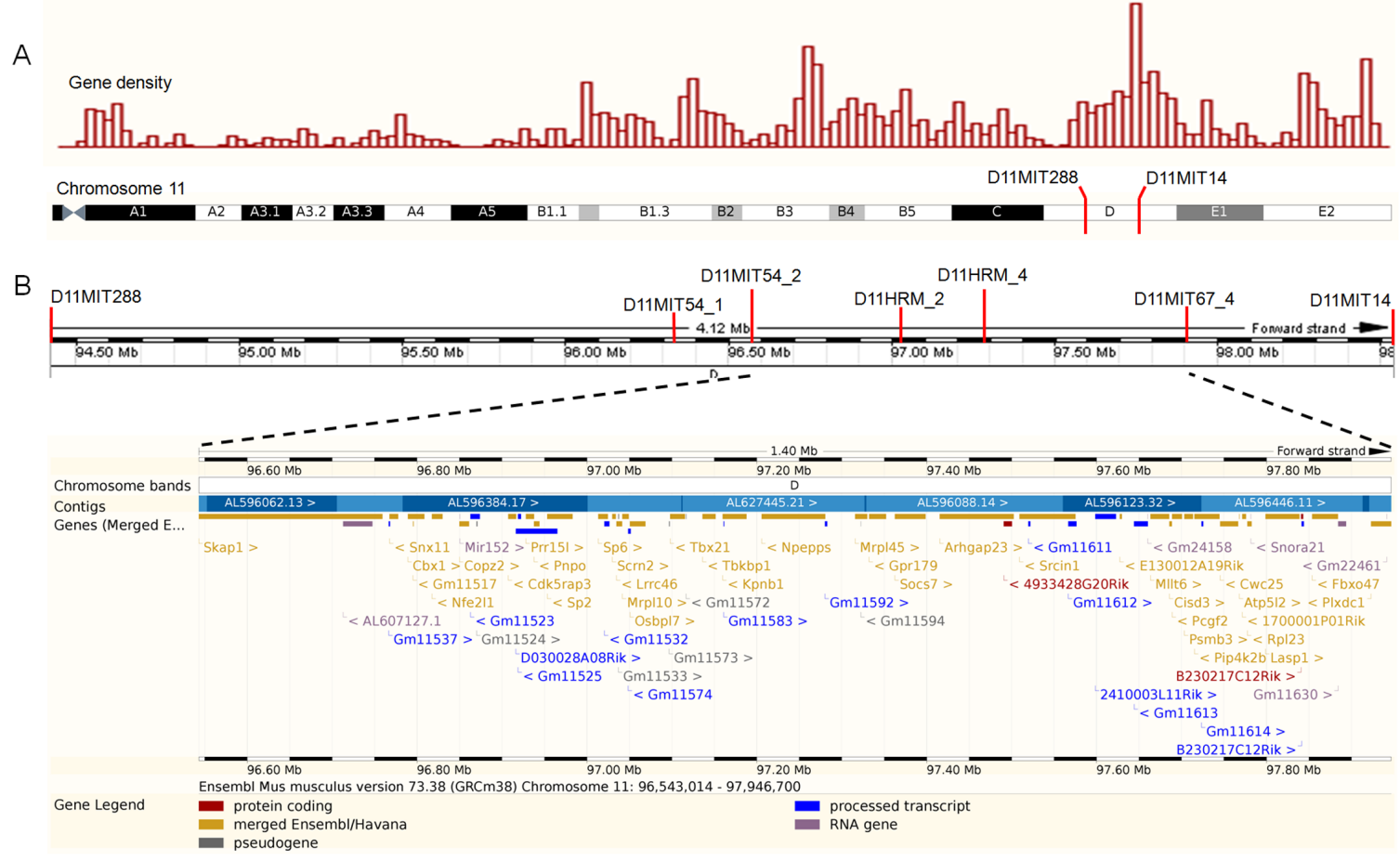

Figure 9: Mapping panel of nob5 critical region. (A) Chromosome map of mouse chromosome 11 with the protein coding gene density (top) mapped to the chromosome. (B) Chromosome map of nob5 critical region including genotyping markers used to narrow the region. (Bottom) The $1.3 \mathrm{Mb}$ region between markers D11MIT54_2 and D11MIT67_4 contained $\sim 80$ predicted genes. 
Genes located within the critical region were evaluated for possible roles in DBC signaling. Genes that had known roles in signaling, such as ion channels or had expression in neuronal systems were considered candidates. Genes for which knockout mice had already been made and showed a lethal phenotype were excluded as candidates because the nob5 mouse is otherwise healthy.

To identify mutations in candidate genes RNA was isolated from nob5 mouse retinas and converted into cDNA. Primers were made that flanked the open reading frame (ORF) and were used to amplify the ORF and clone it into a pCR4-TOPO vector (Invitrogen). The ORFs were then sequenced and compared to the reference genome. Genes thought to be good candidates included Npepps, Arhgap23, Pip4k2b, Srcin1 and Cacnb1, however, none had mutations in the ORFs.

\section{Next generation sequencing identified an insertion in Gpr179}

Therefore, to identify the mutant gene we decided to sequence the entire 1.3 MB region using next generation sequencing. This method requires capturing and amplifying genomic DNA from the critical region using microarray sequence capture (Albert et al., 2007). The sequence capture array was designed and made off site by Roche NimbleGen and was designed to capture a $1.5 \mathrm{MB}$ region of DNA that included the entire nob5 critical region plus upstream and downstream flanking regions, but excluded highly repetitive regions. I used the UCSC genome browser to ensure the probes on the array design would capture all of the gene containing regions within the nob5 critical region.

Sequencing of the captured DNA was performed on an Illumina GAllx sequencing machine by Ambry Genetics. Mouse liver genomic DNA was isolated from a nob5 mouse and a $\mathrm{C} 3 \mathrm{H}$ control and shipped to Ambry Genetics for enrichment of the target region and sequencing using 54bp paired-end sequencing. The bioinformatics report was prepared by Ambry Genetics and delivered to the lab, along with a hard drive 
containing the raw FASTQ sequence files. The bioinformatics report contained two points of interest. There were no unique non-synonymous point mutations found in the nob5 sample compared to the control and there was a possible transposable element insertion in the nob5 sample that was not found in the control sample. The sequence files identified two $\sim 30 \mathrm{bp}$ of junction fragments on the 5 prime and 3 prime ends of a possible insertion site at Chr11:97212072-97212086, which is within intron 1 of the Gpr179 gene.

To confirm the insertion and determine its size, primers were created that flanked the putative insertion and PCR was used to amplify the DNA fragment in the nob5 and control samples. The PCR results showed that DNA from the nob5 mouse contained an insertion that is approximately $6.5 \mathrm{~kb}$ (Fig. 10A,B). Sequence analyses of the PCR fragment indicated it was a retrotransposable element of the ERV2 class, although complete sequencing was not possible because of its repetitive nature. 


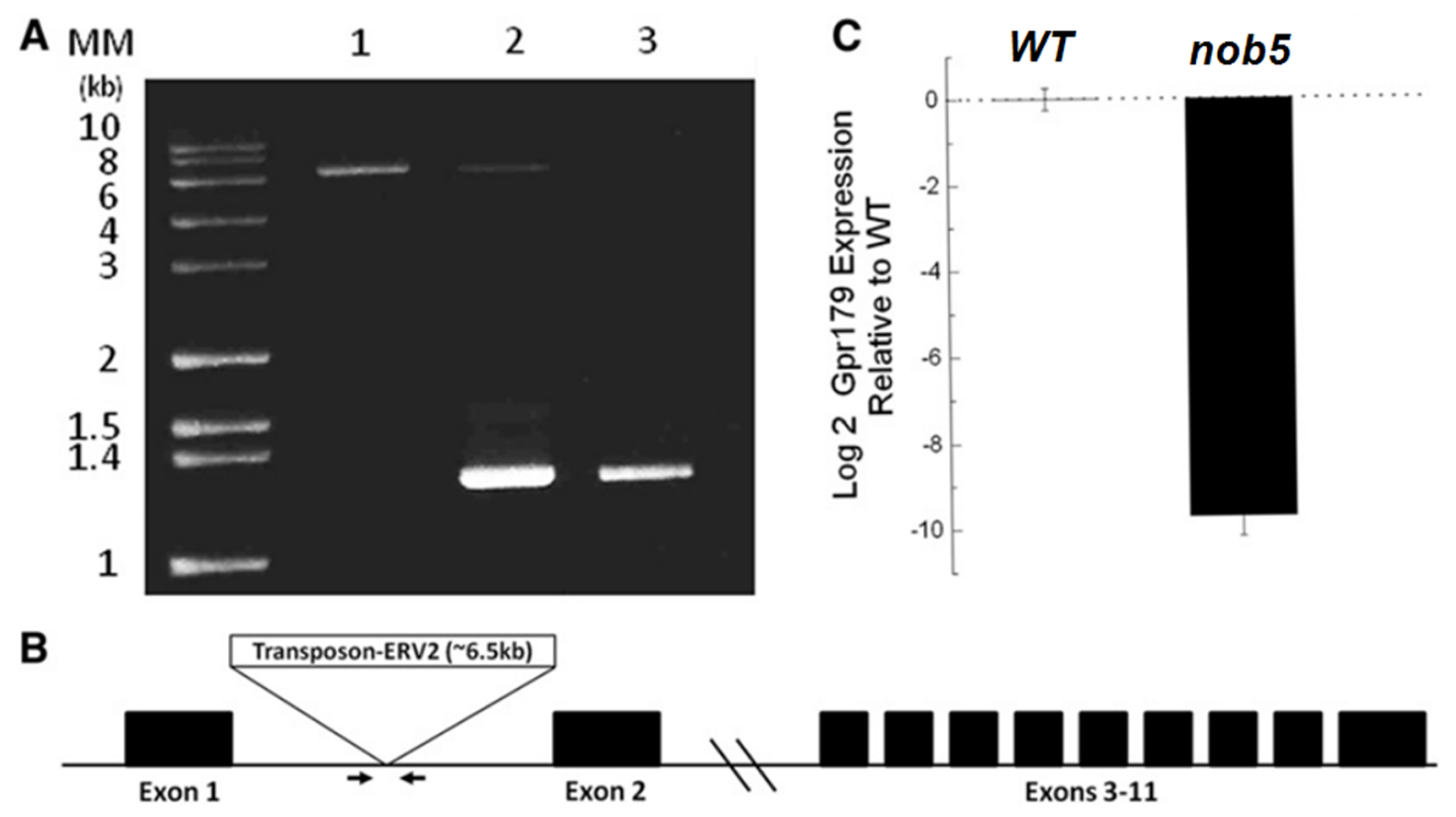

Figure 10: A transposable element disrupts Gpr179 expression. (A) PCR amplification of the insertion followed by agarose gel electrophoresis revealed the insertion was approximately $6.5 \mathrm{~kb}$ in length. (B) Schematic of Gpr179 exon map and insertion site. Subsequent sequencing of the insertion fragment revealed it was a transposable element of the ERV2 class. (C) Quantification of Gpr179 expression at the exon 1-2 boundary using a taqman assay. Gpr179 expression was normalized to 18S RNA and is relative to WT gene expression. Error bars indicate mean and standard deviation for three mice. Gpr179 expression is significantly reduced in the nob5 mouse $(p<0.0005)$. From Peachey et al., 2012b. 


\section{The transposon insertion dramatically decreases Gpr179 gene expression}

To understand how the transposable element affected Gpr179 gene expression in the nob5 sample two methods were used. First, using primers nested in exon 1 and exon $11, \mathrm{I}$ attempted to clone a cDNA fragment made from WT and nob5 retinal RNA. PCR amplification using cDNA from WT resulted in the expected fragment size of $\sim 6.8$ $\mathrm{kb}$, whereas the nob5 sample did not produce a PCR product. Forward and reverse primers were then created across the gene designed to amplify smaller fragments of the predicted cDNA to see if any of the Gpr179 mRNA was expressed and processed in nob5 retinas. Primers placed in exon 2 through exon 11 yielded PCR fragments of the expected size in the WT and nob5 sample. Forward primers placed in exon 1 and reverse primers placed in any exon downstream of the transposon insertion (exons 211), failed to produce a PCR fragment in the nob5 samples. These experiments confirm that Gpr179 is expressed in the retina and that gene expression is altered in the nob5 retina.

To quantitatively measure Gpr179 gene expression in the nob5 sample I used two different TaqMan Assays (Life Technologies). The first (Mm00615352_m1) spanned the exon1/2 boundary of Gpr179 and had an amplicon length of $83 \mathrm{bp}$. The second (Mm04204651_m1) spanned the exon 5/6 boundary and had an amplicon length of 103 bp. The results of the experiment show that gene expression is virtually undetectable in the nob5 mouse using the exon1/2 TaqMan assay (Fig. 10C). The exon5/6 TaqMan assay revealed that Gpr179 expression downstream of exon 1 showed only a slight increase compared to WT (data not shown).

These experiments indicate that exons downstream of intron 1 are expressed and processed but intron 1 is not processed properly. The transposable element insertion presumably interferes with processing of intron 1 because it likely contains many splice acceptor and splice donor sites that may result in a large number of different 
mRNAs. These splice variants would likely create transcripts that undergo nonsensemediated decay. Exons downstream of exon 1 appear to be processed correctly. It seems unlikely that the loss of exon 1 would cause a hypomorphic mutation because exon 1 contains the translation start site, the signal sequence and the majority of the protein's extracellular amino terminus. It would be unlikely that GPR179, a 7 TM spanning protein could have any function without these critical domains.

\section{In situ hybridization localizes Gpr179 mRNA to the INL of the retina}

My RT-PCR experiments had determined that Gpr179 is expressed in the retina, but it was not known by which cells. To localize Gpr179 gene expression I used in situ hybridization (ISH) on fixed retinal slices. Antisense digoxigenin (DIG) labeled RNA probes were created by cloning a $\sim 800$ bp cDNA fragment encoded by Gpr179 into a pSPT18 vector and performing in vitro transcription using T7 RNA polymerase. The ISH was performed on fixed retinal slices and visualized by probing for the DIG RNA with horseradish peroxidase (HRP) labeled antibodies followed by Cy3 tyramide signal amplification (TSA). Immunostaining with PKC antibodies, which labels the rod BCs, was performed to give a cellular reference to identify in which cells the RNA was expressed.

The Gpr179 antisense probes annealed in the INL of the retina where the bipolar cell bodies reside (Fig. $11 \mathrm{~A}$ ). The antisense probes did not anneal to any of the other nuclear layers. As a positive control, antisense probes were designed against Grm6 mRNA, the glutamate receptor expressed in the DBCs with a known ISH expression profile (Kim et al., 2008). The antisense Grm6 DIG probes labeled the INL of the retina as identified by co-labeling with PKC and gave a similar staining pattern as Gpr179 (Fig. $11 \mathrm{~A}, \mathrm{~B})$. These data suggest Gpr179 is expressed in the DBCs of the retina. 


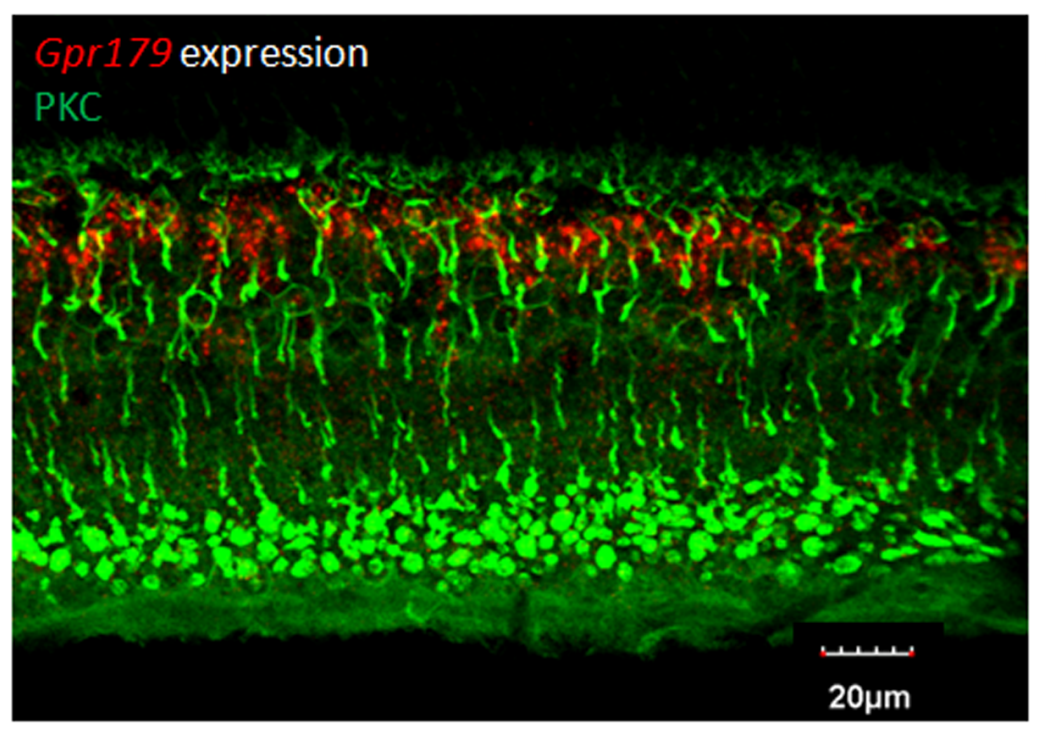

ONL

OPL

INL

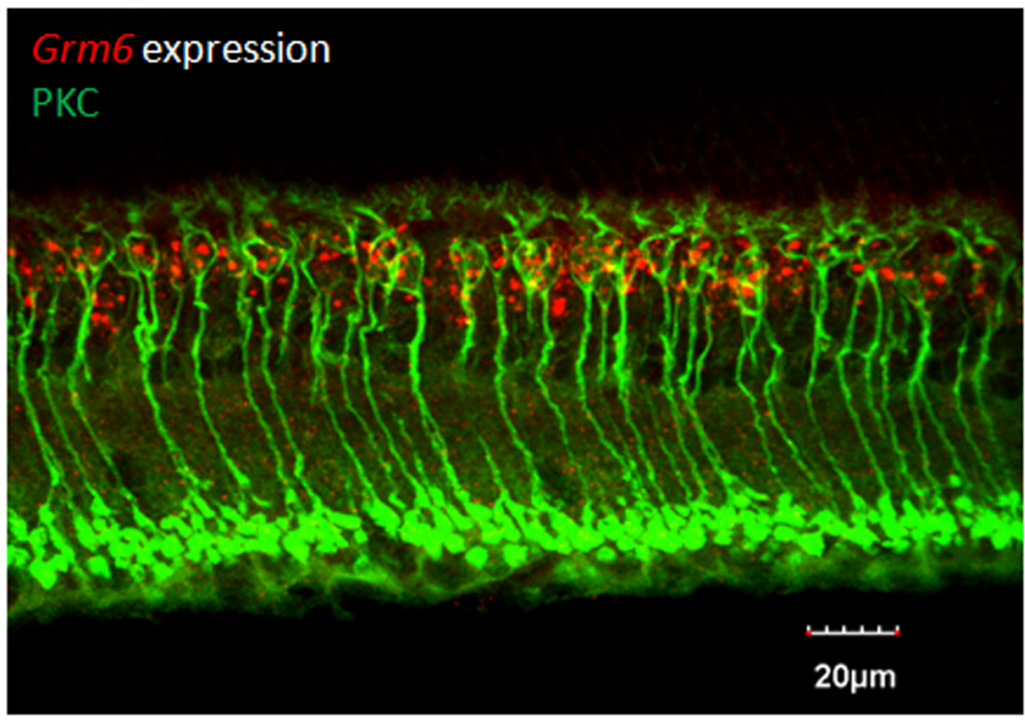

ONL

OPL

INL

IPL

Figure 11: GPR179 is expressed in bipolar cells. In situ hybridization localizes Gpr179 mRNA expression. (Top) Antisense Gpr179 probes (red) hybridize to the INL and expression overlaps with the rod BC bodies (green). Gpr179 expression mirrors the mRNA expression of Grm6 (bottom), a gene that is solely expressed in the DBCs of the retina. 


\section{GPR179 is expressed on the DBC dendritic tips in human retinas}

To localize GPR179 protein expression in the retina, immunostaining was performed using a commercially available antibody designed against a peptide (HPA017885 Sigma) that is located in the amino terminus of the extracellular domain of human GPR179. The human peptide shares $84 \%$ sequence identity with the orthologous mouse peptide. Immunostaining was performed on WT C57BL6/J retinal sections while co-labeling with peanut agglutinin (PNA), which binds to a lectin on cone pedicles in the OPL (Blanks et al., 1988; Haverkamp et al., 2001). The staining for GPR179 showed a staining pattern inconsistent with the known pattern of proteins expressed in the DBCs (not shown). Further, the pattern in WT and nob5 was indistinguishable suggesting the antibody was not recognizing mouse GPR179 (data not shown).

To determine if the antibody used above gave the predicted staining in human, immunostaining was performed on transverse human retinal sections. GPR179 staining in the human retinas showed a punctate staining pattern in the OPL typical of other proteins that are part of the DBC signal transduction cascade (Fig. 12). Because the antibody only stained puncta, I could not be determine if the staining was pre synaptic or post synaptic, but taken with the ISH data and ERG phenotype of human patients with mutations in GPR179, I predict it will be located post-synaptically. This was confirmed recently by immuno-electron microscopy of human retinas (Klooster et al., 2013). These data also indicate that the staining in the mouse retina by the human GPR179 antibody is non-specific. To study GPR179 protein expression in the mouse retina an antibody had to be developed that recognized the mouse protein. 


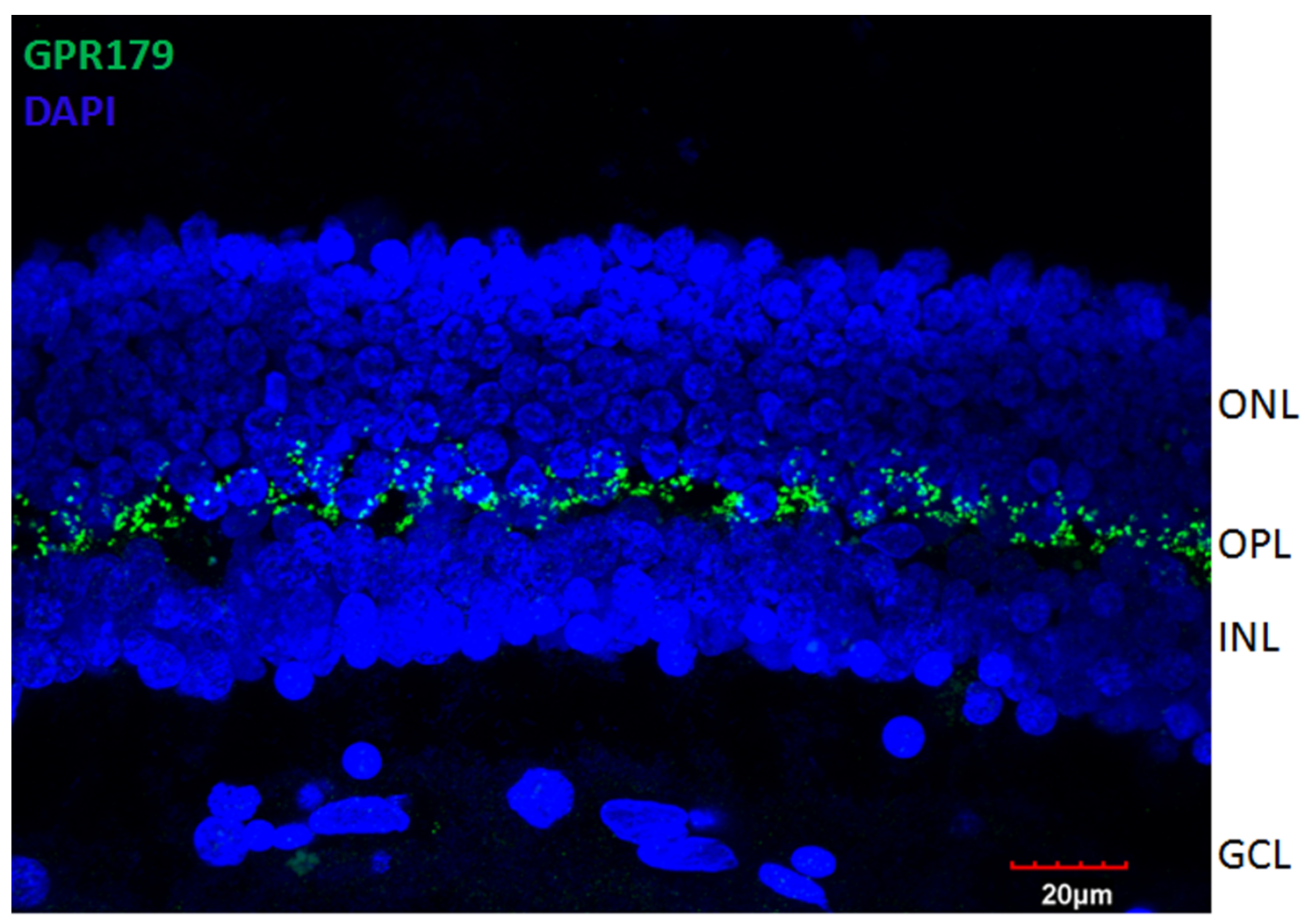

Figure 12: GPR179 is expressed in the OPL in human retina. GPR179 labeling (green) and DAPI (blue) in fixed transverse retinal sections from human retina gives a punctate staining pattern typical of proteins in the glutamate signaling cascade of the DBCs 


\section{GPR179 staining is absent in the OPL of the nob5 retina}

Due to the robust labeling of the human antibody in human retina, we used a portion of the orthologous mouse peptide sequence as the antigen (KVQEETPGEDLDRPVLQKR) for generation of our mouse antibody. Double labeling using antibodies against PKC, a rod bipolar cell marker, and the GPR179 antibody specific to mouse shows that in WT mouse transverse retinal sections GPR179 shows punctate staining at the tips of the rod bipolar cells in the OPL (Fig. 13), consistent with the pattern seen in the human retina (Fig. 12), and with other proteins expressed at the tips of the DBCs including TRPM1, GRM6 and NYX (Gregg et al., 2007; McCall and Gregg, 2008; Koike et al., 2010b; Orlandi et al., 2012; Peachey et al., 2012b; Zeitz et al., 2013b). Immunostaining of the nob5 retinas with PKC and the mouse GPR179 antibody revealed the absence of GPR179 expression in the OPL of the nob5 retina (Fig. 12) and (Peachey et al., 2012b). These data indicate that GPR179 is expressed in the DBCs and localizes to the DBC dendritic tips. The mutation in Gpr179 in the nob5 mouse disrupts GPR179 protein expression and further supports the hypothesis that GPR179 is required for DBC function. 


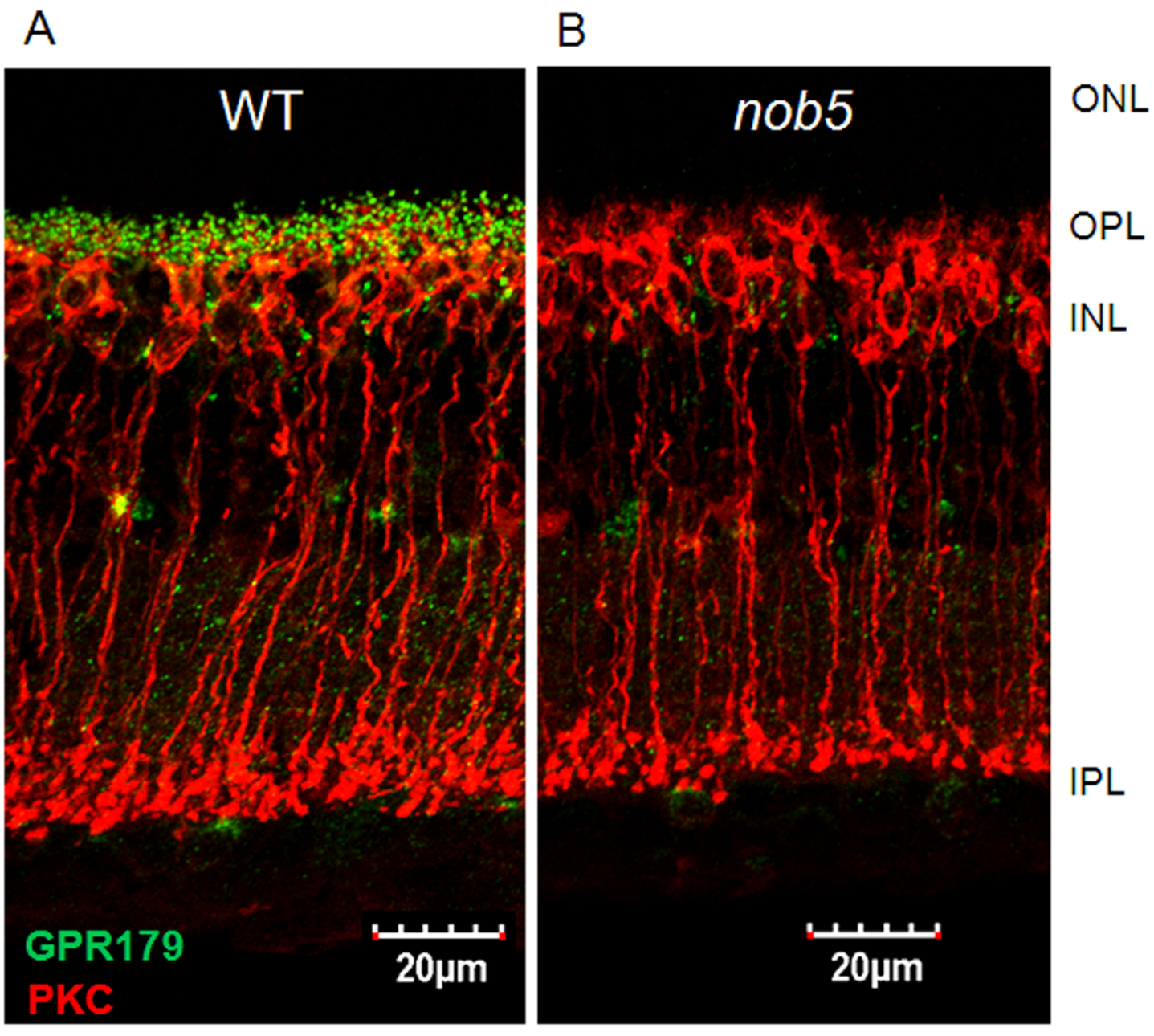

Figure 13: GPR179 immunostaining in WT and nob5 retinal slices. (A) WT retinas were labeled with antibodies against PKC (red) and GPR179 (green). WT retinas show robust labeling of GPR179 in the OPL. (B) In contrast, nob5 retinas are devoid of GPR179 staining in the OPL. 


\section{GPR179 is expressed at the tips of the DBCs}

To determine a more precise localization for GPR179 expression I co-labeled retinas with antibodies against GPR179 and several that label other cell type and have dendrites or axon terminals in the OPL. The horizontal cells form invaginating synapses with rod and cone photoreceptors, along with the ON bipolar cells (Kolb, 1970). To determine if GPR179 was expressed in the horizontal cells of the retina I double labeled retinal sections for GPR179 and calbindin, a horizontal cell marker. The staining shows GPR179 expression is juxtaposed to calbindin staining in the OPL, but they do not colocalize (Fig. 14A). This result indicates that GPR179 is not expressed in the horizontal cells (Fig. 14A).

To determine if GPR179 is expressed in the Müller cell processes in the OPL retinas were double labeled for GPR179 and glutathione synthetase (GS) a Müller cell marker. Müller cells have processes throughout the INL and OPL that aid in uptake and release of neurotransmitters, ions and other metabolic molecules (Bringmann et al., 2006; Bringmann et al., 2009; Agte et al., 2011). The immunostaining shows that GPR179 expression is restricted to the tips of the DBCs in the OPL and does not colocalize with GS (Fig. 14B). There also is no GPR179 staining in the vicinity of the Müller cell endfeet (Fig. 14B).

GPR179 was expected to be expressed post synaptically on the DBCs. The ISH data along with the normal ERG a-wave and absent b-wave suggested the protein was expressed in the DBCs. To further test this hypothesis I double labeled fixed transverse retinal sections with the presynaptic marker RIBEYE, which stains ribbon synapses, and GPR179 (Fig. 14C). RIBEYE is expressed pre-synaptically in the OPL and forms a "horseshoe" shaped pattern at the axon terminals of rod BCs. The immunostaining shows that GPR179 and RIBEYE do not colocalize, but appose one another, indicating 
that GPR179 is expressed post synaptically (Fig. 14C) and (Orlandi et al., 2012; Peachey et al., 2012b). 


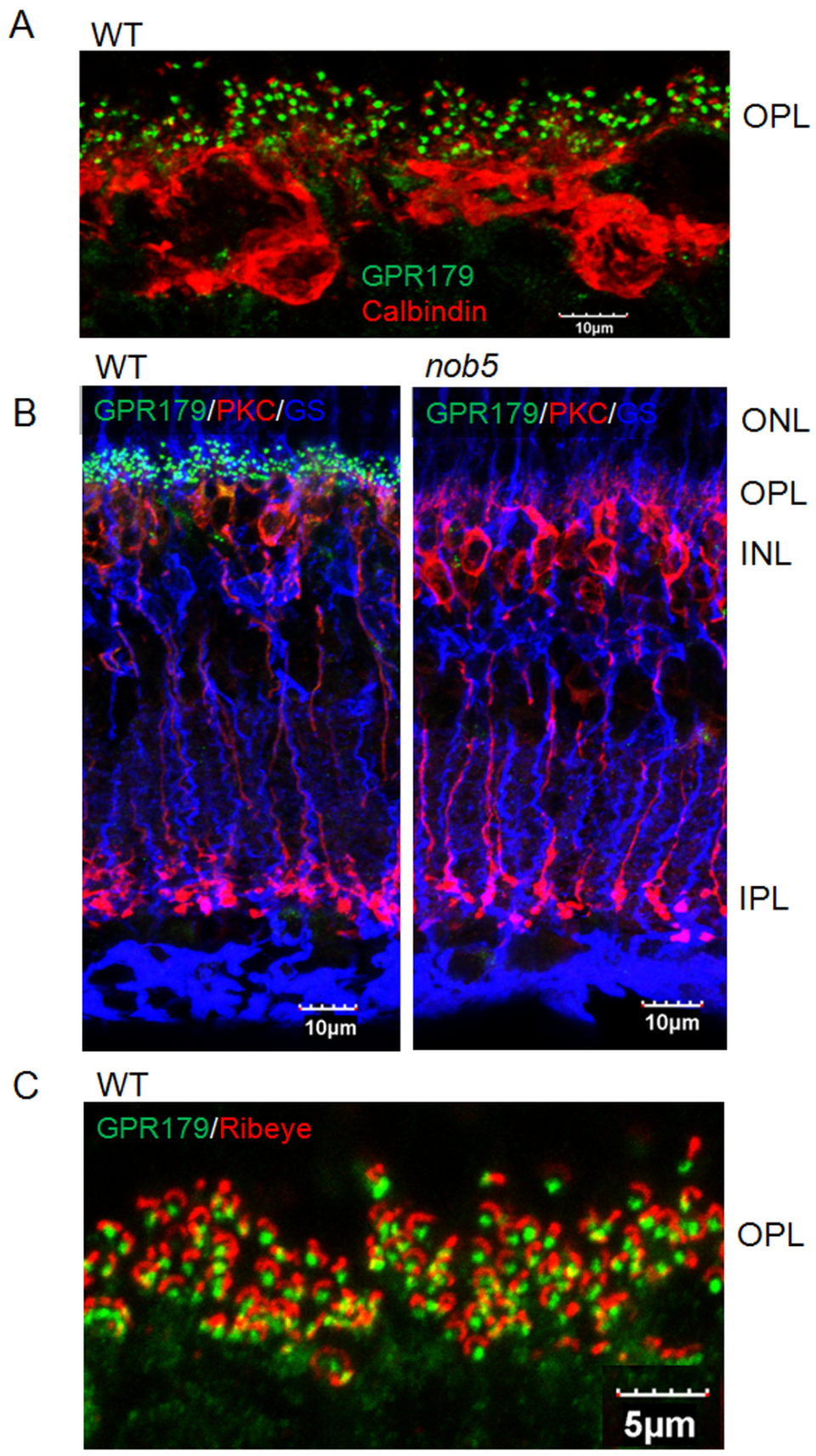

Figure 14: GPR179 is expressed post synaptically at the tips of the DBCs. (A) WT retinas are labeled with antibodies against GPR179 (green) and calbindin (red). Visualization of labeled proteins shows GPR179 and calbindin staining do not colocalize. (B) WT retinas are double labeled with antibodies against GPR179 (green), PKC (red) and GS (blue). The PKC staining localizes the GPR179 puncta at the tips of the rod BCs in the OPL. GPR179 labeling does not colocalize with GS labeling indicating that GPR179 is not expressed in the müller cells. (C) WT retinas are double labeled with antibodies against GPR179 (green) and RIBEYE (red). The RIBEYE staining localizes GPR179 to the postsynaptic side of the synapse. 
If GPR179 is expressed on the tips of the DBCs it should colocalize with mGluR6. Double labeling transverse retinal sections with antibodies against GPR179 and mGluR6 followed by confocal microscopy shows robust colocalization in the OPL (Fig 15). Because mGluR6 is known to colocalize with TRPM1 (Koike et al., 2010b) and TRPM1 with nyctalopin (Pearring et al., 2011), GPR179 is expected to colocalize with nyctalopin and TRPM1. These data support the hypothesis that GPR179 is expressed at the tips of the DBCs and is part of the postsynaptic signalplex, which is the supramolecular complex of proteins involved in signal transmission. 


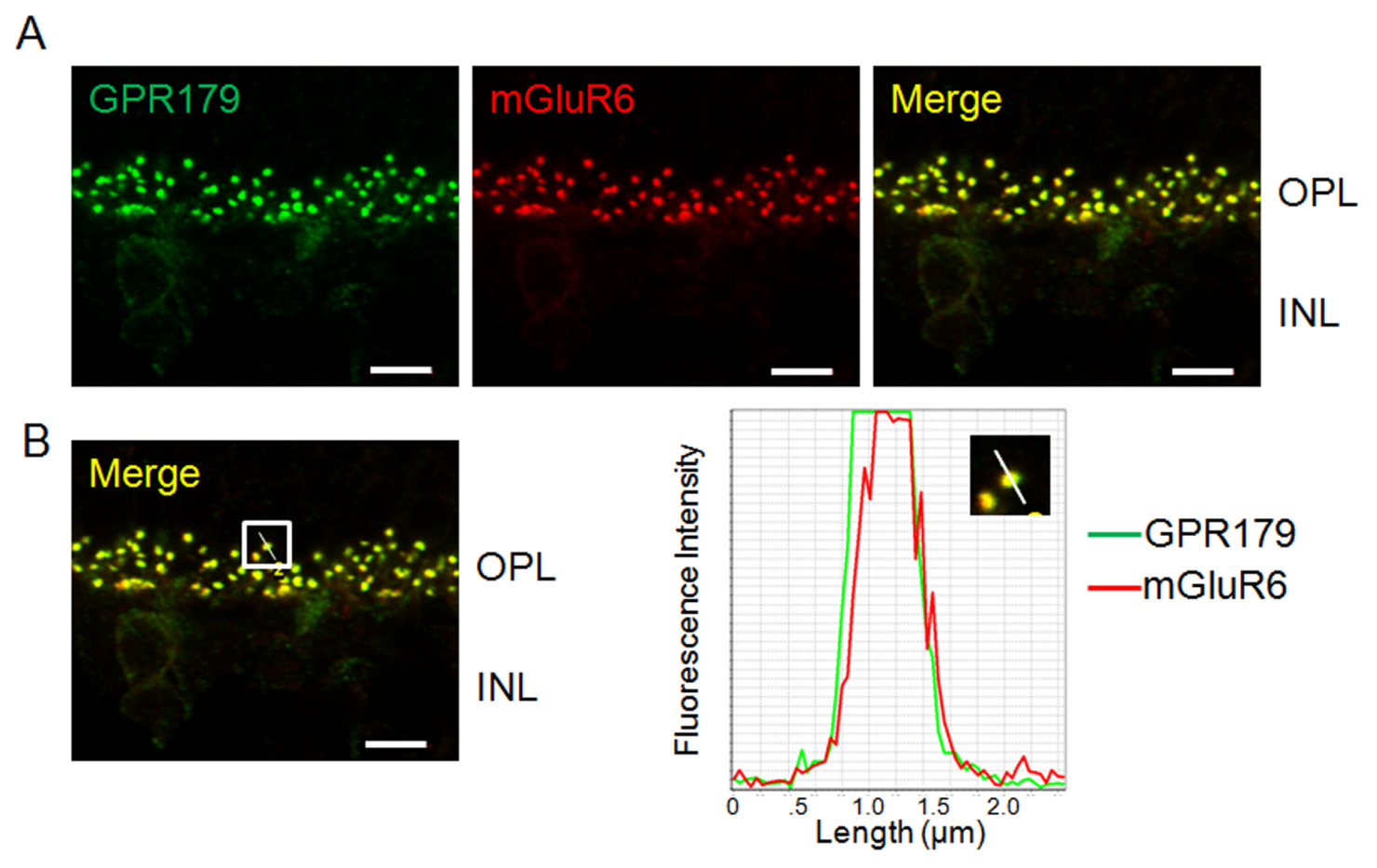

Figure 15: GPR179 colocalizes with mGluR6. (A) WT transverse retinal sections were labeled with antibodies against GPR179 (green) and mGluR6 (red) and visualized using confocal microscopy. GPR179 puncta appear to overlap with all mGluR6 puncta. (B) A $2.4 \mu \mathrm{m}$ line was drawn through a puncta and fluorescence was measure across the line. The GPR179 signal colocalizes with mGluR6. Scale bar represents $5 \mu \mathrm{m}$. 


\section{Morpholino Knockdown of GPR179 greatly reduces ERG b-wave}

To determine the effect of disruption of GPR179 protein translation on zebrafish vision, we used morpholinos to knockdown GPR179 expression. Morpholinos are antisense oligomers made complementary to an mRNA sequence that interferes with either protein translation or RNA processing (Bill et al., 2009).

GPR179 blocking morpholinos (Gene Tools, Philomath, OR) were injected into single cell embryos and ERGs were recorded 4-6 days post injection. The zebrafish injected with GPR179 blocking morpholinos show a significantly decreased b-wave compared to those injected with scrambled morpholino at all flash intensities (Fig. 16) and (Peachey et al., 2012b). Morpholinos against nyctalopin were injected as a positive control (Bahadori et al., 2006) and show a reduced b-wave, similar in magnitude to the morpholinos directed against GPR179 (Fig. 16) (Peachey et al., 2012b). 
A

MO-gpr179

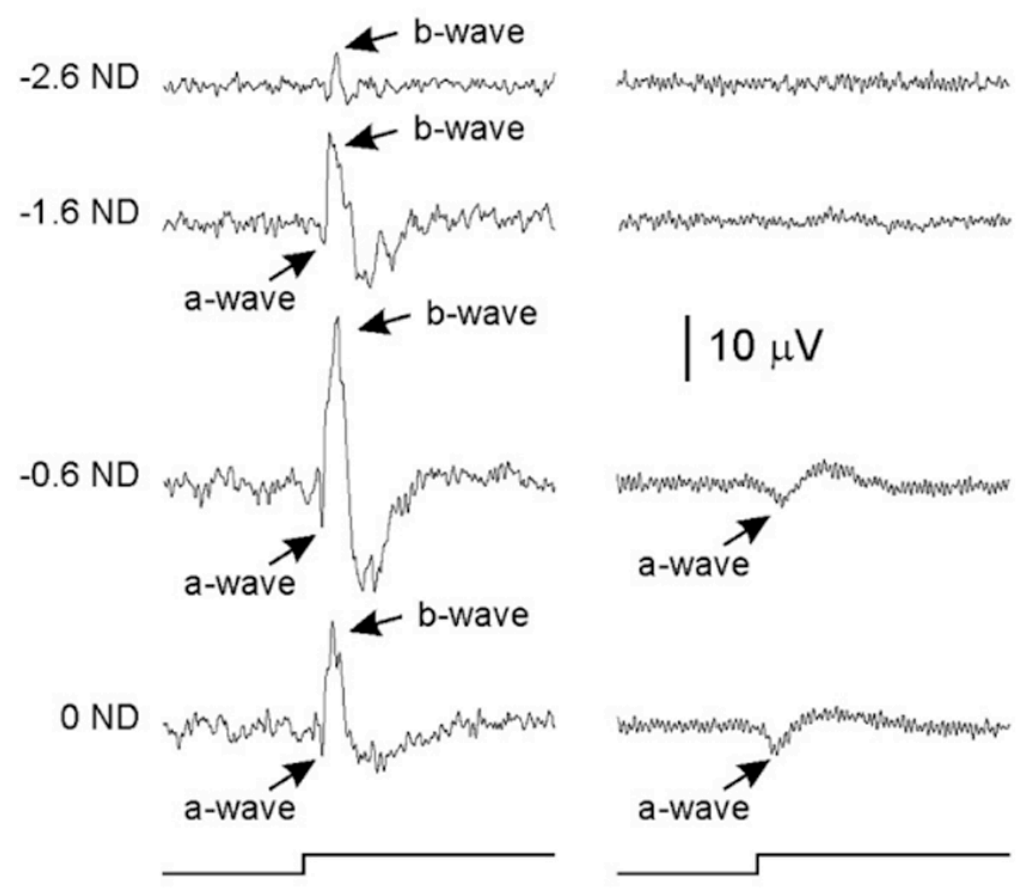

B

Control MO-gpr179 MO-NYX

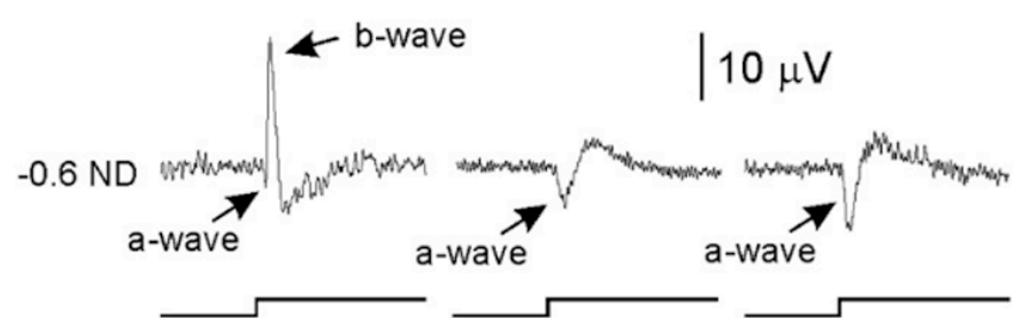

Figure 16: Morpholino knockdown of GPR179 in zebrafish. (A) ERG responses to various flash intensity stimuli from zebrafish injected with scrambled morpholinos (left). ERG responses to various flash intensity stimuli injected with morpholinos against GPR179 (right). ERG b-wave is severely decreased in zebrafish injected with morpholinos against GPR179 compared to scrambled morpholinos. (B) Morpholinos against nyctalopin cause a similar decrease in b-wave amplitude (Peachey et al., 2012b) 


\section{Human CSNB patients identified with mutations in Gpr179}

To determine if human cCSNB patients had mutations in GPR179 our collaborators screened all 11 exons of Gpr179 in 44 patients with cCSNB of unknown cause. Two unrelated probands were identified with putative inactivating mutations (Fig. 17B,C) (Peachey et al., 2012b). The first proband was a compound heterozygote that carried two frameshift mutations, pLeu63Serfs*12 and pSer329Leufs* 4 , terminating translation (Fig. 17B). The second proband had the pSer329Leufs ${ }^{*} 4$, the same as proband 1 on one allele and a missense mutation pTyr220Cys on the other allele (Fig. 17C). The missense mutation changes an amino acid in a highly conserved region of the protein and is predicted to be a null allele (Fig. 17D). Neither of the probands had other known health problems. In addition to the two patients our collaborators identified, Catherine Zeitz's group identified five cCSNB patients with mutations in Gpr179 (Audo et al., 2012). 

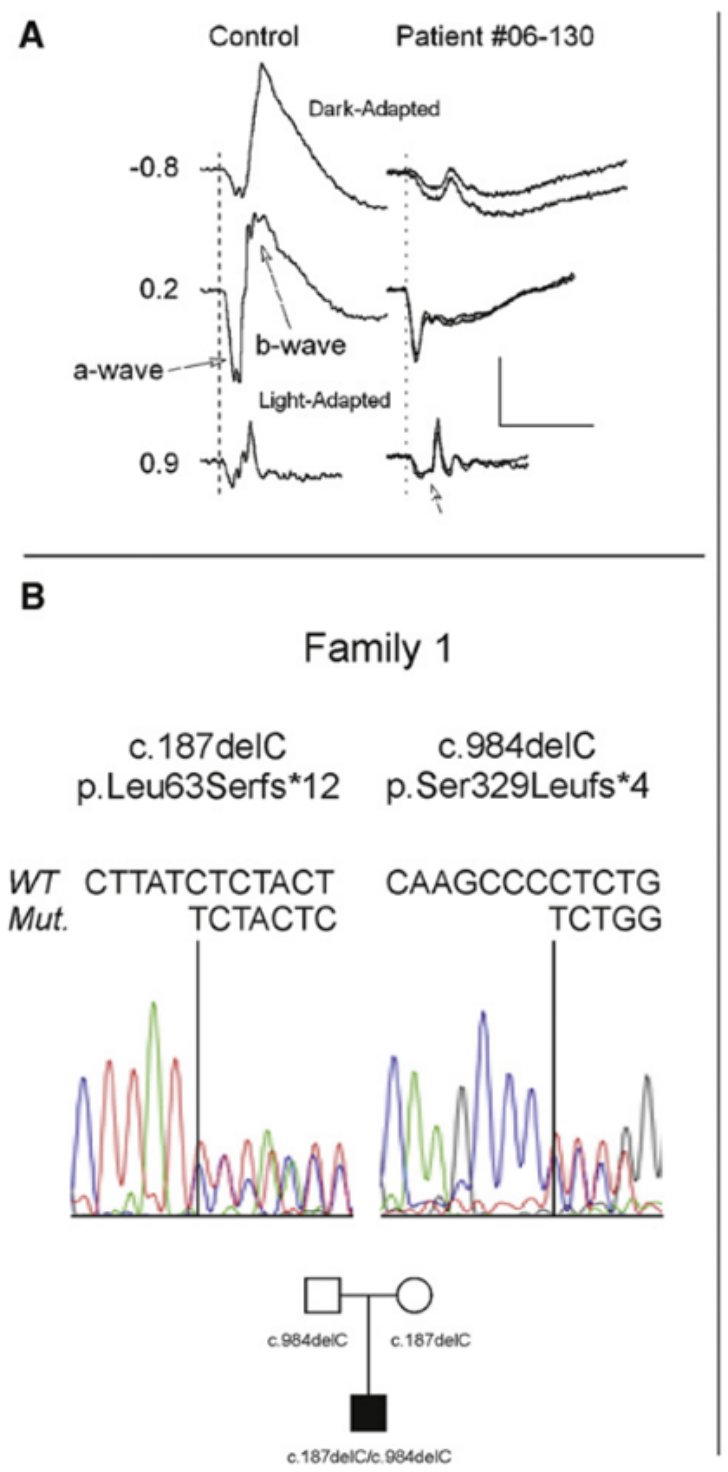

C

Family 2

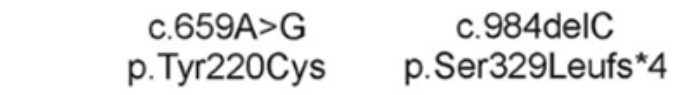

WT ATG GAT ATG TGG CAAGCCCCTCTG

Mut.

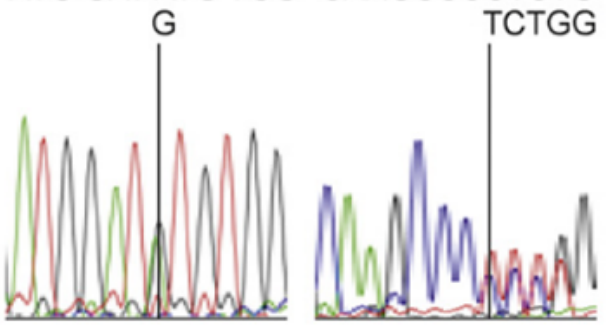

D
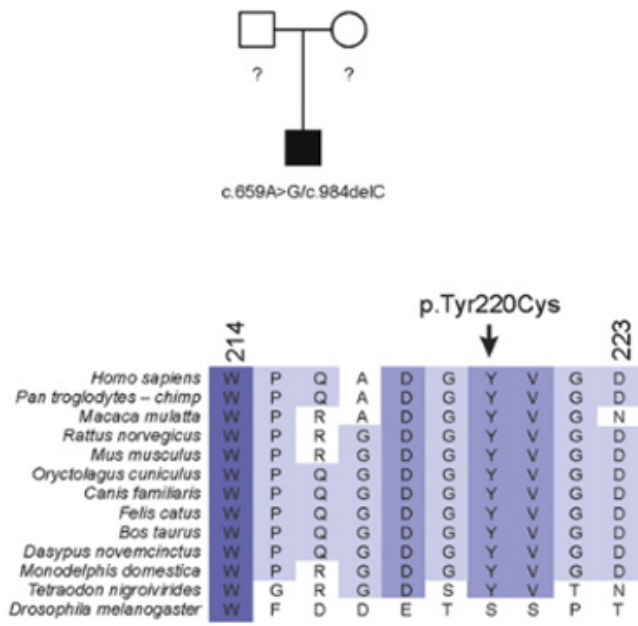

Figure 17: Human cCSNB patients with mutations in Gpr179. (A) ERGs from patient with cCSNB and mutations in Gpr179. (B) Proband 1 is a compound heterozygote with two frameshift mutations. (C) Proband 2 is a compound heterozygote with frameshift mutation and a missense mutation. (D) The missense mutation changes an amino acid in a highly conserved region of the protein. From (Peachey et al., 2012b). 


\section{GPR179 knockout}

The Gpr179 nob5 mouse is an insertion mutation and there is the possibility that a small amount of normal splicing does occur, making this a hypomorphic allele. To determine if the Gpr179 ${ }^{\text {nob5 }}$ mouse is a null we will compare the phenotype to a Gpr179 knockout mouse. A Gpr179 knockout mouse is currently in the pipeline. Embryonic stem cells containing a conditional knockout allele were available from the University of California Davis Knockout Mouse Project (KOMP) Repository. The Gpr179 $9^{\text {tm1e(KOMP)Mbp }}$ have conditional potential because LoxP sites were engineered into the gene and flank exon 2. The deletion of exon 2, which encodes 109 nucleotides in the gene will cause a frameshift mutation, resulting in a truncated protein, which would most likely undergo nonsense-mediated decay. The ES cells were purchased and used to create a mouse line at the University of Cincinnati. The colony is currently being established at the University of Louisville. We currently have 1 viable mouse carrying the knockout allele that we are breeding. Once the knockout allele is made homozygous, an ERG will be performed to confirm GPR179 is critical for DBC function.

\section{Discussion}

It was previously thought that the major components in the DBC signaling cascade had been identified such as the glutamate receptor mGluR6 and the cation channel TRPM1. Much of the recent effort has been to identify the molecules that are activated by the mGluR6 receptor such as $\mathrm{G}$ proteins and second messengers that are responsible for gating of the TRPM1 channel (Okawa et al., 2010; Dhingra et al., 2012; Shen et al., 2012). In this chapter I have shown that a novel GPCR, GPR179, is the likely cause of cCSNB in human patients and is mutated in a new model of cCSNB (Peachey et al., 2012b). The discovery of a second GPCR in the system adds another level of complexity to the signaling mechanism and appears to be quite unique among GPCR cascades. 
GPR179 is classified as a class C orphan GPCR based on sequence identity (Rondard et al., 2011). Despite this categorization, it does not share the typical characteristics of other Class C receptors. In silico analyses of GPR179 shows it lacks the extracellular venus fly trap (VFT) ligand binding domain that the vast majority of class C GPCRs possess (Fig. 18), including mGluR6 (Soding, 2005). The VFT domain is required for ligand binding and plays a role in receptor dimerization (Ray et al., 1999; Awatramani and Slaughter, 2000; Kennedy et al., 2004). The extracellular amino terminus of GPR179 contains a calcium-binding EGF-like domain of unknown function. This domain is commonly found in membrane bound or extracellular proteins that require calcium for their tertiary protein structure, which is thought to facilitate protein interactions (Selander-Sunnerhagen et al., 1992).

The carboxy terminus IC domain of GPR179 is unique among Class C receptors. The IC domain is large ( 1700 amino acids) and composed of a highly repetitive amino acid sequence CPWE that is conserved across species. This sequence does not share homology with other known proteins; therefore, the function is unknown. The tertiary structure of the carboxy terminus of GPR179 is predicted to be disordered and contain several internal repeats (Letunic et al., 2012). The IC loops of GPR179 are predicted to couple to $\mathrm{G}_{\mathrm{i} / \mathrm{o}} \mathrm{G}$ proteins (Sgourakis et al., 2005a; Sgourakis et al., 2005b), potentially allowing it to activate $\mathrm{G}$ proteins in the DBCs.

Interestingly, none of the Gpr179 mutations reported to date in patients with cCSNB reside in the carboxy terminus (Audo et al., 2012; Peachey et al., 2012b), which comprises $73 \%$ of the amino acid sequence. Perhaps mutations in the carboxy terminus of GPR179 that inhibit function are more rare because the it is highly redundant and has enough structural flexibility that a missense or nonsense mutation is unlikely to adversely affect function. This suggests that the extracellular amino terminus is critical to function because mutations in the extracellular amino terminus cause cCSNB. 


\section{GPR179}

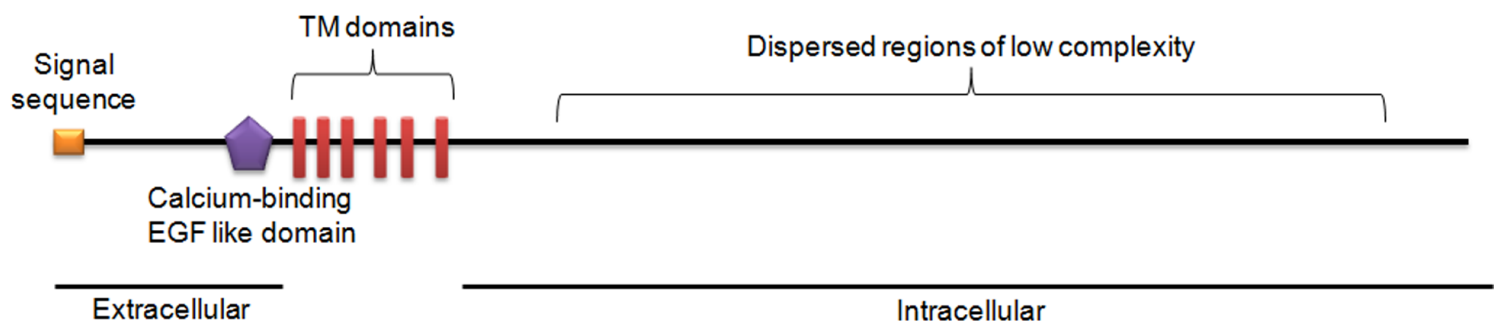

Figure 18: Protein domains of GPR179. GPR179 is predicted to be a 7 TM protein with an extracellular calcium-binding EGF like domain. The large cytoplasmic carboxy terminus of GPR179 contains intermittent regions of low complexity. Domains predicted using SMART 7 (Letunic et al., 2012). 
There are a number of important directions for future studies on GPR179. One direction that needs to be explored is the expression profile of Gpr179 in other tissues. Despite the fact that nob5 shows no other phenotypes and human patients with mutations in Gpr179 are healthy (Audo et al., 2012; Peachey et al., 2012b), it is possible that Gpr179 is expressed in other tissues and cell types. Finding Gpr179 in other cell types may allow a tissue culture system to be utilized with endogenous expression of Gpr179, allowing its function to be more easily studied.

In conclusion, we mapped the mutation responsible for DBC dysfunction in the nob5 mouse. Using next generation sequencing we identified a new gene, Gpr179, critical to glutamate signaling in the DBCs. We found human patients with cCSNB carrying mutations in GPR179, validating our hypothesis that Gpr179 is critical to DBC function and we localized mRNA and protein expression to the DBCs using ISH and immunohistochemistry. A Gpr179-- mouse was created to confirm Gpr179 is the cause of DBC dysfunction in the nob5 mouse and that the nob5 mouse is not a hypomorph.

\section{Methods}

\section{Animals}

All procedures involving animals were performed in accordance with the policies on the use of animals in research and each local Institutional Animal Care Use Committees. Descriptions of all mice used have been published previously (Masu et al., 1995; Pardue et al., 1998; Gregg et al., 2007; Pearring et al., 2011; Cao et al., 2012;

Peachey et al., 2012b) and each line was either generated on, or backcrossed onto a C57BL6/J background for at least 6 generations. All mice were housed in local AAALAC approved facilities under a $12 \mathrm{~h}$ light/12 h dark cycle. Mice were euthanized by $\mathrm{CO}_{2}$ inhalation followed by cervical dislocation.

\section{DNA isolation}


For mouse genotyping, $5 \mathrm{~mm}$ tail biopsies were collected from mice and placed in $0.7 \mathrm{~mL}$ microcentrifuge tube, and lysed with $200 \mu \mathrm{L}$ of DirectPCR (Viagen Biotech) containing $2 \mu \mathrm{L}$ proteinase $\mathrm{K}(20 \mathrm{mg} / \mathrm{ml})$. Samples were incubated for at least $4 \mathrm{~h}$ at $55^{\circ} \mathrm{C}$ in a dry bath with brief vortexing every h. After the tail sample was adequately digested, proteinase $\mathrm{K}$ was inactivated by heating to $85^{\circ} \mathrm{C}$ for $30 \mathrm{~min}$. Samples were then ready for $P C R$.

For next generation sequencing, DNA was isolated from mouse liver. Liver samples were collected from mice and placed into a $1.5 \mathrm{~mL}$ microcentrifuge tube and digested in $300 \mu \mathrm{l}$ of cell lysis solution (Qiagen) plus $3 \mu$ proteinase $\mathrm{K}(20 \mathrm{mg} / \mathrm{ml})$. Samples were incubated at least $4 \mathrm{~h}$ and vortexed until fully digested. Protein was precipitated from solution using protein precipitation solution (Qiagen) and DNA was recovered by ethanol precipitation.

\section{General Polymerase Chain Reaction}

Polymerase Chain Reaction (PCR) was generally performed using Phire II or Phusion taq polymerase (Thermo Scientific). Reactions were made to $20 \mu$ l consisting of $0.5 \mu \mathrm{M}$ of each forward and reverse primers, $200 \mu \mathrm{M}$ of dNTPs, $4 \mu \mathrm{l}$ of $5 \mathrm{X}$ reaction buffer, and either $0.4 \mu \mathrm{l}$ of Phire II or Phusion DNA Polymerase. Template DNA amount and water varied by application. Reactions were run on a Bio Rad C1000 Touch Thermal Cycler.

\section{High Resolution Melting analysis}

High Resolution Melting (HRM) analyses were performed according to Applied Biosystems High Resolution Melting Getting Started Guide (Applied Biosystems). Briefly, primers were designed by the web program Primer3 (Untergasser et al., 2012) to flank a SNP and produce a 60-100bp amplicon product. Reactions were set up in 96well Fast reaction plates with a $10 \mu \mathrm{l}$ reaction volume consisting of $5 \mu \mathrm{m}$ of each forward and reverse primers, $5 \mu$ l of MeltDoctor ${ }^{\mathrm{TM}} 2 \mathrm{X}$ HRM Master Mix (Applied Biosystems), 1 
$\mu \mathrm{l}$ of genomic DNA and PCR grade water to $10 \mu \mathrm{l}$. The reaction was run on a 96-well Fast plate on an Applied Biosystems 7900 HT Fast Real-Time PCR system. After PCR amplification DNA was denatured at $95^{\circ} \mathrm{C}$ and annealed at $37^{\circ \mathrm{C}}$ before high resolution melting at $1 \%$ ramp rate on a 96 -well Fast block. Melting curves were analyzed using HRMv2.0.1 software.

\section{Next Generation Sequencing}

Samples were prepared using the Illumina protocol "Preparing Samples for Paired-End Sequencing (Part\# 1005063 Rev. A June 2008). Genomic DNA samples were sheared to an average size of $\sim 300 \mathrm{bp}$ using sonication. DNA fragment ends were repaired and phosphorylated using Klenow, T4 DNA Polymerase and T4 Polynucleotide Kinase. An adenine base was added to the 3' end of the blunted fragments, followed by ligation of Illumina Paired-End adapters via T-A mediatied ligation. The library was hybridized to the sequence capture array on a Nimlegen Hybridization System 4 for $\sim 64$ $\mathrm{h}$ at $42^{\circ} \mathrm{C}$. The array was washed to remove non-hybridized DNA and captured DNA was eluted using the Nimblegen Elution System. The eluted DNA was amplified by PCR using Illumina Paired-End primers and size selected for $300 \mathrm{bp}$ fragments via gel electrophoresis. The library size and concentration were determined using an Agilent Bioanalyzer.

\section{RNA isolation from Retina}

Mice were euthanized by $\mathrm{CO}_{2}$ inhalation and cervical dislocation. Eyes were extracted and enucleated and the retinas were removed and stored at $-80^{\circ} \mathrm{C}$ in RNAlater (Ambion) until needed. RNA was isolated according to manufacturers protocol for the PureLink® RNA Mini Kit (Ambion). Briefly, retinal tissue was removed from RNAlater and homogenized in 10-20 volumes of Tri Reagent (Ambion) by pipetting. The homogenized mixture was incubated for $5 \mathrm{~min}$ at room temperature then $200 \mu \mathrm{l}$ chloroform per $\mathrm{ml}$ of homogenate was added and the mixture vortexed, and incubated 
for an additional $5 \mathrm{~min}$ at room temperature; followed by centrifugation at $12,000 \times \mathrm{g}$ for $10 \mathrm{~min}$ at $4^{\circ} \mathrm{C}$. The aqueous phase volume was transferred to a new $1.5 \mathrm{ml}$ centrifuge tube and $200 \mu \mathrm{l}$ of $100 \%$ ethanol was added to the sample and vortexed for $15 \mathrm{sec}$. The sample was placed in a filter cartridge (Ambion) and centrifuged at $12,000 \mathrm{xg}$ for $30 \mathrm{sec}$. Flow through was discarded and $500 \mu \mathrm{l}$ wash solution was applied to the filter cartridge and centrifuged at $12,000 \times \mathrm{g}$ for $30 \mathrm{sec}$. The wash step was repeated and the filter cartridge was transferred to a new microcentrifuge tube and $100 \mu \mathrm{l}$ of Elution buffer was applied to elute the RNA. The RNA was collected by centrifugation for $30 \mathrm{sec}$ at 12,000 $x$ g. The RNA was stored at $-80^{\circ} \mathrm{C}$.

\section{Antibodies}

The following primary antibodies and their concentrations were used: sheep antiGPR179 (peptide KVQEETPGEDLDRPVLQKR), 1:2,000 (Peachey et al., 2012b); mouse monoclonal anti-ctbp2/Ribeye (BD Bioscience), 1:1,000; guinea pig anti-mGluR6 1:1,000 (Koike et al., 2010b); sheep anti-TRPM1 1:1,000 (Cao et al., 2011); rabbit antiGFP (MBL), 1:800 and Rhodamine Peanut Aglutinin (PNA) conjugate 566 (Vector Labs), 1:1,000; goat anti-Gß5 (peptide MATDGLHENETLASLKC), 1:1,000 (Morgans et al., 2007); goat anti-R9AP 1:1,000 (Hu and Wensel, 2002); mouse anti-DIG HRP, 1:1,000 (Perkin Elmer); mouse anti-GFP, 1:1,000 (Molecular Probes); sheep anti-RGS11 (peptide CSPALQSTPREPAATSSPEGADGE), 1:1,000 (Martemyanov et al., 2005); sheep anti-RGS7 1:1,000 (Morgans et al., 2007); mouse anti-RIBEYE 1:1,000 (BD Transduction Labs); rabbit anti-PKC 1:1,000 (Sigma Aldrich).

Secondary antibodies (Invitrogen, 1:1,000) appropriate to each primary antibody included: donkey anti-sheep Alexa-488, donkey anti-rabbit Alexa-680, donkey anti-rabbit Alexa-546, donkey anti-mouse Alexa-647 and donkey anti-guinea pig Cy3 (Millipore, $1: 1,000)$. In lieu of an antibody specific to nyctalopin, we used $T g(\text { Gabrr1-YFP/nyx })^{\text {Rgg1 }}$ 
transgenic mice that express a yellow fluorescent protein (YFP) tagged nyctalopin (Gregg et al., 2007). They are labeled WT in the figures.

\section{Retinal dissections for in situ hybridization}

Mice were sacrificed by carbon dioxide gas inhalation followed by cervical dislocation. The eyes were enucleated and the cornea, iris and lens were removed and the eyecup was rinsed in ice cold PBS-Diethylpyrocarbonate (Diethylpyrocarbonate (DEPC) was added to PBS at a dilution of $1: 1,000$, solution was mixed vigorously, incubated overnight then autoclaved). Eyecups were immediately fixed in $4 \%$ formaldehyde PBS-DEPC for $1 \mathrm{~h}$ at room temperature. Eyecups were then washed twice in PBS-DEPC then cryoprotected in $15 \%$ sucrose PBS-DEPC at $4^{\circ} \mathrm{C}$ until sucrose fully penetrated and were then moved into a $30 \%$ sucrose PBS-DEPC solution at $4^{\circ} \mathrm{C}$ overnight.

\section{DIG labeling RNA}

DIG RNA labeling was performed as described in the Roche DIG RNA Labeling Kit (SP6/T7). In brief, purified template DNA $(1 \mu \mathrm{g})$ was added to a sterile, RNase-free PCR tube. The volume was brought to $13 \mu$ with sterile, RNase free DEPC treated water. To the reaction tube, $2 \mu$ of $10 X$ NTP labeling mixture, $2 \mu$ of $10 X$ Transcription buffer, $1 \mu \mathrm{l}$ of Protector RNase inhibitor, and either SP6 or T7 RNA Polymerase (2 $\mu \mathrm{l})$ were added and mixed gently. Reaction was incubated at $37^{\circ} \mathrm{C}$ for $2 \mathrm{~h}$ on a PCR thermal cycler. DNase I $(2 \mu \mathrm{l})$ was added to the reaction tube and incubated for $15 \mathrm{~min}$ at $37^{\circ} \mathrm{C}$. RNA was analyzed by native agarose gel electrophoresis. The reaction volume was brought to $100 \mu \mathrm{l}$ with sterile, RNase free, DEPC treated water containing $10 \mathrm{mM}$ DTT and stored at $-80^{\circ} \mathrm{C}$.

\section{In situ hybridization}

Day One 
Slides were removed from $-80^{\circ} \mathrm{C}$ storage and dried at $65^{\circ} \mathrm{C}$ in hybridization oven for $30 \mathrm{~min}$. Sections were fixed for $20 \mathrm{~min}$ at $-20^{\circ} \mathrm{C}$ in methanol then washed three times for 5 min each with PBST-DEPC (PBS-DEPC $+0.1 \%$ tween twenty). Slides were incubated in proteinase $\mathrm{K}$ solution $(1.2 \mu \mathrm{g} / \mathrm{ml}$ Proteinase $\mathrm{K}$ in $50 \mathrm{mM}$ Tris- $\mathrm{HCl} \mathrm{pH} 7.5+$ $5 \mathrm{mM}$ EDTA) for $12 \mathrm{~min}$, washed in PBST for $5 \mathrm{~min}$ and fixed again with $4 \%$ formaldehyde PBS-DEPC for 5 min. Sections were rinsed in PBST-DEPC then washed 2 more times for $5 \mathrm{~min}$. Sections were then acetylated in acetylation solution $(73.75 \mathrm{~mL}$ $\mathrm{H}_{2} 0$-DEPC, $1 \mathrm{~mL}$ Triethanolamine, $135 \mu \mathrm{L} \mathrm{HCl}$ and $188 \mu \mathrm{L}$ acetic anhydride). Sections were washed in PBST-DEPC 3 times for 5 min then incubated in PBST-DEPC $+3 \%$ $\mathrm{H}_{2} \mathrm{O}_{2}$ for 30 min. Sections were air dried for $\sim 10$ minutes. Subsequently hybridization solution was removed from the freezer and brought to $65^{\circ} \mathrm{C}$. DIG RNA probes were thawed on ice. Probes were diluted 1:100 in $100 \mu \mathrm{L}$ of hybridization solution and heat denatured for $10 \mathrm{~min}$ at $85^{\circ} \mathrm{C}$. Sections were placed in hybridization chamber that was dampened with $50 \%$ formamide/5X SSC. Probe solution was removed from heat and $100 \mu \mathrm{L}$ was placed directly on slide and a glass coverslip was added to slide. Sections were incubated overnight in a hybridization oven at $65^{\circ} \mathrm{C}$.

Day Two

The coverslip was removed by immersing the slide in 2X SSC and slides were incubated in fresh $2 \mathrm{X} \mathrm{SSC}$ for $20 \mathrm{~min}$ at $65^{\circ} \mathrm{C}$, then were washed in coplin jars containing $0.2 \mathrm{XSSC}$ at $65^{\circ} \mathrm{C}$ over a period of $3-4 \mathrm{~h}$, changing the solution every $30 \mathrm{~min}$. Slides were then washed 3 times for 5 min in maleic acid buffer supplemented with $0.1 \%$ Tween-20 (MABT, pH 7.5) then incubated for $1 \mathrm{~h}$ in MABT $+5 \%$ normal donkey serum. Mouse anti DIG HRP antibody was diluted 1:1000 and added to slides and incubated overnight in humidity chamber at $4^{\circ} \mathrm{C}$. If double labeling with a cell marker antibody, it was applied to the solution at this time.

Day Three 
Slides were washed $3 \times 10 \mathrm{~min}$ in PBST. Cy3-TSA staining solution (Roche) was prepared at 1:100 dilution and immediately added to slides and incubated for 50 min. Sections were washed $3 \times 10 \mathrm{~min}$ in PBST. If fluorescent staining for a cell marker, dilute fluorescent antibody was incubated for $1 \mathrm{~h}$ at room temperature then was $3 \times 10 \mathrm{~min}$ in PBST. Slides were mounted with glass coverslips using Immu-Mount (Thermo Scientific) and imaged on a FV-1000 confocal microscope.

\section{Immunohistochemistry}

Mice were euthanized with $\mathrm{CO}_{2}$ followed by cervical dislocation. Their eyes were enucleated and the lens was removed. Eyecups were washed in phosphate buffered saline (PBS) then fixed for $30 \mathrm{~min}$ in a $4 \%$ paraformaldehyde PBS solution $(\mathrm{pH} 7.4)$. Eyecups were washed 3 times with PBS then cryoprotected in increasing concentrations of sucrose in PBS $(10 \%, 15 \%$ for $1 \mathrm{~h}$ each and $30 \%$ overnight). Eyecups were embedded in 2:1 OCT/ $20 \%$ sucrose PBS solution frozen in a liquid nitrogen cooled bath of isopentane. Eyecups were sectioned $(18 \mu \mathrm{m})$ using a Leica 1850 cryostat. Sections were mounted on glass slides (SuperFrost) and allowed to dry for 10 minutes at room temperature before being stored at $-80^{\circ} \mathrm{C}$. Sections were air dried at $37^{\circ} \mathrm{C}$ for $15 \mathrm{~min}$ and washed with PBS and PBS containing $0.05 \%$ Triton X-100 (PBX) for 5 min each, then blocked in blocking solution (PBX plus 5\% normal donkey serum) for $1 \mathrm{~h}$. Sections were incubated overnight at room temperature in the presence of the primary antibody diluted in blocking solution, then washed 3 times for 10 min each with PBX followed by incubation in secondary antibody in blocking solution for $1 \mathrm{~h}$ at room temperature. Sections were washed $2 \times 10$ min in PBX and 1 X PBS. Slides were cover-slipped using Immu-Mount (Thermo Scientific) and slides were imaged using an Olympus FV1000 confocal microscope. Images were universally adjusted for brightness using Photoshop (Adobe). 


\section{CHAPTER III: CONSTRUCTING THE DEPOLARIZING BIPOLAR CELL SIGNALPLEX}

Aim 2: Determine signlaplex architecture and the role of GPR179 by identifying the interplay of DBC signalplex proteins in various mouse models of DBC dysfunction.

Rationale: All known mouse models of cCSNB have normal retinal structure (McCall and Gregg, 2008) but the synapse proteome is often affected by the loss of one or more proteins (Maddox et al., 2008; Jeffrey et al., 2010; Cao et al., 2011; Pearring et al., 2011; Orlandi et al., 2012; Xu et al., 2012). Many of the proteins expressed at the dendritic tips of the DBCs are dependent on expression of other proteins for trafficking or localization (Jeffrey et al., 2010; Cao et al., 2011; Pearring et al., 2011; Xu et al., 2012). Proteins codependent on one another for trafficking and localization often interact. To understand the arrangement of signalplex proteins in the DBCs, we can use immunohistochemistry and confocal microscopy to determine their interdependency for localization. Elucidating signalplex architecture is a step toward understanding the signaling mechanism that couples mGluR6 to the TRPM1 channel.

Hypothesis: GPR179 is a protein hub and is responsible for the recruitment and localization of signalplex proteins in the DBCs.

\section{Introduction}

The ability of cells to respond to external stimuli is fundamental to life and is possessed by all cells from the simplest of single cell organisms to all cells in vertebrates. At the most fundamental level, this ability requires the expression of a membrane receptor and its associated intracellular proteins. In neuronal systems, it is imperative that the postsynaptic neuron is able to faithfully relay the message from the 
presynaptic neuron, or when necessary, parse the neuronal code to relay a subset of the presynaptic message. To achieve this, the postsynaptic neuron must express a highly ordered and efficient signaling complex that captures the external signal and instructs the cell to respond in an appropriate manner.

The $~ 12$ types of $\mathrm{BCs}$ in the retina respond to light induced decreases in glutamate release from the photoreceptors in four general ways, a transient hyperpolarizing response, a sustained hyperpolarizing response, a transient depolarizing response and a sustained depolarizing response (Werblin and Dowling, 1969; Kaneko, 1970). Each of these responses requires a unique set of receptors and signaling proteins expressed at the plasma membrane of the post synaptic dendrite (Masu et al., 1995; DeVries and Schwartz, 1999; DeVries, 2000). Although physiologists have described the $\mathrm{BC}$ responses in detail, the molecular architecture of signaling proteins that give rise to these responses is largely unknown.

Rod BCs respond to a decrease in glutamate release from the photoreceptors with a transient depolarizing response (Dacheux and Raviola, 1986). Because $\sim 95 \%$ of the photoreceptors in the human retina are rods (Osterberg, 1937; Curcio et al., 1990) and rods only synapse with rod BCs, understanding how information is processed in the rod $\mathrm{BCs}$ denotes an understanding of how the majority of the ON BCs respond to light. Determining the interdependency of signalplex proteins should offer clues as to how the proteins are arranged at the synapse and how they may carry out signal transduction.

The glutamate receptor on the rod BCs is mGluR6 and binds glutamate, which activates a $\mathrm{G}$ protein cascade by promoting $\mathrm{Ga}_{\circ} \mathrm{GDP}$ to $\mathrm{Ga}_{\circ} \mathrm{GTP}$ exchange. Activation of $\mathrm{Ga}_{\circ}$ results in dissociation of the $\mathrm{G} \beta$ y complex. It is understood that one of the $\mathrm{G}$ protein subunits, $\mathrm{Ga}_{\circ}$ or $\mathrm{G} \beta y$, is responsible for gating the TRPM1 channel closed, by either direct interaction or though an effector protein. Recently, Shen et al. (2012) 
published that the Gßy dimer is responsible for gating the TRPM1 channel through direct interaction or an effector protein.

The RGS proteins, which inactivate GaGTP are known to be critical to DBC function but little is known about their molecular arrangement. The R7 family of RGS proteins (RGS6, RGS7, RGS9 and RGS11) are GTPase activating proteins (GAP) and are expressed almost exclusively throughout the nervous system. They are critical for setting the speed of G-protein signaling cascades in general because they speed up GaGTP to GaGDP hydrolysis (Koelle and Horvitz, 1996; Gold et al., 1997; Berman and Gilman, 1998; Burchett, 2000; Ross and Wilkie, 2000). The molecular arrangement of these proteins including interaction with a membrane anchor is critical to their function (Porter and Koelle, 2010). They are thought to organize into heterotrimeric complexes consisting of a membrane anchor protein, G 35 and the RGS protein (Cabrera et al., 1998; Anderson et al., 2009). There are two RGS membrane anchors expressed in DBCs, R9AP and R7BP (Cao et al., 2008; Jeffrey et al., 2010).

Several RGS knockout mouse models have been created to understand the arrangement and function of RGS proteins in the DBCs. The $R 9 A P^{-/-}, R 7 B P^{-/}$and

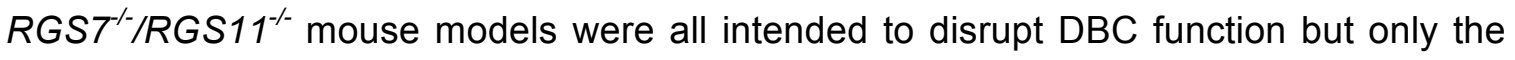
$R G S 7^{-/} / R G S 11^{-/}$mouse succeeded (Cao et al., 2008; Cao et al., 2009; Jeffrey et al., 2010; Cao et al., 2012). From these models we learned RGS7 and RGS11 are the main GAP proteins in the DBCs (Cao et al., 2012) but the membrane anchors R9AP and R7BP are not the only RGS anchors (Cao et al., 2008; Jeffrey et al., 2010). In the $R 9 A P^{-/}$retina, RGS11 did not localize properly at the dendritic tips of the DBCs but RGS7/Gß5 did (Jeffrey et al., 2010). R7BP deletion did not affect the localization of RGS7, RGS11 or G $\beta 5$ (Cao et al., 2008). These data indicate that in the DBCs there is new mechanism for RGS protein localization to the DBC dendritic tips or R7BP and R9AP are redundant to one another. 
To better understand the architecture of the DBC signaling proteins I used mutant mice with $\mathrm{DBC}$ dysfunction to examine synaptic protein localization using immunohistochemistry and confocal microscopy. The proteins mGluR6, GPR179, TRPM1, RGS11, RGS7, nyctalopin and R9AP were examined in all of the available mouse models of DBC dysfunction. Proteins that were co-dependent for localization at the DBC dendritic tips were examined for interaction using Duolink and Membrane Yeast Two-Hybrid (MYTH) assay. From these data, a model of signalplex protein arrangement was built.

\section{Results}

\section{GPR179 localizes to the DBC dendritic tips in mouse models of DBC dysfunction}

To determine if GPR179 is localized at the dendritic tips of the DBCs in the absence of other signalplex proteins, it was visualized by immunohistochemistry followed by confocal microscopy in cCSNB mouse models. Fixed transverse retinal

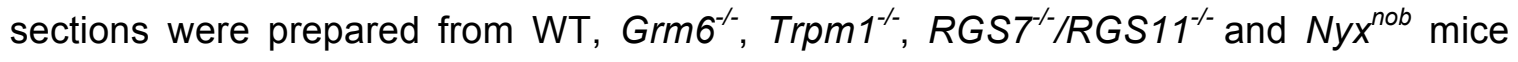
and were labeled with antibodies against GPR179 and the lectin peanut agglutinin (PNA). PNA labels the cone pedicles in the OPL and co-localizes with postsynaptic signalplex proteins on cone ON BCs (Blanks et al., 1988; Haverkamp et al., 2001). Visualization of the labeled proteins using confocal microscopy revealed that GPR179 is properly localized to the DBC dendritic tips in the $\mathrm{Grm} 6^{-/}, \operatorname{Trpm}^{-/-}, R G S 7^{-/ /} / \mathrm{RGS} 11^{-/}$and $N y x^{\text {nob }}$ retinas (Fig. 19). These data indicate that GPR179 is not dependent on mGluR6, TRPM1, RGS7/RGS11 or nyctalopin for proper localization at the DBC dendritic tips. It also indicates that protein localization of GPR179 is independent of normal DBC function. 


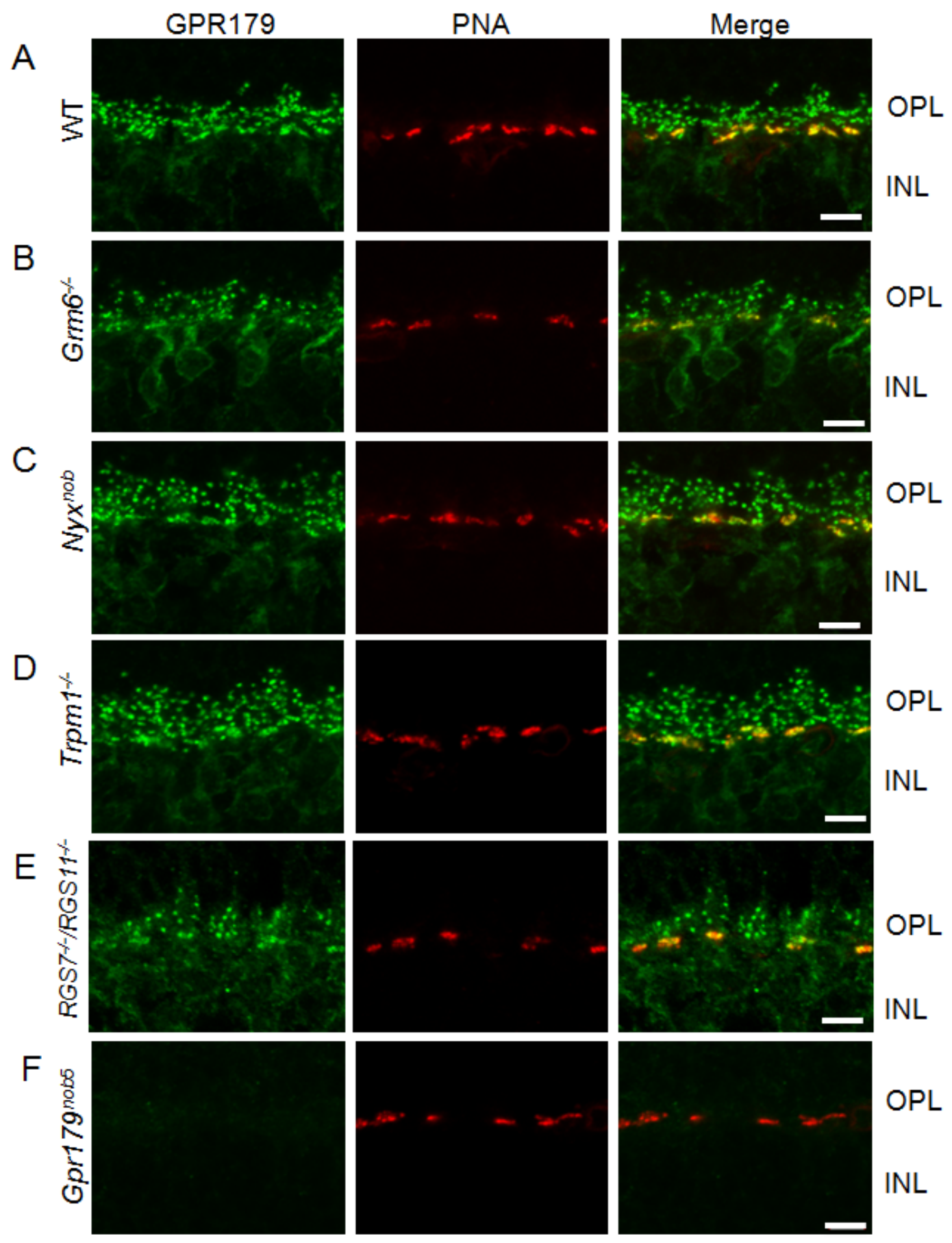

Figure 19: GPR179 expression in various mouse models of DBC dysfunction. (AE) GPR179 (green) localizes to the DBC dendritic tips and co-localizes with PNA (red) in the mouse models of DBC dysfunction (B) $\mathrm{Grm6}^{-/}$, (C) $\mathrm{Nyx}^{\mathrm{nob}}$, (D) $\mathrm{Trpm}^{-/-}$ and (E) $R G S 7^{-/ / R G S 11^{-/}}$. (F) GPR179 expression is absent in the Gpr179 ${ }^{\text {nob5 }}$ retina. 
The Gpr179 ${ }^{\text {nob5 }}$ mouse retina retains key signaling components including mGluR6, TRPM1 and nyctalopin

To determine the dependency of signalplex proteins on the expression of GPR179, I used the Gpr179 ${ }^{\text {nob5 }}$ mouse model, which lacks expression of GPR179 at the DBC dendritic tips (Fig. 17 and Peachey et al. (2012b)). Because we do not have a suitable antibody against nyctalopin for immunohistochemistry, I crossed the Gpr179 ${ }^{\text {nob5 }}$ with the $T g(G a b r r 1-Y F P / n y x)^{R g g 1}$ mouse, which expresses a yellow fluorescent protein (YFP) tagged nyctalopin (Gregg et al., 2007) to visualize nyctalopin expression in the Gpr179 nob5 mouse. Fixed transverse retinal sections from Gpr179 nob5 mice were prepared in conjunction with WT retinas and labeled with antibodies against mGluR6, TRPM1 and YFP-nyctalopin. The confocal microscopy revealed that mGluR6, TRPM1 and nyctalopin are properly localized on the DBC dendritic tips in the absence of GPR179 (Fig. 20). These data indicate GPR179 expression is not critical for the localization of mGluR6, TRPM1 and nyctalopin at the DBC dendritic tips. 


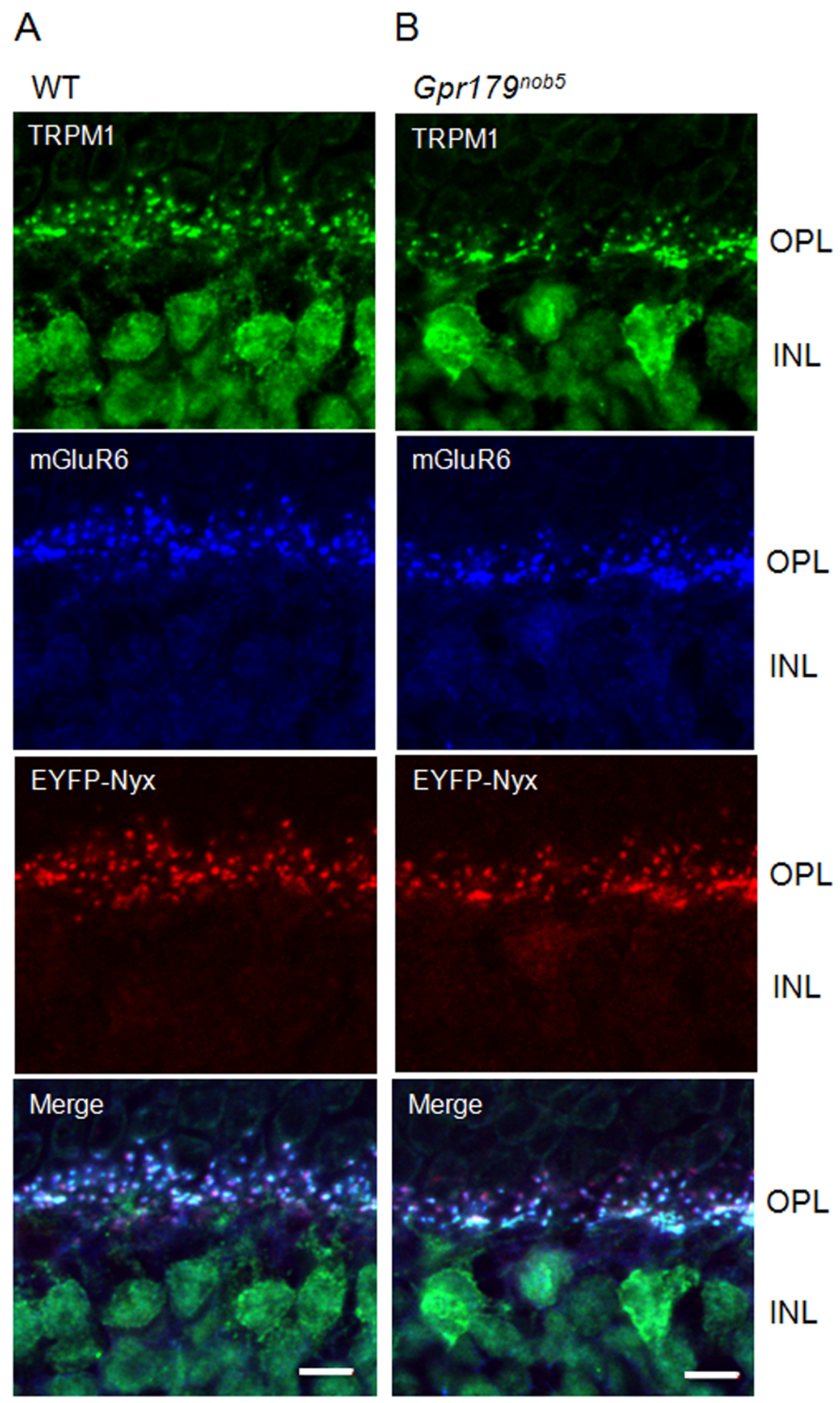

Figure 20: Expression of major signaling components in the Gpr179 ${ }^{\text {nob5 }}$ retina. Expression of TRPM1 (green), mGluR6 (blue) and EYFP-Nyx (red) in WT (A) and $\operatorname{Gpr179}^{\text {nob5 }}$ (B) retinas. Protein expression is not different compared to WT. Scale bar is $5 \mu \mathrm{m}$. From (Ray et al., 2013) 


\section{The absence of TRPM1 does not affect other signalplex proteins}

Localization of signalplex proteins was examined in $T r p m 1^{-/}$retinas. The proteins mGluR6 and nyctalopin are known to be expressed and localize correctly in the Trpm1 $^{-/}$DBCs (Pearring et al., 2011) and I showed that GPR179 expression and localization is not dependent on normal TRPM1 expression (Fig. 19). To determine if other signalplex proteins are dependent on TRPM1 expression for proper localization, immunohistochemical staining for RGS7, RGS11, R9AP, and Gß5 was performed on fixed transverse retinal sections from $\operatorname{Trpm}^{-/}$mice and visualized by confocal microscopy. In the Trpm $^{-/-}$retinas RGS7, RGS11, R9AP, and G $\beta 5$ all localized correctly to the DBC dendritic tips (Fig. 21), suggesting that they localize independently of TRPM1. Therefore, TRPM1, although critical for DBC function, does not play a critical structural role in the assembly of signalplex proteins.

\section{Nyctalopin is critical for TRPM1 localization}

From previous studies we know that TRPM1 is dependent on expression of nyctalopin for proper localization (Pearring et al., 2011) and the two proteins interact in vivo (Cao et al., 2011; Pearring et al., 2011). The mechanism of this dependency has not been resolved, therefore, I investigated the expression of mGluR6, GPR179, RGS7, RGS11, GB5 and R9AP in the $N y x^{\text {nob }}$ mice, which lack expression of nyctalopin (Pardue et al., 2001). Immunohistochemistry on fixed transverse retinal sections followed by confocal microscopy showed that the proteins mGluR6, GPR179, RGS7, RGS11, G 35 and R9AP all localized properly in the absence of nyctalopin (Fig. 22). These data indicate that nyctalopin plays a specific role in recruiting the TRPM1 channel to the DBC dendritic tips but is not important for the localization of mGluR6, GPR179, RGS7, RGS11, Gß5 or R9AP.

RGS7 and RGS11 deletion does not effect localization of TRPM1, GPR179 or mGluR6 
Using the $R G S 7^{-/} / R G S 11^{-/}$mouse model, which lacks expression of RGS7 and RGS11 in the DBCs, I examined the expression pattern of mGluR6, TRPM1, GPR179, R9AP and GB5. Immunohistochemical staining for mGluR6, TRPM1, GPR179, R9AP and G $\beta 5$ was performed on fixed transverse retinal sections from $R G S 7^{-/} / R G S 11^{-/}$mice then visualized using confocal microscopy (Fig. 22). I found that expression mGluR6, TRPM1 and GPR179 at the DBC dendritic tips was normal, and as expected G 35 expression was absent (Fig. 22 and Cao et al. (2012)). Surprisingly, R9AP expression was absent in the $R G S 7^{-/} / R G S 11^{-/} \mathrm{DBCs}$, suggesting that the formation of the complete RGS complex may be critical for complex localization.

\section{Localization of the RGS proteins to the DBC dendritic tips is lost in the Gpr179 nob5} mice

To determine if the absence of GPR179 affected the localization of the RGS proteins, immunohistochemical staining for RGS7, RGS11, G $\beta 5$ and R9AP was performed in Gpr179 $19^{\text {nob5 }}$ fixed retinal sections and the proteins were visualized by confocal microscopy. These data revealed that RGS7 and RGS11 are absent from the DBCs of the Gpr179 ${ }^{\text {nob5 }}$ retina (Fig. 21) and (Orlandi et al., 2012). In addition to RGS7 and RGS11 not localizing to the DBC dendritic tips of the Gpr179 nob5 retina, the RGS membrane anchor R9AP and Gß5 also do not localize to the DBC dendritic tips (Fig. 21). These data suggest that GPR179 either acts as an RGS protein anchor in addition to R9AP, or GPR179 is required to recruit another unidentified RGS anchor to the DBC dendritic tips. These data also suggest that the disruption in DBC signaling in the Gpr179 ${ }^{\text {nob5 }}$ retina may be the result of the RGS complex not localizing correctly. 

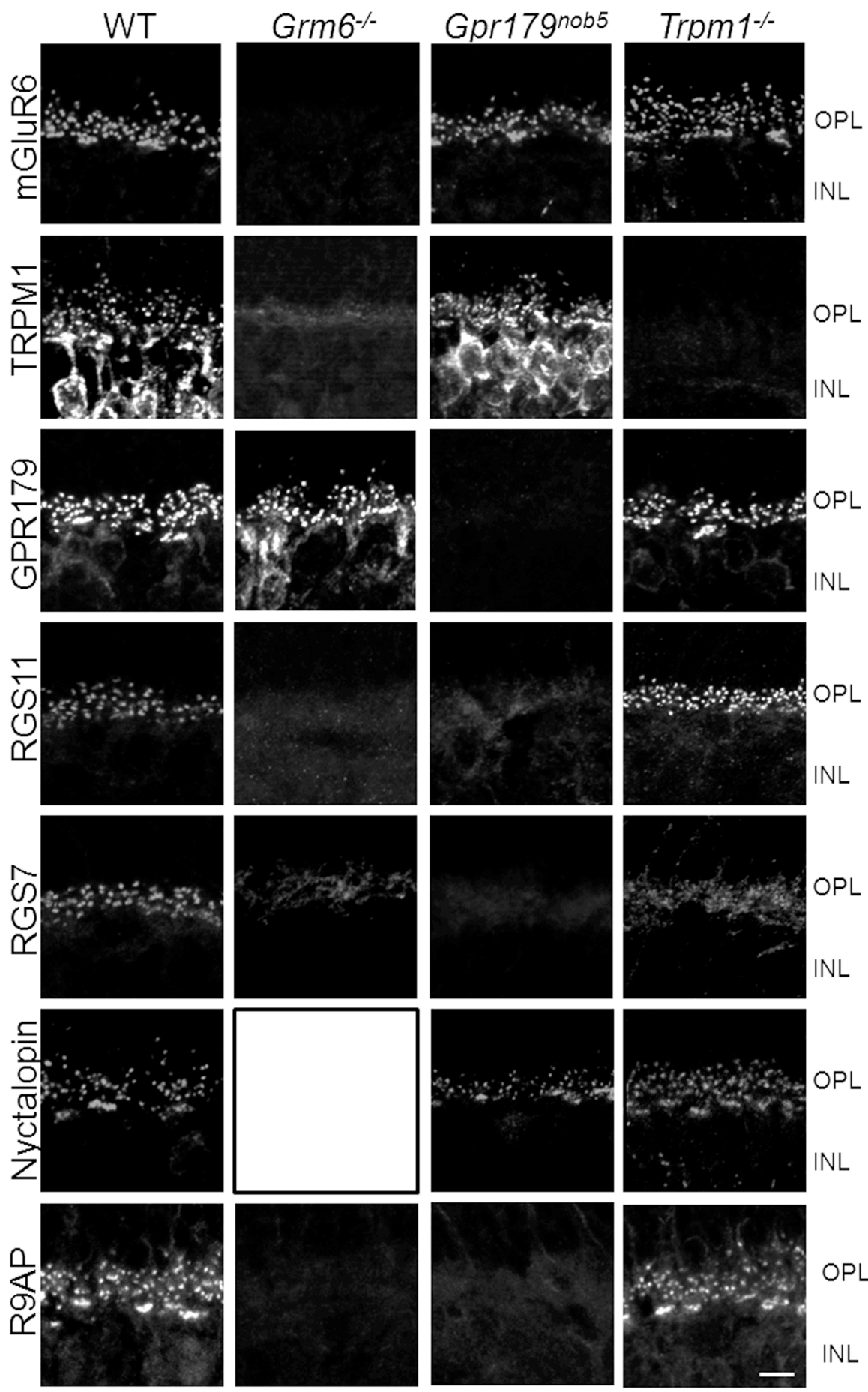

Figure 21: Signlaplex protein expression in mouse models of DBC dysfunction I. Immunohistochemical labeling of signlaplex proteins mGluR6, TRPM1, GPR179, RGS11, RGS7, nyctalopin and R9AP in fixed retinal sections from WT and cCSNB mouse models $\mathrm{Grm6}^{-/-}$, Gpr179 ${ }^{\text {nob5 }}$ and Trpm $1^{-/-}$. The image of TRPM1 staining in the $\mathrm{Grm6}^{-/}$mice is adapted from (Xu et al., 2012). Open box indicates that this particular experiment has not been done. Scale bar is $5 \mu \mathrm{m}$. 


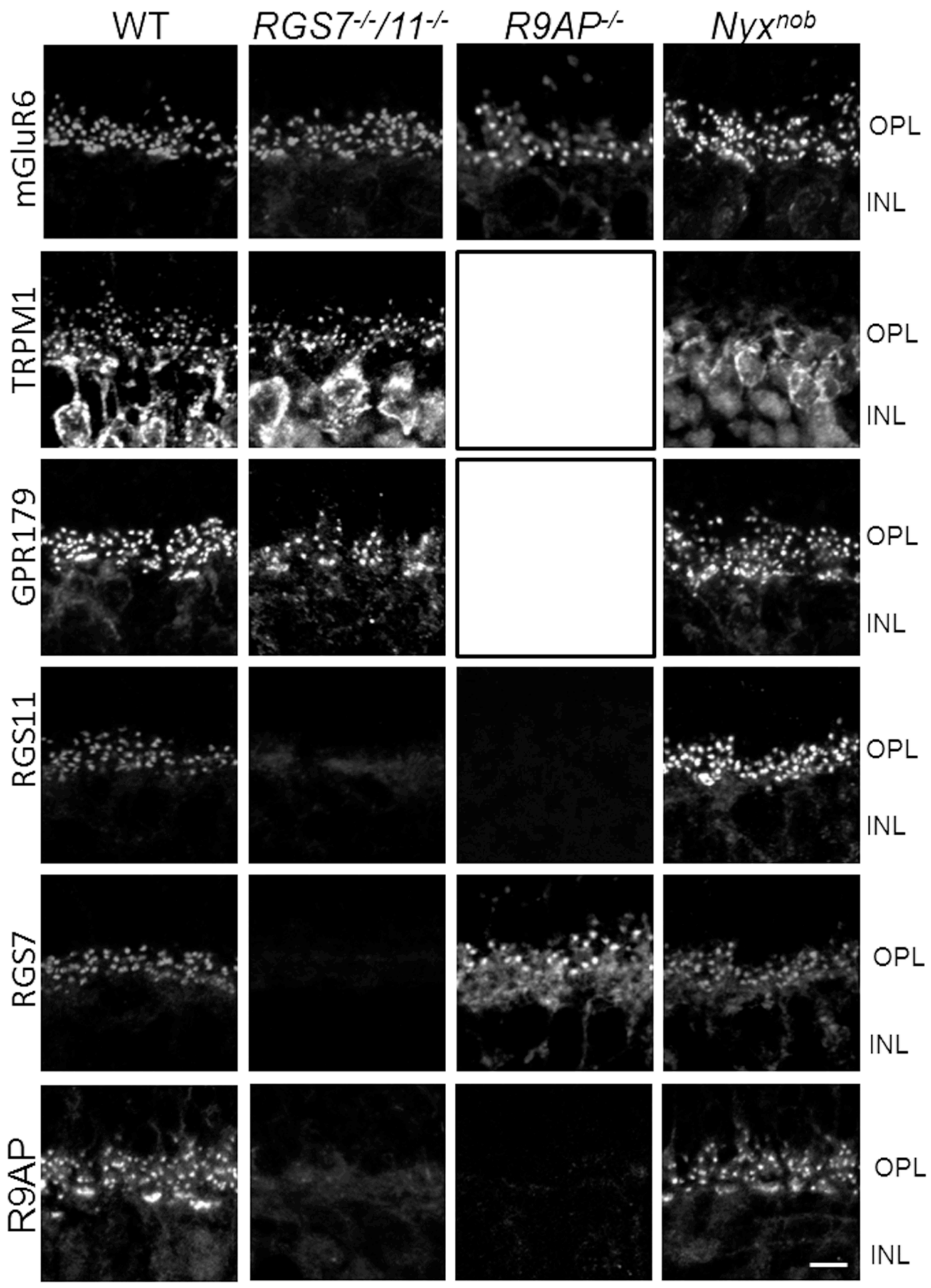

Figure 22: Signlaplex protein expression in mouse models of DBC dysfunction II. Immunohistochemical labeling of signlaplex proteins mGluR6, TRPM1, GPR179, RGS11, RGS7, nyctalopin and R9AP in fixed retinal sections of WT and cCSNB mouse models $R G S 7^{-/} / 11^{-/-}$and $N y x^{n o b}$. Data for $R 9 A P^{-/-}$mice adapted from (Cao et al., 2009). Open box indicates that this particular experiment has not been done. Scale bar is $5 \mu \mathrm{m}$. 


\section{Screening of GPR179 interacting partners using the Membrane Yeast-Two Hybrid}

To determine the role of GPR179 in the DBC signalplex I screened for proteins that may interact with GPR179. My first attempt at identifying interacting partners relied on the membrane yeast two-hybrid (MYTH) system (lyer et al., 2005). The MYTH system requires cloning your genes of interest into two different yeast expression vectors, bait and prey (Fig 23AB). The bait and prey vectors each contain a yeast membrane signal sequence, multiple cloning site and split ubiquitin. In addition, they contain genes involved in the de novo synthesis of leucine or tryptophan, which allows for selection of yeast carrying both plasmids on -leu/-trp selective media. The bait vector expresses your membrane protein of interest fused to the carboxy terminus of a split ubiquitin and the artificial transcription factor LexA-VP16 (Fig. 23C). The prey vector expresses a second TM protein of interest fused to the amino terminus of a split ubiquitin (Fig. 23C). The bait and prey vectors are co-transfected into yeast and plated on -Leu/-Trp selective media to select for yeast containing the bait and prey vectors. The yeast containing both plasmids are then plated on -leu/-trp/-his/-ade selective media. If the proteins interact, the split ubiquitins can dimerize, which recruits the cell's ubiquitin specific proteases to cleave the bait protein and release the transcription factor. The transcription factor will enter the nucleus and promote transcription of genes responsible for de novo synthesis of histidine and adenine in the genetically engineered NMY32 yeast strain allowing growth of yeast that contain interacting bait and prey proteins (Fig. 23C). 
A

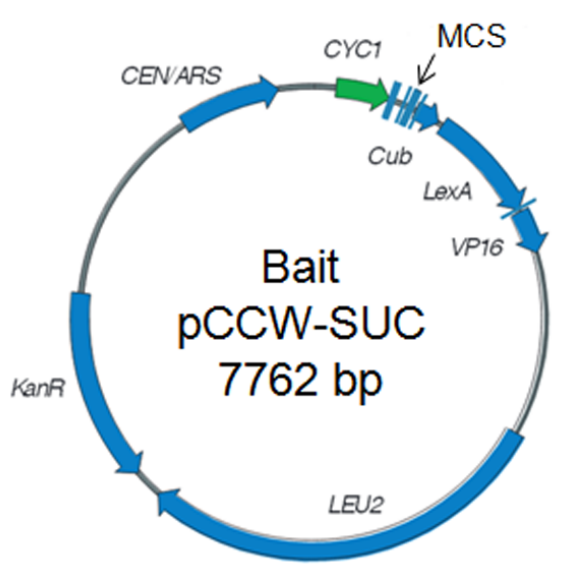

C
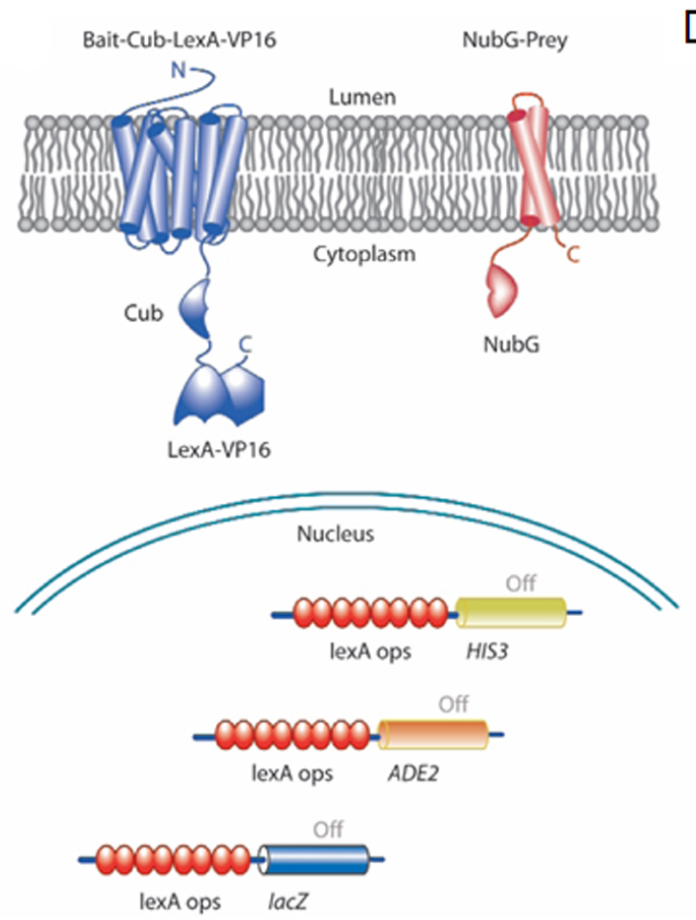

B

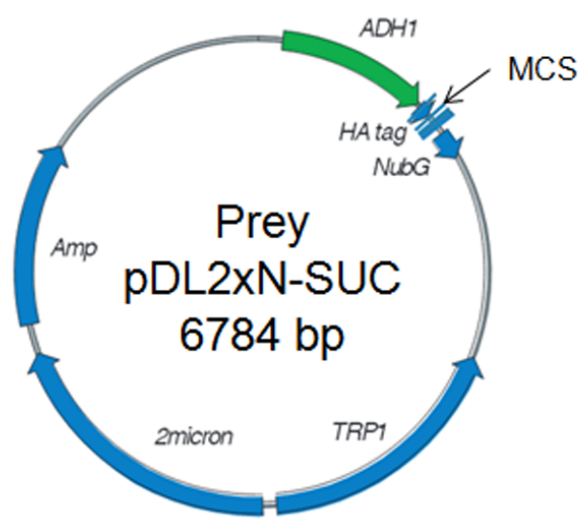

$\mathrm{D}$
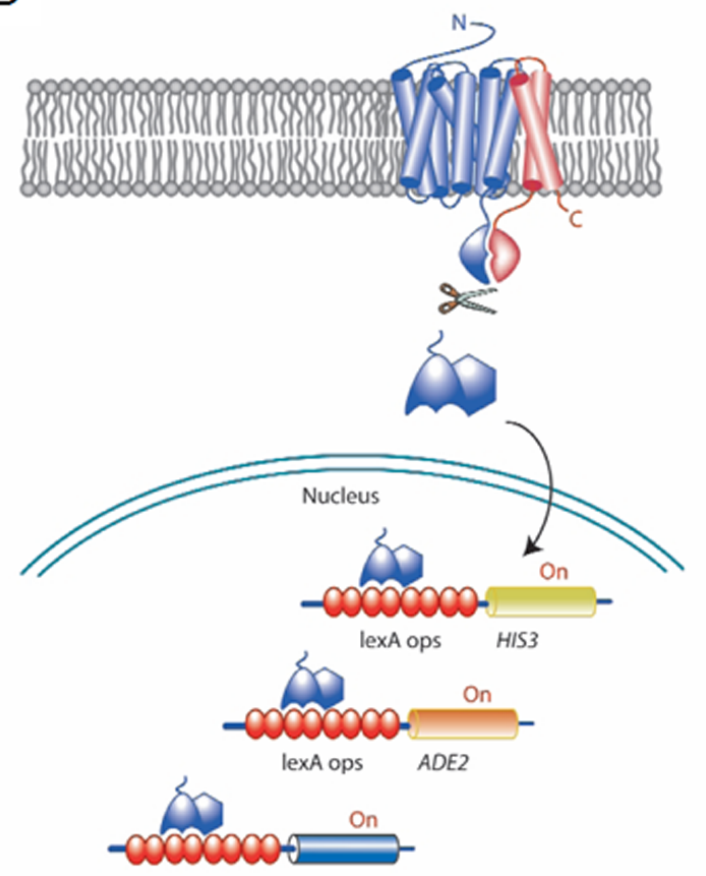

Figure 23: Schematic of MYTH assay. (A) Bait vector contains $\mathrm{CYCl}$ promoter, Cub split ubiquitin and LexA VP16 transcription factor. The bait vector also contains a gene critical for de novo leucine synthesis. (B) The prey vector contains the ADH1 promoter and NubG split ubiquitin. The prey vector also contains a gene critical for de novo synthesis of tryptophan (C) Bait and prey vectors containing genes encoding membrane proteins are co-expressed in yeast. The bait and prey vectors also contain genes critical for de novo amino acid synthesis that allows positive selection. (D) If the bait and prey proteins interact the split ubiquitin molecule dimerizes and proteases cleave the bait protein, releasing the synthetic transcription factor. The transcription factor controls expression of genes needed for de novo synthesis of histidine and adenine in transgenic NMY32 yeast strain, allowing for positive selection of protein interaction. Modified from (lyer et al., 2005). 
To screen for interactions, Gpr179 was cloned into bait and prey vectors. The size of the Gpr179 ORF ( 7.1 kb) and the size of the bait vector ( 7.7 kb) made cloning difficult. Therefore, I cloned truncated genes into bait vectors that would help identify regions critical for interactions with other signalplex proteins. The clone Gpr179 TM1 contains $211-1,938$ bp of the ORF and encodes a protein that contains the 7 TM domains and a truncated 17 amino acid carboxy terminus (Fig. 24A). The clone Gpr179 TM2 contains $82-2,782$ bp of the ORF minus the signal sequence and encodes a protein that contains the entire amino terminus, 7 TM domain and truncated carboxy terminus (Fig. 24B). The clone Gpr179 Cterm contains 1,885-7,101 bp of the ORF and encodes the 1,739 amino acid carboxy terminus (Fig. 24C). The clone Gpr179 contains the entire ORF and encodes the full-length protein (Fig. 24D). Our lab was already in possession of prey vectors containing the genes $G r m 6, \operatorname{Trpm} 1, G \alpha_{o}$ and $G \beta 5$ which I used to screen for interactions with GPR179.

To test for correct expression and stability of the different Gpr179 bait constructs, I cotransfected them with ALG5 or FUR4 positive and negative control vectors. ALG5, localizes to the endoplasmic reticulum (ER) and FUR4 localizes to the plasma membrane (PM). The positive control vector contains the WT amino terminus of the split ubiquitin, Nubl, and will reconstitute with any bait protein in the same cellular compartment allowing yeast to grow on selective media. The negative control prey vector contains a mutated version of the split ubiquitin amino terminus, NubG, and will only reconstitute with $\mathrm{Cub}$ if the bait and prey proteins interact. Co-transfection of Gpr179 TM1 bait vector with the positive control prey vectors yielded positive growth on selective media (Fig. 24A), indicating that the bait protein was being translated in yeast and expressed in the ER and at the PM. Co-transfection of Gpr179 TM1 bait vector with the negative control prey vectors also yielded growth on interaction selective media (Fig. $22 \mathrm{~A})$, indicating that the bait protein was likely self-activating. This likely occurs because 
the protein is being translated but is not able to be processed properly in the yeast, resulting in proteolysis. The proteolysis liberates the transcription factor allowing the yeast to grow on interaction selective media. The other reason for growth on the selective media is that GPR179 TM1 interacts with ALG5 and FUR4. GPR179 TM1 was deemed unsuitable for screening with signalplex proteins.

Co-transfection of the Gpr179 TM2 bait vector with the positive control prey vectors yielded growth on interaction selective media (Fig. 24B), indicating that the bait protein was being translated in yeast and expressed in the ER and at the PM. Cotransfection of Gpr179 TM2 bait vector with the negative control prey vector that localizes to the PM yielded growth on interaction selective media (Fig. 24B). However, co-transfection of Gpr179 TM2 bait and the negative control prey that localizes to the ER did not yield growth on the selective media. This result was puzzling, but due to the growth with one of the negative controls I determined the truncated protein was not suitable for screening for interactions.

Co-transfection of the full length Gpr179 Cterm bait vector with the positive control prey vectors yielded positive growth on interaction selective media (Fig. 24C), indicating that the bait protein was being translated in yeast and expressed in the ER and at the PM. Co-transfection of Gpr179 Cterm bait with both ER and PM negative control prey did not yield growth on interaction selective media (Fig. 24C), indicating that GPR179 Cterm is suitable for screening in the MYTH system.

Co-transfection of the Gpr179 bait vector with the positive control prey vectors yielded positive growth on selective media (Fig. 22D), indicating that the bait protein was being translated in yeast and expressed in the ER and at the PM. Co-transfection of Gpr179 bait vector with the negative control prey vectors did not yield growth on interaction selective media (Fig. 24D), indicating that the full-length protein is suitable for 
screening in the MYTH system. Therefore, I also cloned the full length Gpr179 ORF into a prey vector to test for interaction with the mGluR6 and GPR179 bait proteins. 
A

$$
\text { GPR179 7TM } 1
$$
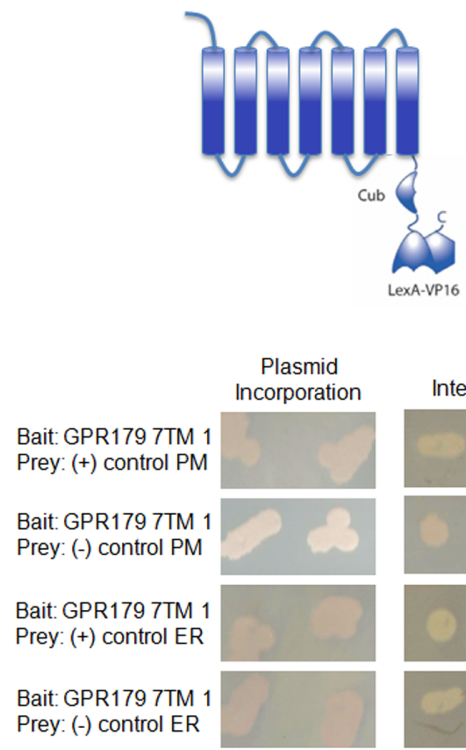

C

GPR179 Cterm

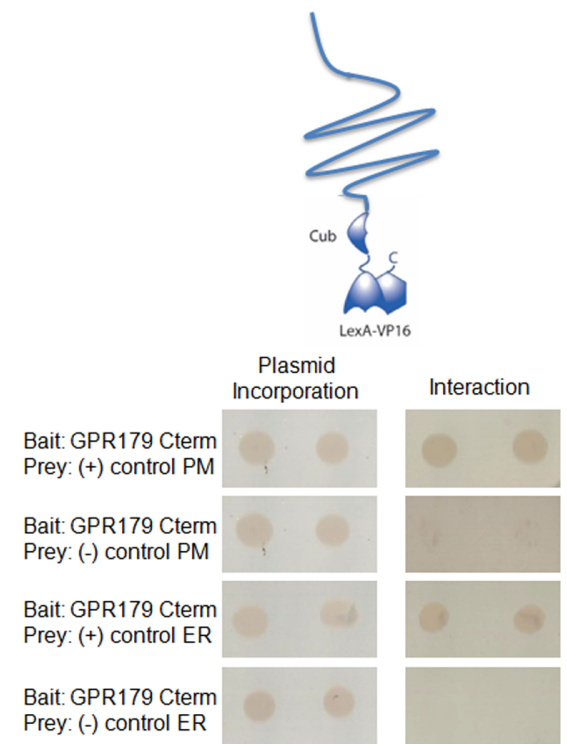

B
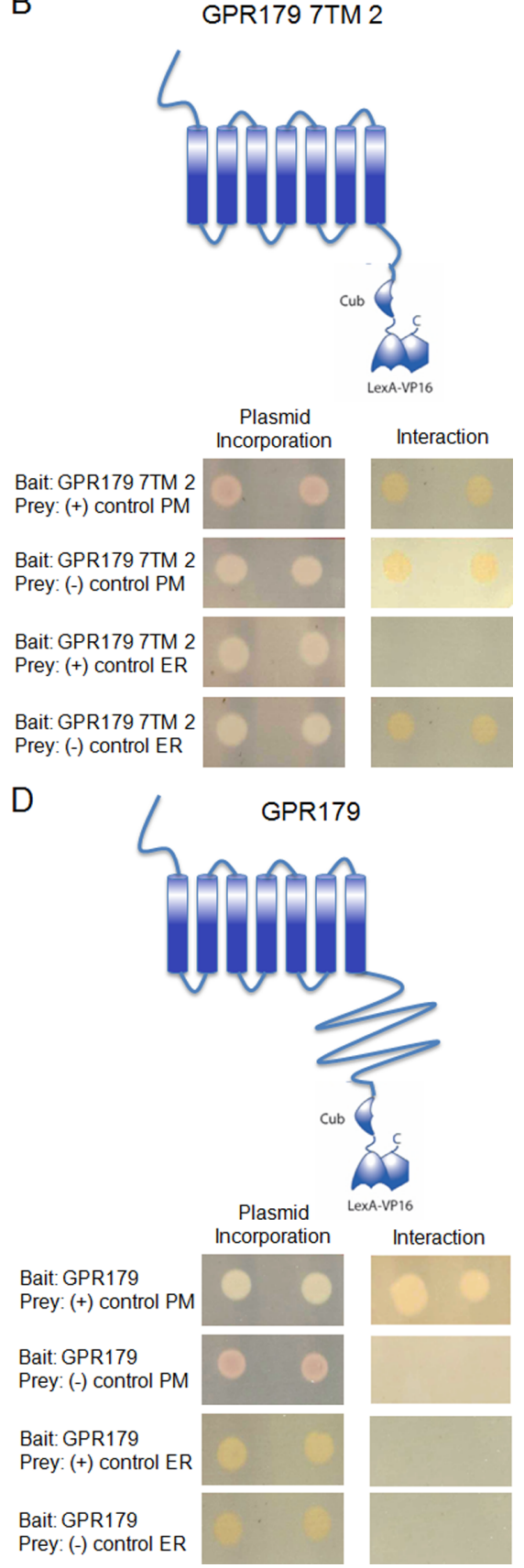

Figure 24: Screening Gpr179 bait vectors for use in MYTH system. (A) The Gpr179 TM1 bait vector encodes a truncated GPR179 protein that does not contain the large cytoplasmic domain. The protein was self-activating when expressed in the MYTH system. (B) The Gpr179 TM2 bait vector encodes a truncated GPR179 protein that contains a small portion of the cytoplasmic domain. The protein was self-activating when expressed in the MYTH system. (C) The Gpr179 Cterm bait vector encodes the cytoplasmic domain of the GPR179 protein and is suitable for screening in the MYTH system. (D) The Gpr179 bait vector encodes the entire GPR179 protein and is suitable for screening in the MYTH system. 
The GPR179 bait protein was co-expressed with TRPM1, Nyctalopin, Gß5, Ga。 or mGluR6 prey proteins (Fig. 25 rows 1-5) and plated on interaction selective media to test for interactions. The bait GPR179 did not test positive for interactions with TRPM1, Nyctalopin, $\mathrm{G} \beta 5, \mathrm{Ga}_{\mathrm{o}}$ or mGluR6 prey proteins. GPR179 was tested for interaction with mGluR6 again by co-expressing the GPR179 prey protein with the mGluR6 bait protein. The yeast did not grow on interaction selective media (Fig. 25 row 6) indicating that the proteins do not interact.

The GPR179 bait protein was tested for self-interaction with the GPR179 prey protein. The screen resulted in positive growth on the interaction selective media indicating that the proteins interact (Fig. 25 row 7). This indicates that GPR179 may be capable of forming homodimers or homooligomers in the yeast system. To test whether this interaction may be occurring through the cytoplasmic protein domain or the TM and extracellular domain, I tested the GPR179 Cterm protein for interaction with the GPR179 prey protein (Fig. 25 row 8). Yeast co-transfected with the Gpr179 Cterm bait and the Gpr179 prey vector did not grow on interaction selective media, indicating that the interaction that allows GPR179 to form homodimers in yeast does not take place on the carboxy terminus of the protein. This means the interaction among GPR179 proteins likely occurs between the 7 TM domains or the extracellular domain. 


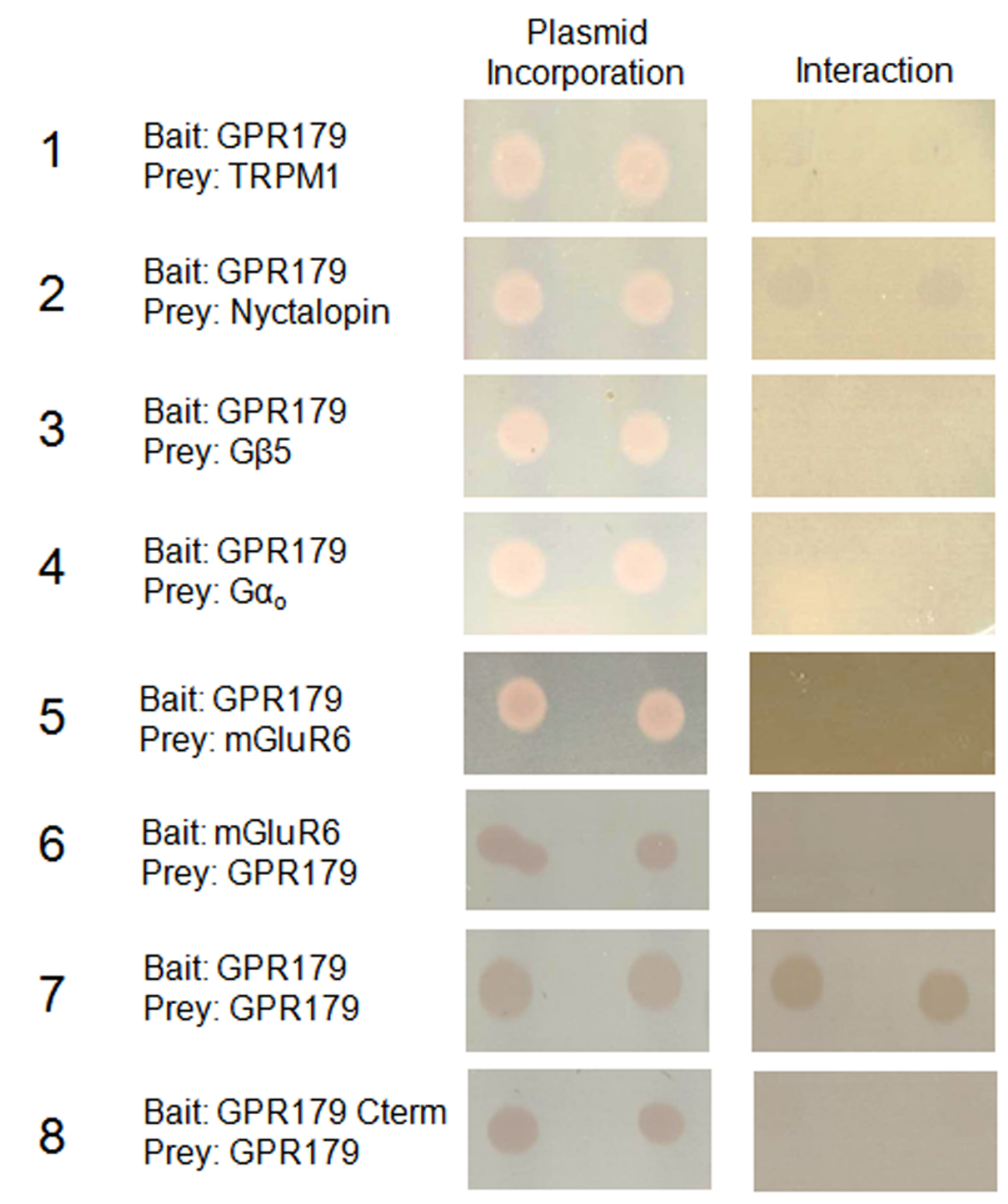

Figure 25: Screening for interactions with the MYTH system. The GPR179 bait protein was tested for interaction with TRPM1, Nyctalopin, G $\beta 5, \alpha_{\circ}$ and mGluR6 bait proteins (Rows 1-5). The mGluR6 prey protein was tested for interaction with GPR179 prey protein (Row 6). Self-interaction was tested with GPR179 bait and prey proteins (Row 7). The self-interaction between GPR179 proteins is not mediated through the cytoplasmic domain of the protein (Row 8). 


\section{Duolink Analyses}

The second method I used to screen for GPR179 interacting partners was the Duolink Proximity Ligation Assay (PLA). The Duolink PLA assay is an antibody ligation assay that produces a positive fluorescent signal only when proteins localize within a critical radius of $\sim 40 \mathrm{~nm}$ of one another. The assay is performed in vitro or in vivo by probing for proteins of interest with primary antibodies produced in different species (Fig. 26A). The primary antibodies are probed for with (+) and (-) PLA probes that consist of secondary antibodies conjugated to an oligonucleotide. The $\left(^{+}\right)$PLA probe oligonucleotides will circularize with the (-) PLA oligonucleotides in the presence of a ligation mixture if the probes are located within $40 \mathrm{~nm}$ of one another. A DNA amplification solution is added to initiate rolling circle amplification of the circularized DNA. The newly synthesized DNA is bound by fluorescent probes, which produce a fluorescent signal that can be visualized by confocal microscopy.

To evaluate the system, I tested whether Grm1, which encodes the protein mGluR1, a Class C GPCR that has been shown to form homodimers in vitro (Doumazane et al., 2011), will interact in this system. Myc and FLAG tagged Grm1 vectors were co-transfected in COS-7 cells and $24 \mathrm{~h}$ post transfection the PLA assay was performed using mouse anti-myc and rabbit anti-FLAG antibodies. Confocal microscopy revealed that the cells co-expressing mGluR1-myc and mGluR1-FLAG produced positive PLA signals, indicating formation of mGluR1 homodimers (Fig. 26B). As a negative control, the assay was performed but application of the primary antibodies was withheld. The negative control shows very little PLA fluorescent signals as expected (Fig. 26C). 
A
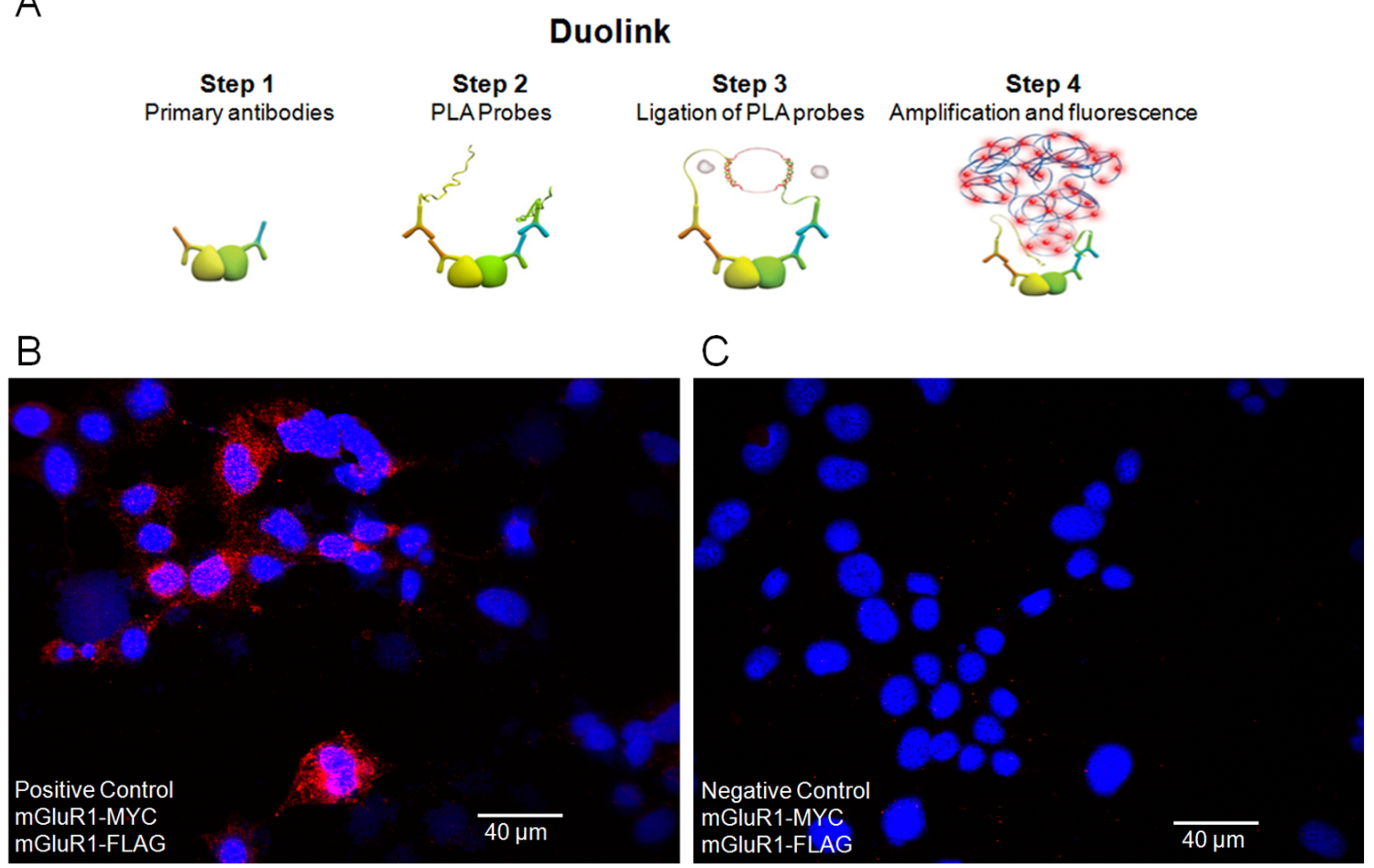

Figure 26: In vitro Duolink PLA assay. (A) Schematic of the Duolink PLA assay. (B) As a positive control, cells were co-transfected with vectors containing Grm1myc and Grm1-FLAG and the Duolink assay was performed. A representative confocal microscopy image shows positive PLA fluorescence is present in cells co-transfected with Grm1-myc and Grm1-FLAG. (C) As a negative control the assay was performed minus the primary antibodies. 
To test for interaction between GPR179 and mGluR6, I co-transfected Gpr179myc and Grm6-FLAG expression vectors into COS-7 cells. The Duolink PLA assay was performed and confocal microscopy revealed cells were co-expressing GPR179-myc and mGluR6-FLAG (Fig. 27A,B,C) and that there is a positive PLA fluorescent signal (Fig. 27D). These data indicated that GPR179 and mGluR6 localize within close proximity to one another and likely interact in this system.

To test for protein interactions between GPR179 and TRPM1, I co-transfected Gpr179-myc and Trpm1-FLAG into Cos-7 cells. After performing the PLA assay confocal microscopy revealed cells were co-expressing GPR179-myc and TRPM1-FLAG (Fig. 27E,F,G) and that a positive PLA fluorescent signal is present (Fig. 27H). These data indicate that GPR179 and TRPM1 localize within close proximity to one another and likely interact in this system. 

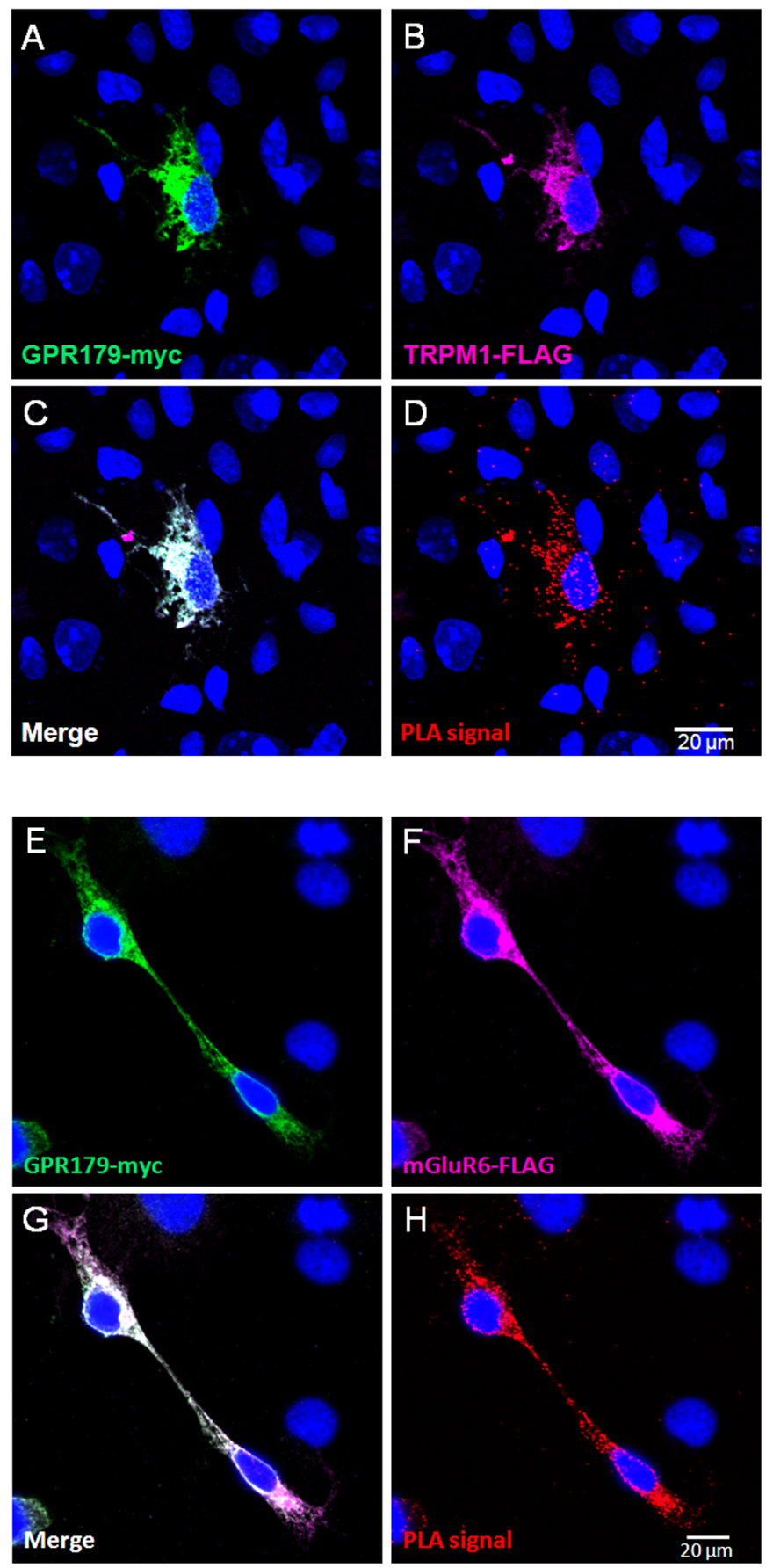

Figure 27: GPR179 interacts with mGluR6 and TRPM1 in vitro. (A-C) GPR179 and TRPM1 are co-expressed in Cos-7 cells and co-localize. (D) PLA fluorescence is present in cells that co-express GPR179 and TRPM1, indicating the proteins interact. (E-g) GPR179 and mGluR6 are co-expressed in Cos-7 cells and colocalize. (H) PLA fluorescence is present in cells that co-express GPR179 and mGluR6, indicating the proteins interact. 
To test if GPR179 and TRPM1 form complexes in vivo, I used the Duolink PLA assay on fixed transverse retinal sections. I used WT retinal sections and antibodies against GPR179 and TRPM1. The PLA assay was performed in conjunction with fluorescent immunostaining for GPR179 and TRPM1 followed by analysis using confocal microscopy. WT retinas labeled with GPR179 and TRPM1 antibodies showed the proteins were localized to the DBC dendritic tips (Fig 28A,B) and there is positive PLA fluorescence in the OPL (Fig. 28C). The PLA fluorescent signal co-localizes with the GPR179 and TRPM1 staining (Fig. 28D), indicating that the signal is specific to regions where both proteins are expressed.

As a negative control, the assay was performed on transverse retinal sections from $N y x^{\text {nob }}$ mice. In the $N y x^{\text {nob }}$ retinas TRPM1 does not localize to the DBC dendritic tips (Fig. 28E) but GPR179 is expressed normally (Fig. 28F), as expected. However, there were no consistent PLA signals present in the OPL that co-localize with GPR179 (Fig. 28G), indicating that a positive PLA signal is dependent on GPR179 and TRPM1 expression at the DBC dendritic tips.

A notable feature of the positive signal from Fig. $28 \mathrm{C}$ is that they are not present at every DBC dendritic tip where GPR179 and TRPM1 are expressed. This suggests that the antibody epitopes for GPR179 and TRPM1 may reside close to the critical distance for the assay, which would lower the efficiency of a positive reaction. The protein dense environment where GPR179 and TRPM1 reside could also decrease the Duolink assay efficiency, accounting for puncta expressing both proteins but lacking a positive signal. 

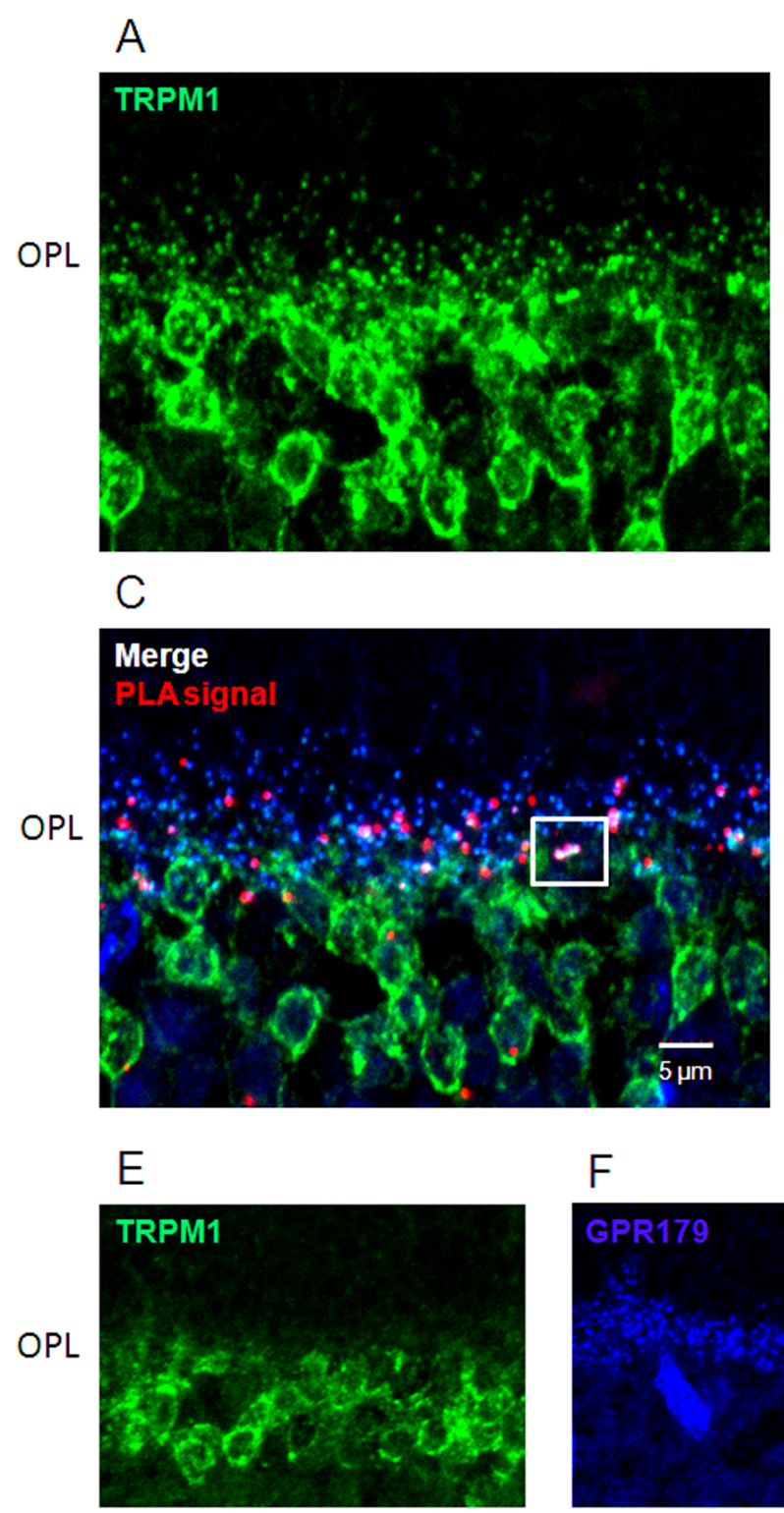

$\mathrm{B}$

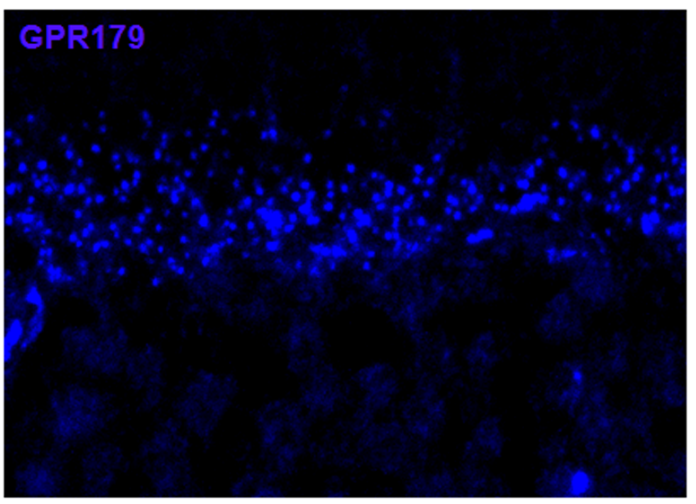

D
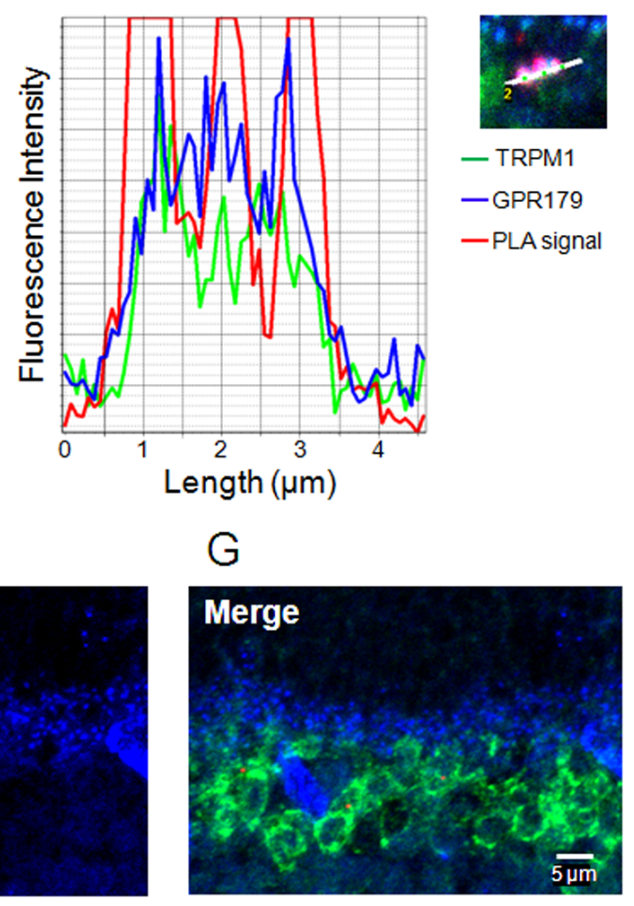

F

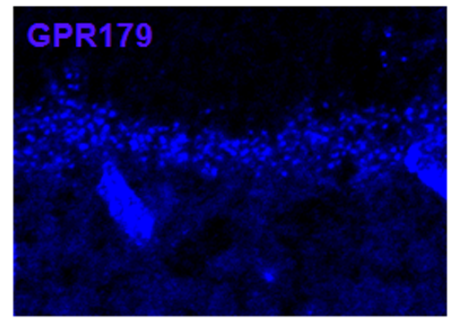

Figure 28: TRPM1 and GPR179 localize within the Duolink critical radius. (A) WT retina slices labeled with antibodies against GPR179 (blue) and TRPM1 (green) and PLA assay was performed. Red puncta indicate positive interactions. (B) PLA assay was performed in $n y x^{\text {nob }}$ retinas (negative control) which lack TRPM1 expression at the DBC dendritic tips. (C) A $4.5 \mu \mathrm{m}$ line was drawn through a cone pedicle in (A) and fluorescence was plotted across the line. GPR179, TRPM1 and the PLA flurescence overlap, indicating the PLA fluorescent signal is specific to puncta expressing GPR179 and TRPM1. 


\section{Discussion}

The immunohistochemistry data shows GPR179 is critical for the localization of RGS7, RGS11, Gß5 and R9AP (Fig. 21 and Orlandi et al. (2012)). We also showed that GPR179 physically interacts with the RGS7/G $\beta 5$ and RGS11/G $\beta 5$ protein complexes (Orlandi et al., 2012). These data indicate that GPR179 may act as a scaffolding protein for both RGS7 and RGS11 in the DBCs and that the interaction is dependent on the expression of Gß5. We know that the RGS proteins are critical to setting the light response in the DBCs (Cao et al., 2012). One aspect of my data that has not been studied further is the absence of the membrane anchor R9AP in the Gpr $179^{\text {nob5 }}$ DBCs. It is unknown whether R9AP interacts directly with GPR179. Further, it appears that RGS7/Gß5 or RGS11/Gß5 binding with R9AP is required for R9AP localization at the DBC dendritic tips because R9AP is absent in $R G S 7^{-/} / R G S 11^{-/}$retinas (Fig. 22). It will also be important to determine whether R7BP, another RGS anchor expressed in the DBCs, is affected by GPR179 expression. It seems likely that GPR179 may act as a hub to recruit a large number of RGS proteins to the DBC dendritic tips so that a large number of GAP proteins are localized to the signalplex to set the speed of the BC light response. The large cytoplasmic tail on GPR179 is an ideal domain to act as a hub. Large, unstructured protein domains are known to act as protein scaffolds (Dyson and Wright, 2005; Coletta et al., 2010).

It was previously reported that RGS7, RGS11, G 35 and TRPM1 expression were decreased or absent in the $\mathrm{Grm6}^{-/}$retina (Morgans et al., 2007; Cao et al., 2009; Xu et al., 2012). Immunolabeling GPR179 in the $\mathrm{Grm6}^{-/-}$retina reveals that GPR179 is properly localized to the dendritic tips of the DBCs. The dependence of TRPM1 localization on mGluR6 is controversial. Cao et al. (2011) reported TRPM1 was absent from the OPL in Grm6 $6^{\text {nob3 }}$ retinas, a Grm6 spontaneous mutant, whereas Xu et al. (2012) found it was present but greatly decreased. Other proteins absent from the DBC 
dendritic tips in $\mathrm{Grm6}^{-/}$retinas include Gß5, RGS7, RGS11 and R9AP (Fig. 21) and (Morgans et al., 2007; Xu et al., 2012). These data indicate that Gß5, RGS7, RGS11 and R9AP localization is dependent on mGluR6 expression, whereas GPR179 is not (Fig. 21). When taken together with the data from the $G p r 179^{\text {nob5 }}$ retinas, which also lose localization of Gß5, RGS7, RGS11 and R9AP but maintain mGluR6 localization, the mechanism by which the proteins do not localize in the $\mathrm{Grm}^{-/}$retinas is unclear. The creation of a dominant negative mGluR6 mouse in which it traffics to the DBC dendritic tips would help sort out whether the loss of proteins is because they are physically dependent on mGluR6 for trafficking or if the signalplex is downregulated by another mechanism.

The MYTH assay to test for protein interactions between GPR179 and other DBC signalplex proteins did not produce positive data that GPR179 interacts with other signalplex proteins. This could be due to the fact that yeast and BCs are such different systems that proteins may not behave the same way in each system. In the DBCs there are likely additional proteins that traffic and add posttranslational modifications to DBC signalplex proteins. This facilitates organization and trafficking of signalplex proteins at the dendritic tips in an ordered complex. In contrast, the MYTH system requires two exogenous mammalian fusion proteins to be expressed in a foreign system. The yeast may not be able to add the correct post-translational modifications to the exogenous proteins. They also lack expression of the rest of the signalplex proteins, which may facilitate protein localization.

The in vitro Duolink assay suggested that GPR179 forms complexes with mGluR6 and TRPM1. The downside to the assay is that the positive signal could be due to the overexpression of two membrane proteins, which could cause false positive signals because the cell is being overloaded with proteins, which may force proteins to localize within the Duolink distance. I think these experiments should be performed 
under more stringent conditions by driving gene expression using a weak gene promoter to reduce false positives due to high protein concentration.

The in vivo Duolink assay suggests that GPR179 and TRPM1 are a part of the same protein complex in the DBCs. Therefore, I performed the assay on retinal sections to determine if GPR179 is in the same complex as mGluR6 and nyctalopin. I did not get positive interaction data for these experiments, but I think the reason for this is not because GPR179 does not localize in close proximity with these proteins. Instead, I think the negative results reflect the shortcomings of the assay. If the protein epitopes are on opposite sides of the membrane, as they are with mGluR6 and GPR179, then the assay efficiency is likely decreased dramatically. If the antibody epitopes are on distal regions of the proteins they may be too far apart for the PLA probes to ligate. Finally, the dense protein matrix at the DBC signalplex may inhibit the assay, accounting for the reason the assay was more robust in the in vitro system.

To further examine the positive interaction data between GPR179/TRPM1 and GPR179/mGluR6 Dr. Nazarul Hasan, a postdoc in the lab, performed immunoprecipitation (IP) experiments in parallel. Using antibodies against GPR179, the GPR179 protein complex was immunoprecipitated from retinal lysates and the precipitate was examined by mass spectrometry. These experiments showed that TRPM1 interacts with GPR179 (Ray et al., 2013 and Appendix III). Performing the IP with antibodies against TRPM1 identified GPR179 in the proteins that were precipitated. To validate the mass spectrometry identification that GPR179 and TRPM1 were in the same protein complex Co-IP experiments were performed in mammalian cell lines. The Co-IP experiments showed positive interaction between GPR179 and TRPM1 (Ray et al., 2013 and Appendix III). IP experiments from retinal lystates also suggested that TRPM1 and GPR179 directly interact (Ray et al., 2013 and Appendix III). 
The self-interaction between GPR179 bait and prey vectors should be further studied. It is well accepted that many GPCRs are known to form homo and heterodimers and even higher order oligomers (Bouvier, 2001; Milligan, 2006; Filizola, 2010). The ability of GPR179 to form dimers or oligomers would enable it to be the focal point of the signalplex protein structure, especially if it is found to interact with more DBC signaling proteins.

The interaction between GPR179 and TRPM1 likely enhances the sensitivity of the glutamate signaling cascade by bringing the RGS machinery in close proximity to the TRPM1 channel. This enables the TRPM1 channel to quickly respond to changes in glutamate release from the photoreceptors because the RGS proteins continuously hydrolyze $\mathrm{Ga}_{\circ} \mathrm{GTP}$ to $\mathrm{Ga} a_{\circ} \mathrm{GDP}$, which presumably inhibits $\mathrm{G}$ protein closure of the TRPM1 channel. Our collaborator Neal Peachey observed a small ERG b-wave response in the Gpr179 ${ }^{\text {nob5 }}$ mouse that was not present in other cCSNB mouse models (Ray et al., 2013 and Appendix III). When the ERG flash duration was increased, the bwave response increased in the Gpr179 ${ }^{\text {nob5 }}$ mouse but the $\operatorname{Trpm}^{-/}$did not have a bwave. These observations were the catalyst for performing single cell recording experiments that identified a role for GPR179 beyond anchoring RGS proteins.

The immunohistochemistry revealed that mGluR6 and TRPM1 localized correctly in the Gpr179 nob5 DBCs and the ERG suggested these mice had a small b-wave. Therefore, we asked whether mGluR6 could gate the TRPM1 channel in the Gpr179 nob5 retina. To answer that question we used single cell recordings from rod BCs and found that CPPG, an mGluR6 antagonist, could illicit a response in the Gpr179 $9^{\text {nob5 }}$ rod BCs that was significantly decreased from WT but significantly greater than the Trpm1 ${ }^{-/}$rod BC response (Ray et al., 2013 and Appendix III). We followed that experiment up by directly testing the gating of the TRPM1 channel in the Gpr179 ${ }^{\text {nob5 }}$ rod BCs using single cell recordings and the drug Capsaicin, a TRPM1 channel modulator, to gate the channel 
open. We found that in the Gpr179 ${ }^{\text {nob5 }}$ rod BCs there was a significantly decreased capsaicin response compared to WT. These data suggested that the TRPM1 channel is not as sensitive to gating in the absence of GPR179.

We hypothesized that the interaction between GPR179 and TRPM1 enhanced the TRPM1 sensitivity to gating by changing the state of the channel in the membrane through physical interaction. To determine if GPR179 was required for the high sensitivity of the TRPM1 channel to capsaicin we performed the capsaicin experiments in the $R G S 7^{-/ /} / R G S 11^{-/}$rod BCs, which do not express the RGS protein complex but retain all other signalplex components (Fig. 22). We found that the $R G S 7^{-/} / R G S 11^{-/-}$rod BCs had a capsaicin response similar to WT and significantly greater than the response in the Gpr179 ${ }^{\text {nob5 }}$ rods BCs (Ray et al., 2013 and Appendix III). These data suggested that GPR179 is required to enhance the sensitivity of the TRPM1 channel to gating.

Channel variance can be measured when the retinal sections are bathed in LAP4 and indicate the state of the TRPM1 channel when the glutamate signaling cascade is maximally active. High variance indicates the channels are in a state of constant opening and closure, whereas low variance indicates the channels are either maximally open or maximally closed. In WT retinal sections variance should be low under L-AP4 due to $G$ protein closure of the TRPM1 channel. We observed significantly lower channel variance in Gpr179 ${ }^{\text {nob5 }}$ rod BCs compared to WT. The channel variance in the Gpr179 ${ }^{\text {nob5 }}$ rod BCs was similar to the variance in Trpm $1^{-/}$rod BCs. These data suggest that in WT rod BCs the TRPM1 channel is constantly opening and closing in response to

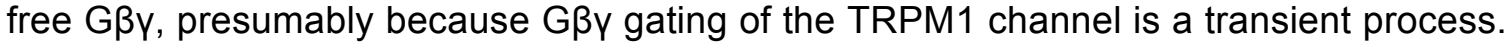
In the Gpr179 ${ }^{\text {nob5 }}$ rod BCs the variance is decreased similar to the $R G S 7^{-/ /} / R G S 11^{-/}$rod BCs (Ray et al., 2013 and Appendix III). We attribute these results to the mislocalized or absent RGS proteins in the Gpr179 ${ }^{\text {nob5 }}$ and $R G S 7^{-/} / R G S 11^{-/}$rod BCs that allows a build up of active G proteins shifting the equilibrium of TRPM1 channels to more closed. 
We were able to show that GPR179 plays a structural role in the DBCs by recruiting the RGS proteins to the signalplex. We also found that GPR179 directly interacts with TRPM1 and that this interaction is critical to the gating sensitivity of the TRPM1 channel. The disruption in glutamate signaling in the Gpr179 ${ }^{\text {nob5 }}$ rod BCs appears to be the compound result of a loss of RGS proteins and decreased TRPM1 channel sensitivity. A recent publication also reported that GPR179 physically interacts with mGluR6 in the DBCs (Orlandi et al., 2013). Taken together, these data suggest that GPR179 is a hub for DBC signalplex components. Although GPR179 is not critical for the localization of mGluR6 and TRPM1 to the DBC dendritic tips, it may play an important role in recruiting these proteins into a larger oligomeric structure.

There is still disagreement about whether $\mathrm{Ga}_{\circ}$ or $\mathrm{G} \beta y$ is responsible for gating the TRPM1 channel (Koike et al., 2010a; Koike et al., 2010b; Shen et al., 2012) and there is almost nothing known about the detailed mechanism of gating. Whole cell patch clamp experiments in rod BCs suggest that introduction of $\mathrm{G} \beta \mathrm{y}$ into the rod $\mathrm{BCs}$ via the patch pipette closes the TRPM1 channel, whereas $\mathrm{Ga}_{0}$ does not (Shen et al., 2012). These data suggest that the rod BCs respond to glutamate by mGluR6 binding glutamate and activating the heterotrimerc $G$ proteins through $\mathrm{Ga}_{\circ} \mathrm{GDP}$ to $\mathrm{Ga} \alpha_{0} \mathrm{GTP}$ exchange, which subsequently causes $\mathrm{Ga}_{\circ} \mathrm{GTP}$ to release from the $\mathrm{G} \beta \mathrm{y}$ complex. The GßY dimer binds and directly closes the TRPM1 channel or closes the TRPM channel through an effector protein.

\section{Methods}

\section{Antibodies}

The following primary antibodies and their concentrations were used: sheep antiGPR179 (peptide KVQEETPGEDLDRPVLQKR), 1:2,000 (Peachey et al., 2012b); mouse monoclonal anti-ctbp2/Ribeye (BD Bioscience), 1:1,000; guinea pig anti-mGluR6 1:1,000 (Koike et al., 2010b); sheep anti-TRPM1 1:1,000 (Cao et al., 2011); rabbit anti- 
GFP (MBL), 1:800 and Rhodamine Peanut Aglutinin (PNA) conjugate 566 (Vector Labs), 1:1,000; goat anti-Gß5 (peptide MATDGLHENETLASLKC), 1:1,000 (Morgans et al., 2007); goat anti-R9AP 1:1,000 (Hu and Wensel, 2002); mouse anti-DIG HRP, 1:1,000 (Perkin Elmer); mouse anti-GFP, 1:1,000 (Molecular Probes); sheep anti-RGS11 (peptide CSPALQSTPREPAATSSPEGADGE), 1:1,000 (Martemyanov et al., 2005); sheep anti-RGS7 1:1,000 (Morgans et al., 2007); mouse anti-RIBEYE 1:1,000 (BD Transduction Labs); rabbit anti-PKC 1:1,000 (Sigma Aldrich).

Secondary antibodies (Invitrogen, 1:1,000) appropriate to each primary antibody included: donkey anti-sheep Alexa-488, donkey anti-rabbit Alexa-680, donkey anti-rabbit Alexa-546, donkey anti-mouse Alexa-647 and donkey anti-guinea pig Cy3 (Millipore, $1: 1,000)$. In lieu of an antibody specific to nyctalopin, we used $T g(G a b r r 1-Y F P / n y x)^{R g g 1}$ transgenic mice that express a yellow fluorescent protein (YFP) tagged nyctalopin (Gregg et al., 2007). They are labeled as WT in the figures.

\section{Immunohistochemistry}

Mice were euthanized with $\mathrm{CO}_{2}$ followed by cervical dislocation. Their eyes were enucleated and the lens was removed. Eyecups were washed in phosphate buffered saline (PBS) then fixed for $30 \mathrm{~min}$ in a $4 \%$ formaldehyde PBS solution ( $\mathrm{pH} 7.4)$. Eyecups were washed 3 times with PBS then cryoprotected in increasing concentrations of sucrose in PBS $(10 \%, 15 \%$ for $1 \mathrm{~h}$ each and $30 \%$ overnight). Eyecups were embedded in 2:1 OCT/ $20 \%$ sucrose PBS solution frozen in a liquid nitrogen cooled bath of isopentane. Eyecups were sectioned $(18 \mu \mathrm{m})$ using a Leica 1850 cryostat. Sections were mounted on glass slides (SuperFrost) and allowed to dry for 10 mintues at room temperature before being stored at $-80^{\circ} \mathrm{C}$. Sections were air dried at $37^{\circ} \mathrm{C}$ for $15 \mathrm{~min}$ and washed with PBS and PBS containing $0.05 \%$ Triton X-100 (PBX) for 5 min each, then blocked in PBX plus 5\% normal donkey serum blocking solution for $1 \mathrm{~h}$. Sections were incubated overnight at room temperature in the presence of the primary antibody diluted 
in blocking solution, then washed 3 times for 10 min each with PBX followed by incubation in secondary antibody in blocking solution for $1 \mathrm{~h}$ at room temperature. Sections were washed $2 \times 10$ min in PBX and 1 X PBS. Slides were cover-slipped using Immu-Mount (Thermo Scientific) and slides were imaged using an Olympus FV1000 confocal microscope. Images were universally adjusted for brightness using Photoshop.

\section{In-Fusion HD Cloning}

PCR fragments were amplified using primers designed to have 14-18 bp 5' overhangs that share homology with the cut site on a linearized vector. PCR fragments were treated with Cloning Enhancer (Clontech) and incubated at $37^{\circ} \mathrm{C}$ for $15 \mathrm{~min}$ then at $80^{\circ} \mathrm{C}$ for 15 min on a thermal cycler. Linearized vector, treated PCR fragment and water was added to $5 \mathrm{X}$ In-Fusion HD Enzyme Premix according to the manufacturers protocol (Clontech). The reaction was mixed by pipetting and incubated for $15 \mathrm{~min}$ at $50^{\circ} \mathrm{C}$ on a thermal cycler. The reaction mix was placed on ice until transformation or stored at $20^{\circ} \mathrm{C}$.

\section{Plasmid Isolation}

Plasmid purification was performed using the Wizard Plus SV Minipreps DNA Purification System (Promega) according to the manufacturers protocol. Single colonies from transformed bacteria were selected from agarose plates using a sterile toothpicks and used to inoculate $2 \mathrm{~mL}$ of Circlegrow media supplemented with $100 \mu \mathrm{g} / \mathrm{mL}$ kanamycin. The culture was grown overnight at $37^{\circ} \mathrm{C}$ while shaking at $225 \mathrm{RPM}$. The bacterial cells were transferred to a $2 \mathrm{~mL}$ centrifuge tube and pelleted by centrifugation at max speed for 5 minutes to pellet cells. The supernatant was discarded and the cells were resupsended by vortexing in $250 \mu \mathrm{l}$ of Cell Resuspension Solution. $250 \mu \mathrm{l}$ of Cell Lysis Solution and $10 \mu \mathrm{l}$ Alkaline Protease Solution was added, and mixed by inverting tubes several times, then incubated at room temperature for $5 \mathrm{~min}$. $350 \mu \mathrm{l}$ of Neutralization Solution then was added and mixed by inverting several times. Tubes 
were centrifuged at $12,000 \times \mathrm{g}$ for $10 \mathrm{~min}$ to pellet cellular debris and the supernatant was decanted into a spin column. The spin column was placed into a collection tube and centrifuged for $1 \mathrm{~min}$ at $12,000 \times \mathrm{g}$. The flow through was discarded and the plasmid bound to the column was washed 2 times by adding $500 \mu \mathrm{l}$ of Column Wash Solution followed by centrifugation for $1 \mathrm{~min}$ at $12,000 \times \mathrm{g}$. The spin column was transferred to a clean microcentrifuge tube and $100 \mu \mathrm{l}$ of sterile water was added to the column to elute plasmid DNA. The water was incubated on the column for $1 \mathrm{~min}$ at room temperature then centrifuged for $1 \mathrm{~min}$ at $12,000 \times \mathrm{g}$ to collect DNA. DNA was stored at $-20^{\circ} \mathrm{C}$.

\section{Cell culture, transfection and immunocytochemistry}

COS-7 (African green monkey kidney) cells were cultured in high glucose DMEM supplemented with $10 \%$ fetal bovine serum, $2 \mathrm{mM}$ L-glutamine, $50 \mathrm{IU} / \mathrm{ml}$ penicillin and $50 \mu \mathrm{g} / \mathrm{ml}$ streptomycin. $25 \mathrm{~h}$ prior to transfection, cells were seeded on $1 \mathrm{~cm}^{2}$ chamber slides (Lab-Tek). Expression plasmids were transfected into COS-7 cells using Lipofectamine 2000 (Invitrogen) according to the manufacturer's instructions. $24 \mathrm{~h}$ after transfection, cells were washed in PBS and fixed in ice-cold methanol for 5 min then icecold methanol/acetone (50:50) for $5 \mathrm{~min}$. Cells were washed in PBS and blocked for $1 \mathrm{~h}$ in blocking solution (PBS containing $0.01 \%$ Triton $\mathrm{X}-100$ and $5 \%$ normal donkey serum). Antibodies were diluted in blocking solution and incubated on cells overnight at $4^{\circ} \mathrm{C}$, then washed with PBS. Fluorescent secondary antibodies were diluted 1:1,000 and added to slides. Cells were incubated for $1 \mathrm{~h}$ with secondary antibodies at room temperature then washed with PBS. Slides were mounted with coverslips using Vectashield mounting medium with DAPI (Vector Laboratories). Slides were imaged with an FV-1000 confocal microscope.

\section{Duolink}


Duolink assay was performed as described in the Duolink II Fluorescence user manual (OLink Bioscience). Retina sections were prepared for immunohistochemistry as previously described. Sections were air dried at $37^{\circ} \mathrm{C}$ for $15 \mathrm{~min}$ and washed with PBS and PBS containing $0.05 \%$ Triton X-100 (PBX) for 5 min each and then blocked in PBX containing 5\% normal donkey serum for $1 \mathrm{~h}$. Primary antibodies were diluted in blocking serum and incubated on sections overnight at room temperature. Slides were washed $3 \times 10$ min in PBX. In the meantime, PLA probes specific to the primary antibodies were mixed 1:5 in blocking solution and incubated at room temperature for 20 min. After the slides were washed, the solution containing the PLA probes was applied and incubated at $37^{\circ} \mathrm{C}$ in a humidity chamber for $1 \mathrm{~h}$. Slides were washed $2 \times 5 \mathrm{~min}$ in PBX. In the meantime, Ligation stock was diluted 1:5 in PCR grade water and mixed. Just before adding to slides, Ligase was added to the Ligation mixture at a 1:40 dilution, mixed gently. Slides were incubated at $37^{\circ} \mathrm{C}$ in a humidity chamber for $100 \mathrm{~min}$. Slides were washed $2 \times 10 \mathrm{~min}$ in $\mathrm{PBX}$ and $1 \times 10 \mathrm{~min}$ in PBS. For fluorescent immunostaining, fluorescent secondary antibodies were diluted in blocking solution and incubated for $1 \mathrm{~h}$ at room temperature. Slides were washed $2 \times 10 \mathrm{~min}$ in PBX and $1 \mathrm{X}$ 10 min in PBS. Slides were mounted with glass coverslips using Duolink Mounting Medium containing DAPI then visualized on a confocal microscope.

\section{Membrane yeast-two hybrid}

Yeast were grown overnight in $10 \mathrm{ml}$ YPD media (Clontech) to $A_{600} 0.5$ to 1.0 . Yeast were pelleted for $5 \mathrm{~min}$ at $1500 \times \mathrm{g}$. The pellet was washed with $\mathrm{dd}_{2} \mathrm{O}$ and centrifuged again for $5 \mathrm{~min}$ at $1500 \times \mathrm{g}$. The pellet was re-suspended in (100 $\mu \mathrm{l}$ or $200 \mathrm{I}$ per tube) of TE buffer (10mM Tris- $\mathrm{HCl}, 0.1 \mathrm{mM}$ EDTA) pH 7.5 containing $0.1 \mathrm{M}$ lithium acetate (LiAC). Yeast solution was transfered to a microfuge tube (200ul per tube) and $1 \mathrm{ug}$ of bait and prey plasmid DNA was added in addition to $5 \mu \mathrm{l}(50 \mu \mathrm{g})$ carrier DNA (salmon sperm, denatured for $5 \mathrm{~min}$ at $99^{\circ} \mathrm{C}$ put on ice, denatured again then place on 
ice). To the tube, $300 \mu \mathrm{l} 40 \%$ polyethylene glycol $(250 \mu \mathrm{l} 50 \%)$ in TE containing $0.1 \mathrm{M}$ LiAC was added and vortexed to mix well. Solution was incubated for 30 min at $30^{\circ} \mathrm{C}$, then DMSO added to $10 \% \mathrm{v} / \mathrm{v}$. Cells were heat shocked for $10 \mathrm{~min}$ at $42^{\circ} \mathrm{C}$ then centrifuged for $15 \mathrm{sec}$ at $10,000 \mathrm{xg}$. The supernatant was removed and the cells were resuspended in $100 \mu \mathrm{l}$ of $\mathrm{ddH}_{2} \mathrm{O}$ and plated on selective plates (Minimal SD agar, -His/Ade selective DO supplement, Clontech). Plates were incubated at $30^{\circ} \mathrm{C}$ for $2-3$ days. After 2-3 days, yeast colonies were selected with a sterile pipette tip and re-suspended in $20 \mu \mathrm{l}$ of $\mathrm{ddH}_{2} 0$. Yeast were spotted on interaction selective plates (Minimal SD agar, His/-Ade/-Leu/-Trp selective DO supplement, Clontech) and incubated at $30^{\circ} \mathrm{C}$ for $2-3$ days. 


\section{CHAPTER IV: LRIT3 IS REQUIRED FOR NORMAL DBC FUNCTION}

\section{Aim 3: Determine the mechanism by which LRIT3 contributes to glutamate signaling in the DBCs}

Rationale: Zeitz et al. (2013b) reported that LRIT3, a gene of previously unknown function, was critical to the glutamate signaling cascade in the DBCs. Two human patients with cCSNB of a previously unknown cause were found to have mutations in the LRIT3 (Zeitz et al., 2013b). The mechanism by which LRIT3 contributes to the glutamate signaling cascade remains unknown. To determine the role LRIT3 plays in the glutamate signaling cascade of the DBCs, we developed a Lrit3 ${ }^{-/-}$mouse model. An ERG will be performed on the Lrit $^{-/}$mouse to confirm a disruption in LRIT3 causes a no b-wave phenotype. Using the Lrit $^{-/}$mouse model immunohistochemistry will be performed to determine the effect deletion of LRIT3 has on signalplex proteins.

Hypothesis: LRIT3 is critical to DBC signalplex maintenance and Lrit3 ${ }^{-/}$mice will have a no b-wave ERG.

\section{Introduction}

Despite many of the molecules critical for glutamate signaling in the DBCs having been identified (Masu et al., 1995; Pardue et al., 1998; Bech-Hansen et al., 2000; Pusch et al., 2000; Gregg et al., 2007; Audo et al., 2009; Cao et al., 2009; Shen et al., 2009; van Genderen et al., 2009; Koike et al., 2010b; Okawa et al., 2010; Audo et al., 2012; Cao et al., 2012; Dhingra et al., 2012; Orlandi et al., 2012; Peachey et al., 2012b; Shen et al., 2012), there are likely additional proteins that are required to develop and maintain a functional synapse between the photoreceptors and ON BCs. At the presynaptic and 
postsynaptic membrane, proteins are arranged into a dense network consisting of scaffolding proteins, receptors, ion channels and signaling machinery. These protein networks are likely organized by scaffolding proteins that ensure the pre- and postsynaptic protein architecture are aligned to facilitate efficient signal transmission (Waites et al., 2005; McAllister, 2007; Jin and Garner, 2008). The protein network on the postsynaptic side, termed the postsynaptic density (PSD), is highly complex and consists of $\sim 1,000$ different proteins from a broad array of functional classes (Yamauchi, 2002; Yoshimura et al., 2004; Collins et al., 2005). Given that there are 10 proteins known to be critical to the mGluR6 to TRPM1 glutamate signaling cascade, additional proteins are expected to be required for structural support and organization. The absence of some of these proteins would be expected to cause cCSNB.

One such protein is LRIT3, a leucine-rich repeat, immunoglobulin like and transmembrane domain 3 protein that when absent causes cCSNB (Zeitz et al., 2013b). LRIT3 is the second leucine-rich-repeat (LRR) protein identified as being critical to the glutamate signaling cascade in the DBCs, the other being nyctalopin (Pardue et al., 1998; Bech-Hansen et al., 2000; Pusch et al., 2000; Pardue et al., 2001; Gregg et al., 2007; Cao et al., 2011; Pearring et al., 2011). Nyctalopin contains an extracellular LRR domain and is connected to the plasma membrane by a single pass transmembrane domain or a GPI anchor, depending on the species (O'Connor et al., 2005; Bojang and Gregg, 2012). Nyctalopin is required for the recruitment of TRPM1 to the DBC dendritic tips by an unknown mechanism (Gregg et al., 2007). The function of the LRR domain in nyctalopin remains to be discovered, but the importance of it can be highlighted by the fact that in humans twenty one of the thirty five cCSNB causing mutations in NYX, the gene that encodes nyctalopin, reside in regions encoding the LRR domain (Matsushima et al., 2005). 
The LRR domain is one of the most common protein domains in mammals and is highly conserved across species (Hynes and Zhao, 2000; Bjorklund et al., 2006). There are $\sim 330$ LRR-containing proteins in the human proteome, many of these being expressed in neuronal systems (Ko, 2012). The LRR domain consists of 20-29 amino acid motif that contains a conserved $L x x L x L x x N / C x L$ amino acid sequence that is typically repeated in a tandem array from 2-52 times (Kobe and Deisenhofer, 1994; Matsushima et al., 2005). The first crystal structure of a LRR protein, ribonuclease inhibitor, revealed the tandem array of repeats forms a characteristic horseshoe shaped structure (Kobe and Deisenhofer, 1993) that all known LRR proteins adopt (Matsushima et al., 2005). The concave side of this structure is formed by continuous $\beta$-sheets and the convex side often consists of $\alpha$-helices, but the composition can vary (Bella et al., 2008). LRR proteins may interact with ligands or other LRR proteins through the concave and convex surface of the horseshoe shaped structure (Matsushima et al., 2005). The LRR domain is capable of interacting with a wide range of ligands by varying the length and number of tandem repeats, which affects its curvature (Buchanan and Gay, 1996; Bella et al., 2008).

The majority of LRR proteins are expressed in the nervous system, often giving very restrictive expression patterns among neurons of the same functional type (Lauren et al., 2003; Beaubien and Cloutier, 2009; Homma et al., 2009; Hong et al., 2009). LRR proteins have been shown to play critical roles in the nervous system. TrkA, TrkB and TrkC are critical for axon guidance during development; NGL1, NGL2 and NGL3 are critical for synaptic contact formation; LGI1 is critical for stabilization of mature neurons at synapses; and NGL-3 is critical for recruitment of AMPA receptors at glutamatergic synapses (Linhoff et al., 2009; Woo et al., 2009; Siddiqui et al., 2010; de Wit et al., 2011). Mutations in LRR containing proteins lead to several neuronal diseases such as schizophrenia, epilepsy, autism, Tourette's syndrome and Alzheimer's disease (Fig. 29). 
The mechanism of how LRR proteins recruit and maintain signal complex proteins has not been determined. Further, the function and ligands of many of these LRR proteins remain unknown (de Wit et al., 2011). 
A

Axon guidance

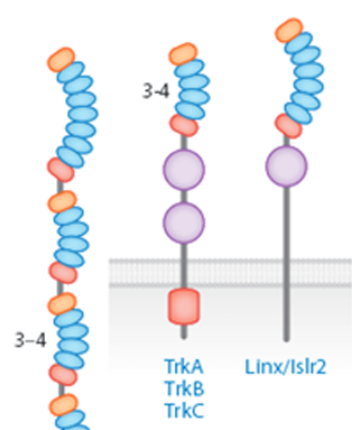

slit
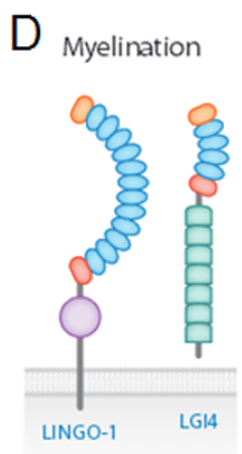

E

B
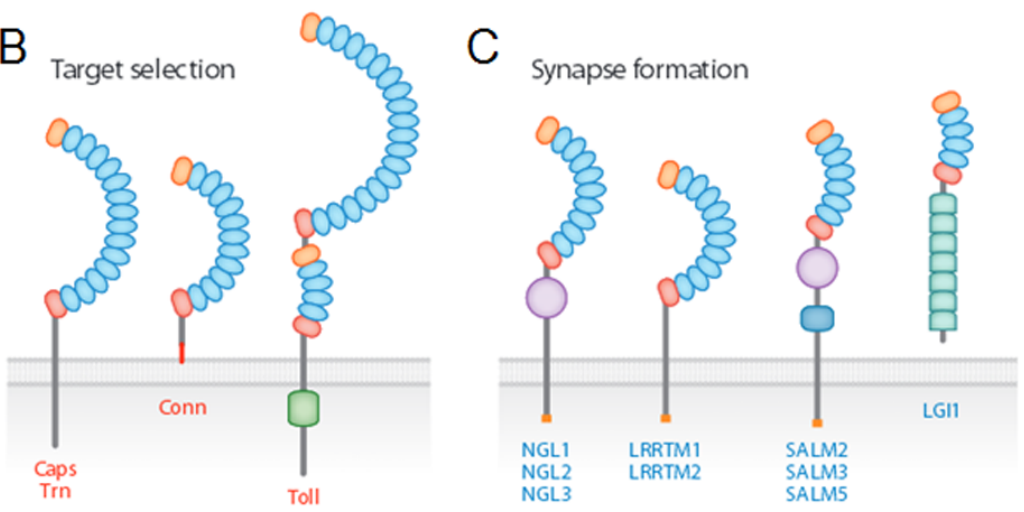

$E \underset{\substack{\text { Limiting } \\ \text { plasticity }}}{ }$

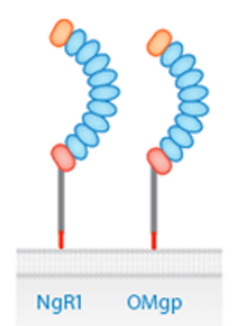

F Nervous system disorders
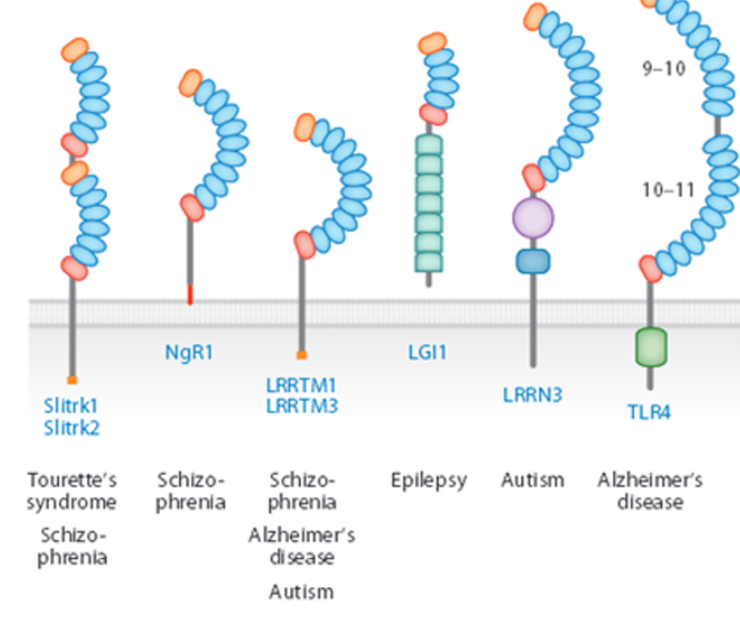

Figure 29: Overview of LRR proteins in neural circuits. LRR proteins are grouped by their known roles in neural circuits including axon guidance (A), target selection (B), synapse formation (C), myelination (D), limiting plasticity (E) and nervous system disorders (F). Different protein domains are overviewed in the diagram key (bottom left). Domain abbreviations: LRRNT and LRRCT, LRR N- and C-terminal flanking domains; Ig, immunoglobulin-like; CT3, cysteine knot; TyrK, tyrosine kinase; EPTP, epitempin; Laminin G, laminin globular; TIR, Toll/interleukin-1 receptor; EGF, epidermal growth factor-like; FN3, fibronectin type III; GPI-anchor, glycophosphatidylinositol; PDZ-IS, postsynaptic density protein. Diagram adapted from de Wit et al. (2011) 
Human patients with mutations in Lrit3 have cCSNB but are otherwise healthy, suggesting that Lrit3 may have a limited expression pattern. Because the human patients are healthy we do not need to make a conditional knockout mouse, which can be more costly and time consuming. To develop an Lrit3 ${ }^{-/}$mouse, Zinc Finger Nuclease (ZFN) technology will be utilized. ZFNs are synthetic DNA binding proteins that are fused with a non specific endonuclease (Chandrasegaran and Smith, 1999). The ZFN proteins consist of a chain of two finger modules with specificity for a DNA hexamer. The modules can be paired together to form specificity up to $24 \mathrm{bp}$. The DNA binding zinc finger modules are fused with the DNA cleavage domain of the restriction endonuclease Fokl. The Fokl restriction endonuclease must dimerize to cleave DNA (Bitinaite et al., 1998). To produce target specificity, two ZFNs must be designed on opposite strands with a 5-7 bp separation for DNA cleavage (Carroll, 2011). When the two ZFNs bind to the target site, the Fokl dimerizes and cleaves the DNA. The dependency of the restriction endonuclease to dimerize allows ZFNs to target highly specific regions within the genome and reduces off target effects. The double stranded DNA breaks created by the ZFNs induce a DNA repair mechanism, most commonly nonhomologous end joining (NHEJ), to repair the DNA double stranded break. The result of NHEJ repair can be the insertion or deletion of one to several DNA base pairs. By careful placement of target sites in an exon, some of the events will cause a frameshift resulting in a null allele.

To make a ZFN knockout mouse, ZFN mRNA is injected into a fertilized mouse single cell embryo. The mRNA is translated into the functional ZFN proteins by the embryo's cell machinery. The ZFNs then bind and cleave the targeted DNA causing double stranded breaks, followed by DNA repair. The injected embryos are transplanted into a foster mother for development. Once the pups are born they are screened for mutations at the ZFN target site by sequencing genomic DNA. 


\section{Results}

\section{Lrit3 $^{-/-}$mouse model is created using ZFN technology}

To begin the design process for generating a Lrit3 knockout mouse we cloned Lrit3 cDNA from the retina, to ensure the retinal isoform matches that present in the NCBI database. To clone Lrit3 from the retina, I designed In-Fusion Cloning (Clontech) primers that annealed to the predicted Lrit3 start and stop sites and used PCR to amplify the ORF from mouse retinal cDNA. I predicted the Lrit3 ORF in the retina was larger than the $\mathrm{NCBI}$ database annotated ORF based on next-generation transcriptome sequence analysis from retina (Brooks et al., 2011). The amplified PCR fragment was cloned into a pcDNA3.1 mammalian expression vector and sequenced using both internal and pcDNA3.1 vector primers. The sequencing results revealed the retinal isoform of Lrit3 is $363 \mathrm{bp}$ larger than the NCBI database version. The additional base pairs reside on the 5 ' end of exon 4 (Fig. 30A).

I used the SMART online resource (http://smart.embl.de/) (Letunic et al., 2012) to predict the domain structure of LRIT3 (Fig. 30B). The predicted protein structure was used to determine which protein domains may be most critical function and therefore be targeted by ZFNs to make the $\mathrm{Lrit}^{-/}$mouse. The NCBI database isoform of $L$ rit3 is 560 aa in length and produces a protein predicted to have six extracellular LRR domains, an immunoglobulin (Ig) like domain, a fibronectin type 3 domain followed by a transmembrane (TM) domain and a 76 aa cytoplasmic carboxy terminus (Fig. 30B). The retention of part of intron 3 in the retinal isoform changes the prediction of the lg like domain to an Ig C-2 Type domain and inserts regions of low complexity following the Ig domain (Fig. 30B). Regions of low complexity may provide flexibility to the extracellular domain allowing the LRR domain to bind ligands more easily.

Based on the predicted protein structure, the most critical functional domain, therefore the most desirable domain to disrupt would be the single TM domain. 
Designing ZFNs to target DNA that encodes for the TM domain would likely disrupt protein function if a missense or nonsense mutation was introduced. Targeting the ZFNs to the LRR domains would also likely knockout protein function if a missense mutation was introduced into the region because the LRR domain is likely critical to binding an extracellular ligand. Based on the predicted protein structure it was decided that ZFNs should most ideally be designed to target the LRR or TM domain to effectively eliminate protein function. 
A
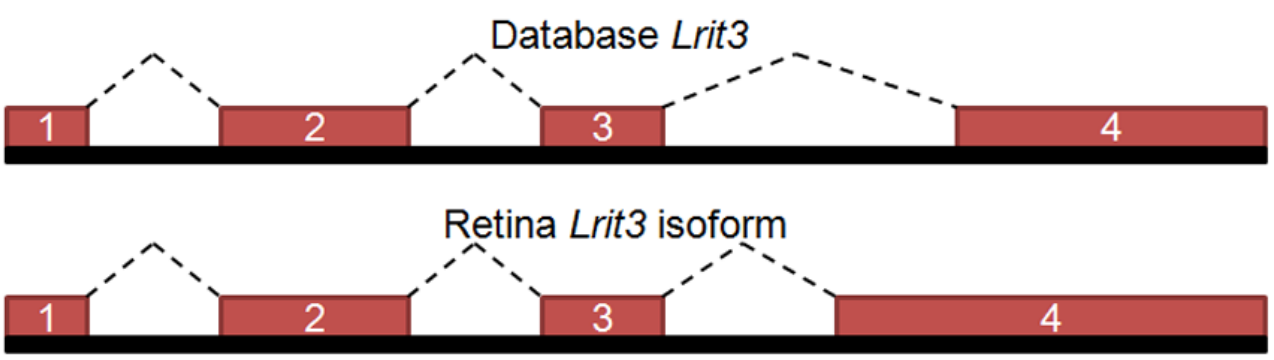

B

Database predicted LRIT3 domains

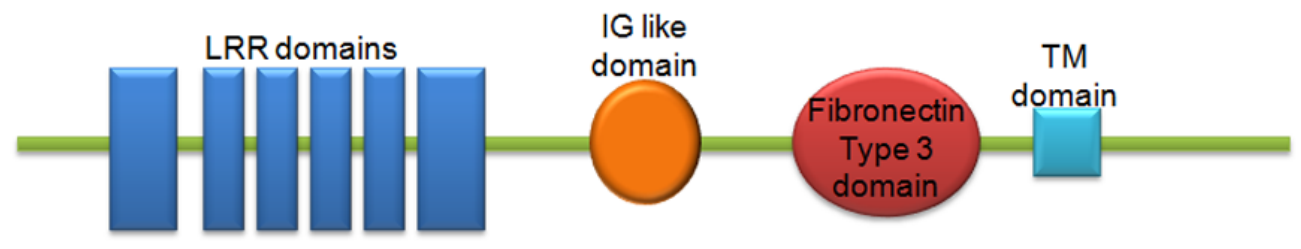

Retina predicted LRIT3 domains

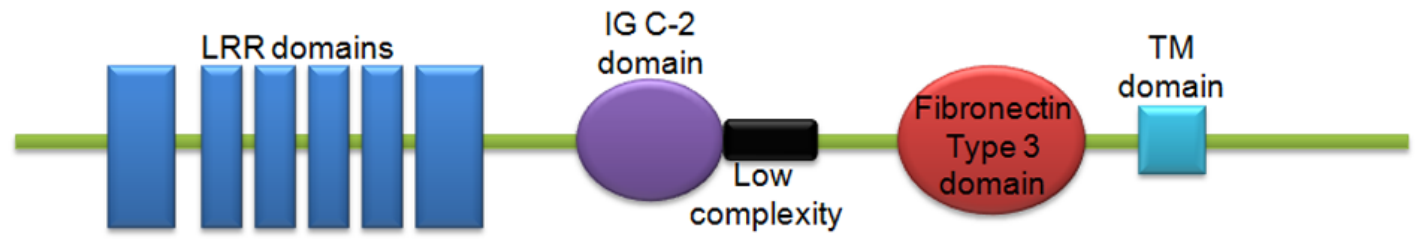

Figure 30: Predicted LRIT3 protein domains. (A) The NCBI database Lrit3 gene is $363 \mathrm{bp}$ shorter than the isoform cloned from mouse retina. The increase in size is the result of an alternative splice acceptor site in intron 3. (B) The increased size in the retinal isoform changes the IG like domain to a IG C-2 domain and inserts a region of low complexity into the protein. 
To produce a Lrit3 knockout mouse (Fig. 31), we contracted a team at SigmaAldrich to produce ZFNs. They designed and tested several ZFNs in mammalian cell lines to identify those most likely to target regions of the gene that would result in a knockout. The ZFN pair provided to us targeted the first nucleotides that encode the second LRR domain (Fig. 31). This DNA sequence encodes an amino acid sequence that is highly conserved across species (Fig 32B). At this location, missense mutations have a high probability of causing a deleterious allele by changing the secondary structure of the conserved LRR domain. The ZFN mRNAs were sent to the Fox Chase Cancer Center for creation of the knockout mouse. 
ZFN mRNA pair injected into embryo
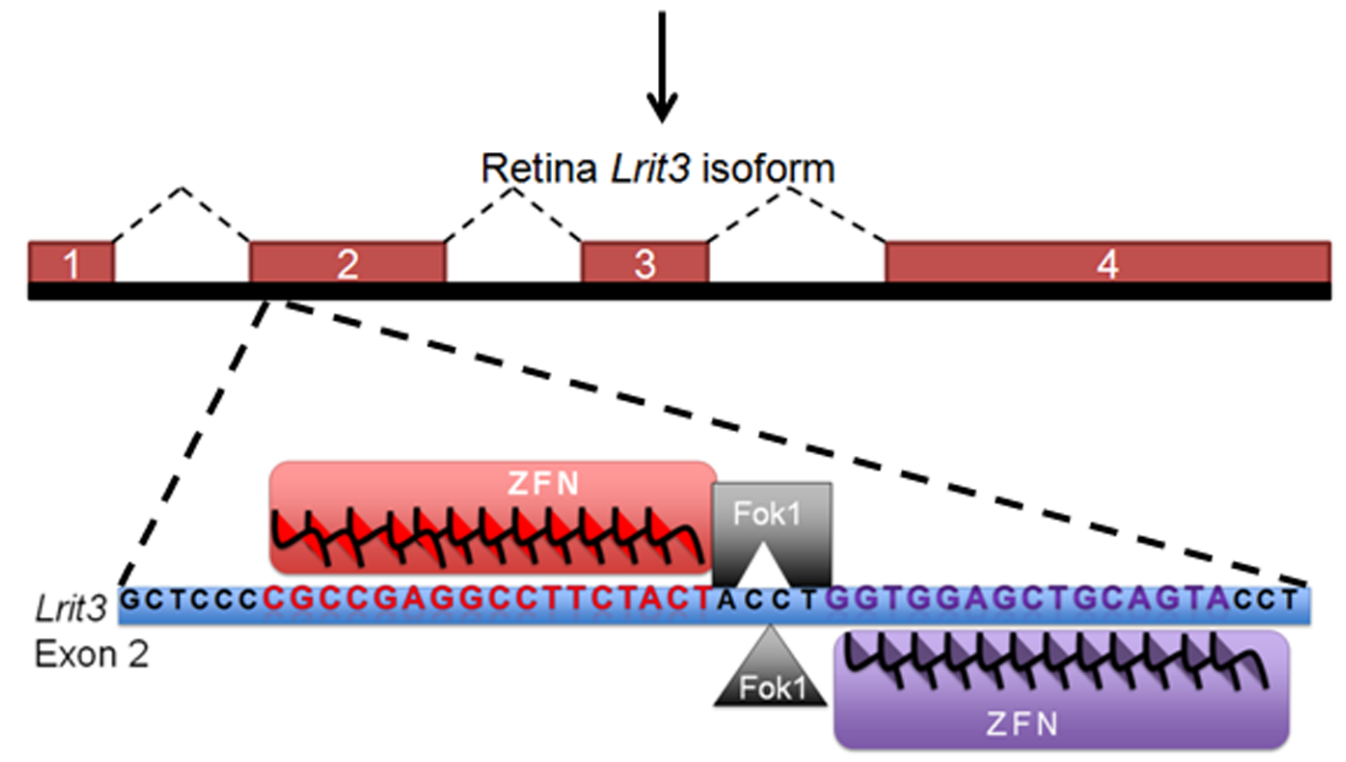

Double strand break

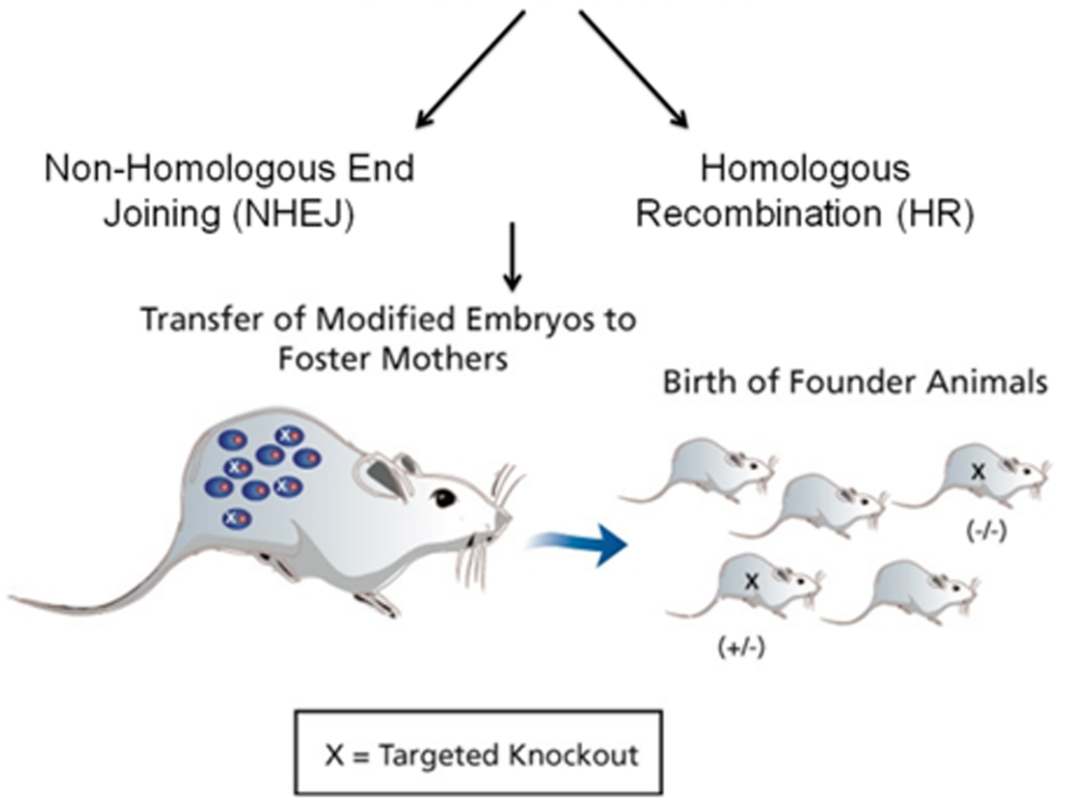

Figure 31: Workflow of the generation of a ZFN KO mouse model. ZFN mRNA pairs targeted against exon 2 of Lrit3 were injected into fertilized single cell embryos. In the embryos the mRNA is translated and the ZFNs can make double stranded breaks in the target sequence. The fertilized embryos are transferred to a foster mother for development. Modified image from Sigma-Aldrich 


\section{Genotyping ZFN induced mutations in Lrit3}

To genotype the mice, tail biopsies were obtained and genomic DNA was isolated. Primers were designed that flanked the ZFN target site and PCR products from each mouse were sequenced. Two sets of primers were designed; primer set 1 annealed $\sim 200$ bp upstream and $\sim 200$ bp downstream of the ZFN target site, primer set 2 annealed $\sim 400$ bp upstream and $\sim 400$ bp downstream of the target site (Fig. 32A). The second primer set was used to account ensure large deletions could be detected. PCR products from mice with deletions resulted in low quality sequence in regions where mutations resided. To get sequence for the individual alleles the PCR fragments were cloned into a pCR-Blunt cloning vector (Invitrogen). Several clones from each mouse were screened for mutations using Sanger Sequencing to ensure both clones of both alleles were identified.

A total of 363 embryos were injected and implanted into 9 Swiss Webster mothers. 44 offspring were obtained, 15 of which had mutations on at least one allele (Fig. 32C). The mutations ranged from a single to a $40 \mathrm{bp}$ deletion. 4 of the mice were compound heterozygotes and were predicted to be $L$ rit $3^{-/}$due to the mutations being located in a gene region that encodes a highly conserved peptide. Mouse G48 contained a $4 \mathrm{bp}$ deletion that will result in a frameshift and a $15 \mathrm{bp}$ deletion that will cause a loss of 5 highly conserved amino acids. Mouse G49 contained a complex DNA inversion that disrupts a highly conserved amino acid encoding region and a single base pair deletion that will result in a frameshift. Mouse G50 contains a 3 bp deletion that deletes a conserved tyrosine before the second LRR domain and a single bp deletion that results in a frameshift. Mouse $\mathrm{J7}$ contains a $15 \mathrm{bp}$ deletion that will cause a loss of highly conserved amino acids and a single bp deletion that will result in a frameshift.

To generate a knockout line we chose to breed mouse G47 because he carried a $40 \mathrm{bp}$ deletion on one allele, which deletes critical amino acids and produces a 
frameshift. The $40 \mathrm{bp}$ deletion also allows easy genotyping of the offspring by agarose gel electrophoresis. 
A

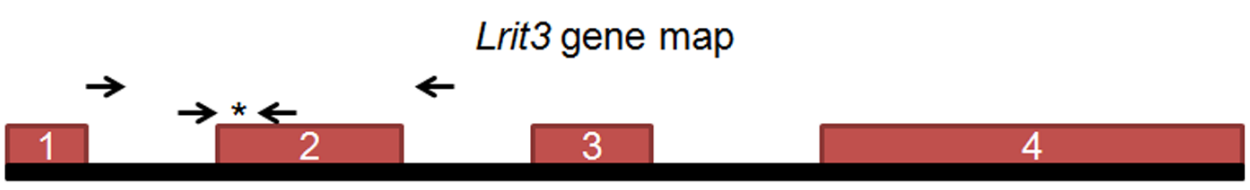

B

\begin{tabular}{|c|c|c|c|c|c|c|c|c|c|c|c|c|c|c|c|c|c|c|c|c|c|c|c|}
\hline Mus musculus & $T$ & v & v & $\mathbf{R}$ & $\mathbf{R}$ & $\mathbf{L}$ & $\mathbf{P}$ & A & E & A & $F$ & $\mathbf{Y}$ & $\mathbf{Y}$ & L & v & $\mathbf{E}$ & L & $\mathbf{Q}$ & $Y$ & L W & L & A & Y \\
\hline Homo sapiens & $T$ & $\mathbf{v}$ & 1 & $\mathbf{R}$ & $\mathbf{R}$ & 1 & $\mathbf{s}$ & A & E & A & $F$ & $\mathbf{Y}$ & $\mathbf{Y}$ & L & v & $\mathbf{E}$ & L & $\mathbf{Q}$ & Y & L W & $\mathrm{V}$ & $T$ & $\mathbf{Y}$ \\
\hline Bos taurus & $\mathrm{T}$ & v & v & $\mathbf{R}$ & $\mathbf{R}$ & 1 & $\mathbf{P}$ & A & E & A & $F$ & $\mathbf{Y}$ & $\mathbf{Y}$ & $\mathrm{L}$ & V & $\mathbf{E}$ & $\mathbf{L}$ & $\mathbf{Q}$ & Y & L W & $\mathrm{L}$ & $\mathbf{T}$ & $Y$ \\
\hline Anolis carolinensis & T & $\mathbf{v}$ & 1 & $\mathbf{R}$ & K & 1 & $\mathbf{P}$ & $\mathbf{T}$ & E & A & $F$ & $\mathbf{Y}$ & $\mathbf{Y}$ & L & v & $\mathrm{D}$ & L & $\mathbf{R}$ & $Y$ & L W & L & $\mathbf{T}$ & Y \\
\hline Rattus norvegicus & $\mathrm{T}$ & $\mathbf{v}$ & v & $\mathbf{R}$ & $\mathbf{R}$ & 1 & $\mathbf{P}$ & $\mathbf{A}$ & E & A & F & $\mathrm{Y}$ & $\mathbf{Y}$ & L & v & $\mathbf{E}$ & L & $\mathbf{Q}$ & $\mathrm{Y}$ & L W & $\mathbf{L}$ & $\mathbf{T}$ & $Y$ \\
\hline Danio rerio & $\mathrm{T}$ & $\mathbf{s}$ & V & $\mathbf{s}$ & $\mathbf{R}$ & 1 & $\mathbf{F}$ & $\mathbf{R}$ & G & A & G & $\mathbf{s}$ & A & M & $\mathbf{P}$ & E & L & L & $Y$ & L W & $L$ & $T$ & \\
\hline Gallus gallus & $\mathbf{T}$ & $\mathbf{v}$ & I & $\mathbf{R}$ & $\mathbf{R}$ & I & $\mathbf{P}$ & $\mathbf{T}$ & $E$ & A & $F$ & $\mathbf{Y}$ & $\mathbf{Y}$ & L & $v$ & D & L & $K$ & $\mathrm{Y}$ & L W & v & $T$ & $Y$ \\
\hline Xenopus tropicalis & $T$ & $\mathbf{v}$ & I & $\mathbf{R}$ & $\mathbf{K}$ & 1 & $\mathbf{P}$ & $\mathbf{T}$ & E & A & $F$ & $\mathrm{Y}$ & $Y$ & L & $v$ & D & L & $\mathrm{K}$ & $Y$ & L W & v & $T$ & \\
\hline Macaca mulatta & $\mathrm{T}$ & v & I & $\mathbf{R}$ & $\mathbf{R}$ & I & $\mathbf{L}$ & A & E & A & F & $\mathbf{Y}$ & $\mathbf{Y}$ & L & v & E & L & $\mathbf{Q}$ & Y I & L W & $\mathrm{v}$ & $\mathbf{T}$ & \\
\hline
\end{tabular}

C

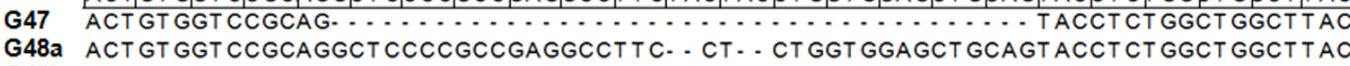
T V V R R L P A E A F Y Y L V E L Q Y W L A Y

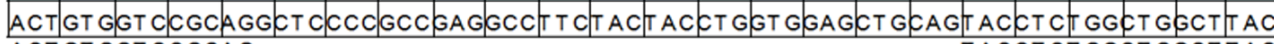
G48b ACTGT GGT CCGCAGGCT CCCCGCCGAGG............ T GGAGCT GCAGTACCT CT GGCT GGCT TAC G49a ACT GT GGT CCGCAGGCT CCCCGCCGAGGCCT TCT ACTAC- TGGT GGAGCTGCAGTACCTCT GGCT GGCT TAC G50a ACT GT GGT CCGCAGGCT CCCCGCCGAGGCCT TCTAC. - T T GGT GGAGCT GCAGTACCT CT GGCT GGCT TAC G50b ACT GT GGTCCGCAGGCT CCCCGCCGAGGCCT TCT ACACC- TGGT GGAGCT GCAGTACCTCT GGCT GGCT TAC G52 ACT GT GGT CCGCAGGCT CCCCGCCGAGGCCT TCT AC - - CT GGT GGAGCT GCAGTACCTCT GGCT GGCT TAC J1 ACT GT GGT CCGCAGGCT CCCCGCCGAGGCCT TCT ACT - - CT GGT GGAGCT GCAGTACCT CT GGCT GGCT TAC $J 4$ ACT GT GGT CCGCAGGCT CCCCGCCGAGGCCT TCT .... . T T GGT GGAGCT GCAGTACCT CT GGCT GGCT TAC J6 ACTGTGGTCCGCAGGCT CCCCGCCGAGGCCT TCTAC. - ATGGT GGAGCT GCAGTACCTCT GGCT GGCT TAC J7a ACTGTGGT CCGCAGGCT CCCCGCCGAGG............. TGGAGCT GCAGTACCTCT GGCT GGCT TAC J7b ACTGT GGT CCGCAGGCT CCCCGCCGAGGCCT TCT ACACC- TGGT GGAGCT GCAGTACCTCT GGCT GGCT TAC J12 ACT GT GGT CCGCAGGCT CCCCGCCGAGGCCT TCT ....... GGT GGAGCT GCAGTACCT CT GGCT GGCT TAC J16 ACTGTGGTCCGCAGGCT CCCCGCCGAGGCCT TCTACTAC- CGGT GGAGCT GCAGTACCTCT GGCT GGCT TAC J20 ACTGTGGT CCGCAGGCT CCCCGCCGAGGCCT................. GCAGTACCTCT GGCT GGCT TAC J24 ACTGTGGTCCGCAGGCT CCCT GCCGAGGCCT TCT ACT AC- TGGT GGAGCT GCAGTACCTCT GGCT GGCT TAC

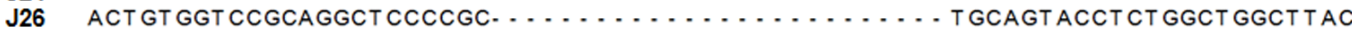
$\mathrm{J} 33$

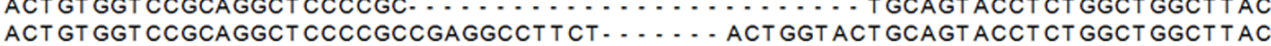

Figure 32: ZFN mutations disrupt a highly conserved protein-coding region. (A) gene map of Lrit3 including primer annealing sites used to capture ZFN targeted region (*). (B) LRIT3 amino acid alignment using the Profile Alignment (PRALINE) application (Simossis and Heringa, 2005). The ZFNs flank and cut the DNA sequence ACCT which partially encodes the Tyr/Lys amino acids (black line) that reside in a peptide sequence that is highly conserved across species. (C) Sequences from Lrit3 ZFN mutant offspring. Mutations range from single bp deletions to $\mathbf{4 0} \mathrm{bp}$ deletions. Sequence was aligned using the Clustal $\mathrm{V}$ sequence alignment (Higgins, 1994). Mouse G49b had a large inversion on one allele and is not shown. 


\section{ERG analysis reveals ZFN Lrit3 compound heterozygotes have a no b-wave ERG}

From the sequence alignment I predicted the two founder mice G48 and G49 who had a mutation on both alleles would be null for the LRIT3 protein. These were tested for visual function using the ERG. We performed a dark adapted ERG with a -0.4 $\log c d^{*} s^{*} m^{-2}$ flash stimulus. This stimulus intensity elicits a response from both rod and cone ON BCs (Stockman and Sharpe, 2006). Both mutants lacked b-waves in the ERGs, whereas littermate controls had normal ERGs (Fig. 33). These data demonstrate that similar to human (Zeitz et al., 2013b), LRIT3 is required for normal function of the DBCs in mice and that the rrit $^{-/-}$mouse is a new model of cCSNB. 


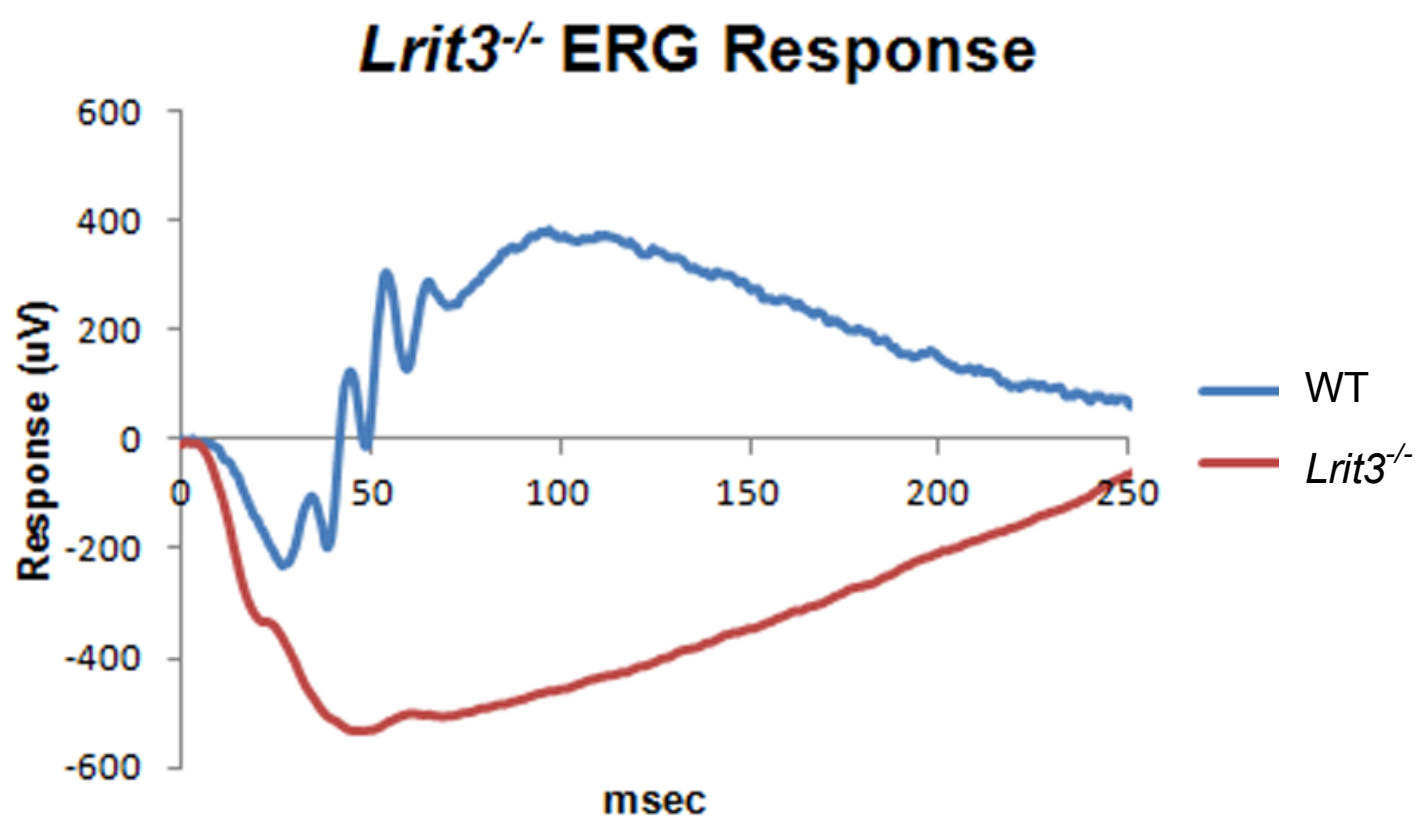

Figure 33: Predicted $L$ rit $3^{-/}$mice have a no b-wave ERG phenotype. ERG from G51 littermate WT (blue) and G48 Lrit3 $^{-/-}$(red) mice. Waveforms reflect response from dark adapted mice subjected to a $200 \mathrm{msec}-0.4 \mathrm{log} \mathrm{cd}^{*} \mathrm{~s}^{*} \mathrm{~m}^{-2}$ strobe flash. WT mice have a normal ERG a- and b-wave. The Lrit $^{-/-}$mice lack the ERG $b$-wave, indicating a disruption in DBC function. 


\section{TRPM1 does not localize to the DBC dendritic tips in the rrit3 $^{-/-}$retina}

Data from human patients with cCSNB indicated LRIT3 was critical to DBC signaling and that it was expressed at the DBC dendritic tips (Zeitz et al., 2013b). However, the mechanism by which the absence of LRIT3 disrupts glutamate signaling in the DBCs remains unknown. To determine if LRIT3 plays a critical role in localizing other proteins to the $\mathrm{DBC}$ dendritic tips, immunostaining for DBC components was performed on WT and $L$ rit $3^{--}$retinas.

Due to the similarities between LRIT3 and nyctalopin, we first investigated the expression of TRPM1 in the rrit $^{-/-}$retina. Fixed transverse retinal sections from $\mathrm{Lrit}^{-/-}$ and WT mice were labeled with antibodies against TRPM1 and mGluR6 and the proteins were visualized by confocal microscopy. As expected, TRPM1 immunostaining labeled the DBC bodies and gave punctate labeling in the OPL of WT mice. In the Lrit3 ${ }^{-/}$retina, TRPM1 was not localized to the DBC dendritic tips, although the bipolar cell bodies were positive for TRPM1 protein expression (Fig. 34). These results are similar to the TRPM1 immunostaining pattern in the $N y x^{\text {nob }}$ mice (Fig. 19, 34 and Pearring et al. (2011). 

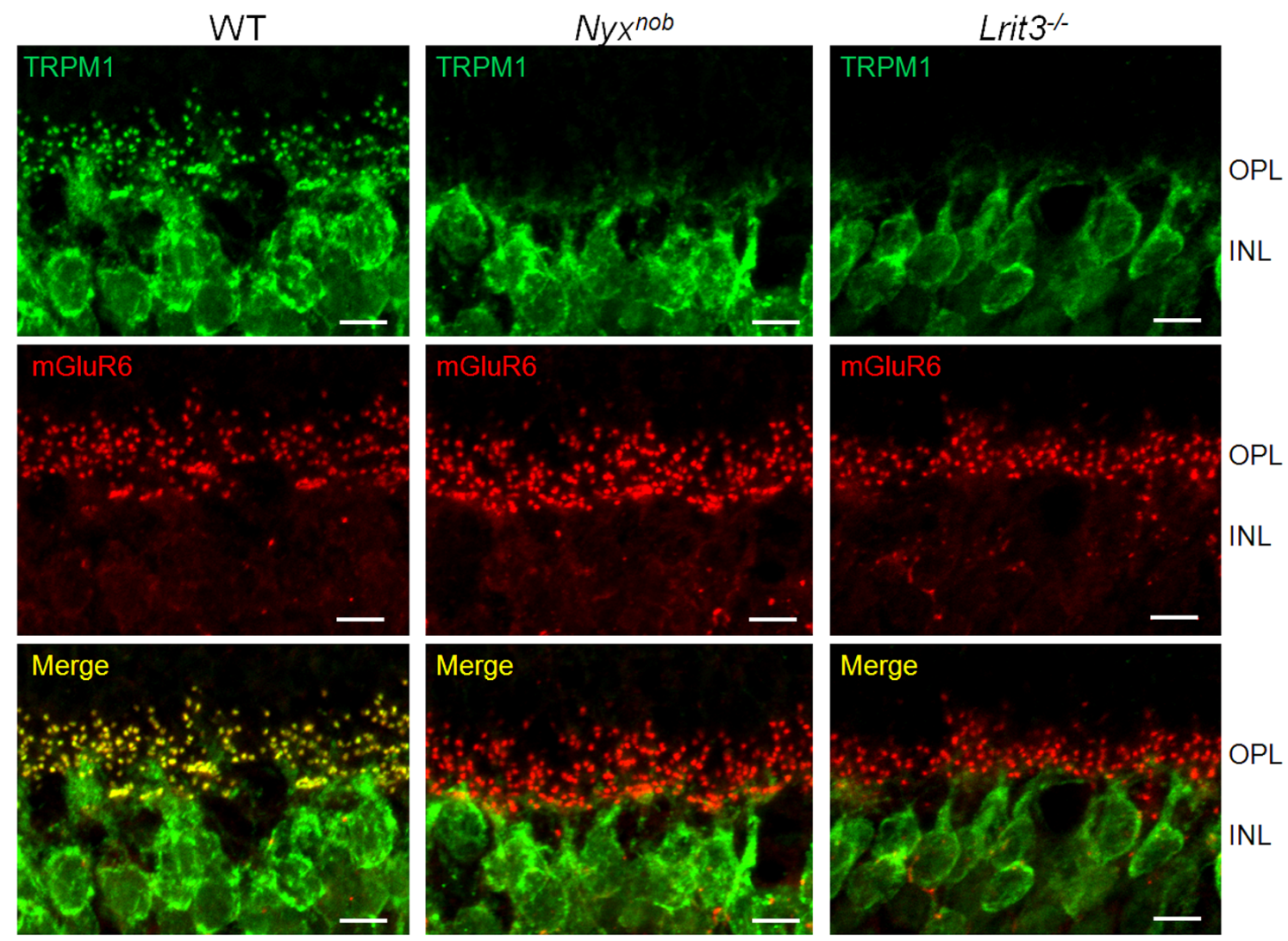

Figure 34: TRPM1 is not localized correctly in Lrit3/- DBCs. Fixed transverse retinal sections were labeled with antibodies against TRPM1 (green) and mGluR6 (red) and visualized with confocal microscopy. As expected, TRPM1 is expressed in the cell bodies and at the dendritic tips of the DBCs in WT retina and it colocalizes with mGluR6. In the $\mathrm{Lrit}^{-/-}$and $\mathrm{Nyx}^{\text {nob }}$ retinas, TRPM1 is expressed in the cell bodies but does not localize to the DBC dendritic tips and does not colocalize with mGluR6 in the OPL. Scale bar represents $5 \mu \mathrm{m}$. 


\section{Other known signalplex proteins are present in the $\mathrm{Lrit}^{-/-}$retina}

To determine if the absence of LRIT3 impacted additional signalplex proteins I labeled Lrit3 $^{-/-}$and WT transverse retinal sections with antibodies against GPR179, mGluR6, Gß5, RGS7 and RGS11 and visualized the staining with confocal microscopy. These studies showed that GPR179, mGluR6, Gß5, RGS7 and RGS11 all localized properly in the absence of LRIT3 (Fig. 35). These results mirror the expression pattern of these proteins in the $N y x^{\text {nob }}$ mouse model (Fig. 21 and (Cao et al., 2011; Pearring et al., 2011)). These data suggest that the defect in glutamate signaling in the Lrit3 $^{-/-}$ mouse is caused by the inability of TRPM1 to be localized at the DBC dendritic tips. 


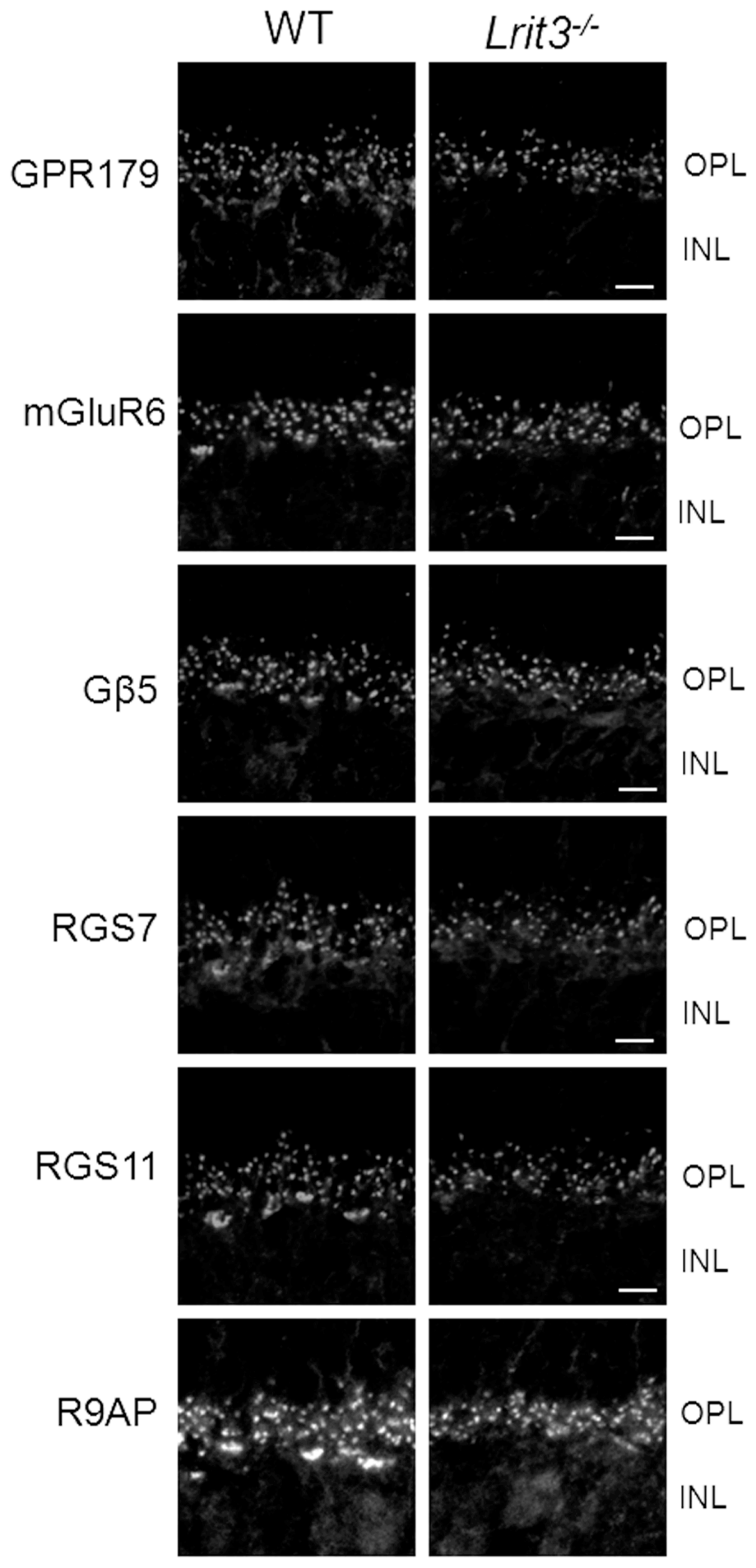

Figure 35: GPR179, mGluR6, Gß5, RGS7, RGS11 and R9AP localization is normal in Lrit $^{-/ 2}$. DBC signalplex proteins were labeled with antibodies in WT and Lrit $^{-/-}$ retinal slices and visualized using confocal microscopy. The proteins GPR179, mGluR6, Gß5, RGS7, RGS11 and R9AP are localized correctly in the Lrit3 ${ }^{-/-}$retina. 


\section{Discussion}

My experiments show that LRIT3 is required for normal DBC function in mice, consistent with the report that mutations in human Lrit3 cause cCSNB (Zeitz et al., 2013b). LRIT3 is an extracellular LRR protein with an Ig C-2 domain, fibronectin type 3 domain, and a single TM domain (Fig 30B). The molecular function of LRIT3 in the DBCs is unclear, however, I show that its expression is required for localization of the TRPM1 cation channel to the DBCs dendritic tips (Fig. 34). Like other LRR proteins (de Wit et al., 2009; Linhoff et al., 2009; Siddiqui et al., 2010), LRIT3 appears to be critical for the recruitment of synaptic components and synaptic maintenance.

Based on the immunohistochemistry data showing that TRPM1 is not localized in the Lrit3 $^{-/}$DBCs, we anticipated the rod BCs would not respond to the mGluR6 antagonist, CPPG, or the TRPM1 modulator, capsaicin. To test whether the rod BCs respond to CPPG in the Lrit3 $^{-/}$retina, transverse retinal sections were prepared for single cell recordings. Retinal slices were bathed in a solution containing L-AP4, which maximally activates the glutamate signaling cascade and closes the TRPM1 channel. Application of $0.6 \mathrm{mM} \mathrm{CPPG}$ for $200 \mathrm{msec}$ elicited a response in the $\mathrm{Lrit}^{-/-}$retina that was significantly decreased compared to WT. This response was not significantly different than the response from $\operatorname{Trpm} 1^{-/-}$rod BCs but was significantly smaller than the response generated in the Gpr179 ${ }^{\text {nob5 }}$ rod BCs (Fig. 36A,B). These results support our data that the TRPM1 channel is not localized to the DBC dendritic tips in the Gpr179 nob5 retinas.

To further test whether TRPM1 is not localized to the DBC dendritic tips in the rod $\mathrm{BCs}$ of the $\mathrm{Lrit}^{-/}$retina we measured the rod $\mathrm{BC}$ response to exogenous capsaicin application. Capsaicin is a modulator of the TRPM1 channel. If the channel is localized to the plasma membrane of the rod $\mathrm{BC}$ dendritic tips in the Lrit $3^{-/}$retina it should have a capsaicin response greater than the $T r p m 1^{-/-}$capsaicin response. We found that the 
capsaicin response in rod $\mathrm{BCs}$ from $\mathrm{Lrit}^{-/-}$retina were no different than the capsaicin response in the $\operatorname{Trpm} 1^{-/-}$rod BCs, and as expected both responses are significantly decreased compared to WT (Fig. 36C,D). The capsaicin response in the Lrit3 ${ }^{-/}$rod BCs was not significantly different from the response in Gpr179 nob5 $\operatorname{rod}$ BCs (Fig. 36D). These data further support that in the Lrit $^{-/}$retina the absence of TRPM1 channel localization is the cause of the cell's inability to respond to light. 
A

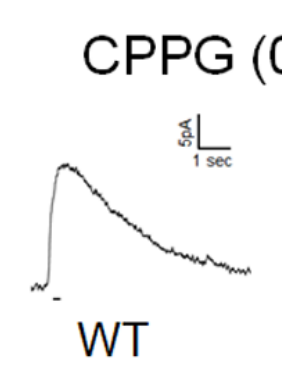

B

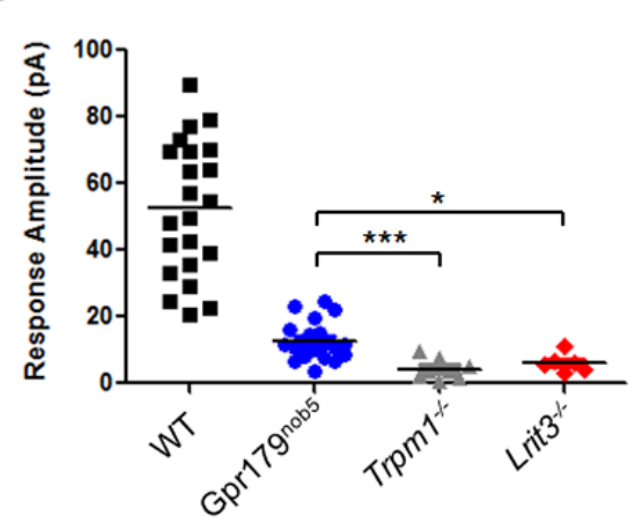

C

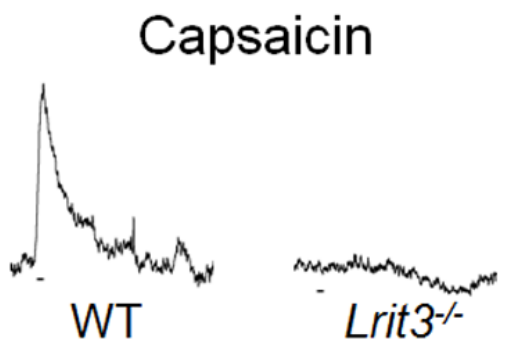

D

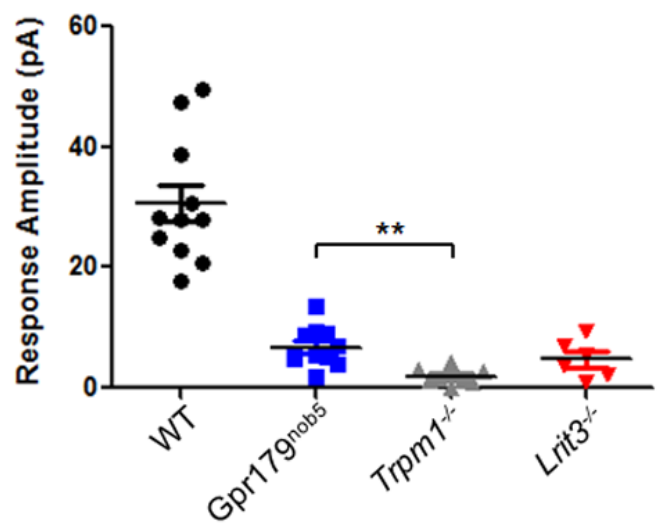

Figure 36: rrit $^{-/}$rod BC response to CPPG and capsaicin. Whole cell recordings from WT, Gpr179 $9^{\text {nob5 }}$, Trpm 1 ${ }^{-/}$and Lrit3 $3^{-/}$rod BCs in the presence of L-AP4. (A) Representative traces of WT and Lrit3 ${ }^{-/}$rod BCs in response to CPPG. (B) Scatter diagrams of peak amplitude responses. One way ANOVA $\left.{ }^{* * *}\right)$ : $\operatorname{Trpm} 1^{-1 /}$ is significantly decreased in amplitude compared to Gpr179 ${ }^{\text {nob5 }}$. ( $\left.{ }^{*}\right)$ Lrit3 $^{-/-}$is significantly decreased in amplitude compared to Gpr179 ${ }^{\text {nob5 }}$. LRIT3 does not significantly differ from Trpm $1^{-1 /}$. (C) Representative traces of WT and Lrit $3^{-/}$rod BCs in response to capsaicin. (D) Scatter diagrams of peak amplitude responses. One way ANOVA $\left({ }^{* *}\right)$ : Gpr179 ${ }^{n o b 5}$ and Trpm $1^{\%-}$ are significantly different. Lrit3 $3^{-/}$ does not significantly differ from either $G p r 179^{\text {nob5 }}$ or Trpm1 ${ }^{1 / 2}$. Experiments performed by Kathryn Heath. 
Like LRIT3, nyctalopin is critical for the localization of TRPM1 at the DBC dendritic tips. It will be important to determine the mechanism by which LRIT3 is responsible for the localization of TRPM1, whether the function of LRIT3 is redundant with nyctalopin, and if LRIT3 serves a purpose beyond localization of the TRPM1 channel. Determining the mechanism by which LRIT3 localizes the TRPM1 channel to the DBC dendritic tips will be important because the general mechanism by which LRR proteins recruit other proteins to the synapse is largely unknown.

Leucine-rich repeat transmembrane protein 1 (LRRTM1) is responsible for the assembly of excitatory synapses. LRRTM1 plays a role in recruiting the AMPA receptor to the plasma membrane and is responsible for long-term potentiation in pyramidal neurons (Soler-Llavina et al., 2013). In the BCs, we know that the number of TRPM1 channels at the DBC dendritic tips rate limits the response (Peachey et al., 2012a). It will be important to determine if LRIT3 plays a role in regulating the number of TRPM1 channels at the DBC dendritic tips, possibly shaping DBC plasticity.

LRR proteins are known to have roles in synapse maintenance (de Wit et al., 2011), therefore, It will be important to perform electron microscopy (EM) on the rrit3 $^{-/-}$ retinas to ensure they are formed properly and maintained through adulthood. The Lrit3 / line should also be crossed with the $N y x^{\text {nob }}$ mouse and EM performed. Deleting both LRIT3 and nyctalopin from the DBC dendritic tips may cause the synapse to be less stable, especially if both proteins have non-redundant roles in synapse maintenance. These experiments would help us understand if LRIT3 has a function beyond recruiting the TRPM1 channel to the DBC dendritic tips.

To study LRIT3 function further an antibody must be made or a transgenic mouse expressing a tagged LRIT3 protein produced to do immunohistochemistry and biochemistry. With the emergence of new genome editing techniques this can be efficiently accomplished and provide a reliable method of detecting LRIT3 in vivo. The 
tag would ideally be placed on the carboxy terminus. The carboxy terminus is a short intracellular domain likely only needed for anchoring the protein to the membrane and addition of a protein tag should not interfere with protein function.

There are several protein domains in LRIT3 in which a functional role needs to be identified, including the LRR domain, Ig C-2 domain and Fibronectin type 3 domain (Fig. 30). The extracellular LRR domain is likely critical for ligand binding in the EC matrix. The Ig C-2 domain of LRIT3 likely is a protein interaction domain. Ig domains of synaptic proteins have been demonstrated to be critical for stabilization of synaptic protein complexes (Rapti et al., 2011). Fibronectin type 3 domains have been shown to play critical roles in neural synaptic plasticity (Strekalova et al., 2002; Dityatev et al., 2010), calcium channel potentiation and integrin binding to the EC matrix (Dityatev et al., 2010). Genome editing techniques can be used to create dominant negative LRIT3 transgenic mice that contain mutations in the different protein domains. Ideally, these mutations would disrupt a protein domain but allow the protein to localize properly. These experiments would help differentiate which domains are critical for TRPM1 and LRIT3 localization.

\section{Methods}

\section{DNA isolation}

For mouse genotyping, tail samples were collected from mice and placed in a 0.7 $\mathrm{mL}$ microcentrifuge tube. To the tube, $200 \mu \mathrm{L}$ of DirectPCR (Viagen Biotech) was added along with $2 \mu \mathrm{L}$ proteinase $\mathrm{K}(20 \mathrm{mg} / \mathrm{ml})$. Sample was incubated for at least $4 \mathrm{~h}$ at $55^{\circ} \mathrm{C}$ in a dry bath with brief vortexing every $h$. After the tail sample was adequately digested, the sample was heated to $85^{\circ} \mathrm{C}$ for $30 \mathrm{~min}$ to inactivate the proteinase $\mathrm{K}$. Samples were then ready for PCR.

\section{General PCR}


PCR was performed using either Phire II or Phusion taq polymerase (Thermo Scientific). Reactions were made to $20 \mu \mathrm{l}$ consisting of $0.5 \mu \mathrm{M}$ of each forward and reverse primers, $200 \mu \mathrm{M}$ of dNTPs, $4 \mu \mathrm{l}$ of $5 \mathrm{X}$ reaction buffer, and either $0.4 \mu \mathrm{l}$ of Phire II or Phusion DNA Polymerase, template DNA and water varied by application. Reactions were run on a Bio Rad C1000 Touch Thermal Cycler.

\section{RNA Isolation from Retina}

Mice were euthanized by $\mathrm{CO}_{2}$ inhalation and cervical dislocation. Eyes were extracted and enucleated and the retinas were removed and stored at $-80^{\circ} \mathrm{C}$ in RNAlater (Ambion). RNA was isolated according to the manufacturers protocol for the PureLink® RNA Mini Kit (Ambion). Briefly, retinal tissue was removed from RNAlater and homogenized in 10-20 volumes of Tri Reagent (Ambion) by pipetting. The homogenized mixture was incubated for $5 \mathrm{~min}$ at room temperature then $200 \mu \mathrm{l}$ chloroform per $\mathrm{ml}$ of homogenate was added and the mixture vortexed, and incubated for an additional 5 min at room temperature; followed by centrifugation at $12,000 \times \mathrm{g}$ for $10 \mathrm{~min}$ at $4^{\circ} \mathrm{C}$. The aqueous phase volume was transferred to a new $1.5 \mathrm{ml}$ centrifuge tube and $200 \mu \mathrm{l}$ of $100 \%$ ethanol was added to the sample and vortexed for $15 \mathrm{sec}$. The sample was placed in a filter cartridge (Ambion) and centrifuged at $12,000 \times \mathrm{g}$ for $30 \mathrm{sec}$. Flow through was discarded and $500 \mu \mathrm{l}$ wash solution was applied to the filter cartridge and centrifuged at $12,000 \times \mathrm{g}$ for $30 \mathrm{sec}$. The wash step was repeated and filter cartridge was transferred to a new microcentrifuge tube and $100 \mu$ of Elution buffer was applied to elute the RNA. The RNA was collected by centrifugation for $30 \mathrm{sec}$ at $12,000 \mathrm{x} \mathrm{g}$, and stored at $-80^{\circ} \mathrm{C}$.

\section{Zero Blunt pCR Cloning}

pCR-Blunt cloning was performed according the manufacturers instructions (Invitrogen MAN0007763). In brief, ligation reactions were set up containing $1 \mu \mathrm{l}$ pCRBlunt vector, $1 \mu \mathrm{l}$ of blunt-end PCR product, $2 \mu \mathrm{l}$ XX ExpressLink T4 DNA Ligase Buffer, 
$5 \mu$ sterile water and $1 \mu$ l ExpressLink T4 DNA Ligase. The ligation reaction was gently mixed and incubated at room temperature for 10 minutes. In the meantime, One Shot Top10 cells (Invitrogen) were thawed on ice. $2.5 \mu \mathrm{l}$ of the ligation reaction was added to one vial of One Shot Top10 cells and mixed by swirling. Cells were incubated on ice for $15 \mathrm{~min}$ then heat shocked for $30 \mathrm{sec}$ at $42^{\circ} \mathrm{C}$ and returned to ice for at least 1 minute. $250 \mu \mathrm{l}$ of SOC medium was added to the transformed cells and they were incubated at $37^{\circ} \mathrm{C}$ while shaking at $225 \mathrm{rpm}$ for $1 \mathrm{~h}$. Cells were then plated on Circlegrow agarose plates (MP Biomedicals) supplemented with kanamycin $100 \mu \mathrm{g} / \mathrm{mL}$.

\section{In-Fusion HD Cloning}

PCR fragments were amplified using primers designed to have 14-18 bp 5' overhangs that share homology with the cut site on a linearized vector. PCR fragments were treated with Cloning Enhancer (Clontech) and incubated at $37^{\circ} \mathrm{C}$ for 15 min then at $80^{\circ} \mathrm{C}$ for $15 \mathrm{~min}$ on a PCR thermal cycler. Linearized vector, treated PCR fragment and water was added to $5 \mathrm{X}$ In-Fusion HD Enzyme Premix according to manufacturers suggestion (Clontech). The reaction was mixed by pipetting and incubated for $15 \mathrm{~min}$ at $50^{\circ} \mathrm{C}$ on a thermal cycler. The reaction mix was placed on ice or stored at $-20^{\circ} \mathrm{C}$ until transformation.

\section{Plasmid Isolation}

Plasmid purification was performed using the Wizard Plus SV Minipreps DNA Purification System (Promega) according to the manufacturers protocol. Single colonies from transformed bacteria were selected from agarose plates using a sterile toothpicks and used to inoculate $2 \mathrm{ml}$ of Circlegrow media supplemented with $100 \mu \mathrm{g} / \mathrm{ml}$ kanamycin. The culture was grown overnight at $37^{\circ} \mathrm{C}$ while shaking at $225 \mathrm{RPM}$. The bacterial cells were transferred to a $2 \mathrm{ml}$ centrifuge tube and pelleted by centrifugation at max speed for 5 minutes to pellet cells. Supernatant was discarded and cells were resupsended by vortexing in $250 \mu$ of Cell Resuspension Solution. $250 \mu \mathrm{l}$ of Cell Lysis 
Solution and $10 \mu \mathrm{l}$ Alkaline Protease Solution was added, and mixed by inverting tubes several times then incubated at room temperature for 5 min. $350 \mu \mathrm{l}$ of Neutralization Solution then was added to the tube and mixed by inverting several times. Tubes were centrifuged at $12,000 \times \mathrm{g}$ for $10 \mathrm{~min}$ to pellet cellular debris and the supernatant was decanted into a spin column. The spin column was placed into a collection tube and centrifuged for $1 \mathrm{~min}$ at $12,000 \mathrm{xg}$. The flow through was discarded and the plasmid bound to the column was washed 2 times by adding $500 \mu \mathrm{l}$ of Column Wash Solution to the column and centrifugation for $1 \mathrm{~min}$ at $12,000 \mathrm{xg}$. The spin column was transferred to a clean microcentrifuge tube and $100 \mu \mathrm{l}$ of sterile water was added to the column to elute plasmid DNA. The water was incubated on the column for $1 \mathrm{~min}$ at room temperature then centrifuged for $1 \mathrm{~min}$ at $12,000 \mathrm{xg}$ to collect DNA. DNA was stored at $-20^{\circ} \mathrm{C}$.

\section{Antibodies}

The following primary antibodies and their concentrations were used: sheep antiGPR179 (peptide KVQEETPGEDLDRPVLQKR), 1:2,000 (Peachey et al., 2012b); guinea pig anti-mGluR6 1:1,000 (Koike et al., 2010b); sheep anti-TRPM1 1:1,000 (Cao et al., 2011); goat anti-Gß5 (peptide MATDGLHENETLASLKC), 1:1,000 (Morgans et al., 2007); goat anti-R9AP 1:1,000 (Hu and Wensel, 2002); sheep anti-RGS11 (peptide CSPALQSTPREPAATSSPEGADGE), 1:1,000 (Martemyanov et al., 2005); sheep antiRGS7 1:1,000 (Morgans et al., 2007).

Secondary antibodies (Invitrogen, 1:1,000) appropriate to each primary antibody included: donkey anti-sheep Alexa-488, donkey anti-rabbit Alexa-680, donkey anti-rabbit Alexa-546, donkey anti-mouse Alexa-647 and donkey anti-guinea pig Cy3 (Millipore, $1: 1,000)$

\section{Immunohistochemistry}


Mice were euthanized with $\mathrm{CO}_{2}$ followed by cervical dislocation. Their eyes were enucleated and the lens was removed. Eyecups were washed in phosphate buffered saline (PBS) then fixed for 30 min in a $4 \%$ formaldehyde PBS solution ( $\mathrm{pH} \mathrm{7.4).} \mathrm{Eyecups}$ were washed 3 times with PBS then cryoprotected in increasing concentrations of sucrose in PBS (10\%, 15\% for $1 \mathrm{~h}$ each and 30\% overnight). Eyecups were embedded in 2:1 OCT/ $20 \%$ sucrose PBS solution frozen in a liquid nitrogen cooled bath of isopentane. Eyecups were sectioned $(18 \mu \mathrm{m})$ using a Leica 1850 cryostat. Sections were mounted on glass slides (SuperFrost) and allowed to dry for 10 mintues at room temperature before being stored at $-80^{\circ} \mathrm{C}$. Sections were air dried at $37^{\circ} \mathrm{C}$ for $15 \mathrm{~min}$ and washed with PBS and PBS containing $0.05 \%$ Triton X-100 (PBX) for 5 min each, then blocked in PBX plus 5\% normal donkey serum blocking solution for $1 \mathrm{~h}$. Sections were incubated overnight at room temperature in the presence of the primary antibody diluted in blocking solution, then washed 3 times for 10 min each with PBX followed by incubation in secondary antibody in blocking solution for $1 \mathrm{~h}$ at room temperature. Sections were washed $2 \times 10$ min in PBX and 1 X PBS. Slides were cover-slipped using Immu-Mount (Thermo Scientific) and slides were imaged using an Olympus FV1000 confocal microscope. Images were universally adjusted for brightness using Photoshop. 


\section{CHAPTER V: SUMMARY}

I showed in Aim I that a mutation in Gpr179 was the cause of the no b-wave phenotype in the Gpr179 $9^{\text {nob5 }}$ mouse. The Gpr179 ${ }^{\text {nob5 }}$ mouse contains a $\sim 6.5 \mathrm{~kb}$ insertion in intron 1 of the gene Gpr179 that is thought to disrupt mRNA processing between exon 1/2. Analysis of gene expression using qRT-PCR revealed exon $1 / 2$ expression was greatly decreased in the Gpr179 ${ }^{\text {nob5 }}$ mouse. Morpholino disruption of GPR179 protein translation in zebrafish resulted in a greatly diminished ERG b-wave compared to scrambled control. Human patients with cCSNB were screened for mutations in Gpr179 and two patients were identified with inactivating mutations on each allele. These data indicate that Gpr179 ${ }^{\text {nob5 }}$ is a new model of cCSNB.

I determined Gpr179 is expressed in the INL of the retina using in situ hybridization. Immunohistochemistry followed by confocal microscopy revealed the protein is localized to the tips of the DBCs in the OPL and GPR179 puncta juxtapose RIBEYE staining and colocalize with mGluR6. Finally, we generated a Gpr179-/ mouse model to confirm its role in DBC function. The line is currently being generated at the University of Louisville.

In Aim II I examined the interdependency of DBC signalplex components on the expression of other cascade components using immunohistochemistry and confocal microscopy. I found that the localization of RGS7, RGS11, Gß5 and R9AP to the DBC dendritic tips was dependent on expression of GPR179 (Fig. 21). Through collaboration we showed that GPR179 interacts with RGS7/Gß5, RGS11/Gß5 (Appendix II and Orlandi et al., (2012)), further supporting our hypothesis that GPR179 acts to 
recruit and localize the RGS proteins to the DBC signalplex. I showed that GPR179 forms complexes with mGluR6 and TRPM1 using the Duolink PLA Assay. Similar conclusions were made by Orlandi et al. (2013). In Ray et al. (2013) (Appendix III) we showed that GPR179 and TRPM1 IP together from retinal lysates. Taken together, it appears GPR179 acts as a hub and recruits the RGS proteins to the DBC signalplex and brings them within close proximity of mGluR6 and TRPM1. Localizing all of the signalplex components into a macromolecular complex enhances the efficiency and fidelity of signaling between the mGluR6 receptor and the TRPM1 channel.

The finding that R9AP does not localize in the $R G S 7^{-/} / R G S 11^{-/} \mathrm{DBC}$ is an interesting finding and suggests that the RGS complex consisting of R9AP, G 35 and RGS7 or 11 must form in an intracellular compartment before the complex can localize at the DBC dendritic tips. The RGS complex cannot form and does not localize properly if Gß5 (Chen et al., 2003) or RGS7/11 are absent (Fig. 22). It was previously reported that R9AP localization in the DBCs was decreased in a hypomorphic $R G S 7^{-/ /} / R G S 11^{-/-}$ mouse (Zhang et al., 2010) but It was later determined that the mouse still retained RGS7 function.

The small ERG b-wave response in the Gpr179 nob5 mice suggested that mGluR6 was capable of signaling to TRPM1 in the absence of GPR179 (Ray et al. 2013 and Appendix III). Using whole cell patch clamp we confirmed that mGluR6 could signal to TRPM1, albeit with reduced sensitivity in both the Gpr $179^{n o b 5}$ and $R G S 7^{-/} / R G S 11^{-/}$rod BCs (Ray et al., 2013 and Appendix III). This reduced response is the result of the absence of RGS proteins in both models. The capsaicin response in the rod BCs displays another role for GPR179 in the DBCs. In the Gpr179 ${ }^{\text {nob5 }}$ rod BCs the capsaicin response is significantly decreased compared to WT, whereas in the $R G S 7^{-/ /} / R G S 11^{-/-}$ rod BCs the capsaicin response is no different than WT. These data indicate that 
TRPM1 has reduced sensitivity to capsaicin because the TRPM1 interaction with GPR179 enhances the sensitivity of the channel to gating.

In Aim III we created a new mouse model of cCSNB based on a report of a newly identified gene being involved in DBC function in humans (Zeitz et al., 2013b). The new mouse model was created using ZFNs, which allowed the quickest and most efficient generation of a knockout animal. Using ZFN technology, we created several compound Lrit3 mutants that had a no b-wave ERG. We selected a Lrit3 mutant with a 40 bp deletion to generate the knockout line.

The gene Lrit3 encodes a single pass transmembrane protein with an extracellular LRR domain, similar to another LRR containing protein critical to DBC function, nyctalopin. We found that LRIT3 may serve a similar role as nyctalopin in the DBCs because both are required for TRPM1 localization at the DBC dendritic tips. We confirmed that TRPM1 is absent from the DBC dendritic tips in the Lrit $^{-{ }^{--}}$mouse using whole cell recordings in response capsaicin puffs. These experiments indicate the disruption in the LRIT3 mutant is due to the absence of the TRPM1 channel. The mechanism by which LRIT3 controls TRPM1 localization to the DBC dendritic tips will be an important further line of investigation. Because the amount of TRPM1 at the DBC dendritic tips is known to set the light response amplitude (Peachey et al., 2012a), it will be important to determine if LRIT3 can actively change the amount of TRPM1 at the $\mathrm{DBC}$ dendritic tips in response to changes in light levels.

\section{Model of DBC signaling}

Using the knowledge gained from this project, we have developed a more thorough understanding of the rod BC signalplex protein arrangement and mechanism of signaling. When I started the project we had a very limited understanding of how the mGluR6 receptor signaled to the TRPM1 cation channel in the DBCs (Fig 5). Over the last 4 years we identified 2 new proteins, GPR179 and LRIT3, involved in DBC signaling 
(Peachey et al., 2012b) and characterized the interdependency of the DBC signalplex proteins (Orlandi et al., 2012). We used physiology in models of DBC dysfunction to understand the role of individual DBC signalplex proteins (Appendix III and Ray et al., (2013)). These data were used to assemble a more accurate model of the DBC signalplex and how it may function (Fig. 37)

We propose that GPR179 is the focal point of the signalplex protein scaffold. GPR179 interacts with TRPM1, mGluR6 and is responsible for recruiting the RGS machinery in close proximity to TRPM1 and mGluR6. The LRR proteins nyctalopin and LRIT3 play a critical role in recruiting TRPM1 to the DBC dendritic tips and they likely interact with extracellular matrix proteins to position TRPM1 at the synapse. The glutamate receptor mGluR6 is physically associated with this complex and likely interacts with one or more proteins through a transmembrane domain. The G proteins are tethered to the membrane through posttranslational modifications.

In the dark, photoreceptors are continuously releasing glutamate into the synaptic cleft where it becomes bound by mGluR6. This induces a conformational change in the receptor that allows it to bind $G \alpha_{0} G D P$ and activate it to $G \alpha_{0}$ GTP by GTP exchange. The newly formed $\mathrm{Ga}_{\circ} \mathrm{GTP}$ is released from the receptor and the heterotrimeric $\mathrm{G}$ protein dissociates into $\mathrm{G} \alpha_{0} \mathrm{GTP}$ and the $\mathrm{G} \beta \mathrm{y}$ dimer. The Gßy complex binds and gates the TRPM1 channel closed in a transient manner. The RGS proteins through their constitutive GTPase activity immediately inactivate the $\mathrm{Ga}_{0} \mathrm{GTP}$ subunit. The $G \alpha_{0} G D P$ subunit reforms the heterotrimeric complex with Gßy. For gating of the TRPM1 channel to occur, activation of $\mathrm{Ga}_{\circ}$ must outpace the constitutive RGS GTPase activity. Once the equilibrium shifts in favor of the RGS proteins through a decrease in glutamate release by the photoreceptors, the TRPM1 channel opens, causing the cell to depolarize. 
Whether the cell is depolarized or hyperpolarized depends on the equilibrium of open to closed TRPM1 channels. When the glutamate signaling cascade is maximally activated by the presence of glutamate in the synaptic cleft, the equilibrium favors closed TRPM1 channels because $G$ protein activation out paces the constitutive RGS activity. In the absence of glutamate in the synaptic cleft, $G$ protein activation ceases and the RGS proteins quickly deactivate any remaining active $G$ proteins. The equilibrium shifts to mostly inactive $G$ proteins and the TRPM1 channel opens, producing the light response. 


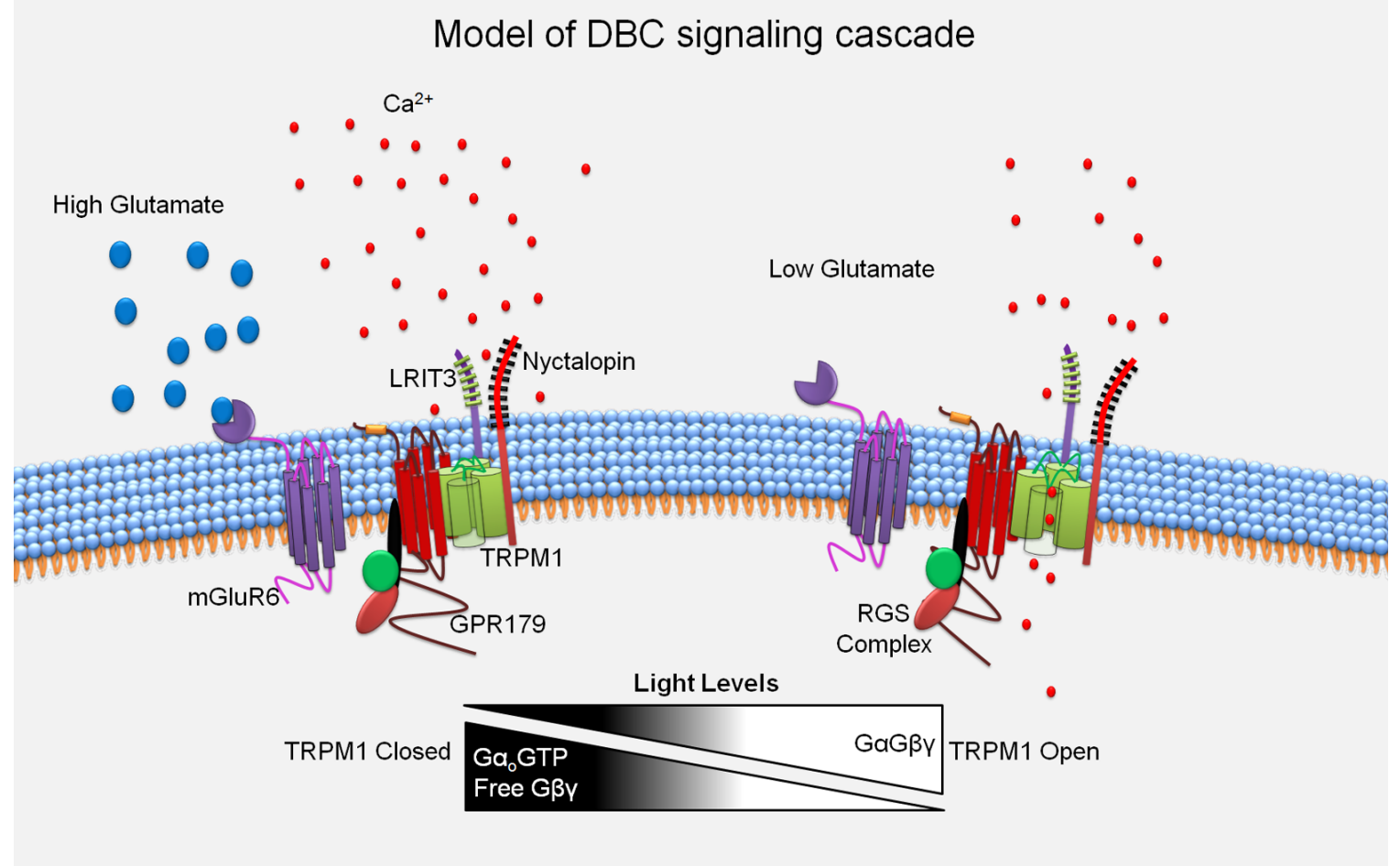

Figure 37: Model of $D B C$ signaling. The ratio of free $G \beta y$ to $G \alpha_{0} G \beta y$ determines the gating of the TRPM1 channel. In the dark glutamate in the synaptic cleft activates the mGluR6 receptor, which activates $G$ proteins. Free G $\beta y$ transiently binds and gates the TRPM1 channel closed. In order for TRPM1 channels to be gated closed and keep the cell hyperpolarized $G$ protein activation must outpace the constitutive RGS deactivation of $G$ proteins. When glutamate is decreased in the synaptic cleft, $G$ protein activation by mGluR6 is halted and the RGS proteins quickly deactivate any remaining active $G$ proteins. $G$ protein suppression of the TRPM1 channel is relieved causing the channel to open and the cell to depolarize. GPR179 serves to anchor the RGS complex in close proximity with the TRPM1 channel for increased gating sensitivity. GPR179 interaction with TRPM1 enhances the sensitivity of the channel to gating. LRIT3 and nyctalopin serve to localize the TRPM1 channel at the synapse. 


\section{Future directions}

This study has answered many questions regarding glutamate signaling in the DBCs. Just as important, it creates new questions that, upon answering, will give rise to a greater understanding of DBC function. It will be important to explore the role of the extracellular EGF domain of GPR179. Instead of GPR179 binding a ligand, this domain may be critical for interaction with the extracellular matrix or other proteins on the pre or postsynaptic side of the synapse. The large intracellular carboxy terminus of GPR179 is a large and contains an amino acid sequence of low complexity that likely gives it a disordered secondary structure. The current literature suggests that large scaffolding proteins often contain a disordered structure in solution, but upon binding their interacting partners they create a highly ordered protein scaffold (Dyson and Wright, 2005; Xie et al., 2007; Coletta et al., 2010; Ferreon et al., 2013). It will be important to identify the carboxy terminus-binding partners to gain a deeper understanding of signalplex architecture.

The $\mathrm{GABA}_{\mathrm{B} 1}$ and $\mathrm{GABA}_{\mathrm{B} 2}$ GPCRs form a heterodimer and are both required to carry out signaling by a trans-activating mechanism that involves one receptor binding the ligand and the other receptor activating the $\mathrm{G}$ proteins (Margeta-Mitrovic et al., 2000; Pagano et al., 2001; Rondard et al., 2008; Monnier et al., 2011). It will be interesting to determine if GPR179 and mGluR6 are capable of functioning in this manner. While it seems unlikely that GPR179 binds glutamate due to its lack of extracellular glutamate binding domain, it is possible that it can still play a role in the activation of G proteins because it is predicted to couple to $\mathrm{Ga}_{\mathrm{i} / \mathrm{o}} \mathrm{G}$ proteins.

The gating mechanism of Gßy on the TRPM1 channel will be important to determine. At this time it is unknown where the $G \beta y$ binding site on TRPM1 is located and what its stoichiometry is with TRPM1. Biophysical techniques such as fluorescent spectroscopy may be able to characterize this interaction. 
The extracellular LRR domains of LRIT3 and nyctalopin are likely critical to protein function, yet nothing is known about their function in the DBCs. Understanding what these proteins interact with in the extracellular matrix will provide a greater understanding about how they coordinate the localization of proteins on both sides of the synapse. Crossing the Lrit3 $^{--}$mouse with various mouse models of cCSNB and transgenic models can help pinpoint the function of LRIT3. It will be important to cross the LRIT3 mouse with the $\operatorname{Tg}(\text { Gabrr1-YFP/nyx })^{\text {Rgg1 }}$ mouse, which expresses a YFP tagged nyctalopin protein (Gregg et al., 2007), to determine if nyctalopin is localized to the DBC dendritic tips in the $L$ rit3 ${ }^{-/}$mouse. 


\section{REFERENCES}

Agte S, Junek S, Matthias S, Ulbricht E, Erdmann I, Wurm A, Schild D, Kas JA, Reichenbach A (2011) Muller glial cell-provided cellular light guidance through the vital guinea-pig retina. Biophys J 101:2611-2619.

Ahnelt P, Kolb H (1994a) Horizontal cells and cone photoreceptors in primate retina: a Golgi-light microscopic study of spectral connectivity. J Comp Neurol 343:387-405.

Ahnelt P, Kolb H (1994b) Horizontal cells and cone photoreceptors in human retina: a Golgi-electron microscopic study of spectral connectivity. J Comp Neurol 343:406-427.

Albert TJ, Molla MN, Muzny DM, Nazareth L, Wheeler D, Song X, Richmond TA, Middle CM, Rodesch MJ, Packard CJ, Weinstock GM, Gibbs RA (2007) Direct selection of human genomic loci by microarray hybridization. Nat Methods 4:903-905.

Aloisi F (2001) Immune function of microglia. Glia 36:165-179.

Alpadi K, Magupalli VG, Kappel S, Koblitz L, Schwarz K, Seigel GM, Sung CH, Schmitz F (2008) RIBEYE recruits Munc119, a mammalian ortholog of the Caenorhabditis elegans protein unc119, to synaptic ribbons of photoreceptor synapses. J Biol Chem 283:26461-26467.

Altier C (2012) GPCR and Voltage-Gated Calcium Channels (VGCC) Signaling Complexes. Sub-cellular biochemistry 63:241-262.

Altier C, Zamponi GW (2008) Signaling complexes of voltage-gated calcium channels and G protein-coupled receptors. J Recept Signal Transduct Res 28:71-81.

Anant JS, Ong OC, Xie HY, Clarke S, O'Brien PJ, Fung BK (1992) In vivo differential prenylation of retinal cyclic GMP phosphodiesterase catalytic subunits. J Biol Chem 267:687-690.

Anderson GR, Posokhova E, Martemyanov KA (2009) The R7 RGS protein family: multi-subunit regulators of neuronal $G$ protein signaling. Cell Biochem Biophys 54:33-46.

Applebury ML, Antoch MP, Baxter LC, Chun LL, Falk JD, Farhangfar F, Kage K, Krzystolik MG, Lyass LA, Robbins JT (2000) The murine cone photoreceptor: a single cone type expresses both $\mathrm{S}$ and $\mathrm{M}$ opsins with retinal spatial patterning. Neuron 27:513-523.

Arshavsky VY, Burns ME (2012) Photoreceptor signaling: supporting vision across a wide range of light intensities. J Biol Chem 287:1620-1626.

Attwell D, Tessier-Lavigne M, Wilson M, Mobbs P (1987) Bipolar cell membrane currents and signal processing in the axolotl retina. Neurosci Res Suppl 6:S191-204. 
Audet M, Bouvier M (2012) Restructuring G-protein- coupled receptor activation. Cell 151:14-23.

Audo I, Robson AG, Holder GE, Moore AT (2008) The negative ERG: clinical phenotypes and disease mechanisms of inner retinal dysfunction. Survey of ophthalmology 53:16-40.

Audo I et al. (2009) TRPM1 is mutated in patients with autosomal-recessive complete congenital stationary night blindness. Am J Hum Genet 85:720-729.

Audo I et al. (2012) Whole-exome sequencing identifies mutations in GPR179 leading to autosomal-recessive complete congenital stationary night blindness. Am J Hum Genet 90:321-330.

Awatramani GB, Slaughter MM (2000) Origin of transient and sustained responses in ganglion cells of the retina. J Neurosci 20:7087-7095.

Ayoub GS, Copenhagen DR (1991) Application of a fluorometric method to measure glutamate release from single retinal photoreceptors. J Neurosci Methods 37:7-14.

Baehr W, Wu SM, Bird AC, Palczewski K (2003) The retinoid cycle and retina disease. Vision Res 43:2957-2958.

Bahadori R, Biehlmaier O, Zeitz C, Labhart T, Makhankov YV, Forster U, Gesemann M, Berger W, Neuhauss SC (2006) Nyctalopin is essential for synaptic transmission in the cone dominated zebrafish retina. Eur J Neurosci 24:16641674.

Ball SL, Gregg RG (2002) Using mutant mice to study the role of voltage-gated calcium channels in the retina. Adv Exp Med Biol 514:439-450.

Ball SL, Powers PA, Shin HS, Morgans CW, Peachey NS, Gregg RG (2002) Role of the beta(2) subunit of voltage-dependent calcium channels in the retinal outer plexiform layer. Invest Ophthalmol Vis Sci 43:1595-1603.

Barlow HB, Hill RM (1963) Selective sensitivity to direction of movement in ganglion cells of the rabbit retina. Science 139:412-414.

Barlow HB, Hill RM, Levick WR (1964) Retinal Ganglion Cells Responding Selectively to Direction and Speed of Image Motion in the Rabbit. J Physiol 173:377-407.

Barnes S, Kelly ME (2002) Calcium channels at the photoreceptor synapse. Adv Exp Med Biol 514:465-476.

Bayburt TH, Leitz AJ, Xie G, Oprian DD, Sligar SG (2007) Transducin activation by nanoscale lipid bilayers containing one and two rhodopsins. J Biol Chem 282:14875-14881.

Bayley PR, Morgans CW (2007) Rod bipolar cells and horizontal cells form displaced synaptic contacts with rods in the outer nuclear layer of the nob2 retina. J Comp Neurol 500:286-298.

Beatty S, Nolan J, Kavanagh H, O'Donovan $O$ (2004) Macular pigment optical density and its relationship with serum and dietary levels of lutein and zeaxanthin. Archives of biochemistry and biophysics 430:70-76.

Beatty S, Boulton M, Henson D, Koh HH, Murray IJ (1999) Macular pigment and age related macular degeneration. Br J Ophthalmol 83:867-877.

Beaubien F, Cloutier JF (2009) Differential expression of Slitrk family members in the mouse nervous system. Developmental dynamics : an official publication of the American Association of Anatomists 238:3285-3296. 
Bech-Hansen NT, Naylor MJ, Maybaum TA, Pearce WG, Koop B, Fishman GA, Mets M, Musarella MA, Boycott KM (1998) Loss-of-function mutations in a calciumchannel alpha1-subunit gene in Xp11.23 cause incomplete X-linked congenital stationary night blindness. Nat Genet 19:264-267.

Bech-Hansen NT, Naylor MJ, Maybaum TA, Sparkes RL, Koop B, Birch DG, Bergen AA, Prinsen CF, Polomeno RC, Gal A, Drack AV, Musarella MA, Jacobson SG, Young RS, Weleber RG (2000) Mutations in NYX, encoding the leucine-rich proteoglycan nyctalopin, cause X-linked complete congenital stationary night blindness. Nat Genet 26:319-323.

Bella J, Hindle KL, McEwan PA, Lovell SC (2008) The leucine-rich repeat structure. Cell Mol Life Sci 65:2307-2333.

Berman DM, Gilman AG (1998) Mammalian RGS proteins: barbarians at the gate. J Biol Chem 273:1269-1272.

Berson DM (2003) Strange vision: ganglion cells as circadian photoreceptors. Trends Neurosci 26:314-320.

Berson DM (2008) 1.25 - Retinal Ganglion Cell Types and Their Central Projections. In: The Senses: A Comprehensive Reference (Volume Editors: Allan IB, Akimichi K, Gordon MS, Gerald W, Thomas DA, Richard HM, Peter D, Donata O, Stuart F, Gary KB, Bushnell MC, Jon HK, Esther GardnerA2 - Volume Editors: Allan I. Basbaum AKGMSGWTDARHMPDDOSFG, eds), pp 491-519. New York: Academic Press.

Berson DM, Dunn FA, Takao M (2002) Phototransduction by retinal ganglion cells that set the circadian clock. Science 295:1070-1073.

Besch D, Jagle H, Scholl HP, Seeliger MW, Zrenner E (2003) Inherited multifocal RPEdiseases: mechanisms for local dysfunction in global retinoid cycle gene defects. Vision Res 43:3095-3108.

Bijveld MM, van Genderen MM, Hoeben FP, Katzin AA, van Nispen RM, Riemslag FC, Kappers AM (2013a) Assessment of night vision problems in patients with congenital stationary night blindness. PLoS One 8:e62927.

Bijveld MM, Florijn RJ, Bergen AA, van den Born LI, Kamermans M, Prick L, Riemslag FC, van Schooneveld MJ, Kappers AM, van Genderen MM (2013b) Genotype and Phenotype of 101 Dutch Patients with Congenital Stationary Night Blindness. Ophthalmology.

Bill BR, Petzold AM, Clark KJ, Schimmenti LA, Ekker SC (2009) A Primer for Morpholino Use in Zebrafish. Zebrafish 6:69-77.

Bitinaite J, Wah DA, Aggarwal AK, Schildkraut I (1998) FokI dimerization is required for DNA cleavage. Proc Natl Acad Sci U S A 95:10570-10575.

Bjorklund AK, Ekman D, Elofsson A (2006) Expansion of protein domain repeats. Plos Comput Biol 2:e114.

Blanks JC, Hageman GS, Johnson LV, Spee C (1988) Ultrastructural visualization of primate cone photoreceptor matrix sheaths. J Comp Neurol 270:288-300.

Bojang P, Jr., Gregg RG (2012) Topological analysis of small leucine-rich repeat proteoglycan nyctalopin. PLoS One 7:e33137.

Bok D (1993) The retinal pigment epithelium: a versatile partner in vision. Journal of cell science Supplement 17:189-195. 
Boulton M, Dayhaw-Barker P (2001) The role of the retinal pigment epithelium: topographical variation and ageing changes. Eye (Lond) 15:384-389.

Bouvier M (2001) Oligomerization of G-protein-coupled transmitter receptors. Nat Rev Neurosci 2:274-286.

Boycott BB, Dowling JE, Kolb H (1969) Organization of the Primate Retina: Light Microscopy. Philosophical transactions of the Royal Society of London Series B, Biological sciences 255:109-184.

Briggman KL, Helmstaedter M, Denk W (2011) Wiring specificity in the directionselectivity circuit of the retina. Nature 471:183-188.

Bringmann A, Pannicke T, Grosche J, Francke M, Wiedemann P, Skatchkov SN, Osborne NN, Reichenbach A (2006) Muller cells in the healthy and diseased retina. Prog Retin Eye Res 25:397-424.

Bringmann A, Pannicke T, Biedermann B, Francke M, Iandiev I, Grosche J, Wiedemann P, Albrecht J, Reichenbach A (2009) Role of retinal glial cells in neurotransmitter uptake and metabolism. Neurochem Int 54:143-160.

Brooks MJ, Rajasimha HK, Roger JE, Swaroop A (2011) Next-generation sequencing facilitates quantitative analysis of wild-type and $\mathrm{Nrl}(-/-)$ retinal transcriptomes. Mol Vis 17:3034-3054.

Brown KT (1969) The electroretinogram: its components and their origins. UCLA forum in medical sciences 8:319-378.

Brown KT, Murakami M (1964) A New Receptor Potential of the Monkey Retina with No Detectable Latency. Nature 201:626-628.

Brown PK, Wald G (1963) Visual Pigments in Human and Monkey Retinas. Nature 200:37-43.

Buchanan SG, Gay NJ (1996) Structural and functional diversity in the leucine-rich repeat family of proteins. Progress in biophysics and molecular biology 65:144.

Burchett SA (2000) Regulators of G protein signaling: a bestiary of modular protein binding domains. J Neurochem 75:1335-1351.

Burkhardt DA (1994) Light adaptation and photopigment bleaching in cone photoreceptors in situ in the retina of the turtle. J Neurosci 14:1091-1105.

Burkhardt DA, Gottesman J (1987) Light adaptation and responses to contrast flashes in cones of the walleye retina. Vision Research 27:1409-1420.

Bush RA, Sieving PA (1994) A proximal retinal component in the primate photopic ERG a-wave. Invest Ophthalmol Vis Sci 35:635-645.

Cabrera JL, de Freitas F, Satpaev DK, Slepak VZ (1998) Identification of the Gbeta5RGS7 protein complex in the retina. Biochem Biophys Res Commun 249:898902.

Cajal SR (1892a) The Structure of the Retina.

Cajal SR (1892b) The structure of the retina: C. C. Thomas.

Cao Y, Posokhova E, Martemyanov KA (2011) TRPM1 Forms Complexes with Nyctalopin In Vivo and Accumulates in Postsynaptic Compartment of ONBipolar Neurons in mGluR6-Dependent Manner. J Neurosci 31:11521-11526.

Cao Y, Song H, Okawa H, Sampath AP, Sokolov M, Martemyanov KA (2008) Targeting of RGS7/Gbeta5 to the dendritic tips of ON-bipolar cells is independent of its association with membrane anchor R7BP. J Neurosci 28:10443-10449. 
Cao Y, Pahlberg J, Sarria I, Kamasawa N, Sampath AP, Martemyanov KA (2012) Regulators of G protein signaling RGS7 and RGS11 determine the onset of the light response in ON bipolar neurons. Proc Natl Acad Sci U S A 109:79057910.

Cao Y, Masuho I, Okawa H, Xie K, Asami J, Kammermeier PJ, Maddox DM, Furukawa T, Inoue T, Sampath AP, Martemyanov KA (2009) Retina-specific GTPase accelerator RGS11/G beta 5S/R9AP is a constitutive heterotrimer selectively targeted to mGluR6 in ON-bipolar neurons. J Neurosci 29:9301-9313.

Carroll D (2011) Genome engineering with zinc-finger nucleases. Genetics 188:773782.

Carter-Dawson LD, LaVail MM (1979) Rods and cones in the mouse retina. I. Structural analysis using light and electron microscopy. J Comp Neurol 188:245-262.

Chabre M, le Maire M (2005) Monomeric G-protein-coupled receptor as a functional unit. Biochemistry 44:9395-9403.

Chandrasegaran S, Smith J (1999) Chimeric restriction enzymes: what is next? Biological chemistry 380:841-848.

Chang B, Heckenlively JR, Bayley PR, Brecha NC, Davisson MT, Hawes NL, Hirano AA, Hurd RE, Ikeda A, Johnson BA, McCall MA, Morgans CW, Nusinowitz S, Peachey NS, Rice DS, Vessey KA, Gregg RG (2006) The nob2 mouse, a null mutation in Cacna1f: anatomical and functional abnormalities in the outer retina and their consequences on ganglion cell visual responses. Vis Neurosci 23:11-24.

Chavez AE, Diamond JS (2008) Diverse mechanisms underlie glycinergic feedback transmission onto rod bipolar cells in rat retina. J Neurosci 28:7919-7928.

Chen CK (2005) The vertebrate phototransduction cascade: amplification and termination mechanisms. Reviews of physiology, biochemistry and pharmacology 154:101-121.

Chen CK, Woodruff ML, Chen FS, Chen D, Fain GL (2010) Background light produces a recoverin-dependent modulation of activated-rhodopsin lifetime in mouse rods. J Neurosci 30:1213-1220.

Chen CK, Burns ME, Spencer M, Niemi GA, Chen J, Hurley JB, Baylor DA, Simon MI (1999) Abnormal photoresponses and light-induced apoptosis in rods lacking rhodopsin kinase. Proc Natl Acad Sci U S A 96:3718-3722.

Chen CK, Eversole-Cire P, Zhang H, Mancino V, Chen YJ, He W, Wensel TG, Simon MI (2003) Instability of GGL domain-containing RGS proteins in mice lacking the G protein beta-subunit Gbeta5. Proc Natl Acad Sci U S A 100:6604-6609.

Choe HW, Kim YJ, Park JH, Morizumi T, Pai EF, Krauss N, Hofmann KP, Scheerer P, Ernst OP (2011) Crystal structure of metarhodopsin II. Nature 471:651-655.

Chun L, Zhang WH, Liu JF (2012) Structure and ligand recognition of class C GPCRs. Acta pharmacologica Sinica 33:312-323.

Chung KY, Rasmussen SG, Liu T, Li S, DeVree BT, Chae PS, Calinski D, Kobilka BK, Woods VL, Jr., Sunahara RK (2011) Conformational changes in the G protein Gs induced by the beta2 adrenergic receptor. Nature 477:611-615.

Clarke RJ, Ikeda H (1985) Luminance and darkness detectors in the olivary and posterior pretectal nuclei and their relationship to the pupillary light reflex 
in the rat. I. Studies with steady luminance levels. Experimental brain research Experimentelle Hirnforschung Experimentation cerebrale 57:224232.

Coletta A, Pinney JW, Solis DY, Marsh J, Pettifer SR, Attwood TK (2010) Lowcomplexity regions within protein sequences have position-dependent roles. BMC systems biology 4:43.

Collins MO, Yu L, Coba MP, Husi H, Campuzano I, Blackstock WP, Choudhary JS, Grant SG (2005) Proteomic analysis of in vivo phosphorylated synaptic proteins. J Biol Chem 280:5972-5982.

Conti M, Beavo J (2007) Biochemistry and physiology of cyclic nucleotide phosphodiesterases: essential components in cyclic nucleotide signaling. Annu Rev Biochem 76:481-511.

Copenhagen DR, Jahr CE (1989) Release of endogenous excitatory amino acids from turtle photoreceptors. Nature 341:536-539.

Cowan CW, Fariss RN, Sokal I, Palczewski K, Wensel TG (1998) High expression levels in cones of RGS9, the predominant GTPase accelerating protein of rods. Proc Natl Acad Sci U S A 95:5351-5356.

Curcio CA, Hendrickson AE (1991) Chapter 5 Organization and development of the primate photoreceptor mosaic. Progress in Retinal Research 10:89-120.

Curcio CA, Sloan KR, Kalina RE, Hendrickson AE (1990) Human photoreceptor topography. J Comp Neurol 292:497-523.

Curcio CA, Allen KA, Sloan KR, Lerea CL, Hurley JB, Klock IB, Milam AH (1991) Distribution and morphology of human cone photoreceptors stained with anti-blue opsin. J Comp Neurol 312:610-624.

Dacey DM (2004) Origins of perception: retinal ganglion cell diversity and the creation of parallel visual pathways: Bradford Book.

Dacheux RF, Raviola E (1986) The rod pathway in the rabbit retina: a depolarizing bipolar and amacrine cell. J Neurosci 6:331-345.

Dalke C, Loster J, Fuchs H, Gailus-Durner V, Soewarto D, Favor J, Neuhauser-Klaus A, Pretsch W, Gekeler F, Shinoda K, Zrenner E, Meitinger T, Hrabe de Angelis M, Graw J (2004) Electroretinography as a screening method for mutations causing retinal dysfunction in mice. Invest Ophthalmol Vis Sci 45:601-609.

Dartnall HJ, Lythgoe JN (1965) The spectral clustering of visual pigments. Vision Res 5:81-100.

de Wit J, Hong W, Luo L, Ghosh A (2011) Role of leucine-rich repeat proteins in the development and function of neural circuits. Annual review of cell and developmental biology 27:697-729.

de Wit J, Sylwestrak E, O'Sullivan ML, Otto S, Tiglio K, Savas JN, Yates JR, 3rd, Comoletti D, Taylor P, Ghosh A (2009) LRRTM2 interacts with Neurexin1 and regulates excitatory synapse formation. Neuron 64:799-806.

DeVries SH (2000) Bipolar cells use kainate and AMPA receptors to filter visual information into separate channels. Neuron 28:847-856.

DeVries SH, Schwartz EA (1999) Kainate receptors mediate synaptic transmission between cones and 'Off' bipolar cells in a mammalian retina. Nature 397:157160. 
Dhingra A, Lyubarsky A, Jiang M, Pugh EN, Jr., Birnbaumer L, Sterling P, Vardi N (2000) The light response of ON bipolar neurons requires G[alpha]o. J Neurosci 20:9053-9058.

Dhingra A, Ramakrishnan H, Neinstein A, Fina ME, Xu Y, Li J, Chung DC, Lyubarsky A, Vardi N (2012) Gbeta3 is required for normal light ON responses and synaptic maintenance. J Neurosci 32:11343-11355.

Dick 0, tom Dieck S, Altrock WD, Ammermuller J, Weiler R, Garner CC, Gundelfinger ED, Brandstatter JH (2003) The presynaptic active zone protein bassoon is essential for photoreceptor ribbon synapse formation in the retina. Neuron 37:775-786.

Dityatev A, Schachner M, Sonderegger P (2010) The dual role of the extracellular matrix in synaptic plasticity and homeostasis. Nat Rev Neurosci 11:735-746.

Doan T, Azevedo AW, Hurley JB, Rieke F (2009) Arrestin competition influences the kinetics and variability of the single-photon responses of mammalian rod photoreceptors. J Neurosci 29:11867-11879.

Dornonville de la Cour M (1993) Ion transport in the retinal pigment epithelium. A study with double barrelled ion-selective microelectrodes. Acta Ophthalmol Suppl:1-32.

Doumazane E, Scholler P, Zwier JM, Eric T, Rondard P, Pin JP (2011) A new approach to analyze cell surface protein complexes reveals specific heterodimeric metabotropic glutamate receptors. FASEB J 25:66-77.

Dryja TP, McGee TL, Berson EL, Fishman GA, Sandberg MA, Alexander KR, Derlacki DJ, Rajagopalan AS (2005) Night blindness and abnormal cone electroretinogram ON responses in patients with mutations in the GRM6 gene encoding mGluR6. Proc Natl Acad Sci U S A 102:4884-4889.

Dyson HJ, Wright PE (2005) Intrinsically unstructured proteins and their functions. Nature reviews Molecular cell biology 6:197-208.

Ecker JL, Dumitrescu ON, Wong KY, Alam NM, Chen SK, LeGates T, Renna JM, Prusky GT, Berson DM, Hattar S (2010) Melanopsin-expressing retinal ganglion-cell photoreceptors: cellular diversity and role in pattern vision. Neuron 67:4960.

Ehinger B, Ottersen OP, Storm-Mathisen J, Dowling JE (1988) Bipolar cells in the turtle retina are strongly immunoreactive for glutamate. Proc Natl Acad Sci U S A 85:8321-8325.

Elstrott J, Anishchenko A, Greschner M, Sher A, Litke AM, Chichilnisky EJ, Feller MB (2008) Direction selectivity in the retina is established independent of visual experience and cholinergic retinal waves. Neuron 58:499-506.

Euler T, Detwiler PB, Denk W (2002) Directionally selective calcium signals in dendrites of starburst amacrine cells. Nature 418:845-852.

Famiglietti EV, Jr., Kolb H (1975) A bistratified amacrine cell and synaptic cirucitry in the inner plexiform layer of the retina. Brain Res 84:293-300.

Famiglietti EV, Jr., Kaneko A, Tachibana M (1977) Neuronal architecture of on and off pathways to ganglion cells in carp retina. Science 198:1267-1269.

Farber DB, Lolley RN (1974) Cyclic guanosine monophosphate: elevation in degenerating photoreceptor cells of the C3H mouse retina. Science 186:449451. 
Ferreon AC, Ferreon JC, Wright PE, Deniz AA (2013) Modulation of allostery by protein intrinsic disorder. Nature 498:390-394.

Fesenko EE, Kolesnikov SS, Lyubarsky AL (1985) Induction by cyclic GMP of cationic conductance in plasma membrane of retinal rod outer segment. Nature 313:310-313.

Field GD, Sampath AP, Rieke F (2005) Retinal processing near absolute threshold: from behavior to mechanism. Annu Rev Physiol 67:491-514.

Filizola M (2010) Increasingly accurate dynamic molecular models of G-protein coupled receptor oligomers: Panacea or Pandora's box for novel drug discovery? Life Sci 86:590-597.

Foord SM, Bonner TI, Neubig RR, Rosser EM, Pin JP, Davenport AP, Spedding M, Harmar AJ (2005) International Union of Pharmacology. XLVI. G proteincoupled receptor list. Pharmacol Rev 57:279-288.

Franze K, Grosche J, Skatchkov SN, Schinkinger S, Foja C, Schild D, Uckermann O, Travis K, Reichenbach A, Guck J (2007) Muller cells are living optical fibers in the vertebrate retina. Proc Natl Acad Sci U S A 104:8287-8292.

Fredriksson R, Lagerstrom MC, Lundin LG, Schioth HB (2003) The G-proteincoupled receptors in the human genome form five main families. Phylogenetic analysis, paralogon groups, and fingerprints. Mol Pharmacol 63:1256-1272.

Fried SI, Munch TA, Werblin FS (2005) Directional selectivity is formed at multiple levels by laterally offset inhibition in the rabbit retina. Neuron 46:117-127.

Galandrin S, Oligny-Longpre G, Bouvier M (2007) The evasive nature of drug efficacy: implications for drug discovery. Trends in pharmacological sciences 28:423-430.

Ghosh KK, Bujan S, Haverkamp S, Feigenspan A, Wassle H (2004) Types of bipolar cells in the mouse retina. J Comp Neurol 469:70-82.

Gold SJ, Ni YG, Dohlman HG, Nestler EJ (1997) Regulators of G-protein signaling (RGS) proteins: region-specific expression of nine subtypes in rat brain. J Neurosci 17:8024-8037.

Gooley JJ, Lu J, Chou TC, Scammell TE, Saper CB (2001) Melanopsin in cells of origin of the retinohypothalamic tract. Nat Neurosci 4:1165.

Granit R (1933) The components of the retinal action potential in mammals and their relation to the discharge in the optic nerve. J Physiol 77:207-239.

Gregg RG, Mukhopadhyay S, Candille SI, Ball SL, Pardue MT, McCall MA, Peachey NS (2003) Identification of the gene and the mutation responsible for the mouse nob phenotype. Invest Ophthalmol Vis Sci 44:378-384.

Gregg RG, Kamermans M, Klooster J, Lukasiewicz PD, Peachey NS, Vessey KA, McCall MA (2007) Nyctalopin expression in retinal bipolar cells restores visual function in a mouse model of complete X-linked congenital stationary night blindness. J Neurophysiol 98:3023-3033.

Gross OP, Burns ME (2010) Control of rhodopsin's active lifetime by arrestin-1 expression in mammalian rods. J Neurosci 30:3450-3457.

Gurevich L, Slaughter MM (1993) Comparison of the waveforms of the ON bipolar neuron and the b-wave of the electroretinogram. Vision Res 33:2431-2435. 
Haeseleer F (2008) Interaction and colocalization of CaBP4 and Unc119 (MRG4) in photoreceptors. Invest Ophthalmol Vis Sci 49:2366-2375.

Hamann S (2002) Molecular mechanisms of water transport in the eye. Int Rev Cytol 215:395-431.

Hanson MA, Roth CB, Jo E, Griffith MT, Scott FL, Reinhart G, Desale H, Clemons B, Cahalan SM, Schuerer SC, Sanna MG, Han GW, Kuhn P, Rosen H, Stevens RC (2012) Crystal structure of a lipid G protein-coupled receptor. Science 335:851-855.

Harrington ME (1997) The ventral lateral geniculate nucleus and the intergeniculate leaflet: interrelated structures in the visual and circadian systems. Neuroscience and biobehavioral reviews 21:705-727.

Hattar S, Liao HW, Takao M, Berson DM, Yau KW (2002) Melanopsin-containing retinal ganglion cells: architecture, projections, and intrinsic photosensitivity. Science 295:1065-1070.

Haverkamp S, Grunert U, Wassle H (2000) The cone pedicle, a complex synapse in the retina. Neuron 27:85-95.

Haverkamp S, Grunert U, Wassle H (2001) The synaptic architecture of AMPA receptors at the cone pedicle of the primate retina. J Neurosci 21:2488-2500.

Haynes LW, Yau KW (1990) Single-channel measurement from the cyclic GMPactivated conductance of catfish retinal cones. J Physiol 429:451-481.

Haynes LW, Kay AR, Yau KW (1986) Single cyclic GMP-activated channel activity in excised patches of rod outer segment membrane. Nature 321:66-70.

He W, Cowan CW, Wensel TG (1998) RGS9, a GTPase accelerator for phototransduction. Neuron 20:95-102.

Hecht S, Shlaer S, Pirenne MH (1942) Energy, Quanta, and Vision. J Gen Physiol 25:819-840.

Heidelberger R, Thoreson WB, Witkovsky P (2005) Synaptic transmission at retinal ribbon synapses. Prog Retin Eye Res 24:682-720.

Helmstaedter M, Briggman KL, Turaga SC, Jain V, Seung HS, Denk W (2013) Connectomic reconstruction of the inner plexiform layer in the mouse retina. Nature 500:168-174.

Herrmann R, Heflin SJ, Hammond T, Lee B, Wang J, Gainetdinov RR, Caron MG, Eggers ED, Frishman LJ, McCall MA, Arshavsky VY (2011) Rod vision is controlled by dopamine-dependent sensitization of rod bipolar cells by GABA. Neuron 72:101-110.

Hicks D (2011) Second sight? Ecker JL, Dumitrescu ON, Wong KY, Alam NM, Chen SK, LeGates T, Renna JM, Prusky GT, Berson DM, Hattar S (2010) Melanopsinexpressing retinal ganglion-cell photoreceptors: cellular diversity and role in pattern vision. Neuron 67:49-60. Graefes Arch Clin Exp Ophthalmol 249:313314.

Higgins DG (1994) CLUSTAL V: multiple alignment of DNA and protein sequences. Methods Mol Biol 25:307-318.

Higgs MH, Lukasiewicz PD (1999) Glutamate uptake limits synaptic excitation of retinal ganglion cells. J Neurosci 19:3691-3700.

Hirota T, Fukada Y (2004) Resetting mechanism of central and peripheral circadian clocks in mammals. Zoological science 21:359-368. 
Hollander H, Makarov F, Dreher Z, van Driel D, Chan-Ling TL, Stone J (1991) Structure of the macroglia of the retina: sharing and division of labour between astrocytes and Muller cells. J Comp Neurol 313:587-603.

Homma S, Shimada T, Hikake T, Yaginuma H (2009) Expression pattern of LRR and Ig domain-containing protein (LRRIG protein) in the early mouse embryo. Gene expression patterns : GEP 9:1-26.

Hong W, Zhu H, Potter CJ, Barsh G, Kurusu M, Zinn K, Luo L (2009) Leucine-rich repeat transmembrane proteins instruct discrete dendrite targeting in an olfactory map. Nat Neurosci 12:1542-1550.

Hood DC, Finkelstein MA, Buckingham E (1979) Psychophysical tests of models of the response function. Vision Res 19:401-406.

Hu G, Wensel TG (2002) R9AP, a membrane anchor for the photoreceptor GTPase accelerating protein, RGS9-1. Proc Natl Acad Sci U S A 99:9755-9760.

Huang L, Max M, Margolskee RF, Su H, Masland RH, Euler T (2003) G protein subunit G gamma 13 is coexpressed with $\mathrm{G}$ alpha $\mathrm{o}$, $\mathrm{G}$ beta 3 , and $\mathrm{G}$ beta 4 in retinal ON bipolar cells. J Comp Neurol 455:1-10.

Hubel DH, Wiesel TN (1959) Receptive fields of single neurones in the cat's striate cortex. J Physiol 148:574-591.

Hughes A (1971) Topographical relationships between the anatomy and physiology of the rabbit visual system. Doc Ophthalmol 30:33-159.

Hughes A (1975) A quantitative analysis of the cat retinal ganglion cell topography. J Comp Neurol 163:107-128.

Hughes A, Wieniawa-Narkiewicz E (1980) A newly identified population of presumptive microneurones in the cat retinal ganglion cell layer. Nature 284:468-470.

Hughes A, University AN, Research JCSoM (1977) The Topography of Vision in Mammals of Contrasting Life Style: Comparative Optics and Retinal Organisation: Australian National University.

Hurley JB, Spencer M, Niemi GA (1998) Rhodopsin phosphorylation and its role in photoreceptor function. Vision Res 38:1341-1352.

Hynes RO, Zhao Q (2000) The evolution of cell adhesion. J Cell Biol 150:F89-96.

Inzunza O, Bravo H, Smith RL, Angel M (1991) Topography and morphology of retinal ganglion cells in Falconiforms: a study on predatory and carrioneating birds. The Anatomical record 229:271-277.

Iyer K, Burkle L, Auerbach D, Thaminy S, Dinkel M, Engels K, Stagljar I (2005) Utilizing the split-ubiquitin membrane yeast two-hybrid system to identify protein-protein interactions of integral membrane proteins. Science's STKE : signal transduction knowledge environment 2005:pl3.

Jacobs GH, Neitz J, Deegan JF, 2nd (1991) Retinal receptors in rodents maximally sensitive to ultraviolet light. Nature 353:655-656.

Jeffrey BG, Morgans CW, Puthussery T, Wensel TG, Burke NS, Brown RL, Duvoisin RM (2010) R9AP stabilizes RGS11-G beta5 and accelerates the early light response of ON-bipolar cells. Vis Neurosci 27:9-17.

Jeon CJ, Strettoi E, Masland RH (1998) The major cell populations of the mouse retina. J Neurosci 18:8936-8946. 
Jin Y, Garner CC (2008) Molecular mechanisms of presynaptic differentiation. Annual review of cell and developmental biology 24:237-262.

Jones GJ, Fein A, MacNichol EF, Jr., Cornwall MC (1993) Visual pigment bleaching in isolated salamander retinal cones. Microspectrophotometry and light adaptation. J Gen Physiol 102:483-502.

Kaneko A (1970) Physiological and morphological identification of horizontal, bipolar and amacrine cells in goldfish retina. J Physiol 207:623-633.

Kaneko A, Saito T (1983) Ionic mechanisms underlying the responses of off-center bipolar cells in the carp retina. II. Studies on responses evoked by transretinal current stimulation. J Gen Physiol 81:603-612.

Katritch V, Cherezov V, Stevens RC (2012) Diversity and modularity of G proteincoupled receptor structures. Trends in pharmacological sciences 33:17-27.

Katz B, Miledi R (1967) A study of synaptic transmission in the absence of nerve impulses. J Physiol 192:407-436.

Kennedy MJ, Dunn FA, Hurley JB (2004) Visual pigment phosphorylation but not transducin translocation can contribute to light adaptation in zebrafish cones. Neuron 41:915-928.

Kim DS, Ross SE, Trimarchi JM, Aach J, Greenberg ME, Cepko CL (2008) Identification of molecular markers of bipolar cells in the murine retina. J Comp Neurol 507:1795-1810.

Kim JM, Payne JF, Yan J, Barnes CS (2012) Negative electroretinograms in the pediatric and adult population. Doc Ophthalmol 124:41-48.

Klaassen LJ, Sun Z, Steijaert MN, Bolte P, Fahrenfort I, Sjoerdsma T, Klooster J, Claassen Y, Shields CR, Ten Eikelder HM, Janssen-Bienhold U, Zoidl G, McMahon DG, Kamermans M (2011) Synaptic transmission from horizontal cells to cones is impaired by loss of connexin hemichannels. PLoS biology 9:e1001107.

Klooster J, van Genderen MM, Yu M, Florijn RJ, Riemslag FC, Bergen AA, Gregg RG, Peachey NS, Kamermans M (2013) Ultrastructural Localization of GPR179 and the Impact of Mutant Forms on Retinal Function in CSNB1 Patients and a Mouse Model. Invest Ophthalmol Vis Sci 54:6973-6981.

Ko J (2012) The leucine-rich repeat superfamily of synaptic adhesion molecules: LRRTMs and Slitrks. Molecules and cells 34:335-340.

Kobe B, Deisenhofer J (1993) Crystal structure of porcine ribonuclease inhibitor, a protein with leucine-rich repeats. Nature 366:751-756.

Kobe B, Deisenhofer J (1994) The leucine-rich repeat: a versatile binding motif. Trends Biochem Sci 19:415-421.

Koelle MR, Horvitz HR (1996) EGL-10 regulates G protein signaling in the C. elegans nervous system and shares a conserved domain with many mammalian proteins. Cell 84:115-125.

Koh AH, Hogg CR, Holder GE (2001) The incidence of negative ERG in clinical practice. Doc Ophthalmol 102:19-30.

Koike C, Numata T, Ueda H, Mori Y, Furukawa T (2010a) TRPM1: A vertebrate TRP channel responsible for retinal ON bipolar function. Cell Calcium 48:95-101.

Koike C, Obara T, Uriu Y, Numata T, Sanuki R, Miyata K, Koyasu T, Ueno S, Funabiki K, Tani A, Ueda H, Kondo M, Mori Y, Tachibana M, Furukawa T (2010b) 
TRPM1 is a component of the retinal ON bipolar cell transduction channel in the mGluR6 cascade. Proc Natl Acad Sci U S A 107:332-337.

Kolb $H$ (1970) Organization of the outer plexiform layer of the primate retina: electron microscopy of Golgi-impregnated cells. Philosophical transactions of the Royal Society of London Series B, Biological sciences 258:261-283.

Kolb $H$ (1979) The inner plexiform layer in the retina of the cat: electron microscopic observations. J Neurocytol 8:295-329.

Kolb H, Famiglietti EV (1974) Rod and cone pathways in the inner plexiform layer of cat retina. Science 186:47-49.

Kolb H, Nelson R (1981) Amacrine cells of the cat retina. Vision Res 21:1625-1633.

Kolb H, Nelson R (1983) Rod pathways in the retina of the cat. Vision Res 23:301312.

Kolb H, Dekorver L (1991) Midget ganglion cells of the parafovea of the human retina: a study by electron microscopy and serial section reconstructions. J Comp Neurol 303:617-636.

Kolb H, Mariani A, Gallego A (1980) A second type of horizontal cell in the monkey retina. J Comp Neurol 189:31-44.

Kolb H, Linberg KA, Fisher SK (1992) Neurons of the human retina: a Golgi study. J Comp Neurol 318:147-187.

Kolb H, Fernandez E, Schouten J, Ahnelt P, Linberg KA, Fisher SK (1994) Are there three types of horizontal cell in the human retina? J Comp Neurol 343:370386.

Krispel CM, Chen D, Melling N, Chen YJ, Martemyanov KA, Quillinan N, Arshavsky VY, Wensel TG, Chen CK, Burns ME (2006) RGS expression rate-limits recovery of rod photoresponses. Neuron 51:409-416.

Lagerstrom MC, Schioth HB (2008) Structural diversity of G protein-coupled receptors and significance for drug discovery. Nature reviews Drug discovery 7:339-357.

Lamb TD (2009) Evolution of vertebrate retinal photoreception. Philosophical transactions of the Royal Society of London Series B, Biological sciences 364:2911-2924.

Lamb TD, Collin SP, Pugh EN, Jr. (2007) Evolution of the vertebrate eye: opsins, photoreceptors, retina and eye cup. Nat Rev Neurosci 8:960-976.

Latek D, Modzelewska A, Trzaskowski B, Palczewski K, Filipek S (2012) G proteincoupled receptors--recent advances. Acta biochimica Polonica 59:515-529.

Lauren J, Airaksinen MS, Saarma M, Timmusk T (2003) A novel gene family encoding leucine-rich repeat transmembrane proteins differentially expressed in the nervous system. Genomics 81:411-421.

Lebon G, Warne T, Edwards PC, Bennett K, Langmead CJ, Leslie AG, Tate CG (2011) Agonist-bound adenosine A2A receptor structures reveal common features of GPCR activation. Nature 474:521-525.

Lennie P (1984) Recent developments in the physiology of color vision. Trends in Neurosciences 7:243-248.

Letunic I, Doerks T, Bork P (2012) SMART 7: recent updates to the protein domain annotation resource. Nucleic Acids Res 40:D302-305. 
Lin B, Masland RH (2006) Populations of wide-field amacrine cells in the mouse retina. J Comp Neurol 499:797-809.

Linhoff MW, Lauren J, Cassidy RM, Dobie FA, Takahashi H, Nygaard HB, Airaksinen MS, Strittmatter SM, Craig AM (2009) An unbiased expression screen for synaptogenic proteins identifies the LRRTM protein family as synaptic organizers. Neuron 61:734-749.

Lolley RN, Farber DB (1976) Abnormal guanosine 3', 5'-monophosphate during photoreceptor degeneration in the inherited retinal disorder of $\mathrm{C} 3 \mathrm{H} / \mathrm{HeJ}$ mice. Annals of ophthalmology 8:469-473.

Lugnier C (2006) Cyclic nucleotide phosphodiesterase (PDE) superfamily: a new target for the development of specific therapeutic agents. Pharmacology \& therapeutics 109:366-398.

Lynch MA (2009) The multifaceted profile of activated microglia. Molecular neurobiology 40:139-156.

MacNeil MA, Masland RH (1998) Extreme diversity among amacrine cells: implications for function. Neuron 20:971-982.

MacNeil MA, Heussy JK, Dacheux RF, Raviola E, Masland RH (1999) The shapes and numbers of amacrine cells: matching of photofilled with Golgi-stained cells in the rabbit retina and comparison with other mammalian species. J Comp Neurol 413:305-326.

MacNeil MA, Heussy JK, Dacheux RF, Raviola E, Masland RH (2004) The population of bipolar cells in the rabbit retina. J Comp Neurol 472:73-86.

Maddox DM, Vessey KA, Yarbrough GL, Invergo BM, Cantrell DR, Inayat S, Balannik V, Hicks WL, Hawes NL, Byers S, Smith RS, Hurd R, Howell D, Gregg RG, Chang B, Naggert JK, Troy JB, Pinto LH, Nishina PM, McCall MA (2008) Allelic variance between GRM6 mutants, Grm6nob3 and Grm6nob4 results in differences in retinal ganglion cell visual responses. J Physiol 586:4409-4424.

Mangel SC (1991) Analysis of the horizontal cell contribution to the receptive field surround of ganglion cells in the rabbit retina. J Physiol 442:211-234.

Mangel SC, Miller RF (1987) Horizontal cells contribute to the receptive field surround of ganglion cells in the rabbit retina. Brain Res 414:182-186.

Marc RE, Liu WL, Kalloniatis M, Raiguel SF, van Haesendonck E (1990) Patterns of glutamate immunoreactivity in the goldfish retina. J Neurosci 10:4006-4034.

Margeta-Mitrovic M, Jan YN, Jan LY (2000) A trafficking checkpoint controls GABA(B) receptor heterodimerization. Neuron 27:97-106.

Mariani AP (1990) Amacrine cells of the rhesus monkey retina. J Comp Neurol 301:382-400.

Martemyanov KA, Yoo PJ, Skiba NP, Arshavsky VY (2005) R7BP, a novel neuronal protein interacting with RGS proteins of the R7 family. J Biol Chem 280:51335136.

Masland RH (1986) The functional architecture of the retina. Sci Am 255:102-111.

Masland RH (2001a) Neuronal diversity in the retina. Curr Opin Neurobiol 11:431436.

Masland RH (2001b) The fundamental plan of the retina. Nat Neurosci 4:877-886. Masland RH (2011) Cell populations of the retina: the Proctor lecture. Invest Ophthalmol Vis Sci 52:4581-4591. 
Masland RH (2012) The neuronal organization of the retina. Neuron 76:266-280.

Masu M, Iwakabe H, Tagawa Y, Miyoshi T, Yamashita M, Fukuda Y, Sasaki H, Hiroi K, Nakamura Y, Shigemoto R, et al. (1995) Specific deficit of the ON response in visual transmission by targeted disruption of the mGluR6 gene. Cell 80:757765.

Matsui K, Hosoi N, Tachibana M (1999) Active role of glutamate uptake in the synaptic transmission from retinal nonspiking neurons. J Neurosci 19:67556766.

Matsushima N, Tachi N, Kuroki Y, Enkhbayar P, Osaki M, Kamiya M, Kretsinger RH (2005) Structural analysis of leucine-rich-repeat variants in proteins associated with human diseases. Cell Mol Life Sci 62:2771-2791.

Matthews G (1987) Single-channel recordings demonstrate that cGMP opens the light-sensitive ion channel of the rod photoreceptor. Proc Natl Acad Sci U S A 84:299-302.

Matthews HR, Sampath AP (2010) Photopigment quenching is Ca2+ dependent and controls response duration in salamander L-cone photoreceptors. J Gen Physiol 135:355-366.

McAllister AK (2007) Dynamic aspects of CNS synapse formation. Annu Rev Neurosci 30:425-450.

McCall MA, Gregg RG (2008) Comparisons of structural and functional abnormalities in mouse b-wave mutants. J Physiol 586:4385-4392.

Mendez A, Burns ME, Roca A, Lem J, Wu LW, Simon MI, Baylor DA, Chen J (2000) Rapid and reproducible deactivation of rhodopsin requires multiple phosphorylation sites. Neuron 28:153-164.

Merigan WH, Maunsell JHR (1993) HOW PARALLEL ARE THE PRIMATE VISUAL PATHWAYS. Annual Review of Neuroscience 16:369-402.

Middleton WE, Mayo EG (1952) The appearance of colors in twilight. Journal of the Optical Society of America 42:116-121.

Milligan G (2006) G-protein-coupled receptor heterodimers: pharmacology, function and relevance to drug discovery. Drug discovery today 11:541-549.

Mills SL, Massey SC (1995) Differential properties of two gap junctional pathways made by AII amacrine cells. Nature 377:734-737.

Miyake Y, Horiguchi M, Ota I, Shiroyama N (1987) Characteristic ERG-flicker anomaly in incomplete congenital stationary night blindness. Invest Ophthalmol Vis Sci 28:1816-1823.

Miyake Y, Yagasaki K, Horiguchi M, Kawase Y, Kanda T (1986) Congenital stationary night blindness with negative electroretinogram. A new classification. Arch Ophthalmol 104:1013-1020.

Monnier C, Tu H, Bourrier E, Vol C, Lamarque L, Trinquet E, Pin JP, Rondard P (2011) Trans-activation between 7TM domains: implication in heterodimeric GABAB receptor activation. Embo J 30:32-42.

Moore BA, Kamilar JM, Collin SP, Bininda-Emonds OR, Dominy NJ, Hall MI, Heesy CP, Johnsen S, Lisney TJ, Loew ER, Moritz G, Nava SS, Warrant E, Yopak KE, Fernandez-Juricic E (2012) A novel method for comparative analysis of retinal specialization traits from topographic maps. J Vis 12:13. 
Morgan J, Wong R (1995) Development of Cell Types and Synaptic Connections in the Retina. In: Webvision: The Organization of the Retina and Visual System (Kolb H, Fernandez E, Nelson R, eds). Salt Lake City (UT).

Morgans CW, Brown RL, Duvoisin RM (2010) TRPM1: the endpoint of the mGluR6 signal transduction cascade in retinal ON-bipolar cells. Bioessays 32:609614.

Morgans CW, Weiwei L, Wensel TG, Brown RL, Perez-Leon JA, Bearnot B, Duvoisin RM (2007) Gbeta5-RGS complexes co-localize with mGluR6 in retinal ONbipolar cells. Eur J Neurosci 26:2899-2905.

Morgans CW, Zhang J, Jeffrey BG, Nelson SM, Burke NS, Duvoisin RM, Brown RL (2009) TRPM1 is required for the depolarizing light response in retinal ONbipolar cells. Proc Natl Acad Sci U S A 106:19174-19178.

Morin LP, Blanchard JH, Provencio I (2003) Retinal ganglion cell projections to the hamster suprachiasmatic nucleus, intergeniculate leaflet, and visual midbrain: bifurcation and melanopsin immunoreactivity. J Comp Neurol 465:401-416.

Muller DJ, Wu N, Palczewski K (2008) Vertebrate membrane proteins: structure, function, and insights from biophysical approaches. Pharmacol Rev 60:43-78.

Munch TA, da Silveira RA, Siegert S, Viney TJ, Awatramani GB, Roska B (2009) Approach sensitivity in the retina processed by a multifunctional neural circuit. Nat Neurosci 12:1308-1316.

Murphy GJ, Rieke F (2008) Signals and noise in an inhibitory interneuron diverge to control activity in nearby retinal ganglion cells. Nat Neurosci 11:318-326.

Nakamura M, Sanuki R, Yasuma TR, Onishi A, Nishiguchi KM, Koike C, Kadowaki M, Kondo M, Miyake Y, Furukawa T (2010) TRPM1 mutations are associated with the complete form of congenital stationary night blindness. Mol Vis 16:425-437.

Nawy S, Jahr CE (1991) cGMP-gated conductance in retinal bipolar cells is suppressed by the photoreceptor transmitter. Neuron 7:677-683.

Nelson R (1977) Cat cones have rod input: a comparison of the response properties of cones and horizontal cell bodies in the retina of the cat. J Comp Neurol 172:109-135.

Nelson R, Kolb H (1985) A17: a broad-field amacrine cell in the rod system of the cat retina. J Neurophysiol 54:592-614.

Nelson R, Famiglietti EV, Jr., Kolb H (1978) Intracellular staining reveals different levels of stratification for on- and off-center ganglion cells in cat retina. J Neurophysiol 41:472-483.

Nikonov SS, Kholodenko R, Lem J, Pugh EN, Jr. (2006) Physiological features of the Sand M-cone photoreceptors of wild-type mice from single-cell recordings. J Gen Physiol 127:359-374.

Noell WK (1954) The origin of the electroretinogram. Am J Ophthalmol 38:78-90.

O'Connor E, Eisenhaber B, Dalley J, Wang T, Missen C, Bulleid N, Bishop PN, Trump D (2005) Species specific membrane anchoring of nyctalopin, a small leucinerich repeat protein. Hum Mol Genet 14:1877-1887. 
Okawa H, Pahlberg J, Rieke F, Birnbaumer L, Sampath AP (2010) Coordinated control of sensitivity by two splice variants of Galpha(o) in retinal ON bipolar cells. J Gen Physiol 136:443-454.

Orlandi C, Cao Y, Martemyanov K (2013) Orphan receptor GPR179 forms macromolecular complexes with components of metabotropic signaling cascade in retina ON-bipolar neurons. Invest Ophthalmol Vis Sci.

Orlandi C, Posokhova E, Masuho I, Ray TA, Hasan N, Gregg RG, Martemyanov KA (2012) GPR158/179 regulate G protein signaling by controlling localization and activity of the RGS7 complexes. J Cell Biol 197:711-719.

Osterberg G (1937) Topography of the layer of rods and cones in the human retina. Journal of the American Medical Association 108:232-232.

Oyster CW, Barlow HB (1967) Direction-selective units in rabbit retina: distribution of preferred directions. Science 155:841-842.

Pagano A, Rovelli G, Mosbacher J, Lohmann T, Duthey B, Stauffer D, Ristig D, Schuler V, Meigel I, Lampert C, Stein T, Prezeau L, Blahos J, Pin J, Froestl W, Kuhn R, Heid J, Kaupmann K, Bettler B (2001) C-terminal interaction is essential for surface trafficking but not for heteromeric assembly of GABA(b) receptors. J Neurosci 21:1189-1202.

Palczewski K, Kumasaka T, Hori T, Behnke CA, Motoshima H, Fox BA, Le Trong I, Teller DC, Okada T, Stenkamp RE, Yamamoto M, Miyano M (2000) Crystal structure of rhodopsin: A G protein-coupled receptor. Science 289:739-745.

Pardue M, Ball S, Candille S, McCall M, Gregg R, Peachey N (2001) nob: A Mouse Model of CSNB1. In: New Insights Into Retinal Degenerative Diseases (Anderson R, LaVail M, Hollyfield J, eds), pp 319-328: Springer US.

Pardue MT, McCall MA, LaVail MM, Gregg RG, Peachey NS (1998) A naturally occurring mouse model of X-linked congenital stationary night blindness. Invest Ophthalmol Vis Sci 39:2443-2449.

Park JH, Scheerer P, Hofmann KP, Choe HW, Ernst OP (2008) Crystal structure of the ligand-free G-protein-coupled receptor opsin. Nature 454:183-187.

Parthier C, Reedtz-Runge S, Rudolph R, Stubbs MT (2009) Passing the baton in class B GPCRs: peptide hormone activation via helix induction? Trends Biochem Sci 34:303-310.

Peachey NS, Ball SL (2003) Electrophysiological analysis of visual function in mutant mice. Doc Ophthalmol 107:13-36.

Peachey NS, Pearring JN, Bojang P, Jr., Hirschtritt ME, Sturgill-Short G, Ray TA, Furukawa T, Koike C, Goldberg AF, Shen Y, McCall MA, Nawy S, Nishina PM, Gregg RG (2012a) Depolarizing bipolar cell dysfunction due to a Trpm1 point mutation. J Neurophysiol 108:2442-2451.

Peachey NS et al. (2012b) GPR179 is required for depolarizing bipolar cell function and is mutated in autosomal-recessive complete congenital stationary night blindness. Am J Hum Genet 90:331-339.

Pearring JN, Bojang P, Jr., Shen Y, Koike C, Furukawa T, Nawy S, Gregg RG (2011) A role for nyctalopin, a small leucine-rich repeat protein, in localizing the TRP melastatin 1 channel to retinal depolarizing bipolar cell dendrites. J Neurosci 31:10060-10066. 
Peichl L, Gonzalez-Soriano J (1994) Morphological types of horizontal cell in rodent retinae: a comparison of rat, mouse, gerbil, and guinea pig. Vis Neurosci 11:501-517.

Penn RD, Hagins WA (1969) Signal transmission along retinal rods and the origin of the electroretinographic a-wave. Nature 223:201-204.

Perry VH, Oehler R, Cowey A (1984) Retinal ganglion cells that project to the dorsal lateral geniculate nucleus in the macaque monkey. Neuroscience 12:11011123.

Petrides A, Trexler EB (2008) Differential output of the high-sensitivity rod photoreceptor: AII amacrine pathway. J Comp Neurol 507:1653-1662.

Pin JP, Galvez T, Prezeau L (2003) Evolution, structure, and activation mechanism of family 3/C G-protein-coupled receptors. Pharmacology \& therapeutics 98:325-354.

Pinto LH et al. (2007) Generation, identification and functional characterization of the nob4 mutation of Grm6 in the mouse. Vis Neurosci 24:111-123.

Pittler SJ, Baehr W (1991) Identification of a nonsense mutation in the rod photoreceptor cGMP phosphodiesterase beta-subunit gene of the rd mouse. Proc Natl Acad Sci U S A 88:8322-8326.

Porter MY, Koelle MR (2010) RSBP-1 is a membrane-targeting subunit required by the Galpha(q)-specific but not the Galpha(o)-specific R7 regulator of G protein signaling in Caenorhabditis elegans. Mol Biol Cell 21:232-243.

Pow DV, Crook DK (1996) Direct immunocytochemical evidence for the transfer of glutamine from glial cells to neurons: use of specific antibodies directed against the d-stereoisomers of glutamate and glutamine. Neuroscience 70:295-302.

Protti DA, Llano I (1998) Calcium currents and calcium signaling in rod bipolar cells of rat retinal slices. J Neurosci 18:3715-3724.

Provis JM (1979) The distribution and size of ganglion cells in the regina of the pigmented rabbit: a quantitative analysis. J Comp Neurol 185:121-137.

Provis JM, Diaz CM, Dreher B (1998) Ontogeny of the primate fovea: a central issue in retinal development. Progress in neurobiology 54:549-580.

Puller C, Ivanova E, Euler T, Haverkamp S, Schubert T (2013) OFF bipolar cells express distinct types of dendritic glutamate receptors in the mouse retina. Neuroscience 243:136-148.

Pusch CM, Zeitz C, Brandau O, Pesch K, Achatz H, Feil S, Scharfe C, Maurer J, Jacobi FK, Pinckers A, Andreasson S, Hardcastle A, Wissinger B, Berger W, Meindl A (2000) The complete form of X-linked congenital stationary night blindness is caused by mutations in a gene encoding a leucine-rich repeat protein. Nat Genet 26:324-327.

Qin N, Baehr W (1994) Expression and mutagenesis of mouse rod photoreceptor cGMP phosphodiesterase. J Biol Chem 269:3265-3271.

Rao A, Dallman R, Henderson S, Chen CK (2007) Gbeta5 is required for normal light responses and morphology of retinal ON-bipolar cells. J Neurosci 27:1419914204. 
Rapti G, Richmond J, Bessereau JL (2011) A single immunoglobulin-domain protein required for clustering acetylcholine receptors in C. elegans. Embo J 30:706718.

Rasmussen SG, DeVree BT, Zou Y, Kruse AC, Chung KY, Kobilka TS, Thian FS, Chae PS, Pardon E, Calinski D, Mathiesen JM, Shah ST, Lyons JA, Caffrey M, Gellman SH, Steyaert J, Skiniotis G, Weis WI, Sunahara RK, Kobilka BK (2011) Crystal structure of the beta2 adrenergic receptor-Gs protein complex. Nature 477:549-555.

Raviola E, Gilula NB (1973) Gap junctions between photoreceptor cells in the vertebrate retina. Proc Natl Acad Sci U S A 70:1677-1681.

Raviola E, Dacheux RF (1987) Excitatory dyad synapse in rabbit retina. Proc Natl Acad Sci U S A 84:7324-7328.

Ray K, Hauschild BC, Steinbach PJ, Goldsmith PK, Hauache O, Spiegel AM (1999) Identification of the cysteine residues in the amino-terminal extracellular domain of the human $\mathrm{Ca}(2+)$ receptor critical for dimerization. Implications for function of monomeric $\mathrm{Ca}(2+)$ receptor. J Biol Chem 274:27642-27650.

Reichenbach A, Robinson SR (1995) Phylogenetic constraints on retinal organisation and development. Progress in Retinal and Eye Research 15:139171.

Reichenbach A, Bringmann A (2013) New functions of Muller cells. Glia 61:651-678.

Renner AB, Kellner U, Cropp E, Foerster MH (2006) Dysfunction of transmission in the inner retina: incidence and clinical causes of negative electroretinogram. Graefes Arch Clin Exp Ophthalmol 244:1467-1473.

Ridet JL, Malhotra SK, Privat A, Gage FH (1997) Reactive astrocytes: cellular and molecular cues to biological function. Trends Neurosci 20:570-577.

Riggs LA (1954) Electroretinography in cases of night blindness. Am J Ophthalmol 38:70-78.

Rockhill RL, Daly FJ, MacNeil MA, Brown SP, Masland RH (2002) The diversity of ganglion cells in a mammalian retina. J Neurosci 22:3831-3843.

Rodieck R (1973) The Vertebrate Retina: Principles of Structure and Function. 1973. In: WH Freeman San Francisco.

Rohlich P, van Veen T, Szel A (1994) Two different visual pigments in one retinal cone cell. Neuron 13:1159-1166.

Rondard P, Goudet C, Kniazeff J, Pin JP, Prezeau L (2011) The complexity of their activation mechanism opens new possibilities for the modulation of mGlu and GABAB class C G protein-coupled receptors. Neuropharmacology 60:8292.

Rondard P, Huang S, Monnier C, Tu H, Blanchard B, Oueslati N, Malhaire F, Li Y, Trinquet E, Labesse G, Pin JP, Liu J (2008) Functioning of the dimeric GABA(B) receptor extracellular domain revealed by glycan wedge scanning. Embo J 27:1321-1332.

Roska B, Werblin F (2001) Vertical interactions across ten parallel, stacked representations in the mammalian retina. Nature 410:583-587.

Ross EM, Wilkie TM (2000) GTPase-activating proteins for heterotrimeric G proteins: regulators of G protein signaling (RGS) and RGS-like proteins. Annu Rev Biochem 69:795-827. 
Saito T, Kaneko A (1983) Ionic mechanisms underlying the responses of off-center bipolar cells in the carp retina. I. Studies on responses evoked by light. J Gen Physiol 81:589-601.

Sakitt B (1972) Counting every quantum. J Physiol 223:131-150.

Salon JA, Lodowski DT, Palczewski K (2011) The significance of G protein-coupled receptor crystallography for drug discovery. Pharmacol Rev 63:901-937.

Sand A, Schmidt TM, Kofuji P (2012) Diverse types of ganglion cell photoreceptors in the mammalian retina. Prog Retin Eye Res 31:287-302.

Scheerer P, Park JH, Hildebrand PW, Kim YJ, Krauss N, Choe HW, Hofmann KP, Ernst OP (2008) Crystal structure of opsin in its G-protein-interacting conformation. Nature 455:497-502.

Schein SJ (1988) Anatomy of macaque fovea and spatial densities of neurons in foveal representation. J Comp Neurol 269:479-505.

Schiller PH, Logothetis NK (1990) THE COLOR-OPPONENT AND BROAD-BAND CHANNELS OF THE PRIMATE VISUAL-SYSTEM. Trends in Neurosciences 13:392-398.

Schmitz F (2009) The making of synaptic ribbons: how they are built and what they do. The Neuroscientist : a review journal bringing neurobiology, neurology and psychiatry 15:611-624.

Schmitz F, Konigstorfer A, Sudhof TC (2000) RIBEYE, a component of synaptic ribbons: a protein's journey through evolution provides insight into synaptic ribbon function. Neuron 28:857-872.

Schmitz Y, Witkovsky P (1997) Dependence of photoreceptor glutamate release on a dihydropyridine-sensitive calcium channel. Neuroscience 78:1209-1216.

Schneeweis DM, Schnapf JL (1995) Photovoltage of rods and cones in the macaque retina. Science 268:1053-1056.

Schultze M (1866) Zur Anatomie und Physiologie der Retina. Archiv f mikrosk Anatomie 2:175-286.

Schutte M, Werner P (1998) Redistribution of glutathione in the ischemic rat retina. Neurosci Lett 246:53-56.

Selander-Sunnerhagen M, Ullner M, Persson E, Teleman O, Stenflo J, Drakenberg T (1992) How an epidermal growth factor (EGF)-like domain binds calcium. High resolution NMR structure of the calcium form of the NH2-terminal EGFlike domain in coagulation factor X. Journal of Biological Chemistry 267:19642-19649.

Sgourakis NG, Bagos PG, Hamodrakas SJ (2005a) Prediction of the coupling specificity of GPCRs to four families of G-proteins using hidden Markov models and artificial neural networks. Bioinformatics 21:4101-4106.

Sgourakis NG, Bagos PG, Papasaikas PK, Hamodrakas SJ (2005b) A method for the prediction of GPCRs coupling specificity to G-proteins using refined profile Hidden Markov Models. BMC bioinformatics 6:104.

Shen Y, Rampino MA, Carroll RC, Nawy S (2012) G-protein-mediated inhibition of the Trp channel TRPM1 requires the Gbetagamma dimer. Proc Natl Acad Sci U S A 109:8752-8757. 
Shen Y, Heimel JA, Kamermans M, Peachey NS, Gregg RG, Nawy S (2009) A transient receptor potential-like channel mediates synaptic transmission in rod bipolar cells. J Neurosci 29:6088-6093.

Siddiqui TJ, Pancaroglu R, Kang Y, Rooyakkers A, Craig AM (2010) LRRTMs and neuroligins bind neurexins with a differential code to cooperate in glutamate synapse development. J Neurosci 30:7495-7506.

Sidman RL, Green MC (1965) Retinal Degeneration in the Mouse: Location of the Rd Locus in Linkage Group Xvii. The Journal of heredity 56:23-29.

Simossis VA, Heringa J (2005) PRALINE: a multiple sequence alignment toolbox that integrates homology-extended and secondary structure information. Nucleic Acids Res 33:W289-294.

Sjostrand FS (1953) The ultrastructure of the outer segments of rods and cones of the eye as revealed by the electron microscope. J Cell Physiol 42:15-44.

Slaughter MM, Miller RF (1981) 2-amino-4-phosphonobutyric acid: a new pharmacological tool for retina research. Science 211:182-185.

Slaughter MM, Miller RF (1983) An excitatory amino acid antagonist blocks cone input to sign-conserving second-order retinal neurons. Science 219:12301232.

Smith SJ, Augustine GJ (1988) Calcium ions, active zones and synaptic transmitter release. Trends Neurosci 11:458-464.

Snodderly DM, Auran JD, Delori FC (1984a) The macular pigment. II. Spatial distribution in primate retinas. Invest Ophthalmol Vis Sci 25:674-685.

Snodderly DM, Brown PK, Delori FC, Auran JD (1984b) The macular pigment. I. Absorbance spectra, localization, and discrimination from other yellow pigments in primate retinas. Invest Ophthalmol Vis Sci 25:660-673.

Soding J (2005) Protein homology detection by HMM-HMM comparison. Bioinformatics 21:951-960.

Soler-Llavina GJ, Arstikaitis P, Morishita W, Ahmad M, Sudhof TC, Malenka RC (2013) Leucine-rich repeat transmembrane proteins are essential for maintenance of long-term potentiation. Neuron 79:439-446.

Sparrow JR, Hicks D, Hamel CP (2010) The retinal pigment epithelium in health and disease. Current molecular medicine 10:802-823.

Standfuss J, Edwards PC, D'Antona A, Fransen M, Xie G, Oprian DD, Schertler GF (2011) The structural basis of agonist-induced activation in constitutively active rhodopsin. Nature 471:656-660.

Steinberg RH (1985) Interactions between the retinal pigment epithelium and the neural retina. Doc Ophthalmol 60:327-346.

Steinberg RH, Schmidt R, Brown KT (1970) Intracellular responses to light from cat pigment epithelium: origin of the electroretinogram c-wave. Nature 227:728730 .

Sterling P, Matthews G (2005) Structure and function of ribbon synapses. Trends Neurosci 28:20-29.

Stockman A, Sharpe LT (2006) Into the twilight zone: the complexities of mesopic vision and luminous efficiency. Ophthalmic and Physiological Optics 26:225239. 
Stockton RA, Slaughter MM (1989) B-wave of the electroretinogram. A reflection of ON bipolar cell activity. J Gen Physiol 93:101-122.

Stone J (1978) The number and distribution of ganglion cells in the cat's retina. J Comp Neurol 180:753-771.

Stone J (1983) Parallel processing in the visual system: the classification of retinal ganglion cells and its impact on the neurobiology of vision: Plenum Press.

Strauss $O$ (2005) The retinal pigment epithelium in visual function. Physiol Rev 85:845-881.

Strekalova T, Sun M, Sibbe M, Evers M, Dityatev A, Gass P, Schachner M (2002) Fibronectin domains of extracellular matrix molecule tenascin- $\mathrm{C}$ modulate hippocampal learning and synaptic plasticity. Molecular and cellular neurosciences 21:173-187.

Strettoi E, Masland RH (1996) The number of unidentified amacrine cells in the mammalian retina. Proc Natl Acad Sci U S A 93:14906-14911.

Strettoi E, Raviola E, Dacheux RF (1992) Synaptic connections of the narrow-field, bistratified rod amacrine cell (AII) in the rabbit retina. J Comp Neurol 325:152-168.

Strettoi E, Dacheux RF, Raviola E (1994) Cone bipolar cells as interneurons in the rod pathway of the rabbit retina. J Comp Neurol 347:139-149.

Strom TM, Nyakatura G, Apfelstedt-Sylla E, Hellebrand H, Lorenz B, Weber BH, Wutz K, Gutwillinger N, Ruther K, Drescher B, Sauer C, Zrenner E, Meitinger T, Rosenthal A, Meindl A (1998) An L-type calcium-channel gene mutated in incomplete X-linked congenital stationary night blindness. Nat Genet 19:260263.

Stryer L (1991) Visual excitation and recovery. J Biol Chem 266:10711-10714.

Sun L, Han X, He S (2011) Direction-selective circuitry in rat retina develops independently of GABAergic, cholinergic and action potential activity. PLoS One 6:e19477.

Sun Z, Risner ML, van Asselt JB, Zhang DQ, Kamermans M, McMahon DG (2012) Physiological and molecular characterization of connexin hemichannels in zebrafish retinal horizontal cells. J Neurophysiol 107:2624-2632.

Tagawa Y, Sawai H, Ueda Y, Tauchi M, Nakanishi S (1999) Immunohistological studies of metabotropic glutamate receptor subtype 6-deficient mice show no abnormality of retinal cell organization and ganglion cell maturation. J Neurosci 19:2568-2579.

Talbot CM, Marshall JN (2011) The retinal topography of three species of coleoid cephalopod: significance for perception of polarized light. Philosophical transactions of the Royal Society of London Series B, Biological sciences 366:724-733.

Taylor BA, Navin A, Phillips SJ (1994) PCR-amplification of simple sequence repeat variants from pooled DNA samples for rapidly mapping new mutations of the mouse. Genomics 21:626-632.

Thompson DA, Gal A (2003) Genetic defects in vitamin A metabolism of the retinal pigment epithelium. Developments in ophthalmology 37:141-154.

Thoreson WB (2007) Kinetics of synaptic transmission at ribbon synapses of rods and cones. Molecular neurobiology 36:205-223. 
Thoreson WB, Rabl K, Townes-Anderson E, Heidelberger R (2004) A highly Ca2+sensitive pool of vesicles contributes to linearity at the rod photoreceptor ribbon synapse. Neuron 42:595-605.

Trejo LJ, Cicerone CM (1984) Cells in the pretectal olivary nucleus are in the pathway for the direct light reflex of the pupil in the rat. Brain Res 300:49-62.

Trexler EB, Li W, Massey SC (2005) Simultaneous contribution of two rod pathways to AII amacrine and cone bipolar cell light responses. J Neurophysiol 93:1476-1485.

Trifonov IU (1968a) [Study of synaptic transmission between photoreceptor and horizontal cell by electric stimulations of the retina]. Biofizika 13:809-817.

Trifonov YA (1968b) Study of synaptic transmission between photoreceptor and horizontal cell by means of electrical stimulation of the retina. [Study of synaptic transmission between photoreceptor and horizontal cell by means of electrical stimulation of the retina.]. Biofizika 13:809-817.

Tsukamoto Y, Morigiwa K, Ueda M, Sterling P (2001) Microcircuits for night vision in mouse retina. J Neurosci 21:8616-8623.

Untergasser A, Cutcutache I, Koressaar T, Ye J, Faircloth BC, Remm M, Rozen SG (2012) Primer3--new capabilities and interfaces. Nucleic Acids Res 40:e115.

Valois R (1973) Central Mechanisms of Color Vision. In: Central Processing of Visual Information A: Integrative Functions and Comparative Data (Jung R, ed), pp 209-253: Springer Berlin Heidelberg.

Van Der Velden HA (1946) The number of quanta necessary for the perception of light of the human eye. Ophthalmologica Journal international d'ophtalmologie International journal of ophthalmology Zeitschrift fur Augenheilkunde 111:321-331.

van Genderen MM, Bijveld MM, Claassen YB, Florijn RJ, Pearring JN, Meire FM, McCall MA, Riemslag FC, Gregg RG, Bergen AA, Kamermans M (2009) Mutations in TRPM1 are a common cause of complete congenital stationary night blindness. Am J Hum Genet 85:730-736.

VanLeeuwen M, Fahrenfort I, Sjoerdsma T, Numan R, Kamermans M (2009) Lateral gain control in the outer retina leads to potentiation of center responses of retinal neurons. J Neurosci 29:6358-6366.

Venkatakrishnan AJ, Deupi X, Lebon G, Tate CG, Schertler GF, Babu MM (2013) Molecular signatures of G-protein-coupled receptors. Nature 494:185-194.

Veruki ML, Hartveit E (2002) AII (Rod) amacrine cells form a network of electrically coupled interneurons in the mammalian retina. Neuron 33:935-946.

Wa"ssle H, Gru"nert U, Martin PR, Boycotts BB (1994) Immunocytochemical characterization and spatial distribution of midget bipolar cells in the macaque monkey retina. Vision Research 34:561-579.

Waites CL, Craig AM, Garner CC (2005) Mechanisms of vertebrate synaptogenesis. Annu Rev Neurosci 28:251-274.

Wald G (1968) Molecular basis of visual excitation. Science 162:230-239.

Wassle H (2004) Parallel processing in the mammalian retina. Nat Rev Neurosci 5:747-757.

Wassle H, Boycott BB (1991) Functional architecture of the mammalian retina. Physiol Rev 71:447-480. 
Wassle H, Grunert U, Rohrenbeck J, Boycott BB (1989) Cortical magnification factor and the ganglion cell density of the primate retina. Nature 341:643-646.

Wassle H, Puller C, Muller F, Haverkamp S (2009) Cone contacts, mosaics, and territories of bipolar cells in the mouse retina. J Neurosci 29:106-117.

Werblin FS, Dowling JE (1969) Organization of the retina of the mudpuppy, Necturus maculosus. II. Intracellular recording. J Neurophysiol 32:339-355.

Whorton MR, Bokoch MP, Rasmussen SG, Huang B, Zare RN, Kobilka B, Sunahara RK (2007) A monomeric G protein-coupled receptor isolated in a high-density lipoprotein particle efficiently activates its G protein. Proc Natl Acad Sci U S A 104:7682-7687.

Wiesel TN, Hubel DH (1966) Spatial and chromatic interactions in the lateral geniculate body of the rhesus monkey. J Neurophysiol 29:1115-1156.

Woo J, Kwon SK, Choi S, Kim S, Lee JR, Dunah AW, Sheng M, Kim E (2009) Transsynaptic adhesion between NGL-3 and LAR regulates the formation of excitatory synapses. Nat Neurosci 12:428-437.

Xie H, Vucetic S, Iakoucheva LM, Oldfield CJ, Dunker AK, Uversky VN, Obradovic Z (2007) Functional anthology of intrinsic disorder. 1. Biological processes and functions of proteins with long disordered regions. Journal of proteome research 6:1882-1898.

Xu F, Wu H, Katritch V, Han GW, Jacobson KA, Gao ZG, Cherezov V, Stevens RC (2011) Structure of an agonist-bound human A2A adenosine receptor. Science 332:322-327.

Xu J, Dodd RL, Makino CL, Simon MI, Baylor DA, Chen J (1997) Prolonged photoresponses in transgenic mouse rods lacking arrestin. Nature 389:505509.

Xu Y, Dhingra A, Fina ME, Koike C, Furukawa T, Vardi N (2012) mGluR6 deletion renders the TRPM1 channel in retina inactive. J Neurophysiol 107:948-957.

Yamauchi T (2002) Molecular constituents and phosphorylation-dependent regulation of the post-synaptic density. Mass spectrometry reviews 21:266286.

Yanagawa M, Yamashita T, Shichida Y (2011) Comparative fluorescence resonance energy transfer analysis of metabotropic glutamate receptors: implications about the dimeric arrangement and rearrangement upon ligand bindings. J Biol Chem 286:22971-22981.

Yoshimura Y, Yamauchi Y, Shinkawa T, Taoka M, Donai H, Takahashi N, Isobe T, Yamauchi T (2004) Molecular constituents of the postsynaptic density fraction revealed by proteomic analysis using multidimensional liquid chromatography-tandem mass spectrometry. J Neurochem 88:759-768.

Young RW (1967) The renewal of photoreceptor cell outer segments. J Cell Biol 33:61-72.

Zeitz C, Kloeckener-Gruissem B, Forster U, Kohl S, Magyar I, Wissinger B, Matyas G, Borruat FX, Schorderet DF, Zrenner E, Munier FL, Berger W (2006) Mutations in CABP4, the gene encoding the Ca2+-binding protein 4, cause autosomal recessive night blindness. Am J Hum Genet 79:657-667.

Zeitz C, van Genderen M, Neidhardt J, Luhmann UF, Hoeben F, Forster U, Wycisk K, Matyas G, Hoyng CB, Riemslag F, Meire F, Cremers FP, Berger W (2005) 
Mutations in GRM6 cause autosomal recessive congenital stationary night blindness with a distinctive scotopic $15-\mathrm{Hz}$ flicker electroretinogram. Invest Ophthalmol Vis Sci 46:4328-4335.

Zeitz C et al. (2013a) Whole-exome sequencing identifies LRIT3 mutations as a cause of autosomal-recessive complete congenital stationary night blindness. Am J Hum Genet 92:67-75.

Zeitz C et al. (2013b) Whole-exome sequencing identifies LRIT3 mutations as a cause of autosomal-recessive complete congenital stationary night blindness. Am J Hum Genet 92:67-75.

Zhang F, Klebansky B, Fine RM, Xu H, Pronin A, Liu H, Tachdjian C, Li X (2008) Molecular mechanism for the umami taste synergism. Proc Natl Acad Sci U S A 105:20930-20934.

Zhang J, Li W, Trexler EB, Massey SC (2002) Confocal analysis of reciprocal feedback at rod bipolar terminals in the rabbit retina. J Neurosci 22:10871-10882.

Zhang J, Jeffrey BG, Morgans CW, Burke NS, Haley TL, Duvoisin RM, Brown RL (2010) RGS7 and -11 complexes accelerate the ON-bipolar cell light response. Invest Ophthalmol Vis Sci 51:1121-1129.

Zhang X, Cote RH (2005) cGMP signaling in vertebrate retinal photoreceptor cells. Frontiers in bioscience : a journal and virtual library 10:1191-1204.

Zhang X, Wensel TG, Kraft TW (2003) GTPase regulators and photoresponses in cones of the eastern chipmunk. J Neurosci 23:1287-1297.

Zimmerman AL, Baylor DA (1986) Cyclic GMP-sensitive conductance of retinal rods consists of aqueous pores. Nature 321:70-72.

Zinkernagel MS, Chinnery HR, Ong ML, Petitjean C, Voigt V, McLenachan S, McMenamin PG, Hill GR, Forrester JV, Wikstrom ME, Degli-Esposti MA (2013) Interferon $\gamma$-Dependent Migration of Microglial Cells in the Retina after Systemic Cytomegalovirus Infection. The American Journal of Pathology 182:875-885.

Zody MC et al. (2006) DNA sequence of human chromosome 17 and analysis of rearrangement in the human lineage. Nature 440:1045-1049. 


\section{APPENDIX I: GPR179 Is Required for Depolarizing Bipolar Cell Function and Is Mutated in Autosomal-Recessive Complete Congenital Stationary Night Blindness}

Elsevier License Number: 3275981045307

Peachey et al., 2012. Originally published in The American Journal of Human Genetics DOI: S0002-9297(11)00541-6

Neal S. Peachey, ${ }^{1,2,3}$ Thomas A. Ray, ${ }^{4}$ Ralph Florijn, ${ }^{5}$ Lucy B. Rowe, ${ }^{6}$ Trijntje Sjoerdsma, ${ }^{7}$ Susana Contreras-Alcantara, ${ }^{8}$ Kenkichi Baba, ${ }^{8}$ Gianluca Tosini, ${ }^{8}$ Nikita Pozdeyev, ${ }^{9}$ P. Michael luvone, ${ }^{9}$ Pasano Bojang, Jr., ${ }^{4}$ Jillian N. Pearring, ${ }^{4}$ Huibert Jan Simonsz, ${ }^{10}$ Maria van Genderen, ${ }^{11}$ David G. Birch, ${ }^{12}$ Elias I. Traboulsi, ${ }^{1,3}$ Allison Dorfman, ${ }^{13}$ Irma Lopez, ${ }^{13}$ Huanan Ren, ${ }^{13}$ Andrew F.X. Goldberg, ${ }^{14}$ Patsy M. Nishina, ${ }^{6}$ Pierre Lachapelle, ${ }^{13}$ Maureen A. McCall, ${ }^{15}$ Robert K. Koenekoop, ${ }^{13}$ Arthur A.B. Bergen, ${ }^{5}$ Maarten Kamermans, ${ }^{7,16, *}$ and Ronald G. Gregg ${ }^{4,15, *}$

${ }^{1}$ Cole Eye Institute, Cleveland Clinic, Cleveland OH 44195, USA; ${ }^{2}$ Cleveland Veterans Affairs Medical Center, Cleveland, $\mathrm{OH} 44106$, USA; ${ }^{3}$ Cleveland Clinic Lerner College of Medicine of Case Western Reserve University, Cleveland, OH 44195, USA; ${ }^{4}$ Department of Biochemistry, University of Louisville, Louisville, KY 40202, USA; ${ }^{5}$ Department of Clinical and Molecular Ophthalmogenetics, Netherlands Institute for Neuroscience, The Royal Netherlands Academy of Arts and Sciences, Amsterdam, The Netherlands; ${ }^{6}$ The Jackson Laboratory, Bar Harbor, ME 04609, USA; ${ }^{7}$ Department of Retinal Signal Processing, Netherlands Institute for Neuroscience, The Royal Netherlands Academy of Arts and Sciences, Amsterdam 1105 BA, The Netherlands; ${ }^{8}$ Neuroscience Institute, Department of Pharmacology and Toxicology, Morehouse School of Medicine, Atlanta, 
GA 30310, USA; 'Department of Ophthalmology, Emory University, Atlanta, GA 30322 , USA; ${ }^{10}$ Department of Ophthalmology, Erasmus University Medical Center, Rotterdam 3015 CE, The Netherlands; ${ }^{11}$ Bartime'us Institute for the Visually Impaired, 3702 AD Zeist, The Netherlands; ${ }^{12}$ Retina Foundation of the Southwest, Dallas, TX 75231, USA; ${ }^{13}$ Paediatric Ophthalmology Division, Montreal Children's Hospital, McGill University Health Centre, Montreal H3H 1P3, Quebec, Canada; ${ }^{14}$ Eye Research Institute, Oakland University, Rochester, MI 48309, USA; ${ }^{15}$ Department of Ophthalmology and Visual Sciences, University of Louisville, Louisville, KY 40202, USA; ${ }^{16}$ Department of Neurogenetics, Academic Medical Center, Amsterdam 1105 AZ, The Netherlands

*Correspondence: m.kamermans@nin.knaw.nl (M.K.), ron.gregg@louisville.edu (R.G.G.) DOI 10.1016/j.ajhg.2011.12.006. (C) 2012 by The American Society of Human Genetics. All rights reserved.

Complete congenital stationary night blindness (cCSNB) is a clinically and genetically heterogeneous group of retinal disorders characterized by nonprogressive impairment of night vision, absence of the electroretinogram (ERG) b-wave, and variable degrees of involvement of other visual functions. We report here that mutations in GPR179, encoding an orphan G protein receptor, underlie a form of autosomalrecessive cCSNB. The Gpr179 nob5/nob5 mouse model was initially discovered by the absence of the ERG b-wave, a component that reflects depolarizing bipolar cell (DBC) function. We performed genetic mapping, followed by next-generation sequencing of the critical region and detected a large transposon-like DNA insertion in Gpr179. The involvement of GPR179 in DBC function was confirmed in zebrafish and humans. Functional knockdown of GPR179 in zebrafish led to a marked reduction in the amplitude of the ERG b-wave. Candidate gene analysis of GPR179 in DNA extracted from patients with cCSNB identified GPR179-inactivating mutations in two patients. We developed an antibody against mouse GPR179, which robustly labeled DBC dendritic 
terminals in wild-type mice. This labeling colocalized with the expression of GRM6 and was absent in Gpr179nob5/nob5 mutant mice. Our results demonstrate that GPR179 plays a critical role in $\mathrm{DBC}$ signal transduction and expands our understanding of the mechanisms that mediate normal rod vision.

Congenital stationary night blindness (CSNB) is a severe disability that impairs night vision. Complete CSNB (cCSNB) is a genetically heterogeneous form of the disorder that is caused by mutations in genes that are required for signal transduction through retinal depolarizing bipolar cells (DBCs). ${ }^{1-8}$ The function of photoreceptors and DBCs can be assessed noninvasively with the electroretinogram (ERG), and their lightinduced activities are reflected in the a-wave and b-wave, respectively. ${ }^{9}$ Individuals with cCSNB and animal models of the disorder have an ERG waveform that lacks the b-wave because of a failure to transmit the photoreceptor signal through the DBCs. Depolarization of the DBCs is initiated by a metabotropic glutamate receptor-mediated $(\mathrm{GRM} 6)^{10}$ modulation of a transient receptor potential melastatin 1 cation channel (TRPM1). ${ }^{11-13}$ This $\mathrm{G}$ protein signal transduction cascade utilizes $\mathrm{Gao}, 1^{14} \mathrm{G} \beta^{5,15}$ and depends on the auxiliary protein nyctalopin. ${ }^{16,17}$ Mutations GRM6 (MIM604096), TRPM1 (MIM 613216), or NYX (MIM 300278), which encodes nyctalopin, all can cause cCSNB in humans. ${ }^{1-8}$ Mice with mutations in Grm6, Trpm1, Gna0, and Gnb5 or Nyx also have a no b-wave (nob) ERG phenotype. ${ }^{10-16,18-20}$ In this report, we define a critical role for GPR179, a previously uncharacterized orphan $G$ protein receptor, in the DBC signal transduction cascade and in human cCSNB. Specifically, mutations in GPR179 in humans are responsible for a form of cCSNB. Consistent with this result, nob5 mice have a mutation in $\operatorname{Gpr179}\left(G p r 179^{\text {nob5/nob5}}\right)$ and a nob ERG phenotype. Finally, zebrafish, whose Gpr179 expression is knocked down via morpholino injection, have a reduced ERG b-wave amplitude. 
The nob5 mouse arose as a spontaneous mutation in a colony of $\mathrm{C} 3 \mathrm{H}$ mice and was identified via ERG when this line was crossed to a line of $\mathrm{C} 3 \mathrm{H}$ mice lacking the rd1 mutation $(\mathrm{C} 3 \mathrm{H}-\mathrm{fp} / \mathrm{p})$. To identify the causative mutation, we crossed affected nob5 mice to wild-type (WT) C57BL/6J mice and the resulting F1 mice were intercrossed to generate a segregating mapping cross. We identified F2 progeny homozygous for the nob5 locus by ERG and used them to map the phenotype by using a genome-wide screen with 103 simple sequence length polymorphic markers distributed throughout the genome. ${ }^{21}$ Initial mapping localized the gene to chromosome 11 . Subsequently, > 600 additional informative meiosis refined the map location of the nob5 locus to between D11Mit54 and D11Mit67. This $1.3 \mathrm{Mb}$ region contains over 90 genes, none of which were known to be involved in $\mathrm{DBC}$ signal transduction or had been identified in molecular analyses of enriched pools of DBCs. ${ }^{22-24}$ To identify the mutation underlying the nob5 phenotype we used genome capture and high-throughput sequencing. Comparison of the sequence encompassing the critical region in nob5/nob5 and WT $\mathrm{C} 3 \mathrm{H}$ mice revealed the presence of an insertion in intron 1 of Gpr179 (Figure 1A). The next-generation sequence data provided only $10 \mathrm{bp}$ of sequence on either side of the insertion, but these data suggested the insertion wasa transposable element. To examine this directly, we used PCR to amplify the insertion and its flanking intronic DNA. This revealed the presence of the predicted $1.3 \mathrm{~kb}$ fragment in WT mice and a $7.8 \mathrm{~kb}$ fragment in homozygous affected littermates, indicating the insertion is $\sim 6.5 \mathrm{~kb}$ (Figure 1A). Both bands were seen in heterozygotes. Henceforth, the mutant nob5 allele will be referred to as Gpr179 nob5. Sequence analyses of the ends of the insertion indicated it was an endogenous retroviral element of the ERV2 class (Figure 1B). To evaluate the impact of this insertion on Gpr179 expression, we used a quantitative intronspanning Taqman RT-PCR assay to determine Gpr179 mRNA levelsof WT and Gpr179nob5/nob5 retinas (Figure 1C). The expression of mRNA representing Gpr179 in the Gpr179 nob5/nob5 
retina was decreased more than 800 -fold compared to the expression of mRNA in the WT retina. These data indicate that the $G p r 179^{\text {nob5/nob5 }}$ phenotype is caused by a large insertion mutation in intron 1 of Gpr179; this insertion dramatically reduces gene expression and probably represents a null allele. 


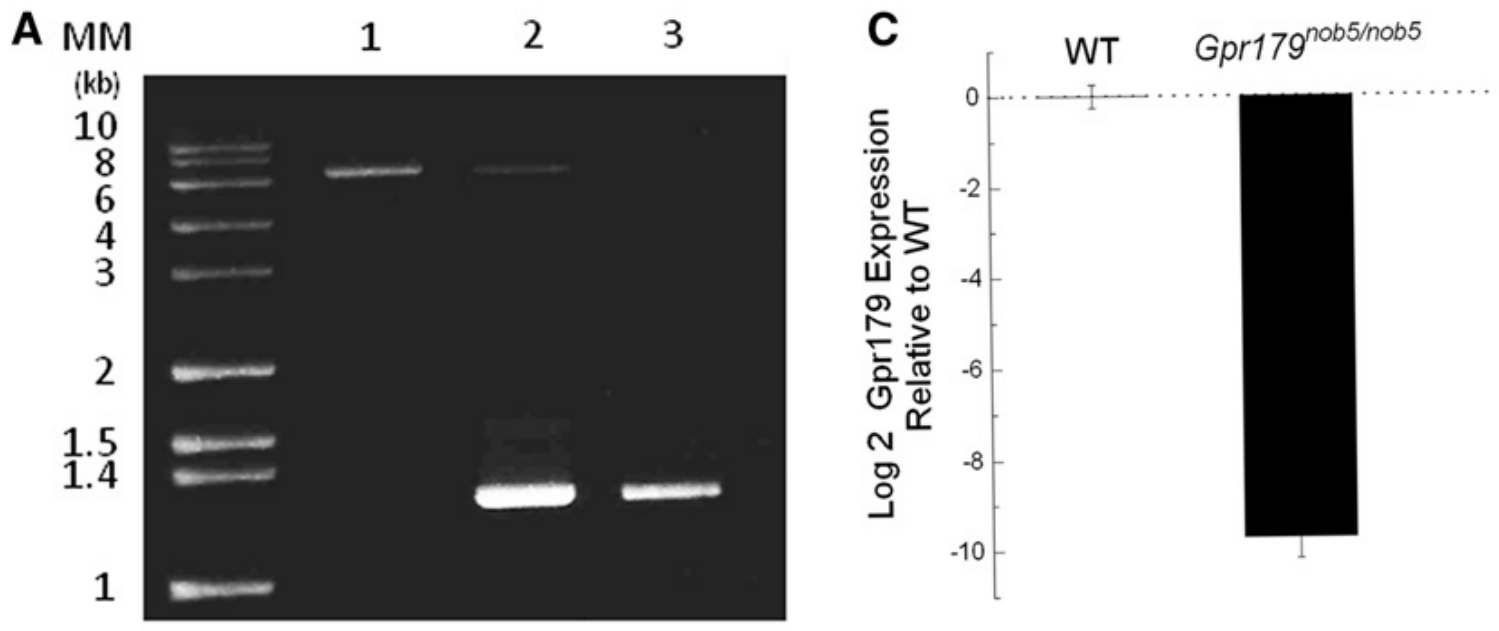

B

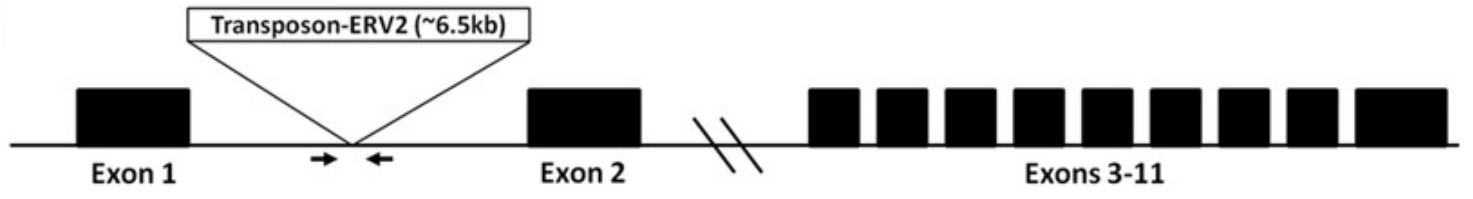

Figure 2: Transposable Element Disrupts Gpr179 Expression in nob5 Mouse (A) PCR fragments from Gpr179 $9^{\text {nob5/nob5 }}$ (lane 1), Gpr179 ${ }^{\text {nob5/4 }}$. (lane 2), and WT C3H (lane 3). The insertion is $\sim 6.5 \mathrm{~kb}$. (B) Schematic exon map of Gpr179 indicating location of nob5 insertion mutation. The arrows indicate location of PCR primers used in (A). (C) Quantitative PCR of Gpr179 cDNA generated from mRNA isolated from retinas of WT and Gpr179 ${ }^{\text {nob5/nob5 }}$ mice. Expression of Gpr179 was normalized to that of 18S RNA and is relative to the Gpr179 expression in WT. The error bars indicate mean \pm standard deviation for three mice. Gpr179 expression in Gpr179 ${ }^{n o b 5 / n o b 5}$ retina is significantly reduced $(p<0.0005)$. All animal studies were approved by the local institutional animal care and use committees and conformed to all regulatory standards. 
A series of dark-adapted ERGs obtained from representative WT, Gpr179nob5/p, and Gpr179 nob5/nob5 mice are shown in (Figure 2A). Throughout the stimulus range examined, WT ERGs are dominated by a positive polarity b-wave, which increases in amplitude with increasing flash luminance and reflects the light-induced activity of DBCs. ${ }^{25}$ At higher flash luminance, the b-wave was preceded by a negative polarity awave, reflecting the light-induced closure of cation channels along rod photoreceptor outer segments. ${ }^{26}$ ERG responses in heterozygous Gpr179 ${ }^{\text {nob5/+ }}$ mice resembled the responses of WT mice, consistent with autosomal-recessive inheritance. In contrast, whereas large a-waves are obtained from homozygous Gpr179 nob5/nob5 mice, these responses lack the b-wave component, revealing slow PIII, an ERG component generated by the radial Müller glial cells. ${ }^{27}$ Summary plots for the major components of the dark-adapted ERGs are shown in Figure 2B. ERG a-wave amplitudes were comparable across the three genotypes and the b-waves of Gpr179 nob5/+ and WT mice were indistinguishable. The b-wave component is absent in Gpr179 nob5/nob5 mice, and therefore, these data are not plotted. This ERG phenotype, in which the b-wave is absent while the a-wave is preserved, indicates that rod phototransduction is unaffected in Gpr179 nob5/nob5 mice, whereas synaptic transmission between photoreceptors and DBCs, or DBC activity itself, is grossly abnormal. ${ }^{28}$ Light-adapted ERGs obtained from representative WT, Gpr179 ${ }^{\text {nob5/+ }}$, and Gpr179 $9^{\text {nob5/nob5 }}$ mice are shown in Figure 2C. In WT mice, the cone ERG was dominated by the positive polarity b-wave and higher frequency oscillatory potentials, which reflect activity through the DBC pathway. ${ }^{29}$ In contrast, cone ERGs of Gpr179 nob5/nob5 mice are electronegative. Summary plots for cone ERGs recorded from all three genotypes are shown in Figure 2D. When cone ERG amplitude is measured from the negative trough to the following positive peak, the Gpr179 nob5/nob5 response is reduced in amplitude, whereas those from WT and Gpr179 1 nob5/+ heterozygotes are comparable. The Gpr179 nob5/nob5 ERG phenotype is 
essentially indistinguishable from those of mouse mutants for other proteins involved DBC signal transduction, GRM6, ${ }^{10,19,20}$ TRPM1, ${ }^{11-13}$ and $\mathrm{NYX},{ }^{16,18}$ protein required for correct localization of TRPM1 channels in DBC dendrites. ${ }^{17}$ 
A

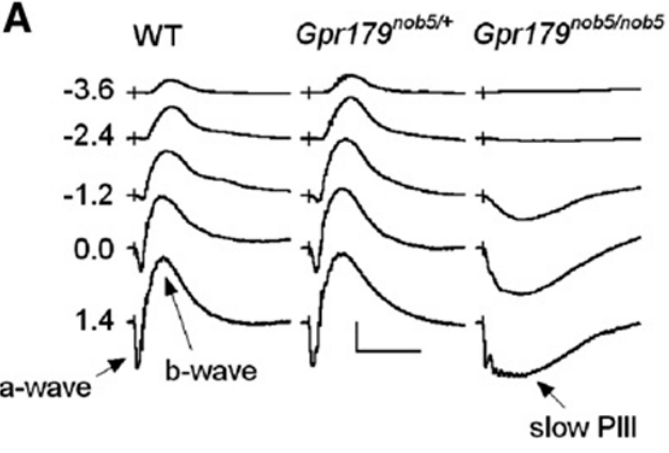

C

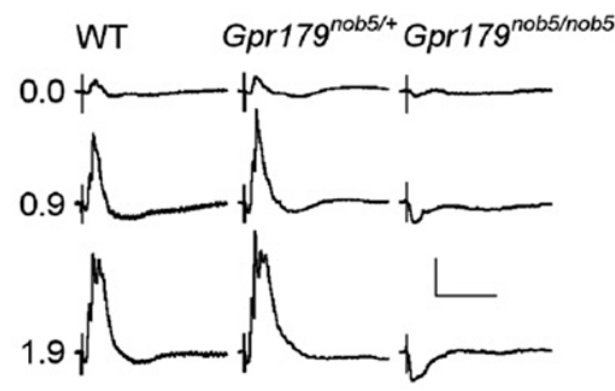

B

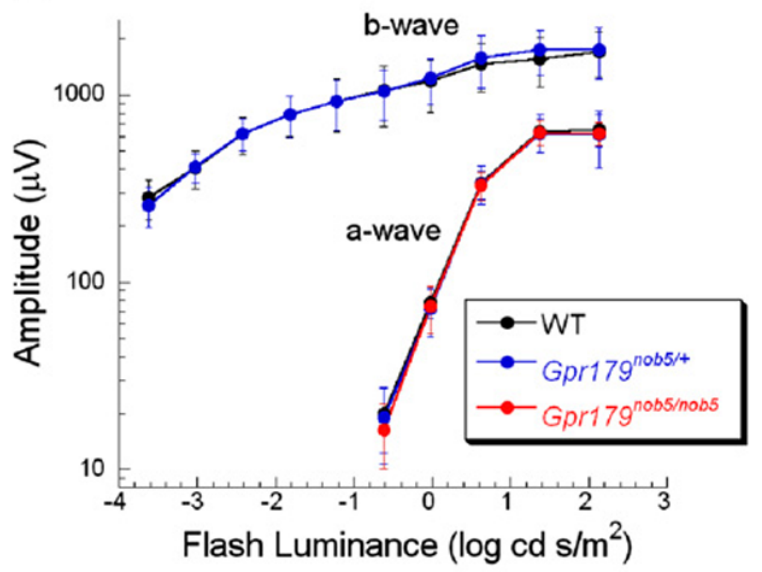

D

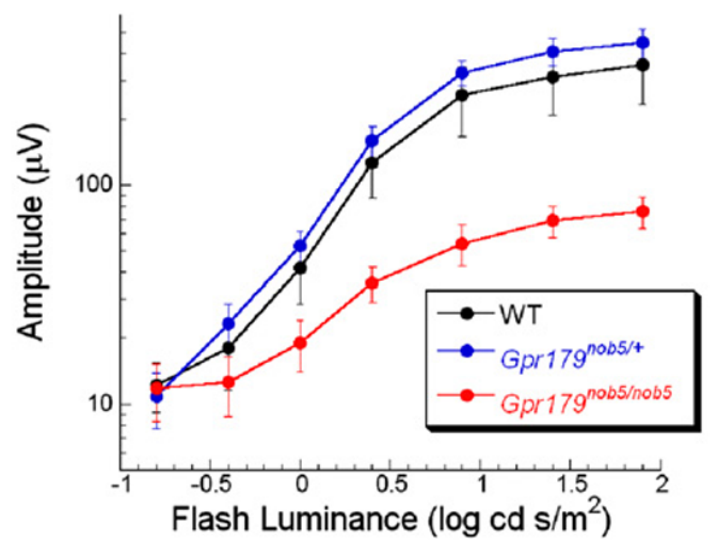

Figure 3: ERG Phenotype of Gpr179 ${ }^{\text {nob5/nob5 }}$ Mice ERGs were recorded from mice anesthetized with ketamine $(80 \mathrm{mg} / \mathrm{kg})$ and xylazine $(16 \mathrm{mg} / \mathrm{kg})$ after overnight dark adaptation via a published procedure. ${ }^{16}$ (A) Dark-adapted ERG series obtained from representative WT (left), Gpr179 ${ }^{\text {nob5/+ }}$ (middle), and Gpr179 ${ }^{\text {nob5/nob5 }}$ (right) littermates at 6 months of age. The scale bars indicates $100 \mathrm{~ms}$ and $500 \mathrm{mV}$. Values to the left of each row of waveforms indicate flash luminance in log cd s/m2. (B) Luminance- response functions for the major components of the darkadapted ERG. The b-wave component is absent in Gpr179 therefore these data are not plotted. (C) Cone-mediated ERG series obtained from WT (left), Gpr179 ${ }^{\text {nob5/+ }}$ (middle), and Gpr179 nob5/nob5 (right) littermates at 6 months of age. Scale bar indicates $100 \mathrm{~ms}$ and $100 \mathrm{mV}$. (D) Luminance- response functions for the cone ERG b-wave. Values to the left of each row of waveforms indicate flash luminance in $\log \mathrm{cd} \mathrm{s} / \mathrm{m} 2$. 
GPR179 encodes a predicted orphan G protein-linked receptor that has not been previously characterized in any cell or tissue. The nob5 phenotype predicts that the GPR179 gene product is required for DBC function and therefore should be present in DBCs. Because DBCs receive input from photoreceptors via ribbon synapses in the outer plexiform layer (OPL), we initially characterized gross retinal morphology and OPL ultrastructure of the Gpr179 nob5/nob5 retina. However, all cellular and synaptic layers appeared normal in the Gpr179 ${ }^{\text {nob5/nob5 }}$ retina (Figure 3A). Furthermore, Gpr179 nob5/nob5 ribbon synapses are indistinguishable from WT (Figure 3B). A normal retinal morphology is typical of all other mouse models of cCSNB. ${ }^{10,12,13,18,30}$

To examine the cellular localization of GPR179 in the mouse retina, we developed a polyclonal sheep antibody to a peptide (KVQEETPGEDLDRPVLQKR) located within the amino terminal extracellular domain. Retinal cryosections from Gpr179 $9^{\text {nob5/+ }}$ and Gpr179 ${ }^{\text {nob5/nob5 }}$ littermates were reacted with our antibody to GPR179 and an antibody against PKCa to label rod DBCs. In Gpr179 ${ }^{\text {nob5/+ }}$ retina, PKCa labeled the entire rod DBC (Figures 3C and 3E), whereas the GPR179 antibody produced a punctuate-labeling pattern (Figures 3D and 3E), which corresponds to the location of the OPL. In the Gpr179 ${ }^{\text {nob5/nob5 }}$ retina, labeling for PKCa (Figures $3 \mathrm{~F}$ and $3 \mathrm{H}$ ) is comparable to Gpr179 $9^{\text {nob5/nob5 }}$ and labeling for GPR179 is absent (Figures $3 G$ and $3 \mathrm{H}$ ), consistent with decreased Gpr179 mRNA expression in Gpr179 ${ }^{\text {nob5/nob5 }}$ retinas (Figure 1C). The punctuate-labeling pattern of GPR179 in the OPL is typical of proteins that are localized to the dendritic tips of DBCs, including GRM6, TRPM1 and NYX.12,16 To confirm this localization, we double-labeled retinal sections with antibodies to GPR179 (Figure 3I) and GRM6 (Figure 3J) The punctate labeling for GPR179 colocalizes with GRM6 (Figure $3 \mathrm{~L})$, the glutamate receptor known to mediate signaling in DBCs. These data show that GPR179 is expressed on the dendritic terminals of DBCs. GPR179 is not expressed elsewhere in the retina (data not shown). The combined ERG, genetic and 
immunohistochemical data argue strongly that the Gpr179 nob5/nob5 mouse phenotype is caused by the insertion and results in a functionally null allele of GPR179. 

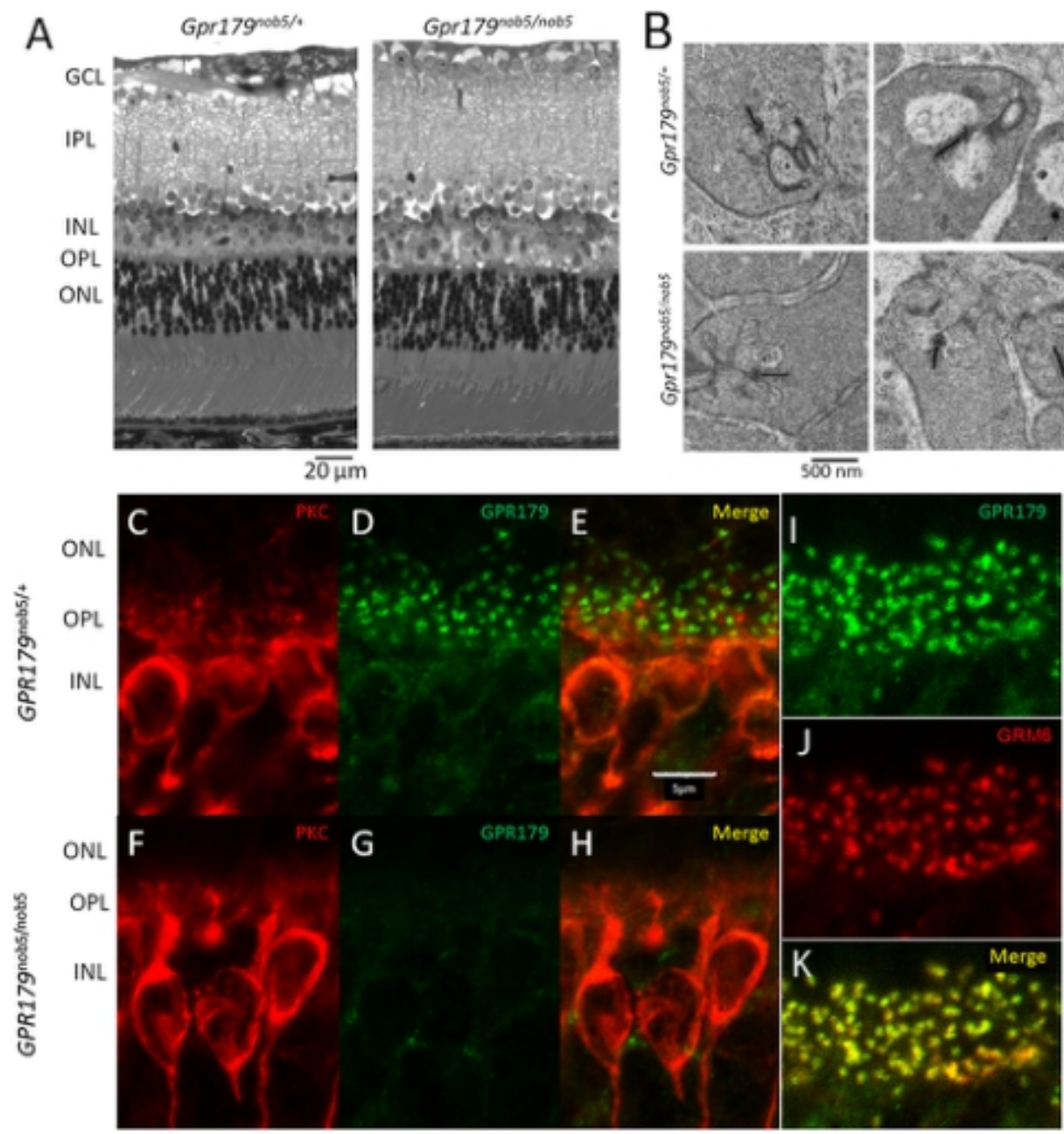

Figure 4: Anatomical Phenotype of Gpr179 ${ }^{\text {nob5/nob5 }}$ Retina (A) Retinal crosssections obtained from Gpr179 ${ }^{n o b 5 /+}$ and Gpr179 ${ }^{\text {nob5/nob5 }}$ mouse retinas fixed with $2.5 \%$ glutaraldehyde $/ 2 \%$ paraformaldehyde and prepared according to published procedures. ${ }^{35}$ (B) Electron micrographs of ribbon structures in Gpr179 $9^{\text {nob5/+ }}$ and Gpr179 ${ }^{\text {nob5/nob5 }}$ mouse retinas prepared according to published procedures. (C-K) Confocal immunohistochemistry of retinas fixed with $4 \%$ paraformaldehyde for $15 \mathrm{~min}$ and prepared according to published procedures. ${ }^{16}$ The scale bar indicates $5 \mu \mathrm{m}$. (C-E) Gpr179 ${ }^{\text {nob5/+ }}$ retina labeled with antibodies against (C) PKCa, (D) GPR179, and (E) merge of (C) and (D). (F-H) Gpr179 ${ }^{\text {nob5/nob5 }}$ retina labeled with antibodies against (F) PKCa, (G) GPR179, and (H) merge of (F) and (G). (I-K) Gpr179 ${ }^{\text {nob5/+ }}$ retina labeled with antibodies for (I) GPR179, (J) GRM6, and (K) merge of I-J. The following antibodies were used: GPR179; affinity purified polyclonal sheep anti-GPR179 peptide (KVQEETPGEDLDRPVLQKR), 1:1,000; mouse monoclonal anti-ctbp2/Ribeye, 1:1,000 (BD Biosciences); guinea pig antiGRM6, 1:1000 (see Koike et al.12); rabbit anti PKCa (1:1,000, Sigma). The following secondary antibodies were used: Alexa-488 donkey anti-sheep, Alexa-555 goat anti-rabbit, and Alexa-633 goat anti-guinea pig (all at 1:1,000; Invitrogen). 
To directly determine whether reduced GPR179 expression could recapitulate the reduced b-wave phenotype, we used morpholino knockdown in zebrafish, which have a single copy of gpr179 in their genome. We injected 1 cell stage zebrafish embryos with morpholinos (MOs) targeted against the Gpr179 translation start site (MOGpr179 50-GCCCATACTTTTAGCAACTGCTTCT-30), and recorded ERGs at 4-6 days post fertilization. As comparisons, MOs against either the nyx translation start site (MONyx 50-GAtGAAACACATCACTGGCTTC-30) ${ }^{31}$ or control (C-MO 50ССTСTTACCTCAGTTACAATTTATA-30) were injected. Embryos injected with MO Gpr179 had a significantly reduced ERG b-wave amplitude, similar to embryos injected with MO-Nyx (positive control) (Figures 4A and 4B). The b-wave was unaffected following injection of the control MO (Figure 4B). The b-wave/a-wave ratio decreased from $3.79 \pm 0.46(n=4)$ in control to $0.75 \pm 0.11(n=7)$ in Gpr179-MO $(p=0.005)$.

These results indicate that gpr179 expression is required for normal DBC function in zebrafish. 
A

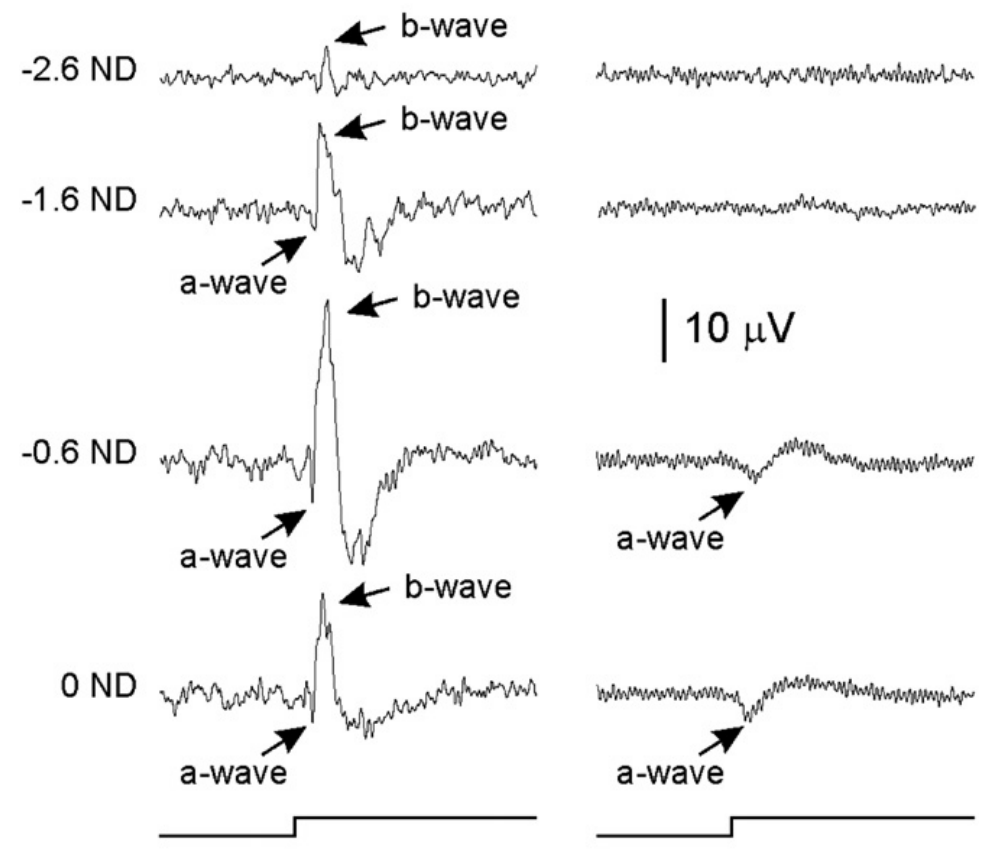

B

Control MO-gpr179 MO-NYX

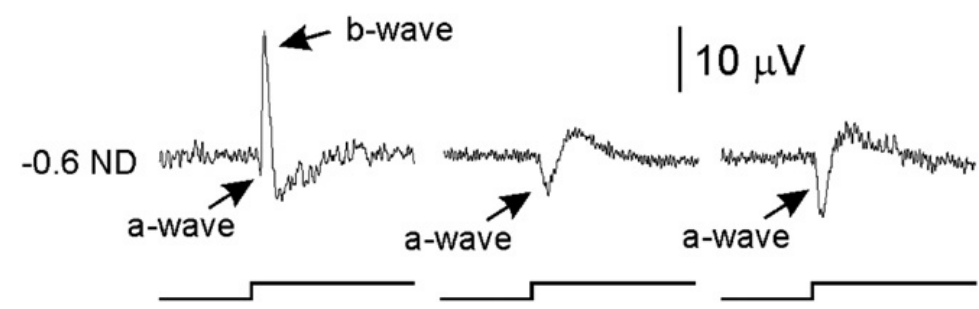

Figure 4: GPR179 Knockdown in Zebrafish Decreases ERG b-Wave Injected MOs (Gene Tools) were designed against the gpr179 translation site (MO 50GCCCATACTTTTAGCAACTGCTTCT-30), which occurs as a single copy in the zebrafish genome, or the nyx translation site (MO 50GATGAAACACATCACTGGCTTC-30) or were a standard control (MO 50CСTCTTACCTCAGTTACAATTTATA-30). In each case, $30 \mathrm{ng}$ of $\mathrm{MO}$ was injected into the chorions of onecell- stage zebrafish embryos. ERGs were recorded from larvae at 4-6 days post fertilization with a $1 \mathrm{~s}$ stimulus after $30 \mathrm{~min}$ of dark adaption, as previously described. ${ }^{36}$ (A) ERGs from embryos injected with control (left column) or MOgpr179 (right column) MOs and tested at four flash intensities. Flash intensity at $0 \mathrm{log}$ is $9.3 \mathrm{~W} / \mathrm{m} 2$. Neutral density (ND) filters reduced the intensity by the indicated amount in log units. The b-wave amplitudes obtained from MO-gpr179 injected embryos were strongly reduced at all intensities. The $b$ wave/a-wave ratio decreased from $3.79 \pm 0.46(n=4)$ in control to $0.75 \pm 0.11(n=$ 7 ) in MO-gpr179 $(p=0.005)$, showing that a specific knockdown of gpr179 reduces DBC function. (B) The magnitude of b-wave reduction for MO-gpr179 and MO-nyx were comparable. Injections of MO-control did not affect the ERG (data not shown). 
Mutations in NYX, GRM6 or TRPM1 have been identified in patients with cCSNB. ${ }^{1-8}$ However, a small number of individuals with cCSNB did not bear mutations in any of these genes.8 To evaluate the potential involvement of GPR179 in cCSNB patients lacking DBC function, we sequenced the 11 exons and flanking splice sites of the human gene in 44 patients (see Table 1 for primers). All human studies were undertaken with the approval of the appropriate institutional review board. We identified two probands with inactivating mutations in the GPR179 gene. Proband 1 had no family history of night blindness or consanguinity and was 10 years old at the time of diagnosis. He presented with $20 / 70$ best corrected visual acuities, mild myopic refractive error, congenital nystagmus, a history of early onset nightblindness, a normal retinal appearance and full Goldmann visual fields. ERGs obtained under ISCEV standard conditions from proband 1 are shown in Figure 5A. Under darkadapted conditions (upper traces), the ERG b-wave recorded to a low luminance stimulus was markedly reduced in amplitude, whereas the ERG obtained to a high flash luminance had a robust a-wave without the subsequent b-wave seen in controls (middle traces). Under light-adapted conditions (lower pair of traces), the ERG waveform showed a square a-wave but retained a late positive ERG component. ERGs of proband 2 showed a similar selective absence of the dark-adapted b-wave and a square light-adapted ERG a-wave (data not shown). These ERG abnormalities have been uniquely associated with human cases of DBC dysfunction32-34 and readily cCSNB from other retinal disorders with a reduced bwave, such as incomplete CSNB (MIM 300071 and 610427). ${ }^{33}$

Proband 1 was a compound heterozygote for two frameshift mutations in GPR179, c.187delC and c.984delC (NM_001004334.2) resulting in predicted protein truncations p.Leu63Serfs ${ }^{*} 12$ and p.Ser329Leufs* 4 , respectively (Figure 5B). The premature chain termination is expected to result in functional null alleles. The probands' unaffected parents were each heterozygous for one of the mutations (Figure 5B). 
Proband 2 was 20 years old at the time of diagnosis. She is of Norwegian descent and presented with rotatory nystagmus, a very unusual blond fundus, and congenital nightblindness and was able to see $20 / 30$ with a -12.00 D prescription. Although not known to be related, proband 2 also carried the c.984delC frameshift mutation identified in proband 1 , suggesting that this might be a founder mutation. Proband 2 carried a second mutation, c.659A $>\mathrm{G}$, that would result in missense mutation, p.Tyr220Cys. (Figure 5C). The three variants in probands 1 and 2 were not present in 210 healthy control chromosomes. Two out of three Alamut analyses, which predict mutation impact on function, classified the p.Tyr220Cys as potentially pathogenic. Moreover, by introducing a new cysteine into the GRP179 protein, the mutation is likely to impact its structure. The functional importance of Tyr220 is supported by its conservation across species ranging from human to Tetraodon (Figure 5D). DNA of family members of proband 2 was not available for segregation analysis. 
A

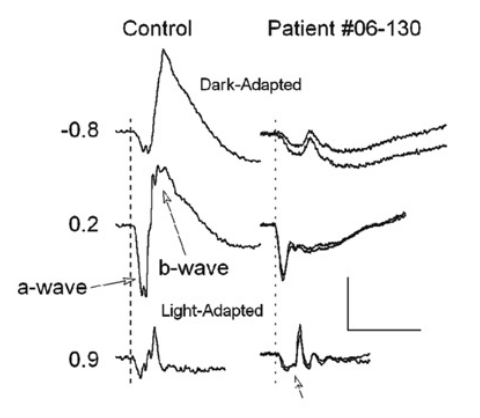

B
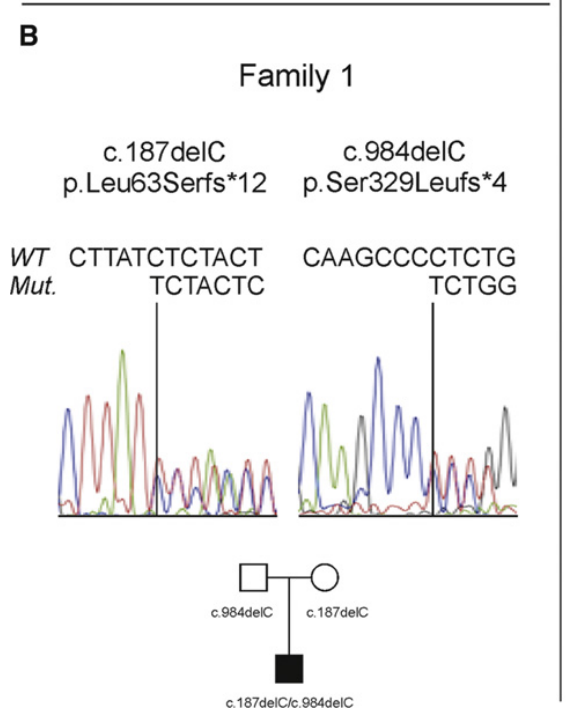

C

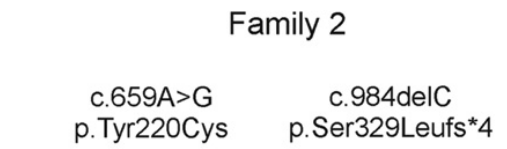

WT ATG GATATG TGG CAAGCCCCTCTG Mut. G TCTGG

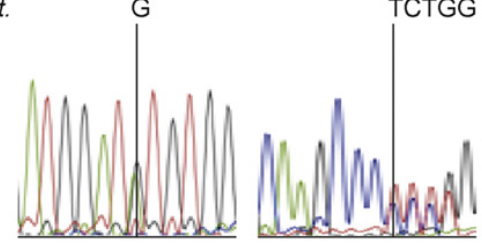

D
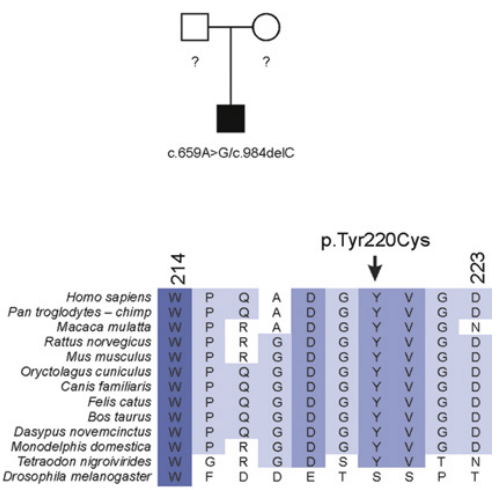

Figure 5: GPR179 Mutations Are Present in Two Probands with AutosomalRecessive cCSNB (A) ERGs obtained from a control subject and proband 1 for a standard series of stimulus conditions that allow rod- and cone-mediated responses to be evaluated. For proband 1 (patient 06-130), the two records indicate ERGs obtained from the two eyes. Under dark-adapted conditions, ERGs obtained from the proband had markedly reduced b-waves. Under light-adapted conditions (30 cd/m2), the cone ERG had a squared a-wave (arrow). Values indicate flash luminance in log cd s/m2. (B and C) GPR179 (accession number NM_001004334.2) exons were sequenced from DNA samples isolated from 44 patients with cCSNB. Chromatograms containing the mutant sequences found in probands 1 (B) and 2 (C). In addition to the chromatogram, each subsection shows the mutation, the predicted impact on the amino acid sequence, and the segregation pattern. The pedigree for proband 1 shows that he inherited one mutant GPR179 allele from each parent (c.187delC and c.984delC), who had normal vision (data not shown). The parents of proband 2 were not available for analyses. (D) Comparison of the region of GPR179 containing the amino acid substitution (p.Tyr220Cys) identified in proband 2 across phyla. With the exception of Drosophila melanogaster, Tyr220 is conserved for every species for which data were available. In general, this region of the protein is highly conserved (the shade of blue indicates the amount of conservation; dark blue indicates the most conserved). The study followed the tenets of the Declaration of Helsinki and was approved by the ethics committee of the Academic Medical Centre, Amsterdam. All participants provided signed informed consent for participation in the study. 
The combined data indicate that GPR179 is required for DBC signal transduction and that mutations that disrupt the function of GPR179 cause a recessive form of cCSNB. Although Gpr179nob5/nob5 mice lack expression of GPR179, we have not detected any anatomical defect in the retina, and the mice have no apparent health problem. Similarly, the two human patients we have identified with cCSNB and mutations in GPR179 have no other known health problems. Given the ERG phenotype in mice, zebrafish, and humans, as well as the colocalization of GPR179 with GRM6, we postulate that GPR179 plays a critical role in DBC signal transduction, possibly by forming heterodimers with GRM6. Future studies will be required to define the specific role of GPR179 in this process. The availability of the Gpr179 ${ }^{\text {nob5/nob5 }}$ mouse model will be an important tool with which to address this question. 


\begin{tabular}{|c|c|c|c|}
\hline Name & Forward Primer $\left(5^{\prime} \rightarrow 3^{\prime}\right)$ & Reverse Primer $\left(5^{\prime} \rightarrow 3^{\prime}\right)$ & Amplicon Size (bp) \\
\hline GPR179-EXON1_1 & GACTGCAGCCAGCCTCTG & GCATGTTGAGAAAATTGGCG & 402 \\
\hline GPR179-EXON1_2 & AGATGCCCAGCAGCTATCAC & TCAACACTCGCTTCTTCAGG & 410 \\
\hline GPR179-EXON1_3 & ACCTTTAACCCTCCACCAGG & GGACCTGTACTTCCTTCCCC & 410 \\
\hline GPR179-EXON2 & AAAGTGCCATAATTCCGAGC & GAGATGCTGGGGTAGCTTTG & 330 \\
\hline GPR179-EXON3 & GACCCAAGTCCCATGGATAG & GTACCCTCCCAACCATCCTC & 261 \\
\hline GPR179-EXON4 & GGCTGTGGTGACTATCAGGG & TAGTCTCAGGCCTCCAGTTG & 386 \\
\hline GPR179-EXON5 & ACAGGGTGAAGCCTTAGCTC & GTGCCAGGTTTTGGCTATG & 241 \\
\hline GPR179-EXON6 & TGGTGATGGCTCTATTGCAG & ATGAGTGAGCTGGGGTGG & 284 \\
\hline GPR179-EXON7 & CAGCAATGGACAGAGACCAG & CCAGAACAAGAGGAACCCTG & 401 \\
\hline GPR179-EXON8 & TCTTTCAGAGAAAGGGGTGG & GTAGCAGTGTTGCCAGGATG & 313 \\
\hline GPR179-EXON9 & CTAAGCGTATCAGGGTTGGG & CTCCACAGTGGCCCTGAC & 274 \\
\hline GPR179-EXON10 & TGAGTGCAGGAGTGGACAAG & GACTTGCAGTGGCACATAGC & 311 \\
\hline GPR179-EXON11-1 & AAGGGTGGAGGAACAGACAG & CGCTGGCTGACCTGAAG & 497 \\
\hline GPR179-EXON11-2 & GACCCGCCTCTTCTTGACTC & TAGAAACCTGGTGCCTGATG & 472 \\
\hline GPR179-EXON11-3 & AGGCCATCAGCCAGGAG & GCCCTGCTTTTCTCTACTGC & 473 \\
\hline GPR 179-EXON11-4 & CAGCCAAGCAAGAAAATGTG & TCTGTTGGTTCTGGAGGGAC & 471 \\
\hline GPR 179-EXON11-5 & CGTTCTCGGAGCACCTACAG & GCTGACGCGTTTCTGATTC & 481 \\
\hline GPR 179-EXON11-6 & ATCAAAAGAAACCCCTGTCG & TCAGGGGTATGAGCTTCTGG & 480 \\
\hline GPR 179-EXON11-7 & CTGGGAGACGAGTGAAGGAG & AAGTCTCCTCCCGGTTTCTG & 474 \\
\hline GPR179-EXON11-8 & AGTGAAGGAGGCGAGGATG & TCTCCCTGGGACAAACTGAC & 463 \\
\hline GPR 179-EXON11-9 & AATGCTGAGTCTGGGGACAG & GTCTTGAGGACGTGGTTGTG & 529 \\
\hline GPR 179-EXON11-10 & TGGAAAATCGGAAATCGAAG & ACCTGGATGCTGAGAACAGG & 460 \\
\hline GPR 179-EXON11-11 & CCCAGGGAGAGAGCTGTTAC & GCTGCTGGGCAAGTCTGAG & 474 \\
\hline GPR 179-EXON11-12 & GCCTGTTCTCAGCATCCAG & TGGGTTATGTGTTCTGGGAAG & 482 \\
\hline GPR 179-EXON11-13 & GAGTAGTGAAGTGGCAGAGGG & CTGAACTGGCACACCTTTTG & 487 \\
\hline GPR 179-EXON11-14 & AATCTGACAGCAGTGCCAAG & AGGAACATCTCTCCTTGCCC & 445 \\
\hline GPR179-EXON11-15 & CTGTGGGAAGTGGTAGAGGC & TTTTCAGGAGCTGTGGGG & 473 \\
\hline GPR179-EXON11-16 & AGGGTACCATGGCAGACATC & TTCAGCTCTTTGGGAACACC & 486 \\
\hline
\end{tabular}




\section{Acknowledgments}

We are grateful to Mathieu Gauvin for assistance with data analyses, Wim de Graaff for the morpholino injections in zebrafish larvae, and Takahisa Furakawa for the gift of the GRM6 antibody. We also thank the patients for their participation. This research was supported by grants from the National Institutes of Health (R21EY021852 to N.S.P. and R.G.G.; R01EY12354 to R.G.G.; R01EY014701 to M.A.M.; RR017890 to A.F.X.G.; R01EY016501 and P30CA34196 to P.M.N.; R01EY004864 and P30EY006360 toP.M.I.; and R01NS43459 and R01EY020821 to G.T.); the Veterans Administration Medical Research Service; the Netherlands Organization for Scientific Research (ZonMW NWO to M.K.); ODAS and European Commission FP7 Grant RETICIRC HEALTHF2-2009- 223156 (to M.K.); Algemene Nederlandse Vereniging ter Voorkoming van Blindheid (to A.A.B.B.); the Foundation Fighting Blindness Canada (to R.K.K.); the Canadian Institutes for Health Research (to R.K.K.); Reseau Vision (to R.K.K.); Fonds de recherche du Que'bec- Sante' (to R.K.K.), a Foundation Fighting Blindness Center Grant to the Cole Eye Institute, Cleveland Clinic; and unrestricted awards from Research to Prevent Blindness to the Department of Ophthalmology, Cleveland Clinic Lerner College of Medicine; to the Department of Ophthalmology, Emory University; and to the Department of Ophthalmology and Visual Sciences, University of Louisville. P.M.I. is a recipient of an RPB Senior Scientific Investigator Award.

Received: November 1, 2011

Revised: November 21, 2011

Accepted: December 8, 2011

Published online: February 9, 2012 
References

1. Bech-Hansen, N.T., Naylor, M.J., Maybaum, T.A., Sparkes, R.L., Koop, B., Birch, D.G., Bergen, A.A., Prinsen, C.F., Polomeno, R.C., Gal, A., et al. (2000). Mutations in NYX, encoding the leucine-rich proteoglycan nyctalopin, cause $X-$ linked complete congenital stationary night blindness. Nat.Genet. 26, 319-323.

2. Pusch, C.M., Zeitz, C., Brandau, O., Pesch, K., Achatz, H., Feil, S., Scharfe, C., Maurer, J., Jacobi, F.K., Pinckers, A., et al. (2000). The complete form of Xlinked congenital stationary night blindness is caused by mutations in a gene encoding a leucine-rich repeat protein. Nat. Genet. 26, 324-327.

3. Dryja, T.P., McGee, T.L., Berson, E.L., Fishman, G.A., Sandberg, M.A., Alexander, K.R., Derlacki, D.J., and Rajagopalan, A.S. (2005). Night blindness and abnormal cone electroretinogram ON responses in patients with mutations in the GRM6 gene encoding mGluR6. Proc. Natl. Acad. Sci. USA 102, 4884-4889.

4. Zeitz, C., van Genderen, M., Neidhardt, J., Luhmann, U.F., Hoeben, F., Forster, U., Wycisk, K., Ma'tya's, G., Hoyng, C.B., Riemslag, F., et al. (2005). Mutations in GRM6 cause autosomal recessive congenital stationary night blindness with a distinctive scotopic $15-\mathrm{Hz}$ flicker electroretinogram. Invest. Ophthalmol. Vis. Sci. 46, 4328-4335.

5. Audo, I., Kohl, S., Leroy, B.P., Munier, F.L., Guillonneau, X., Mohand-Sal"d, S., Bujakowska, K., Nandrot, E.F., Lorenz, B., Preising, M., et al. (2009). TRPM1 is mutated in patients with autosomal-recessive complete congenital stationary night blindness. Am. J. Hum. Genet. 85, 720-729.

6. Li, Z., Sergouniotis, P.I., Michaelides, M., Mackay, D.S.,Wright, G.A., Devery, S., Moore, A.T., Holder, G.E., Robson, A.G., and Webster, A.R. (2009). Recessive mutations of the gene TRPM1 abrogate ON bipolar cell function and cause complete congenital stationary night blindness in humans. Am. J. Hum. Genet. 85, 711-719.

7. van Genderen, M.M., Bijveld, M.M., Claassen, Y.B., Florijn, R.J., Pearring, J.N., Meire, F.M., McCall, M.A., Riemslag, F.C., Gregg, R.G., Bergen, A.A., and Kamermans, M. (2009). Mutations in TRPM1 are a common cause of complete congenital stationary night blindness. Am. J. Hum. Genet. 85, 730-736.

8. Nakamura, M., Sanuki, R., Yasuma, T.R., Onishi, A., Nishiguchi, K.M., Koike, C., Kadowaki, M., Kondo, M., Miyake, Y., and Furukawa, T. (2010). TRPM1 mutations are associated with the complete form of congenital stationary night blindness. Mol. Vis. 16, 425-437.

9. Frishman, L.J., and Wang, M.H. (2011). Electroretinogram of human, monkey and mouse. In Adler's Physiology of the Eye, 11th ed., L.A. Levin, S.F.E. Nilsson, J. Ver Hoeve, S.M. Wu, P.L. Kaufman, and A. Alm, eds. (New York: Saunders Elsevier), pp. 480-501.

10. Masu, M., Iwakabe, H., Tagawa, Y., Miyoshi, T., Yamashita, M., Fukuda, Y., Sasaki, H., Hiroi, K., Nakamura, Y., Shigemoto, R., et al. (1995). Specific deficit of the ON response in visual transmission by targeted disruption of the mGluR6 gene. Cell 80, 757-765.

11. Shen, Y., Heimel, J.A., Kamermans, M., Peachey, N.S., Gregg, R.G., and Nawy, S. (2009). A transient receptor potential-like channel mediates synaptic transmission in rod bipolar cells. J. Neurosci. 29, 6088-6093.

12. Koike, C., Obara, T., Uriu, Y., Numata, T., Sanuki, R., Miyata, K., Koyasu, T., Ueno, S., Funabiki, K., Tani, A., et al. (2010). TRPM1 is a component of the retinal ON bipolar cell transduction channel in the mGluR6 cascade. Proc. Natl. Acad. Sci. USA 107, 332-337.

13. Morgans, C.W., Zhang, J., Jeffrey, B.G., Nelson, S.M., Burke, N.S., Duvoisin, R.M., 
and Brown, R.L. (2009). TRPM1 is required for the depolarizing light response in retinal ONbipolar cells. Proc. Natl. Acad. Sci. USA 106, 19174-19178.

14. Dhingra, A., Jiang, M.,Wang, T.L., Lyubarsky, A., Savchenko, A., Bar-Yehuda,T., Sterling, P., Birnbaumer, L., andVardi, N. (2002). Light response of retinal ON bipolar cells requires a specific splice variant of Galpha(o). J. Neurosci. 22, 4878-4884.

15. Rao, A., Dallman, R., Henderson, S., and Chen, C.K. (2007). Gbeta5 is required for normal light responses and morphology of retinal ON-bipolar cells. J. Neurosci. 27, 14199-14204.

16. Gregg, R.G., Kamermans, M., Klooster, J., Lukasiewicz, P.D., Peachey, N.S., Vessey, K.A., and McCall, M.A. (2007). Nyctalopin expression in retinal bipolar cells restores visual function in a mouse model of complete $\mathrm{X}$-linked congenital stationary night blindness. J. Neurophysiol. 98, 3023-3033.

17. Pearring, J.N., Bojang, P., Jr., Shen, Y., Koike, C., Furukawa, T., Nawy, S., and Gregg, R.G. (2011). A role for nyctalopin, a small leucine-rich repeat protein, in localizing the TRP melastatin 1 channel to retinal depolarizing bipolar cell dendrites. J. Neurosci. 31, 10060-10066.

18. Pardue, M.T., McCall, M.A., LaVail, M.M., Gregg, R.G., and Peachey, N.S. (1998). A naturally occurring mouse model of X-linked congenital stationary night blindness. Invest. Ophthalmol. Vis. Sci. 39, 2443-2449.

19. Pinto, L.H., Vitaterna, M.H., Shimomura, K., Siepka, S.M., Balannik, V., McDearmon, E.L., Omura, C., Lumayag, S., Invergo, B.M., Glawe, B., et al. (2007). Generation, identification and functional characterization of the nob4 mutation of Grm6 in the mouse. Vis. Neurosci. 24, 111-123.

20. Maddox, D.M., Vessey, K.A., Yarbrough, G.L., Invergo, B.M., Cantrell, D.R., Inayat, S., Balannik, V., Hicks, W.L., Hawes, N.L., Byers, S., et al. (2008). Allelic variance between GRM6 mutants, Grm6nob3 and Grm6nob4 results in differences in retinal ganglion cell visual responses. J. Physiol. 586, 4409- 4424.

21. Taylor, B.A., Navin, A., and Phillips, S.J. (1994). PCR-amplification of simple sequence repeat variants from pooled DNA samples for rapidly mapping new mutations of the mouse. Genomics 21, 626-632.

22. Dhingra, A., Sulaiman, P., Xu, Y., Fina, M.E., Veh, R.W., and Vardi, N. (2008). Probing neurochemical structure and function of retinal ON bipolar cells with a transgenic mouse. J. Comp. Neurol. 510, 484-496.

23. Kim, D.S., Ross, S.E., Trimarchi, J.M., Aach, J., Greenberg, M.E., and Cepko, C.L. (2008). Identification of molecular markers of bipolar cells in the murine retina. J. Comp. Neurol. 507, 1795-1810.

24. Nakajima, Y., Moriyama, M., Hattori, M., Minato, N., and Nakanishi, S. (2009). Isolation of ON bipolar cell genes via hrGFP-coupled cell enrichment using the mGluR6 promoter. J. Biochem. 145, 811-818.

25. Kofuji, P., Ceelen, P., Zahs, K.R., Surbeck, L.W., Lester, H.A., and Newman, E.A. (2000). Genetic inactivation of an inwardly rectifying potassium channel (Kir4.1 subunit) in mice: Phenotypic impact in retina. J. Neurosci. 20, 5733- 5740.

26. Penn, R.D., and Hagins, W.A. (1969). Signal transmission along retinal rods and the origin of the electroretinographic a-wave. Nature 223, 201-204.

27. Samuels, I.S., Sturgill, G.M., Grossman, G.H., Rayborn, M.E., Hollyfield, J.G., and Peachey, N.S. (2010). Light-evoked responses of the retinal pigment epithelium: Changes accompanying photoreceptor loss in the mouse. J. Neurophysiol. 104, 391-402.

28. McCall, M.A., and Gregg, R.G. (2008). Comparisons of structural and functional abnormalities in mouse b-wave mutants. J. Physiol. 586, 4385-4392. 
29. Sharma, S., Ball, S.L., and Peachey, N.S. (2005). Pharmacological studies of the mouse cone electroretinogram. Vis. Neurosci. 22, 631-636.

30. Pardue, M.T., Ball, S.L., Candille, S.I., McCall, M.A., Gregg, R.G., and Peachey, N.S. (2001). nob: A mouse model of CSNB1. In New Insights into Retinal Degenerative Diseases, J.G. Hollyfield, R.E. Anderson, and M.M. LaVail, eds. (New York: Kluwer/Plenum Press), pp. 319-328.

31. Bahadori, R., Biehlmaier, O., Zeitz, C., Labhart, T., Makhankov, Y.V., Forster, U., Gesemann, M., Berger, W., and Neuhauss, S.C. (2006). Nyctalopin is essential for synaptic transmission in the cone dominated zebrafish retina. Eur. J. Neurosci. 24, 1664-1674.

32. Lachapelle, P., Little, J.M., and Polomeno, R.C. (1983). The photopic electroretinogram in congenital stationary night blindness with myopia. Invest. Ophthalmol. Vis. Sci. 24, 442-450.

33. Miyake, Y., Yagasaki, K., Horiguchi, M., Kawase, Y., and Kanda, T. (1986). Congenital stationary night blindness with negative electroretinogram. A new classification. Arch. Ophthalmol. 104, 1013-1020.

34. Alexander, K.R., Fishman, G.A., Peachey, N.S., Marchese, A.L., and Tso, M.O.M. (1992). 'On' response defect in paraneoplastic night blindness with cutaneous malignant melanoma. Invest. Ophthalmol. Vis. Sci. 33, 477-483.

35. Goldberg, A.F., Ritter, L.M., Khattree, N., Peachey, N.S., Fariss, R.N., Dang, L., Yu, M., and Bottrell, A.R. (2007). An intramembrane glutamic acid governs peripherin/rds function for photoreceptor disk morphogenesis. Invest. Ophthalmol. Vis. Sci. 48, 2975-2986.

36. Makhankov, Y.V., Rinner, O., and Neuhauss, S.C. (2004). An inexpensive device for non-invasive electroretinography in small aquatic vertebrates. J. Neurosci. Methods 135, 205-210. 


\section{APPENDIX II: GPR158/179 regulate G protein signaling by controlling localization and activity of the RGS7 complexes.}

Rockefeller University Press permits authors to reuse their own work. Orlandi et al., 2012. Originally published in The Journal of Cell Biology DOI:jcb.201202123

Cesare Orlandi ${ }^{1}$, Ekaterina Posokhova ${ }^{1}$, Ikuo Masuho ${ }^{1}$, Thomas A. Ray ${ }^{2}$; Nazarul Hasan $^{2}$, Ronald G. Gregg ${ }^{2,3}$ and Kirill A. Martemyanov ${ }^{1}$

${ }^{1}$ From the Department of Neuroscience, The Scripps Research Institute, Jupiter, FL 33458 USA; ${ }^{2}$ Department Biochemistry and Molecular Biology and ${ }^{3}$ Ophthalmology and Visual Sciences, University of Louisville, Louisville, KY, 40202, USA

\section{*Address correspondence to:}

Dr. Kirill Martemyanov

Department of Neuroscience, The Scripps Research Institute 130 Scripps Way Jupiter, FL 33458

Phone: (561) 228-2770

Email: kirill@scripps.edu

\section{Abstract}

The extent and temporal characteristics of the GPCR signaling is shaped by the Regulator of $\mathrm{G}$ protein Signaling (RGS) proteins that promote $\mathrm{G}$ protein deactivation. With hundreds of the GPCRs and dozens of RGS proteins, compartmentalization plays key role in establishing signaling specificity. However, the molecular details and mechanisms of this process are poorly understood. Here we report that the R7 group of RGS regulators is controlled by the interaction with previously uncharacterized orphan 
GPCRs: GPR158 and GPR179. We show that GPR158/179 recruit RGS complexes to the plasma membrane and augment their ability to regulate GPCR signaling. The loss of GPR179 in a mouse model of night blindness prevents targeting of RGS to the postsynaptic compartment of bipolar neurons in the retina, illuminating the role of GPR179 in night vision. We propose that the interaction of RGS proteins with orphan GPCRs serves as a mechanism that aids establishing signaling selectivity in G protein pathways.

\section{Introduction}

Signal transduction via heterotrimeric $\mathrm{G}$ proteins is fundamental for mediating a wide range of the cellular responses to changes in the extracellular environment (Offermanns, 2003). In these pathways, the signaling is initiated upon binding of ligand to a $G$ protein coupled receptor (GPCR) that catalyzes the GDP/GTP exchange on the $G$ protein, which leads to their dissociation into active Ga-GTP and Gbg subunits. Control of the kinetics and extent of the signaling in the $G$ protein pathways is realized through the action of the RGS proteins that inactivate the signaling by promoting the GTP hydrolysis on G protein a subunits (Hollinger and Hepler, 2002; Ross and Wilkie, 2000). In mammalian nervous systems, the R7 family of RGS proteins (RGS6, RGS7, RGS9 and RGS11) play key roles in synaptic transmission, light perception and sensitivity to addictive drugs by regulating several GPCR pathways (Anderson et al., 2009; Slepak, 2009). The function of the R7 RGS proteins depends on the formation of the macromolecular complexes with other proteins that dictate their catalytic activity and compartmentalization and allows achieving signaling specificity. Two homologous membrane-anchoring subunits have been previously shown to form complexes with R7 RGS proteins: RGS9 anchor protein (R9AP) and R7 Binding Protein (R7BP) (Jayaraman et al., 2009). Knockout of R9AP or R7BP in mice has been shown to dramatically affect the localization and expression of RGS9 and RGS11 (Anderson et al., 2007a; Cao et al., 
2009; Keresztes et al., 2004). However, the protein levels of RGS6 and RGS7 were not affected upon the elimination of R7BP and only minor changes in the membrane recruitment of these proteins was observed in neurons lacking R7BP (Anderson et al., 2007a; Cao et al., 2008; Panicker et al., 2010). These observations suggest the presence of other, yet unidentified membrane anchor(s) for R7 RGS proteins. However, homology searches of genomic sequences revealed no proteins with sufficient similarity to R7BP/R9AP.

In this report we used an unbiased proteomic approach to identify additional membrane anchors for RGS7 in the nervous system. We demonstrate that the previously uncharacterized orphan GPCRs, GPR158 and GPR179, control localization and activity of RGS7/Gb5 complexes both in reconstituted cells and in vivo. These findings for the first time describe the role of orphan GPCRs GPR158 and GPR179 in the regulation of $\mathrm{G}$ protein signaling.

\section{Results and Discussion}

\section{Identification of GPR158 as a binding partner of RGS7 in the brain}

We conducted an unbiased screen aimed at identifying novel binding partners of RGS7. RGS7 was immunoprecipitated from the total brain lysates followed by the massspectrometric sequencing of pulled down proteins. Gb5 knockout mice, which show dramatically reduced expression of RGS7 (Chen et al., 2003), were used as a negative control to exclude non-specific interactions. We found only 2 proteins with confidence similar to RGS7 (Fig. 1A). The first protein was Gb5, a well-known binding partner of RGS7, validating our identification strategy. The second protein was identified as an orphan G protein coupled receptor 158, or GPR158 (Fig. 1B; Supplemental Table 1). Tandem mass-spectrometry analysis of the identified peptides revealed high confidence of sequence assignment (Supplemental Fig. S1). 
Based on amino acid sequence similarity, GPR158 belongs to the class C GPCR family (Bjarnadottir et al., 2005). Our bioinformatics analysis indicates that the GPR158 (accession NP_065803.2) is conserved across multiple species and contains several conserved residues in the intracellular face of the TM3 and TM6 (Fig. 1 B). However, GPR158 lacks the extracellular venus-flytrap module that plays an essential role in ligand binding and receptor activation in all known class $\mathrm{C}$ receptors (Bjarnadottir et al., 2005; Jingami et al., 2003). Instead, GPR158 features two other conserved elements that are not found in typical class C receptors: a calcium binding EGF-like domain (aa 314-359) and leucine repeat region (aa 108-136). The expression of GPR158 is detected in many tissues, but the protein is particularly prominent in the nervous system (Fig. 1C).

\section{GPR158 specifically interacts with RGS7/Gb5 complex and competes with R7BP}

To validate the interaction of RGS7 with GPR158 we first verified their coimmunoprecipitation from the brain lysates by Western blotting. As illustrated in Fig. 1D precipitation of RGS7 pulls down GPR158 from wild type but not from Gb5 knockout tissues confirming the specificity of the interaction. Conversely, antibodies against GPR158 but not non-immune IgG effectively co-precipitate RGS7 from the brain (Fig. 1D). We next examined interaction between GPR158 and RGS7 in transfected HEK293. Immunoprecipitation of GPR158 by the engineered affinity myc-tag resulted in efficient pull-down of RGS7 together with Gb5 when the proteins were co-expressed (Fig. 1E). Similarly, reciprocal immunoprecipitation of HA-tagged-RGS7 led to co-precipitation of GPR158 when both proteins were present in the cells (Fig. 1E).

Since RGS7 shares high degree of similarity with other R7 RGS proteins we tested if GPR158 interacts with RGS6/Gb5, RGS9-2/Gb5 and RGS11/Gb5 complexes. We found that GPR158 could only co-precipitate with RGS6 but not with RGS9-2 or 
RGS11 (Fig. 1F). Although interaction with RGS6 was specific, it was apparently less efficient relative to RGS7 binding.

RGS7/Gb5 dimer has been previously shown to bind membrane anchor R7BP (Drenan et al., 2005; Martemyanov et al., 2005). Therefore, we next explored whether RGS7/Gb5 can simultaneously bind to both GPR158 and R7BP. Co-immunoprecipitation studies following the reconstitution in HEK293 cells show that the interaction of RGS7 with GPR158 and R7BP is mutually exclusive (Fig. 1G). R7BP pulls down only RGS7 but not GPR158. Conversely, GPR158 pulls down RGS7 but not R7BP. This mutually exclusive nature of R7BP and GPR158 binding to RGS7/Gb5 was further confirmed in the competition experiments (Fig. 1H). The interaction of RGS7 with GPR158 was progressively reduced upon increase in R7BP expression. Similarly, the binding of RGS7 to R7BP decreased when more GPR158 was supplied to the cells. 
A

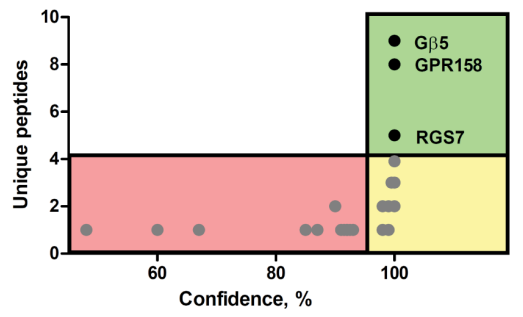

C

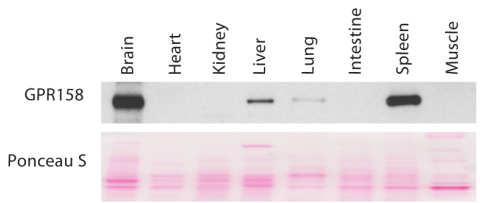

D

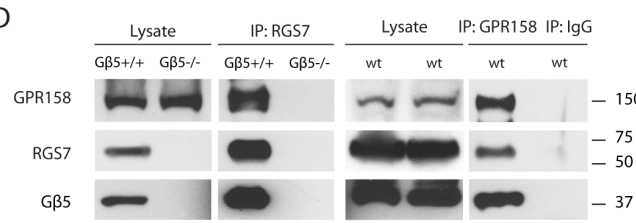

$\mathrm{E}$

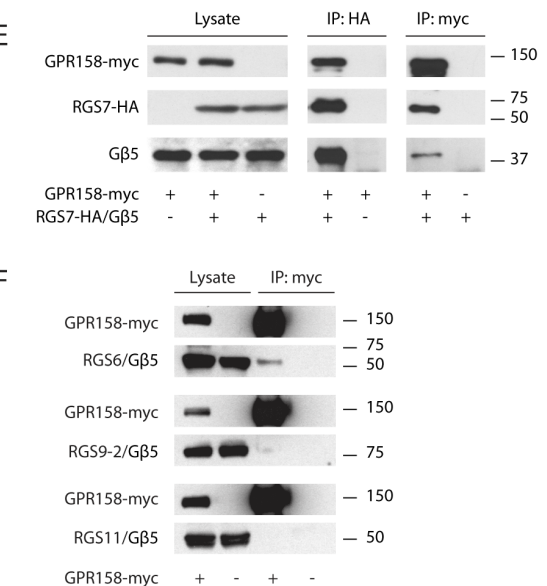

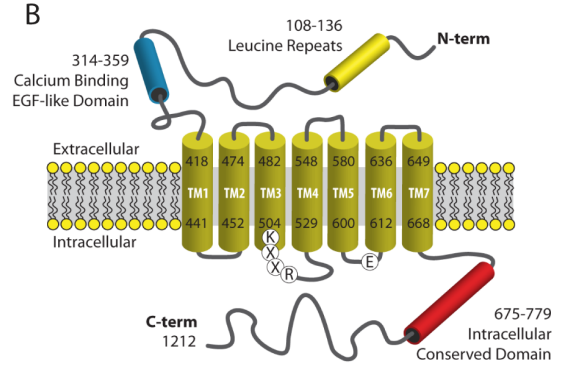

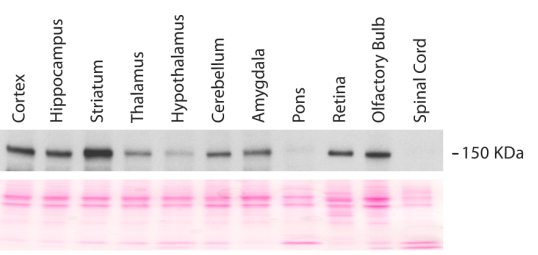

G

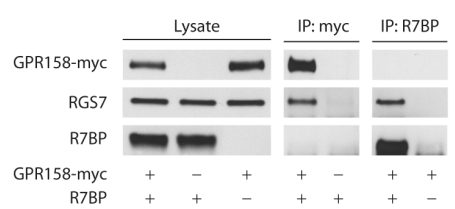

$\mathrm{H}$

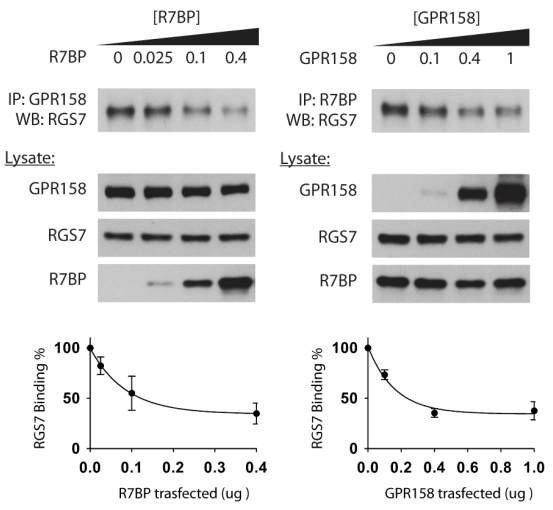

Figure 1. GPR158 is a novel binding partner of RGS7. A, Summary of the massspectrometric analysis of proteins present in the eluates from the preparative immunoprecipitation reaction (IP) using RGS7 antibodies. Positive identification criteria were set to $95 \%$ confidence. Yellow field shows hits above this threshold while red- below. In addition, only proteins with the number of identified unique peptides similar to the target protein (RGS7) were considered (green field). B, Bioinformatic analysis of GPR158 organization. Predicted transmembrane domains and conserved features are depicted as cylinders. Cytoplasmic surface of GPR158 features conservation of key residues important for the G protein activation in class C GPCRs, including Lys 502 and Arg 505 at the cytoplasmic end of the TM3 and Glu 609 in the third intracellular loop. C, Regional specificity of GPR158 expression as evidenced by Western blotting analysis of tissue lysates. D, RGS7 and GPR158 co-immunoprecipitate from native brain lysates. Whole brain tissue was extracted and used for the immunoprecipitation experiments using either RGS7 or GPR158 specific antibodies. E, RGS7 and GPR158 form complex upon co-expression in HEK293 cells. Cells were transfected with the indicated 
constructs and proteins were immunoprecipitated using antibodies directed against affinity tags. F, GPR158 binds to RGS6 but not to RGS9 or RGS11. GPR158 was co-transfected into HEK293 cells with indicated RGS constructs, and coprecipitating RGS proteins were revealed by Western blotting. G, GPR158 does not co-immunoprecipitate with R7BP in the presence of RGS7/Gb5 complex in transfected HEK293 cells. H, GPR158 and R7BP compete for binding to RGS7. Transfection of increasing amounts of R7BP reduced co-immunoprecipitation of GPR158 with RGS7 and conversely, increasing concentrations of GPR158 reduced binding of RGS7 to R7BP. 


\section{GPR158 targets RGS7/Gb5 complex to the plasma membrane via the interaction with the DEP domain}

Since GPR158 is a membrane protein we next asked whether it can change the localization of RGS7 complex in the cells. We found that when expressed in HEK293 cells, GPR158 is efficiently targeted to the plasma membrane (Fig. 2A). In contrast, as previously noted (Drenan et al., 2006; Zhang et al., 2001), localization of RGS7/Gb5 was mostly cytoplasmic. However, co-expression of RGS7/Gb5 with GPR158 resulted in its efficient translocation to the plasma membrane (Fig. 2A). We further confirmed recruitment of RGS7/Gb5 to the plasma membrane biochemically (Fig. 2B). Consistent with the immunocytochemistry data, most of RGS7 was found in the cytosolic fraction upon sedimentation analysis but moved to the membrane pellet when co-expressed with GPR158 (Fig. 2B). These results suggest that GPR158 serves as a membrane anchor for RGS7/Gb5 complex. 


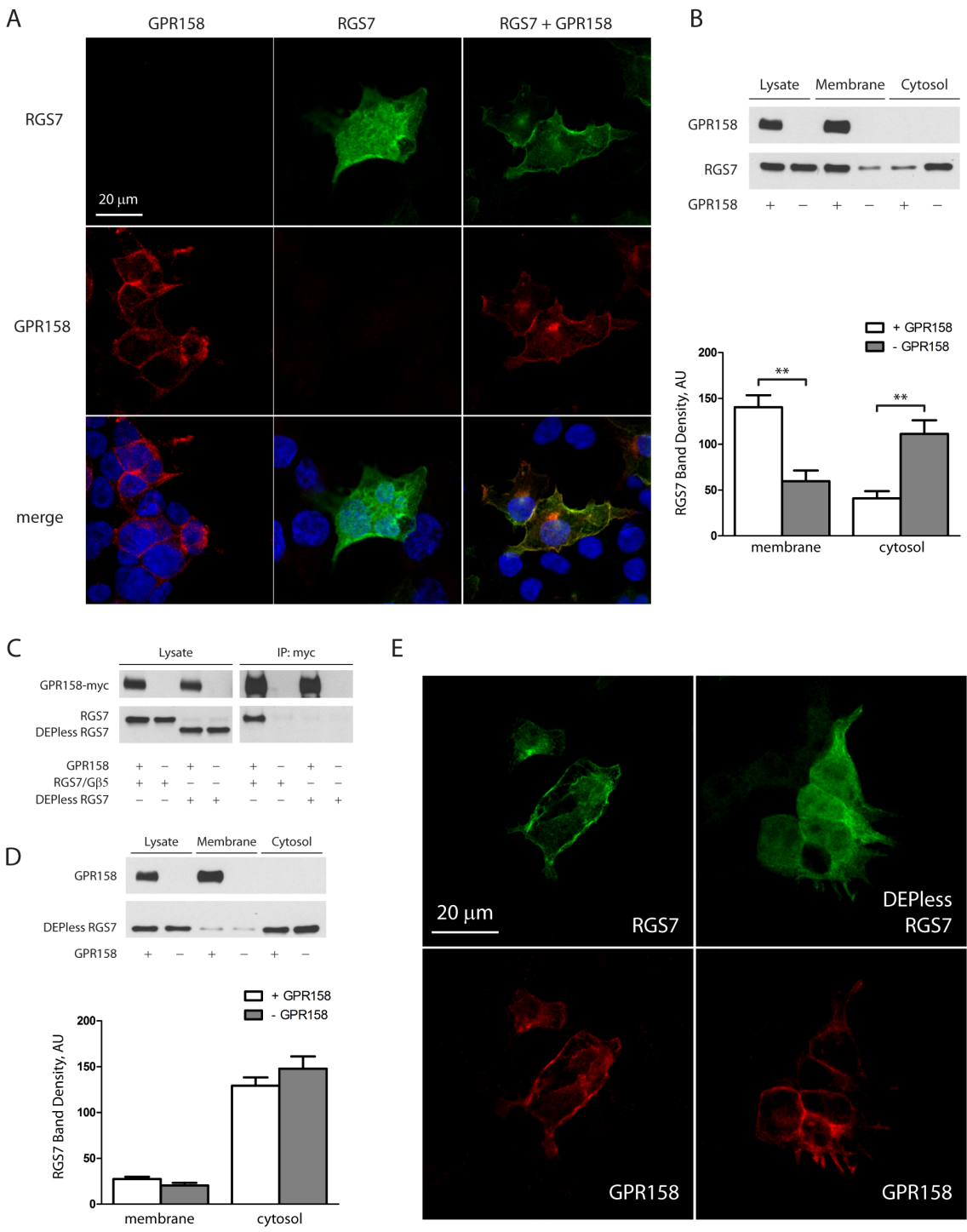

Figure 2. GPR158 recruits RGS7 to the plasma membrane in a DEP domain dependent manner. A, Analysis of GPR158 and RGS7 localization in transfected HEK293 cells by immunocytochemistry followed by confocal microscopy. RGS7/Gb5 and GPR158 were expressed alone or together and their localization was examined. B, RGS7 fractionates with the plasma membrane fraction in the presence of GPR158. Following transfection cells were disrupted and fractionated by sedimentation into membrane and cytosol portions. Band densities were quantified from 3 independently conducted experiments. ${ }^{* *}, p<0.01$, t-test. C, fulllength RGS7, but not RGS7 mutant with the deleted DEP domain (DEPless RGS7) binds to GPR158. HEK293 cells were co-transfected with the indicated constructs and interaction of proteins was studied by the immunoprecipitation. D, DEPless RGS7 does not co-segregate with GPR158 in the membrane fraction upon sedimentation analysis. E, DEPless RGS7 fails is not recruited to the plasma membrane of the transfected HEK293 cells that express GPR158. Cells were cotransfected with indicated constructs and protein localization was studied by immunostaining. 
Competition between GPR158 and R7BP for binding to RGS7 suggests that their interactions are mediated by the same or overlapping determinants. Since binding to R7BP requires the presence of the DEP domain of RGS7 (Anderson et al., 2009) we hypothesized that this domain also mediates the interaction of RGS7 with GPR158. Indeed, a truncated mutant of RGS7 lacking the DEP domain (DEPless), while preserving the interaction with G 35 did not co-immunoprecipitate with GPR158 upon cotransfection in HEK293 cells (Fig. 2C). Consequently, DEPless-RGS7 failed to be recruited to the plasma membrane by GPR158 as evidenced by either biochemical fractionation (Fig. 2D) or immunocytochemistry (Fig. 2E).

\section{GPR158 augments GAP activity of RGS7 towards Gao}

The identification of the novel interaction of RGS7/Gb5 complex with GPR158 raises the question about its physiological significance. Members of the R7 RGS family are efficient GAPs for the Go class of the proteins, downstream from multiple GPCRs including m-opioid receptor (Anderson et al., 2009). We therefore used a cell based BRET assay to monitor the effects of RGS7/Gb5 on Gao activated by the m-opioid receptor (Fig. 3A; (Hollins et al., 2009). We primarily focused on analyzing the deactivation kinetics of $G$ protein signaling that reflect the catalytic activity of RGS proteins. Consistent with the previous reports, we found that RGS7/Gb5 complex accelerated Gao deactivation kinetics (Fig. 3B). Co-transfection of GPR158 resulted in further acceleration of signaling termination. Notably, GPR158 did not influence the kinetics of $\mu$-opioid signaling when supplied without RGS7/Gb5 complex, indicating that it acts via increasing the activity of RGS7 (Fig. 3C). Indeed, the catalytic activity of RGS7/Gb5 as measured by the $k_{\text {GAP }}$ parameter was increased by GPR158 by $\sim 2$ fold (from $0.081 \pm 0.016 \mathrm{~s}^{-1}$ to $0.152 \pm 0.022 \mathrm{~s}^{-1}$ ). We found no effect of GPR158 on either the activation kinetics of Gao that reflects MOR activity in the cells (Fig. 3D) or the expression of reporter constructs (Supplemental Figure S2). Furthermore, no 
significant effect of GPR158 on the expression of RGS7 was detected under the conditions and concentrations of components used for the BRET assays (Fig. 3E). This suggests that GPR158 exerts direct stimulatory effect the activity of RGS7/Gb5 complex. 

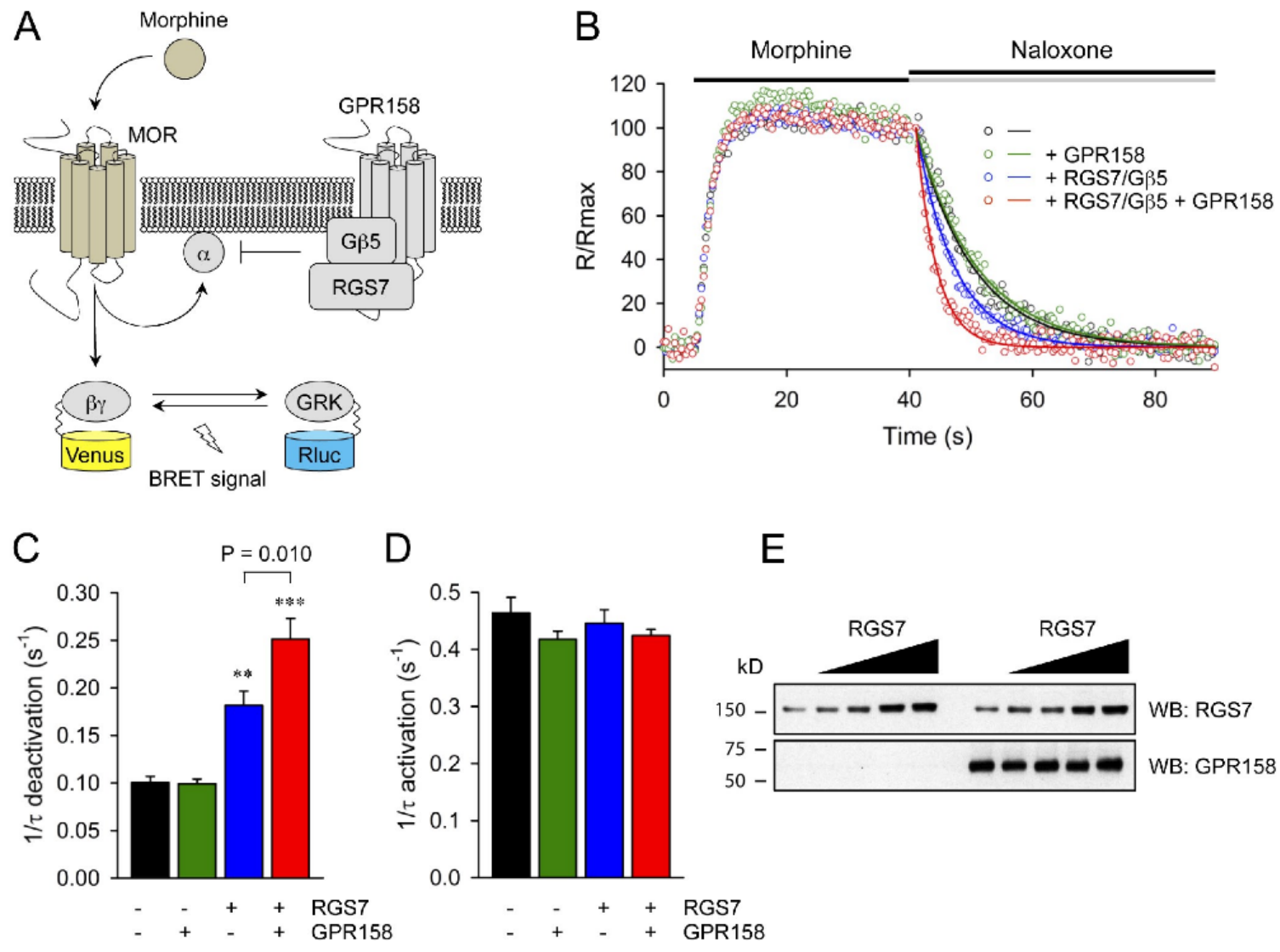

Figure 3. GPR158 potentiates the ability of RGS7/Gb5 to deactivate Gao signaling. $A$, Schematic representation of the BRET-based assay to monitor $G$ protein signaling cycle. Activation of the $m$-opioid receptor (MOR) causes the $G$ protein heterotrimer to dissociate into $\mathrm{Ga}$ and $\mathbf{G b g}$ subunits. Released $\mathbf{G b g}$ subunits tagged with Venus fluorescent protein interact with luciferase (Rluc) -tagged reporter GRK to produce BRET signal. Upon termination of MOR activation by antagonist naloxone, Gao subunit hydrolyses GTP and re-associates with Gbg subunits quenching the BRET signal. $B$, Time-course of the normalized BRET responses recorded in a representative experiment. Individual data points show BRET values averaged from 6 replicates. Application of MOR agonist (morphine) and antagonist (naloxone) is indicated by the upper bars. Deactivation phase of the response was fitted with the single exponent (solid line). C, Quantification of the deactivation time constant following the addition of naltrexone. Exponential fits of the data shown in panel $B$ were used to derive time constant $t$. Asterisks indicate statistical significance of the differences $\left({ }^{* *}, p<0.01\right.$ and ${ }^{* * *}, p<0.001$; Mann-Whitney Rank Sum Test; $n=18$ ) as compared to control experiment with no regulators added (black bar). D, Quantification of the activation time constant derived from the exponential fitting of the onset kinetics (fits not shown). Note no significant differences between experimental conditions. E, Analysis of the GPR158 effect on expression level of RGS7. The HEK293 cells were transfected with all constructs used for the BRET experiments using the same ratios and conditions. Proteins of interest were detected by Western blotting using specific antibodies. 


\section{GPR158-like protein, GPR179 targets RGS/Gb5 complexes to the dendritic tips of ON-bipolar cells}

Regulation of RGS7/Gb5 complex localization and activity by GPR158 prompted us to ask whether the interaction with the GPCR-like proteins is a general mechanism for controlling RGS7 function in neurons and whether proteins similar to GPR158 might also be engaged in this process. Our analysis shows that GPR158 shares substantial sequence similarity with another orphan receptor GPR179 (Fig 4A). We found that GPR179 (accession NP_001004334.2), just like GPR158, also forms specific complexes with RGS7 (Fig. 4B). However, unlike GPR158, it could interact with all members of the R7 RGS subfamily (Fig. 4C). Interestingly, GPR179 exhibited much more restricted expression and was detected only in the retina (Fig. 4D). Two recent studies showed that mutations in GPR179 gene cause congenital stationary night blindness (CSNB) in humans indicating GPR179 is required for normal synaptic transmission between photoreceptors and ON-bipolar cells in the retina (Audo et al., 2012; Peachey et al., 2012). Furthermore, a mouse mutant nob5 lacks an ERG b-wave and is a model for this form of cCSNB (Peachey et al., 2012). Earlier studies showed that RGS7/Gb5 and RGS11/Gb5 complexes co-localize with the essential components of the signaling cascade at the dendritic tips of the ON-bipolar cells and play an important role in normal transmission at this synapse (Chen et al., 2010; Mojumder et al., 2009; Zhang et al., 2010). We therefore asked whether GPR179 could influence localization and function of RGS proteins in these neurons. Indeed, our examination revealed that RGS7 and RGS11 co-localizes with GPR179 at the dendritic tips of the ON-bipolar cells (Fig. 4E). Remarkably, loss of GPR179 in the nob5 mice resulted in loss of the punctate staining for both RGS7 and RGS11 (Fig. 4 G,H), although their protein levels were unchanged (Fig. 4F). These data suggest that GPR179 is essential for the postsynaptic targeting of the RGS/Gb5 complexes in retinal ON-bipolar neurons. 

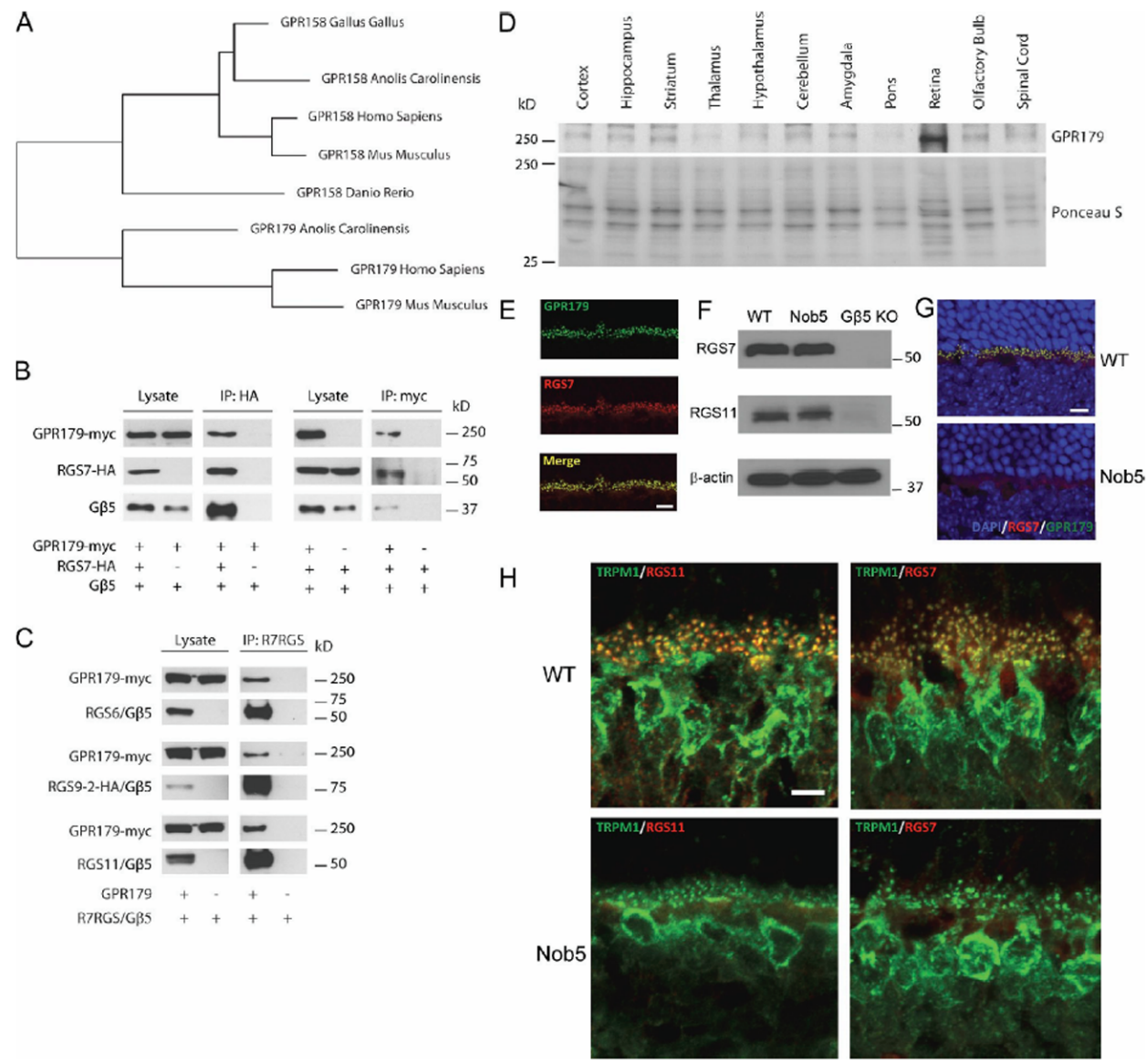

Figure 4. GPR179 is a paralogue of GPR158 required for subcellular targeting of RGS7/Gb5 complex in vivo. A, GPR158 shares considerable sequence homology and conservation among species with GPR179 as revealed by phylogenic analysis. B, GPR179 forms complexes with RGS7 in transfected cells. Forward and reverse immunoprecipitation experiments were carried using indicated antibodies following co-transfection of GPR158 with RGS7 in HEK293 cells. C, Interaction of GPR179 with members of the R7 RGS subfamily. RGS/Gb5 complexes were co-expressed with GPR179 in HEK293 cells and the interactions between proteins were studied by the co-immunoprecipitation assays. D, Expression profile of GPR179 across nervous tissues as determined by the Western blotting of total tissue lysates. Ponceau $\mathbf{S}$ staining for total protein indicates equal sample loading. E, GPR179 co-localizes with RGS7 and RGS11 at the dendritic tips of the ON-bipolar cells in the outer plexiform layer of the retina. Retina cross-sections were immunolabeled for GPR179 (green) and RGS7 (red). Note characteristic punctate pattern of staining that indicates synaptic localization of both proteins. F, Loss of GPR179 in nob5 retinas does not affect the expression of RGS7 and RGS11. Western blot analysis of RGS7 and RGS11 expression in total retina lysates from wild type mice (WT) or mice lacking GPR179 (nob5). G and $H$, Elimination of GPR179 prevents targeting of RGS7 and RGS11 but not TRPM1 to the dendritic tips. Retina cross-sections were double immunostained for RGS7 (red) and GPR179 in panel G or RGS7/RGS11 (red) and TRPM1 (green) in panel H. Cell nuclei are labeled with DAPI (blue). 


\section{GPR158/179 and G protein signaling}

The results of our study reveal the existence of a new family of membrane anchors and activity modulators for the R7 RGS proteins that belong to the group of orphan GPCRs and contain two members GPR158 and GPR179. Two other membrane anchors for the R7 RGS family have been described previously (Jayaraman et al., 2009). R9AP was found to form complexes with RGS9 and RGS11 but not with RGS6 or RGS7 and was demonstrated to play key role in controlling posttranslational stability, subcellular targeting and activity of its RGS partners (Anderson et al., 2009; Jayaraman et al., 2009). Subsequently, R9AP homologue, R7BP, was identified as universal partner for all members of the R7 RGS subfamily (Drenan et al., 2005; Martemyanov et al., 2005). Similarly to R9AP, R7BP was demonstrated to play role in stabilization, localization and activity regulation for some, but not all R7 RGS proteins in some, but not all neurons (Anderson et al., 2009; Jayaraman et al., 2009). For example, R7BP was shown to be important for achieving the high expression level of RGS9-2 in the striatum, but did not affect the expression of RGS7 in the same region (Anderson et al., 2007a). Likewise, while R7BP is important for the recruitment of RGS7/Gb5 complex to the nucleus (Panicker et al., 2010), it was not required for the delivery of the same complex to the dendritic tips of the ON-bipolar neurons in the retina (Cao et al., 2008) and only mildly affected RGS7/Gb5 recruitment to the plasma membrane in the brain (Panicker et al., 2010). These observations suggested the existence of additional targeting mechanisms for the R7 RGS complexes, particularly for the RGS7/Gb5. The identification of GPR158 and GPR179 as RGS membrane anchors, suggests a new mechanism for achieving subcellular targeting of this important class of GPCR regulatory proteins.

Our findings provide a new example for the interaction between R7 RGS proteins with the GPCRs. Previous studies found that RGS9 can form complexes with m-opioid 
(Garzon et al., 2005) and D2 dopamine receptors (Kovoor et al., 2005). Furthermore, RGS7/Gb5 complexes have been shown to bind to the third intracellular loop of M3 muscarinic receptor, via a direct protein-protein interaction involving the DEP domain of the molecule (Sandiford et al., 2010). As in case of the GPR158, this binding was mutually exclusive with R7BP recruitment, and R7BP prevented the interaction. Similarly, the DEP domain of the primordial yeast RGS protein Sst2 mediates its recruitment to the pheromone sensing GPCR Ste2 (Ballon et al., 2006). These observations indicate that in addition to specialized membrane anchoring subunits, the DEP domains of R7RGS proteins are recruited to elements found in some GPCRs. Yet, no common motifs are detected across any DEP domain interacting proteins reinforcing an idea that these modules potentially recognize a diverse set of targets.

The interaction with GPR158 results in translocation of RGS7/Gb5 complexes to the plasma membrane compartments and augmentation of their catalytic activity. In the case retinal ON-bipolar cells, GPR179 is required for the localization of both RGS7 and RGS11. Since these RGS proteins in complex with the Gb5 are essential for the synaptic transmission at the ON-bipolar synapse (Chen et al., 2003), their mistargeting in mice with GPR179 deletion or human patients with mutations in GPR179 gene (Audo et al., 2012; Peachey et al., 2012) may account for their no b-wave phenotype and night blindness, respectively. What remains unexplored however is whether GPR158 and GPR179 only serve as RGS anchor proteins, or whether they can act as bona fide GPCRs. Both are distant members of the class C GPCRs (Bjarnadottir et al., 2005) and our bioinformatics analysis shows that the amino acids that are critical for the ability of the class $C$ receptors to activate $G$ proteins (Binet et al., 2007) also are conserved in GPR158 and GPR179. This suggests a possibility that these orphan receptors can, in principle, activate $\mathrm{G}$ proteins. However, the lack of typical class $\mathrm{C}$ ligand binding domain 
in GPR158/GPR179 suggests that if they can in fact activate G proteins the mechanism must be substantially different.

\section{Materials and Methods}

\section{Mice, antibodies and genetic constructs}

Generation of sheep anti-RGS6, sheep anti-RGS9-2 and sheep antiRGS11 antibodies was described (Cao et al., 2008; Martemyanov et al., 2005). Rabbit anti-Gß5, rabbit anti-RGS7 (7RC1) and rabbit anti-R7BP (TRS) were generous gifts from Dr. William Simonds (National Institute of Diabetes and Digestive and Kidney Disease, National Institutes of Health, Bethesda, MD). Mouse anti-HA (Millipore), rabbit anti-myc (Genscript), rabbit anti-GPR179 (Sigma) and rabbit anti-GPR158 (Sigma) were purchased. The GPR179 ${ }^{\text {nob5/nob5 }}$ mice are described elsewhere (Peachey et al., 2012).

Cloning of full-length RGS7, DEPless-RGS7, RGS6, RGS9-2, RGS11, Gß5, R7BP and N-terminal HA-tagged RGS7 in pcDNA3.1/V5-His-TOPO was described (Anderson et al., 2007b; Martemyanov et al., 2003; Martemyanov et al., 2005; Panicker et al., 2010; Porter et al., 2010). Human myc-tagged-GPR158 and myc-tagged-GPR179 were purchased from OriGene. BRET sensor constructs Venus155-239-Gb1, Venus1155-Gg2 and masGRKct-Rluc8 were kindly provided by Dr. Nevin A. Lambert (Medical College of Georgia).

\section{Cell culture, Transfection, Immunoprecipitation and Western blotting}

HEK293T cells were cultured at $37^{\circ} \mathrm{C}$ and $5 \%$ CO2 in DMEM supplemented with $10 \%$ FBS, MEM non-essential amino acids, $1 \mathrm{mM}$ sodium pyruvate, and antibiotics (100 units $/ \mathrm{ml}$ penicillin and $100 \mu \mathrm{g} / \mathrm{ml}$ streptomycin. Cells were transfected using Lipofectamine LTX (Invitrogen), harvested $24 \mathrm{~h}$ later, lysed in ice-cold IP buffer (300 mM $\mathrm{NaCl}, 50 \mathrm{mM}$ Tris-HCl pH 7.4, 1\% Triton X-100 and complete protease inhibitor cocktail) by sonication. For immunoprecipitation, lysates were cleared by centrifugation at 14,000 
rpm for $15 \mathrm{~min}$, and the supernatants were incubated with $20 \mu \mathrm{l}$ of Dynabeads (Invitrogen) and $2 \mu \mathrm{g}$ of antibodies on a rocker at $4^{\circ} \mathrm{C}$ for $1 \mathrm{~h}$. After three washes with IP buffer, proteins were eluted with $50 \mu$ of 4 X SDS sample buffer. Samples were analyzed by SDS-PAGE followed by Western blotting using HRP-conjugated secondary antibodies and an ECL West Pico (Thermo Scientific) detection system.

\section{Preparative immunoprecipitation and mass spectrometry}

Whole brains were removed from mice, homogenized in IP buffer (1xPBS, 0.3 $\mathrm{mM} \mathrm{NaCl}, 1 \%$ Triton $\mathrm{X}-100$, protease inhibitor cocktail (Roche)) with a series of needles with increasing gauge and lysed for $30 \mathrm{~min}$ at $+4{ }^{\circ} \mathrm{C}$. Samples were centrifuged $(30,000$ $\mathrm{g}, 20 \mathrm{~min}$ ) to remove debris, and supernatants were incubated for $45 \mathrm{~min}$ with $5 \mathrm{mg}$ of anti-RGS7 antibody covalently coupled to Protein G beads as previously described (Martemyanov et al., 2005). Protein complexes were eluted with $5 \%$ ammonium hydroxide and vacuum-dried.

Proteins were dissolved in $0.5 \mathrm{M}$ triethylammonium bicarbonate $(\mathrm{pH}$ 8.5) containing $0.1 \%$ SDS, reduced with $5 \mathrm{mM}$ tris-(2-carboxyethyl) phosphine for $1 \mathrm{~h}$ at 60 ${ }^{\circ} \mathrm{C}$ and alkylated with $10 \mathrm{mM}$ methyl methanethiosulfonate for $10 \mathrm{~min}$ at room temperature. Proteins were digested with $20 \mu \mathrm{g}$ of modified porcine trypsin (Promega) in the presence of $3 \mathrm{mM} \mathrm{CaCl} 2$ at $37{ }^{\circ} \mathrm{C}$ for $16 \mathrm{~h}$. Peptide mixtures were vacuum-dried, reconstituted in $0.2 \%$ formic acid (Pierce, Rockford, IL) and applied to a MCX cartridge (Waters, Milford, MA) pre-equilibrated with methanol/water $(1: 1, v / v)$. The cartridge was washed with $0.1 \%$ formic acid in $5 \%$ methanol, followed by a $100 \%$ methanol wash. Peptides were eluted from the $\mathrm{MCX}$ resin in $1 \mathrm{ml}$ of $1.5 \% \mathrm{NH}_{4} \mathrm{OH}$ in methanol and vacuum-dried. The resulting peptide mixtures were desalted, resolved by liquid chromatography and analyzed by LTQ-Orbitrap XL mass spectrometry as described (Cao et al., 2011).

\section{Membrane/cytosol fractionation}


HEK293T transfected cells were lysed in lysis buffer (50 mM Tris-HCl pH 7.4, 2.5 $\mathrm{mM} \mathrm{MgCl2,} 1 \mathrm{mM}$ EDTA, $1 \mathrm{mM}$ DTT, complete protease inhibitor cocktail) by 10 passages through a 27-gauge needle and then centrifuged at $400 \times \mathrm{g}$ for $5 \mathrm{~min}$ at $4^{\circ} \mathrm{C}$ to remove nuclei and intact cells. Supernatants were ultracentrifuged at $150,000 \times \mathrm{g}$ for 20 $\min$ at $4^{\circ} \mathrm{C}$. The cytosolic soluble fraction was collected from the supernatant while the pellet containing the membrane fraction was resuspended in an equal amount of lysis buffer. Samples were then sonicated and 4X SDS sample buffer added for Western blot analysis.

\section{Immunocytochemistry}

HEK293T transfected cells or retina cross-sections were fixed for 15 min with $4 \%$ paraformaldehyde, permeabilized for 5 min with $0.2 \%$ Triton X-100/PBS, blocked with $10 \%$ donkey serum for $1 \mathrm{~h}$, and incubated with primary antibody in $2 \%$ donkey serum for $1 \mathrm{~h}$. After three washes, sections were incubated with AlexaFluor488 or 546-conjugated secondary antibodies for $1 \mathrm{~h}$. Cells were stained 5 min with DAPI before mounting in Fluoromount (Sigma). Images were taken by the LSM 780 Zeiss confocal microscope.

\section{Monitoring $\mathrm{G}$ protein cycle in live cells by fast kinetic BRET assay}

Agonist-dependent cellular measurements of bioluminescence resonance energy transfer (BRET) between masGRKct-Rluc8 and Gb1g2-Venus were performed to visualize the action of $G$ protein signaling in living cells as previously described with slight modification (Hollins et al., 2009; Lambert et al., 2010). HEK293T/17 were grown in Dulbecco's modified Eagle's medium (DMEM) supplemented with $10 \%$ fetal bovine serum (FBS), MEM non-essential amino acids, $1 \mathrm{mM}$ sodium pyruvate, and antibiotics (100 units $/ \mathrm{mL}$ penicillin and $100 \mu \mathrm{g} / \mathrm{mL}$ streptomycin) at $37^{\circ} \mathrm{C}$ in a humidified incubator containing $5 \% \mathrm{CO}_{2}$. For transfection, cells were seeded into $6-\mathrm{cm}$ dishes at a density of $4 \times 10^{6}$ cells/dish. After $4 \mathrm{~h}$, expression constructs (total $5 \mu \mathrm{g} / \mathrm{dish}$ ) were transfected into the cells using Lipofectamine LTX (8 $\mu \mathrm{L} /$ dish) and PLUS $(5 \mu \mathrm{L} / \mathrm{dish})$ 
reagents. MOR, Gao, Venus155-239-Gb1, Venus1-155-Gg2, masGRKct-Rluc8, RGS7, Gb5, and GPR158 constructs were transfected using equal DNA amounts. Empty vector was used to balance the amount of transfected DNA. The cells were used for experiments at 16-24 $\mathrm{h}$ after transfection. BRET measurements were made using a micro plate reader (POLARstar Omega; BMG Labtech) equipped with two emission photomultiplier tubes, allowing to detect two emissions simultaneously with resolution of 50 milliseconds for every data point. All measurements were performed at room temperature. The BRET signal is determined by calculating the ration of the light emitted by the Gb1g2-Venus (535 nm) over the light emitted by the masGRKct-Rluc8 (475 nm). The average baseline value recorded prior to agonist stimulation was subtracted from BRET signal values, and the resulting difference $(R)$ was normalized against the maximal value (Rmax) recorded upon agonist stimulation.

\section{Statistical analyses}

We used Student's t-test to analyze densitometry data from biochemical fractionation experiments. For the analysis of the non-parametric data reporting differences in the exponential rate constant of the $G$ protein deactivation kinetics observed in BRET experiments we used Mann-Whitney rank-sum test. The confidence values below $p<0.05$ were considered to be statistically significant.

\section{Acknowledgements}

We would like to thank Dr. William Simonds $(\mathrm{NIH})$ for the generous gift of antiGb5 antibodies and DEPless RGS7 construct, and Dr. Nevin A. Lambert (Medical College of Georgia) for the BRET sensor constructs Venus155-239-Gb1, Venus1-155Gg2 and masGRKct-Rluc8. This work was supported by NIH grants DA021743, DA026405, and EY018139 (to KAM); EY012354 (to RGG).

\section{Abbreviations List}

RGS: Regulators of G protein Signaling 
GPCR: G Protein Coupled Receptor

GAP: GTPase Activating Protein

DEP: Disheveled, EGL-10, Pleckstrin homology domain

R7BP: R7 family Binding Protein

R9AP: RGS9 Anchor Protein

\section{References}

Anderson, G.R., R. Lujan, A. Semenov, M. Pravetoni, E.N. Posokhova, J.H. Song, V. Uversky, C.K. Chen, K. Wickman, and K.A. Martemyanov. 2007a. Expression and localization of RGS9-2/Gb5/R7BP complex in vivo is set by dynamic control of its constitutive degradation by cellular cysteine proteases. $J$ Neurosci. 27:14117-14127.

Anderson, G.R., E. Posokhova, and K.A. Martemyanov. 2009. The R7 RGS protein family: multi-subunit regulators of neuronal $G$ protein signaling. Cell Biochem Biophys. 54:33-46.

Anderson, G.R., A. Semenov, J.H. Song, and K.A. Martemyanov. 2007b. The membrane anchor R7BP controls the proteolytic stability of the striatal specific RGS protein, RGS9-2. J Biol Chem. 282:4772-4781.

Audo, I., K. Bujakowska, E. Orhan, C.M. Poloschek, S. Defoort-Dhellemmes, I. Drumare, S. Kohl, T.D. Luu, O. Lecompte, E. Zrenner, M.E. Lancelot, A. Antonio, A. Germain, C. Michiels, C. Audier, M. Letexier, J.P. Saraiva, B.P. Leroy, F.L. Munier, S. Mohand-Said, B. Lorenz, C. Friedburg, M. Preising, U. Kellner, A.B. Renner, V. Moskova-Doumanova, W. Berger, B. Wissinger, C.P. Hamel, D.F. Schorderet, E. De Baere, D. Sharon, E. Banin, S.G. Jacobson, D. Bonneau, X. Zanlonghi, G. Le Meur, I. Casteels, R. Koenekoop, V.W. Long, F. Meire, K. Prescott, T. de Ravel, I. Simmons, H. Nguyen, H. Dollfus, O. Poch, T. Leveillard, K. Nguyen-Ba-Charvet, J.A. Sahel, S.S. Bhattacharya, and C. Zeitz. 2012. Whole-Exome Sequencing Identifies Mutations in GPR179 Leading to Autosomal-Recessive Complete Congenital Stationary Night Blindness. Am J Hum Genet. 90:321-330.

Ballon, D.R., P.L. Flanary, D.P. Gladue, J.B. Konopka, H.G. Dohlman, and J. Thorner. 2006. DEP-domain-mediated regulation of GPCR signaling responses. Cell. 126:1079-1093.

Binet, V., B. Duthey, J. Lecaillon, C. Vol, J. Quoyer, G. Labesse, J.P. Pin, and L. Prezeau. 2007. Common structural requirements for heptahelical domain function in class A and class C G protein-coupled receptors. J Biol Chem. 282:12154-12163.

Bjarnadottir, T.K., R. Fredriksson, and H.B. Schioth. 2005. The gene repertoire and the common evolutionary history of glutamate, pheromone (V2R), taste(1) and other related G protein-coupled receptors. Gene. 362:70-84.

Cao, Y., I. Masuho, H. Okawa, K. Xie, J. Asami, P.J. Kammermeier, D.M. Maddox, T. Furukawa, T. Inoue, A.P. Sampath, and K.A. Martemyanov. 2009. Retina-specific GTPase accelerator RGS11/Gb5S/R9AP is a constitutive heterotrimer selectively targeted to mGluR6 in ON-bipolar neurons. J Neurosci. 29:9301-9313. 
Cao, Y., E. Posokhova, and K.A. Martemyanov. 2011. TRPM1 forms complexes with nyctalopin in vivo and accumulates in postsynaptic compartment of ON-bipolar neurons in mGluR6-dependent manner. J Neurosci. 31:11521-11526.

Cao, Y., H. Song, H. Okawa, A.P. Sampath, M. Sokolov, and K.A. Martemyanov. 2008. Targeting of RGS7/Gb5 to the dendritic tips of ON-bipolar cells is independent of its association with membrane anchor R7BP. J Neurosci. 28:10443-10449.

Chen, C.K., P. Eversole-Cire, H.K. Zhang, V. Mancino, Y.J. Chen, W. He, T.G. Wensel, and M.I. Simon. 2003. Instability of GGL domain-containing RGS proteins in mice lacking the G protein b-subunit Gb5. Proc Natl Acad Sci U S A. 100:6604-6609.

Chen, F.S., H. Shim, D. Morhardt, R. Dallman, E. Krahn, L. McWhinney, A. Rao, S.J. Gold, and C.K. Chen. 2010. Functional redundancy of R7 RGS proteins in ONbipolar cell dendrites. Invest Ophthalmol Vis Sci. 51:686-693.

Drenan, R.M., C.A. Doupnik, M.P. Boyle, L.J. Muglia, J.E. Huettner, M.E. Linder, and K.J. Blumer. 2005. Palmitoylation regulates plasma membrane-nuclear shuttling of R7BP, a novel membrane anchor for the RGS7 family. J Cell Biol. 169:623633.

Drenan, R.M., C.A. Doupnik, M. Jayaraman, A.L. Buchwalter, K.M. Kaltenbronn, J.E. Huettner, M.E. Linder, and K.J. Blumer. 2006. R7BP augments the function of RGS7/Gb5 complexes by a plasma membrane-targeting mechanism. J Biol Chem. 281:28222-28231.

Garzon, J., M. Rodriguez-Munoz, A. Lopez-Fando, and P. Sanchez-Blazquez. 2005. Activation of mu-opioid receptors transfers control of Galpha subunits to the regulator of G-protein signaling RGS9-2: role in receptor desensitization. J Biol Chem. 280:8951-8960.

Hollinger, S., and J.R. Hepler. 2002. Cellular regulation of RGS proteins: Modulators and integrators of $G$ protein signaling. Pharmacol Rev. 54:527-559.

Hollins, B., S. Kuravi, G.J. Digby, and N.A. Lambert. 2009. The c-terminus of GRK3 indicates rapid dissociation of $G$ protein heterotrimers. Cell Signal. 21:1015-1021.

Jayaraman, M., H. Zhou, L. Jia, M.D. Cain, and K.J. Blumer. 2009. R9AP and R7BP: traffic cops for the RGS7 family in phototransduction and neuronal GPCR signaling. Trends Pharmacol Sci. 30:17-24.

Jingami, H., S. Nakanishi, and K. Morikawa. 2003. Structure of the metabotropic glutamate receptor. Curr opinion neurobiol. 13:271-278.

Keresztes, G., K.A. Martemyanov, C.M. Krispel, H. Mutai, P.J. Yoo, S.F. Maison, M.E. Burns, V.Y. Arshavsky, and S. Heller. 2004. Absence of the RGS9/Gb5 GTPaseactivating Complex in Photoreceptors of the R9AP Knockout Mouse. J Biol Chem 279:1581-1584.

Kovoor, A., P. Seyffarth, J. Ebert, S. Barghshoon, C.K. Chen, S. Schwarz, J.D. Axelrod, B.N. Cheyette, M.I. Simon, H.A. Lester, and J. Schwarz. 2005. D2 dopamine receptors colocalize regulator of G-protein signaling 9-2 (RGS9-2) via the RGS9 DEP domain, and RGS9 knock-out mice develop dyskinesias associated with dopamine pathways. J Neurosci. 25:2157-2165.

Lambert, N.A., C.A. Johnston, S.D. Cappell, S. Kuravi, A.J. Kimple, F.S. Willard, and D.P. Siderovski. 2010. Regulators of G-protein signaling accelerate GPCR signaling kinetics and govern sensitivity solely by accelerating GTPase activity. Proc Natl Acad Sci U S A. 107:7066-7071.

Martemyanov, K.A., P.V. Lishko, N. Calero, G. Keresztes, M. Sokolov, K.J. Strissel, I.B. Leskov, J.A. Hopp, A.V. Kolesnikov, C.K. Chen, J. Lem, S. Heller, M.E. Burns, and V.Y. Arshavsky. 2003. The DEP domain determines subcellular targeting of the GTPase activating protein RGS9 in vivo. J Neurosci. 23:10175-10181. 
Martemyanov, K.A., P.J. Yoo, N.P. Skiba, and V.Y. Arshavsky. 2005. R7BP, a novel neuronal protein interacting with RGS proteins of the R7 family. $J$ Biol Chem. 280:5133-5136.

Mojumder, D.K., Y. Qian, and T.G. Wensel. 2009. Two R7 regulator of G-protein signaling proteins shape retinal bipolar cell signaling. J Neurosci. 29:7753-7765.

Offermanns, S. 2003. G-proteins as transducers in transmembrane signalling. Prog Biophys Mol Biol. 83:101-130.

Panicker, L.M., J.H. Zhang, E. Posokhova, M.J. Gastinger, K.A. Martemyanov, and W.F. Simonds. 2010. Nuclear localization of the G protein b5/R7-regulator of $G$ protein signaling protein complex is dependent on R7 binding protein. J Neurochem.

Peachey, N.S., T.A. Ray, R. Florijn, L.B. Rowe, T. Sjoerdsma, S. Contreras-Alcantara, K. Baba, G. Tosini, N. Pozdeyev, P.M. luvone, P. Bojang, Jr., J.N. Pearring, H.J. Simonsz, M. van Genderen, D.G. Birch, E.I. Traboulsi, A. Dorfman, I. Lopez, H. Ren, A.F. Goldberg, P.M. Nishina, P. Lachapelle, M.A. McCall, R.K. Koenekoop, A.A. Bergen, M. Kamermans, and R.G. Gregg. 2012. GPR179 Is Required for Depolarizing Bipolar Cell Function and Is Mutated in Autosomal-Recessive Complete Congenital Stationary Night Blindness. Am J Hum Genet. 90:331-339.

Porter, M.Y., K. Xie, E. Pozharski, M.R. Koelle, and K.A. Martemyanov. 2010. A conserved protein interaction interface on the type $5 \mathrm{G}$ protein beta subunit controls proteolytic stability and activity of $R 7$ family regulator of $G$ protein signaling proteins. J Biol Chem. 285:41100-41112.

Ross, E.M., and T.M. Wilkie. 2000. GTPase-activating proteins for heterotrimeric G proteins: Regulators of $G$ protein signaling (RGS) and RGS-like proteins. Annu Rev Biochem. 69:795-827.

Sandiford, S.L., Q. Wang, K. Levay, P. Buchwald, and V.Z. Slepak. 2010. Molecular organization of the complex between the muscarinic $M 3$ receptor and the regulator of G protein signaling, Gbeta(5)-RGS7. Biochemistry. 49:4998-5006.

Slepak, V.Z. 2009. Chapter 6 Structure, Function, and Localization of Gbeta5-RGS Complexes. Prog Mol Biol Transl Sci. 86C:157-203.

Zhang, J., B.G. Jeffrey, C.W. Morgans, N.S. Burke, T.L. Haley, R.M. Duvoisin, and R.L. Brown. 2010. RGS7 and -11 complexes accelerate the ON-bipolar cell light response. Invest Ophthalmol Vis Sci. 51:1121-1129.

Zhang, J.H., V.A. Barr, Y.Y. Mo, A.M. Rojkova, S.H. Liu, and W.F. Simonds. 2001. Nuclear localization of $G$ protein b5 and regulator of $G$ protein signaling 7 in neurons and brain. J Biol Chem. 276:10284-10289. 


\section{Appendix III: GPR179 is required for high sensitivity of the mGluR6 signaling cascade in depolarizing bipolar cells}

Manuscript submitted to The Journal of Neuroscience September 20, 2013

Thomas A. Ray ${ }^{1 *}$; Kathryn M. Heath ${ }^{2 *}$; Nazarul Hasan ${ }^{1}$; Ivy S. Samuels ${ }^{3}$; Kirill A. Martemyanov ${ }^{4}$; Neal S. Peachey ${ }^{3,5,6}$; Maureen A. McCall ${ }^{2,7,8,9}$, Ronald G. Gregg ${ }^{1,7,9}$

${ }^{1}$ Department of Biochemistry and Molecular Biology, University of Louisville, Louisville, KY 40202

${ }^{2}$ Department of Psychological and Brain Sciences, University of Louisville, Louisville, KY 40292

${ }^{3}$ Louis Stokes Cleveland VA Medical Center, Cleveland, OH 44106

${ }^{4}$ Department of Neuroscience, The Scripps Research Institute, Jupiter, FL 33458

${ }^{5}$ Cole Eye Institute, Cleveland Clinic, Cleveland, OH 44195

${ }^{6}$ Department of Ophthalmology, Cleveland Clinic Lerner College of Medicine of Case Western Reserve University, Cleveland, $\mathrm{OH} 44195$

${ }^{7}$ Department of Ophthalmology and Visual Sciences, University of Louisville, Louisville, KY 40202 
${ }^{8}$ Anatomical Sciences and Neurobiology, University of Louisville, Louisville, KY 40202

${ }^{9}$ Correspondence to: ron.gregg@louisville.edu or mo.mccall@louisville.edu

${ }^{*}$ contributed equally to this work

Conflict of Interest: The authors declare no competing financial interests

Commercial Interest: No

Correspondence to:

Ronald G. Gregg

Department of Biochemistry \& Molecular Biology

University of Louisville

319 Abraham Flexner Way

Louisville, KY 40202

Phone: (502)-852-6592, FAX: (502) 852-6222, email: ron.gregg@louisville.edu

Acknowledgements: This work was supported by funding from the National Institutes of Health (R01 EY12354 (RGG); R01 014701 (MAM); R21 EY21852 (NSP, RGG); EY018139 (KAM)), the Foundation Fighting Blindness, the Department of Veterans Affairs and unrestricted grants from Research to Prevent Blindness to the University of Louisville and the Cleveland Clinic Lerner College of Medicine of Case Western Reserve University.

\section{ABSTRACT}

The two visual parallel pathways are initiated at the first retinal synapse by signaling between the photoreceptors and two types of bipolar cells. For normal function, ON or depolarizing bipolar cells (DBCs) require the G protein-coupled receptor, mGluR6, its $G$ protein-coupled cascade and the transient receptor potential melastatin 1 (TRPM1) cation channel. Another seven transmembrane protein, GPR179, also is required for DBC function and recruits the regulators of G-protein signaling (RGS) proteins, RGS7 and RGS11, to the dendritic tips of the DBCs. Here we show that GPR179 and 
RGS7/RGS11 play different roles in modulation of the mGluR6 mediated gating of TRPM1. We show that expression of several mGluR6 cascade components including, mGluR6, TRPM1 and nyctalopin are independent of GPR179 expression and GPR179 interacts with TRPM1. In Gpr179 ${ }^{\text {nob5 }}$ mice a small dark-adapted electroretinogram bwave is present that can be enhanced with long flashes, indicating some rod DBC function. Patch clamp recordings demonstrate that Gpr179 ${ }^{\text {nob5 }}$ and $R G S 7^{-/ /} / R G S 11^{-/-}$rod DBCs retain mGluR6-mediated gating of TRPM1 when strong stimuli are used. In contrast, the two mutants differ in that direct gating of TRPM1 by capsaicin in RGS7 ${ }^{-/}$ $/ R G S 11^{-/}$and WT rod DBCs is similar, but severely compromised in Gpr179 nob5 rod DBCs. Noise and standing current analyses indicate that TRPM1 channels in Gpr179 rod DBCs have a lower open probability than in WT, presumably from G protein suppression. We propose that the GPR179 not only localizes RGS7 and RGS11 to the dendritic tips of DBCs, but alters the "state" of the TRPM1 channel by direct interactions.

\section{INTRODUCTION}

Visual processing in the mammalian retina is initiated through two parallel vertical pathways that are established at the synapse between photoreceptors and bipolar cells (BCs). In the dark, photoreceptors constantly release glutamate and in response to a light increment they hyperpolarize, decreasing glutamate release. Two general classes of BCs form synapses with cone photoreceptor terminals and are defined by their response to a light increment. Hyperpolarizing $\mathrm{BCs}$ (HBCs) signal via ionotropic glutamate receptors of the AMPA/Kainate type (Kaneko and Saito, 1983; Saito and Kaneko, 1983; Slaughter and Miller, 1983) and DBCs signal via the metabotropic glutamate receptor 6 (mGluR6) (Masu et al., 1995). A single class of DBC, the rod DBC is postsynaptic to rod photoreceptors (Boycott et al., 1969). As a result defects at this synapse lead to complete congenital stationary night blindness (cCSNB). This defect is 
detected by the absence of the b-wave in the electroretinogram (ERG) in both humans and animal models (Miyake et al., 1986; McCall and Gregg, 2008).

The rod light response is initiated when a decrease in glutamate occupancy of mGluR6 signals the opening of the TRPM1 channel (Audo et al., 2009; Li et al., 2009; Morgans et al., 2009; Shen et al., 2009; Koike et al., 2010b; Morgans et al., 2010; Peachey et al., 2012a). This cascade also includes proteins of known function: the heterotrimeric $\mathrm{G}$ proteins $\mathrm{G \alpha}_{\mathrm{o}}$ and G $\beta 3$ (Dhingra et al., 2000; Dhingra et al., 2002; Koike et al., 2010b; Dhingra et al., 2012), and the regulators of G proteins G $\beta 5$, RGS7 and RGS11 (Morgans et al., 2007; Jeffrey et al., 2010; Cao et al., 2012). Other known components with unresolved function include nyctalopin, GPR179 and LRIT3 (Gregg et al., 2003; Audo et al., 2012; Peachey et al., 2012b; Zeitz et al., 2013).

GPR179 is a seven transmembrane receptor that interacts with RGS7 and RGS11 and is critical for proper localization of these proteins to the DBC dendritic tips (Orlandi et al., 2012). GPR179 expression is required for normal mGluR6 signaling (Peachey et al., 2012b) and mutations in Gpr179 in mouse and human patients cause a no b-wave ERG phenotype (Audo et al., 2012; Peachey et al., 2012b). To gain further insight into the role of GPR179 in the rod DBC light response we studied Gpr179 ${ }^{\text {nob5 }}$ and

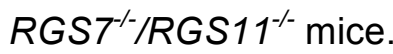

We show that expression of both mGluR6 and TRPM1 are independent of GPR179 expression and that GPR179 and TRPM1 proteins interact. Functional analysis using ERG and whole cell patch clamp recording of rod DBCs confirm that TRPM1 is present in both $G p r 179^{n o b 5}$ and $R G S 7^{-/} / R G S 11^{-/}$rod DBCs, although the sensitivity of the cascade is significantly lower in each compared to WT. However, the two mutants differ in that direct gating of TRPM1 by capsaicin is similar in WT and RGS7//RGS $11^{-/-}$ rod DBCs and severely compromised in Gpr179 ${ }^{\text {nob5 }}$ rod DBCs. Taken together, our results suggest that the GPR179/RGS7/RGS11 complex sets the sensitivity of the 
mGluR6 signaling cascade and that the GPR179/TRPM1 interaction sets the sensitivity of gating of the TRPM1 channel to light stimuli.

\section{Materials and Methods}

\section{Animals}

All procedures were performed in accordance with the Society for Neuroscience policies on the use of animals in research and each local Institutional Animal Care Use Committees. Descriptions of all mice used have been published previously (Masu et al., 1995; Pardue et al., 1998; Pearring et al., 2011; Cao et al., 2012; Peachey et al., 2012b) and every line was either generated on a C57BL/6J background or backcrossed onto this background for at least 6 generations. All mice were housed in local AALAC approved facilities under a $12 \mathrm{~h} / 12 \mathrm{~h}$ light/dark cycle. $\operatorname{Trpm} 1^{-/-}\left(\operatorname{Trpm} 1^{\operatorname{tm} 1 \text { Lex }}\right)$ mice can be obtained from the European Mouse Mutant Archive (emmanet.org) and were originally generated by Lexicon Genetics.

\section{Antibodies}

In experiments to examine the pattern of protein expression in the outer plexiform layer (OPL), the following primary antibodies (and their concentrations) were used: sheep anti-GPR179 (peptide KVQEETPGEDLDRPVLQKR), 1:2,000 (Peachey et al., 2012b); mouse monoclonal anti-ctbp2/Ribeye (BD Bioscience), 1:1,000; guinea pig antimGluR6 1:1,000 (Koike et al., 2010b); sheep anti-TRPM1 1:1,000 (Cao et al., 2011); rabbit anti-GFP (MBL), 1:800 and Rhodamine Peanut Aglutinin (PNA) conjugate 566 (Vector Labs), 1:1,000. Secondary antibodies (Invitrogen, 1:1,000) appropriate to each primary antibody included: donkey anti-sheep Alexa-488, donkey anti-rabbit Alexa-680, donkey anti-rabbit Alexa-546, donkey anti-mouse Alexa-647 and donkey anti-guinea pig Cy3 (Millipore, 1:1,000). In lieu of an antibody specific to nyctalopin, we used Tg(Gabrr1-

YFP/nyx $)^{R g g 1}$ transgenic mice that express a yellow fluorescent protein (YFP) tagged nyctalopin (Gregg et al., 2007). They are labeled WT in the figures. 


\section{Immunohistochemistry}

Mice were anesthetized, their eyes were enucleated and the lens was removed. Eyecups were washed in phosphate buffered saline (PBS) then fixed for 30 minutes in 4\% paraformaldehyde PBS solution ( $\mathrm{pH} 7.4)$. Eyecups were washed 3 times in PBS then cryoprotected in increasing concentrations of sucrose in PBS $(10 \%, 15 \%$ for $1 \mathrm{~h}$ each and $20 \%$ overnight). Eyecups were embedded in $2: 1$ OCT/ $20 \%$ sucrose PBS solution frozen in a liquid nitrogen cooled bath of isopentane. Eyecups were sectioned (18 $\mu \mathrm{m})$ using a Leica 1850 cryostat, mounted on glass slides and stored at $-70^{\circ} \mathrm{C}$. Sections were warmed to $37^{\circ} \mathrm{C}$ and washed with PBS and PBS containing $0.05 \%$ Triton $X-100(P B X)$ for 5 min each, then blocked in PBX 5\% normal donkey serum blocking solution for $1 \mathrm{~h}$. Sections were incubated overnight at room temperature in the presence of the primary antibody diluted in blocking solution, then washed 3 times for 10 min each with PBX followed by incubation in secondary antibody in PBX for $1 \mathrm{~h}$ at room temperature. Sections were washed for $10 \mathrm{~min}$ in PBX twice and in PBS once and cover-slipped using Immu-Mount (Thermo Scientific). Slides were imaged using an Olympus FV1000 confocal microscope. Images were universally adjusted for brightness using Photoshop.

\section{Mass Spectrometry}

Retinas were isolated from WT mice and homogenized in lysis buffer ( $1 \%$ Nonidet P-40, 2 mM EDTA, 20 mM HEPES pH 7.4, supplemented with protease inhibitors cocktail) by rotating at $4^{\circ} \mathrm{C}$ for $45 \mathrm{~min}$. Samples were centrifuged at $17,000 \mathrm{~g}$ for 20 min to remove the debris and supernatant was precleaned with Dynabeads (Invitrogen) for $1 \mathrm{~h}$ at $4^{\circ} \mathrm{C}$. Samples were incubated with TRPM1 or GPR179 antibody overnight at $4^{\circ} \mathrm{C}$. Lysates and antibody complexes were incubated with Dynabeads for $1.5 \mathrm{~h}$ at $4^{\circ} \mathrm{C}$. Protein complexes were eluted with Nupage LDS sample buffer (Invitrogen) and electrophoresed on Nupage gel (Invitrogen) until the highest molecular weight 
standard (260 kDa) had moved $\sim 5 \mathrm{~mm}$ into the gel. Electrophoresed gel pieces were cut from the top of the gel and an in-gel tryptic digestion was performed as described previously (Rood et al., 2010).

The resulting peptide mixture was resolved by liquid chromatography (LC) using an EASY n-LC (Thermo Scientific) UHPLC system with buffer $A=2 \%$ v/v acetonitrile / $0.1 \% \mathrm{v} / \mathrm{v}$ formic acid and buffer $\mathrm{B}=80 \% \mathrm{v} / \mathrm{v}$ acetonitrile $/ 0.1 \% \mathrm{v} / \mathrm{v}$ formic acid as mobile phases. The mass spectrometry data from LC elutes was collected using an Orbitrap Elite - ETD mass spectrometer (Thermo Scientific). A decision tree was used to determine whether CID or ETD activation was used. Proteome Discoverer v1.3.0.330 was used to analyze the data collected by the mass spectrometer. Scaffold v3.6.5 was used to calculate the false discovery rate using the Peptide and Protein Prophet algorithms.

\section{Cell culture, transfection and immunoblotting:}

HEK293T (Human Embryonic Kidney) cells were cultured in high glucose DMEM supplemented with $10 \%$ fetal bovine serum, $2 \mathrm{mM} \mathrm{L-glutamine,} 50 \mathrm{IU} / \mathrm{ml}$ penicillin and $50 \mu \mathrm{g} / \mathrm{ml}$ streptomycin. One day prior to transfection, cells were seeded on $60 \mathrm{~mm}$ culture dishes. Gpr179 and Trpm1 expression plasmids were transfected into HEK293T cells using JetPrime reagent (Polyplus-transfection) or Lipofactamine 2000 (Invitrogen) according to the manufacturer's instructions. After $24-48 \mathrm{~h}$ of transfection, cells were harvested in NP-40 lysis buffer (50 mM Tris, 150 mM NaCl, 2 mM EDTA, 1\% Nonidet P40, $\mathrm{pH}$ 8.0, supplemented with protease inhibitors cocktail (Sigma-Aldrich) by rotating for $45 \mathrm{~min}$ at $4^{\circ} \mathrm{C}$ and centrifuged at $17,000 \mathrm{~g}$ for $15 \mathrm{~min}$ at $4^{\circ} \mathrm{C}$. Protein content was quantified by Bradford reagent (BioRad). Protein lysates were analyzed on $4-12 \%$ Nupage gels (Invitrogen) or 6\% SDS-PAGE gels, transferred to PVDF membranes and immunoblotted using HRP-conjugated secondary antibodies and ECL West Pico detection system (Thermo Scientific). 


\section{Co-immunoprecipitation}

Dissected mouse retinas were homogenized in lysis buffer (1\% Nonidet P-40, 2 mM EDTA, 20 mM HEPES pH 7.4, supplemented with protease inhibitors cocktail) by rotating at $4^{\circ} \mathrm{C}$ for $45 \mathrm{~min}$. Homogenates were cleared by centrifugation at $17,000 \mathrm{~g}$ for $20 \mathrm{~min}$ at $4^{\circ} \mathrm{C}$. For co-immunoprecipitation, retinal lysates or transfected HEK cells lysates were precleared by incubating with $12 \mu$ l Dynabeads protein G (Invitrogen) at $4^{\circ} \mathrm{C}$ for $1 \mathrm{~h}$. Precleared lysates were incubated with 2-5 $\mu \mathrm{g}$ of anti-GPR179 or anti-Flag antibodies overnight at $4^{\circ} \mathrm{C}$ on an orbital rocker. $45 \mu \mathrm{l}$ of Dynabeads protein $\mathrm{G}$ were added and incubated for $1-2 \mathrm{~h}$ at $4^{\circ} \mathrm{C}$. Dynabeads were collected and washed 4 times with Tris-buffered saline containing $0.3 \%$ Tween-20. Protein complexes were eluted with $40 \mu \mathrm{l}$ of $4 \mathrm{X}$ LDS loading buffer by incubation at $70^{\circ} \mathrm{C}$ for $10 \mathrm{~min}$, separated by SDSPAGE and analyzed by immunoblotting.

\section{Electroretinography}

Mice were tested in two recording protocols in which either a strobe flash or variable duration stimuli were used to evoke ERG responses. For both, animals were dark-adapted overnight and anesthetized with ketamine $(80 \mathrm{mg} / \mathrm{kg})$ and xylazine (16 $\mathrm{mg} / \mathrm{kg}$ ). Eyedrops were used to dilate the pupil (1\% tropicamide, $2.5 \%$ phenylephrine $\mathrm{HCl})$ and to anesthetize the corneal surface (1\% proparacaine $\mathrm{HCl})$. ERGs were recorded using a stainless steel (strobe flash) or gold (variable duration) electrodes contacting the corneal surface wetted with $1 \%$ methylcellulose. Platinum needle electrodes in the cheek and tail serve as reference and ground, respectively. Strobe flash ERGs were recorded using an LKC UTAS E-3000 signal averaging system to stimuli that ranged in luminance from -3.6 to $1.4 \mathrm{log} \mathrm{cd} \mathrm{sec} / \mathrm{m}^{2}$. ERGs were also recorded using a Diagnosys Espion system with a $-1.2 \mathrm{log} \mathrm{cd} / \mathrm{m}^{2}$ stimulus that ranged in duration from $10-1000$ msec.

\section{Retinal Slice Preparation and Whole Cell Patch Clamp Recording}


Mice were anesthetized with an intraperitoneal injection of Ringer's solution containing ketamine/xylazine (127/12 mg/kg, respectively) and sacrificed by cervical dislocation. The eyes were enucleated and the retinas removed and placed in fresh Ames solution (Sigma-Aldrich). The retina was adhered to nitrocellulose paper (Millipore) and then sliced perpendicular to the retinal layers using a tissue slicer. The slices were then placed in a recording chamber. Recording electrodes were pulled from borosilicate glass (FHC, Inc., Bowdoin, ME) on a P-97 Flaming/Brown Micropipette Puller (Sutter Instruments Co., Novato, CA). Electrode resistance measured between 6-9 M $\Omega$. Glass electrodes were filled with intracellular solution that contained Cs-gluconate solution (20 mM CsCl, 107 mM CsOH, 107 mM D-Gluconic Acid, 10 mM NaHEPES, 10 mM BAPTA, $4 \mathrm{mM}$ ATP, and $1 \mathrm{mM}$ GTP). The intracellular solution contained $1 \%$ sulforhodamine to visualize the cell and classify its morphology (Ghosh et al., 2004). Rod DBC somas were targeted for whole cell patch clamp recording. A 2-4 G $\Omega$ seal was created on the cell body and cells with an input resistance $\sim 1 \mathrm{G} \Omega$ and access resistance $<25 \mathrm{M} \Omega$ were used for recording. The recording chamber was maintained at $34-35^{\circ} \mathrm{C}$.

To block inhibitory inputs, Ames solution was supplemented with: $1 \mu \mathrm{M}$ strychnine, $100 \mu \mathrm{M}$ picrotoxin and $50 \mu \mathrm{M}$ 6-tetrahydropyridin-4-yl methylphosphinic acid (TPMPA). Four $\mu \mathrm{M}$ L-AP4 was added to the bath solution to saturate mGluR6 receptors. The mGluR6 receptor antagonist a-cyclopropyl-4-phosphonophenylglycine (CPPG) was dissolved in Ames medium to a working concentration of $0.6 \mathrm{mM}$ or $3 \mathrm{mM}$. CPPG was applied by pressure application using a Picospritzer II (Parker Instrumentation, Cleveland, $\mathrm{OH}$ ) onto the rod $\mathrm{DBC}$ dendritic tips in the outer plexiform layer (OPL). In separate experiments, capsaicin $(10 \mu \mathrm{M})$, a TRPM1 agonist, was puffed onto the rod DBC dendrites to gate the opening of the TRPM1 channel. Reagents were purchased from Sigma-Aldrich, except for L-AP4, CPPG, and capsaicin, which were purchased from Tocris Bioscience. 


\section{Voltage Clamp Protocols}

Rod DBC responses were recorded via a Multiclamp 700A amplifier with a Digidata 1440A digitizer (MDS Analytical Technologies, Union City, CA) and filtered at $2.4 \mathrm{kHz}$ with a four-pole Bessel low pass filter, sampled at $10 \mathrm{kHz}$. Clampex 10.2 software (MDS Analytical Technologies, Sunnyvale, CA) was used to generate command outputs and acquire and analyze analog whole cell current. Rod DBCs were voltage clamped at $+50 \mathrm{mV}$ (Nawy, 2004; Shen et al., 2009). CPPG was puffed at rod DBC dendrites for either $200 \mathrm{msec}$ or $1 \mathrm{sec}$. For capsaicin experiments $200 \mathrm{msec}$ and 1 sec puffs were applied. Three to five responses were recorded from each cell and then averaged. Variance and standing current were measured across the first $1.5 \mathrm{sec}$ of the recording for each rod $\mathrm{DBC}$. Offline analyses of data were performed using Clampfit 10.2. Prism 5.04 software (Graphpad Software, Inc., La Jolla, CA) was used to perform two-way repeated measures ANOVAs, two-way ANOVAs, one-way ANOVAs, or t-tests as suited for the necessary comparison. Statistical significance $=P<0.05$.

\section{RESULTS}

The localization of mGluR6, TRPM1 and NYX to the DBC dendritic tips is independent of GPR179 expression

Since GPR179 is critical to localization of the RGS7 and RGS11 (Orlandi et al., 2012), we examined if it plays a similar role for other proteins in the DBC cascade. We compared expression and localization of mGluR6, the receptor that initiates signaling and TRPM1 the channel that is ultimately gated by the cascade in the outer retina of WT and Gpr179 ${ }^{\text {nob5 }}$ mice. We also examined the expression pattern of nyctalopin (EYFPNyx), a protein critical to expression/localization of TRPM1 (Pearring et al., 2011). Fig. 1 shows representative transverse retinal section reacted with antibodies to label these three proteins in WT (A) and Gpr179 nob5 retina. The expression pattern is indistinguishable between WT and Gpr179 nob5. The merged images in triple labeled 
retinal sections show that TRPM1, mGluR6 and nyctalopin expression overlaps on the dendritic tips of the DBCs. 
A
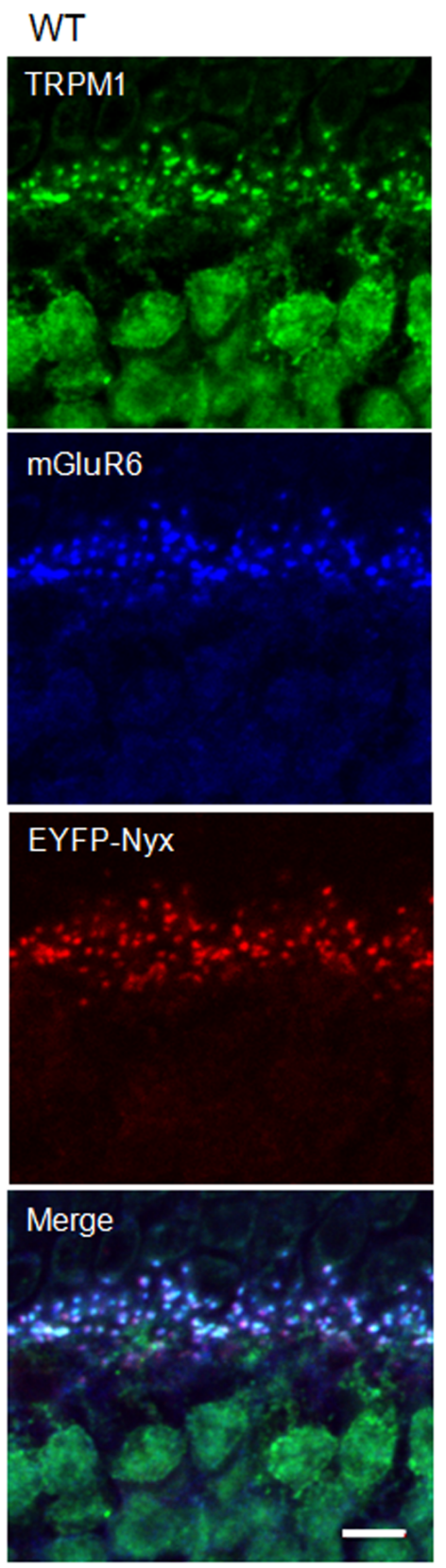

B
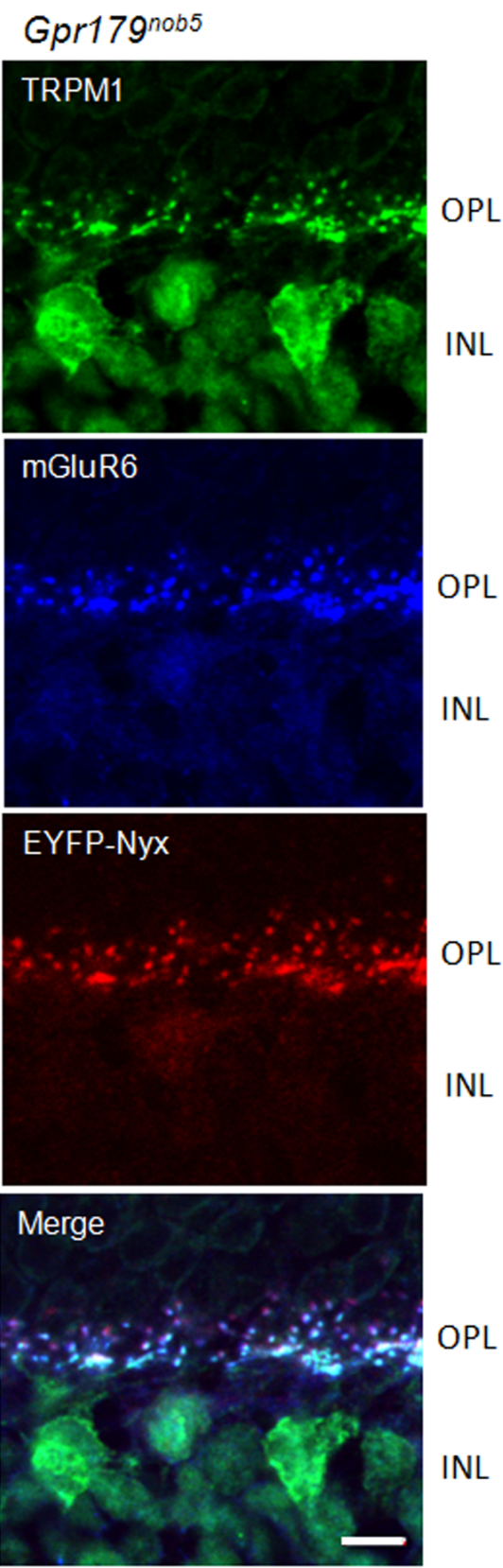

Figure 1. mGluR6, TRPM1 and NYX expression is independent of GPR179 expression. Representative confocal images of cross sections of the OPL and INL of WT $(A)$ and $G p r 179^{n o b 5}(B)$ retinas reacted with antibodies to TRPM1 (green), mGluR6 (blue) and EYFP-Nyc (red). The merged images (bottom) show that in both WT and in Gpr179 $^{\text {nob5 }}$ retinas expression patterns are similar and that TRPM1, mGluR6 and nyctalopin expression co-localize at the dendritic tips of DBCs. Scale bars, $5 \mu \mathrm{m}$. 
To determine if GPR179 expression depends on expression of mGluR6, Nyctalopin and TRPM1, we reacted retinal sections from WT and knockout or mutant mice: $\mathrm{Grm6}^{--}$(Grm6 encodes mGluR6), Nyx ${ }^{\text {nob }}$ and $\mathrm{Trpm}^{-/-}$with an antibody to GPR179 (Peachey et al., 2012b). We co-labeled these sections with the lectin, PNA, to mark the cone pedicles in the OPL. Fig. 2 shows that the pattern of GPR179 expression is indistinguishable in representative sections from all four mouse strains. As expected, GPR179 expression is absent in the negative control, Gpr179 ${ }^{\text {nob5 }}$ retina (Peachey et al., 2012b). 


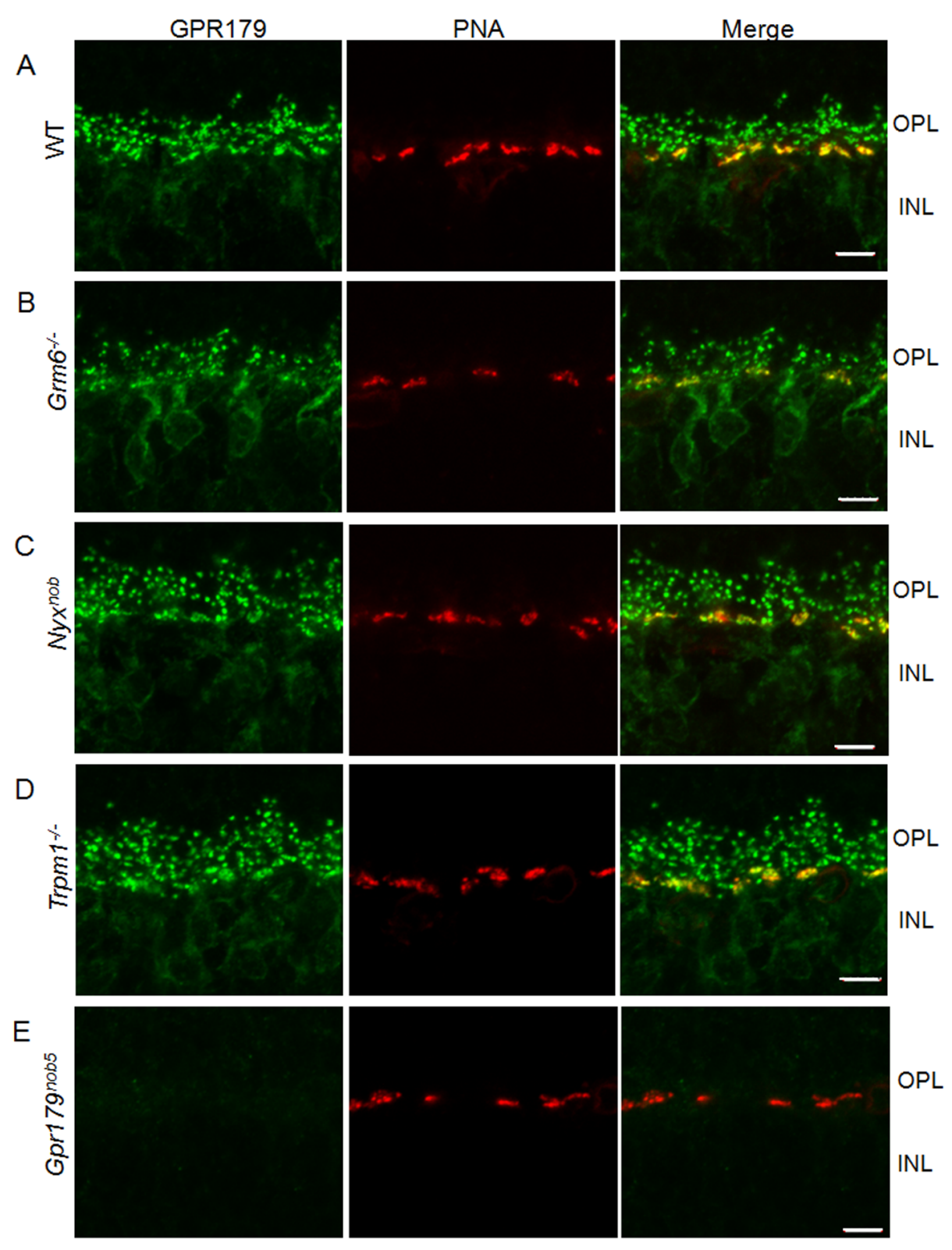

Figure 2. GPR179 expression at the dendritic tips of the DBCs is independent of mGluR6, TRPM1 and nyctalopin expression. Representative confocal images of cross sections of the OPL and INL of a series of WT and mutant mouse retinas reacted with an antibody to GPR179 (green) and the cone pedicle marker, PNA (red). (A) In WT retinas expression of GPR179 colocalizes with PNA (yellow puncta in merged image). (B-D) GPR179 expression also is localized at the dendritic tips of DBCs even in the absence of expression of: mGluR6 (B), nyctalopin (C) and TRPM1 (D). As shown here (E) and previously (Peachey et al., 2012b) GPR179 expression is absent from Gpr179 ${ }^{n o b 5}$ mouse retina. Green puncta in the merged images represent GPR179 expression at the dendritic tips of rod DBCs. Scale bars, $5 \mu \mathrm{m}$. 


\section{Independence of localization of GPR179, mGluR6, TRPM1, and nyctalopin}

Although not evident in Figs 1 and 2, we observed what might be subtle differences in the level of GPR179 expression across these mutants. We used western blotting from retinal lysates of each to examine and to quantify expression levels of GPR179 relative to WT (Fig. 3A,B). As expected, the negative control, Gpr179 nob5 mutant retina showed no expression. In $\mathrm{Grm}^{-/}$and $\operatorname{Trpm}^{1 /-}$ mutant retina, expression levels of GPR179 were reduced, by $\sim 50 \%$ and $\sim 20 \%$, respectively. In addition, we examined and quantified TRPM1 expression levels in $\mathrm{Gpr}_{179^{n o b 5}}$ and $\mathrm{Grm6} 6^{-/}$retinas (Fig. 3C,D). Again, the negative control shows no expression in the TPRM1 ${ }^{-/}$. In Gpr179 $19^{\text {nob5 }}$ retinas, TRPM1 expression is decreased by $\sim 30 \%$, but is similar to WT in $\mathrm{Grm6}^{-/}$retina. Others have reported that TRPM1 is mislocalized in Grm6 mutant retina (Morgans et al., 2009; van Genderen et al., 2009; Cao et al., 2011; Pearring et al., 2011; Peachey et al., 2012a; Xu et al., 2012) and it has been shown that TRPM1 expression is decreased $\sim 30 \%$ in the $G r m 6^{n o b 3}$ mutant (Cao et al., 2011). The discrepancy between our results and previously published results could be due to animal age or variation in the Grm6 mutant (Maddox et al., 2008; McCall and Gregg, 2008). In summary, our data show that GPR179 is localized correctly in the OPL independent of mGluR6, TRPM1, and nyctalopin and that the localization of these proteins is independent of GPR179, although expression levels may change.

\section{GPR179 and TRPM1 are part of the same protein complex}

We have previously shown a physical interaction between TRPM1 and nyctalopin (Cao et al., 2011; Pearring et al., 2011) and between GPR179 and RGS7 and RGS11 (Orlandi et al., 2012). Using an affinity purification by immunoprecipitation (IP) approach, we examined GPR179 interactions with TRPM1. Using antibodies to GPR179, we immunoprecipitated protein complexes from WT mouse retinal lysates. The identity of co-immunoprecipitated proteins was characterized using mass spectroscopy. IP with 
antibodies to GPR179 (a positive control) yielded 121 peptides identified as GPR179 and giving a coverage of $55 \%$. Two peptides were identified as TRPM1. IP with antibodies to TRPM1 yielded 48 peptides matching TRPM1 (57\% coverage). Twelve peptides were identified as GPR179 (9\% coverage). As a negative control for the specificity of the assay, we used an identical IP approach with a non-specific IgG. None of the peptides in the resulting data set matched either GPR179 or TRPM1. Additional negative controls included IPs from GPR179 ${ }^{\text {nob5 }}$ retinas with GPR179 antibodies and IPs from $\mathrm{Trpm}^{-/}$retinas with TRPM1 antibodies. In these latter two controls, neither TRPM1 nor GPR179 were identified in the resulting data sets. Combined these data indicate our IPs were specific and that GPR179 and TRPM1 are part of the same protein complex.

To test the validity of the mass spectrometry results, and to determine whether GPR179 and TRPM1 interact directly, we used IP from HEK293T cells co-transfected with Gpr179 and FLAG-Trpm1 expression vectors (Pearring et al., 2011) and from WT mouse retinas (Fig. 3 E,F). Western blot analyses of singly (Fig. 3E, lanes 1,2) and doubly (Fig. 3E, Lane 3) transfected HEK293T cells show that both proteins were expressed as expected. IP with the FLAG antibody (Fig. 3E, lanes 4-6) shows on the singly transfected cells (Fig. 3E, lanes 4,5 ) that it precipitates FLAG-TRPM1 only, as expected. IP from the double transfected cells shows that the FLAG antibody co-IPs GPR179 and FLAG-TRPM1 (Fig. 3E, lane 6), indicating that the two proteins interact. To control for the possibility that this interaction occurs during the lysis procedure, we transfected GPR179 and FLAG-TRPM1 expressing into different HEK293T cell cultures, lysed the cells and mixed the lysates. IP with FLAG antibodies and subsequent western blotting for GPR179 showed that GPR179 is absent from the immunoprecipitates (data not shown), supporting the conclusion that GPR179 and TRPM1 when expressed in HEK293T cells interact directly. 
When we repeated the IP experiments using retinal lysates (Fig. 3F), both GPR179 and TRPM1 were present in WT as expected (Fig. 3F, lane 1), and GPR179 was absent in Gpr179 ${ }^{\text {nob5 }}$ retinal lysate (Fig. 3F, lane2). IP from WT retinal lysates with an antibody to GPR179 and subsequent western blotting of the precipitates shows that TRPM1 is co-immunoprecipitated, along with GPR179 (Fig. 3F, lanes 3,5). As controls, we repeated the IP experiments using lysates from Gpr179 ${ }^{\text {nob5 }}$ (Fig. 3F, lane 4) and Trpm1 $^{-/}$(Fig. 3F, lanes 6) retinas. Both GRP179 and TRPM1 were absent in precipitates from the Gpr179 nob5 retinas (Fig. 3F, lane 4) and only GPR179 was present in precipitates from Trpm $^{-/}$retinas (Fig. 3F, lane6). Combined the mass spectrometry and IP studies support the conclusion that GPR179 and TRPM1 are part of the same synaptic complex and interact directly. 

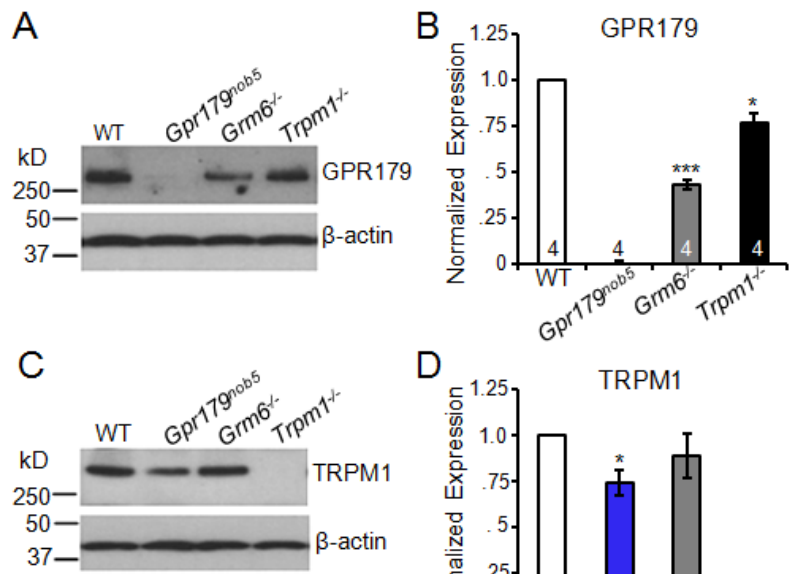

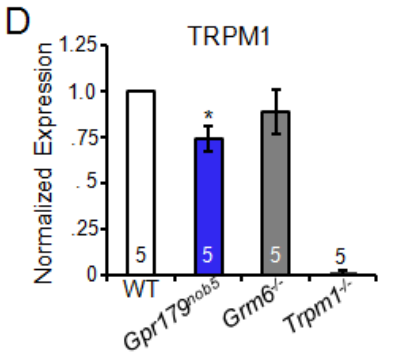

E

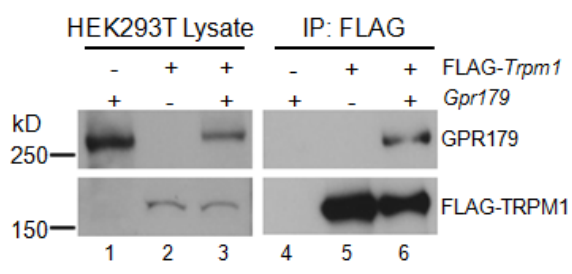

$\mathrm{F}$

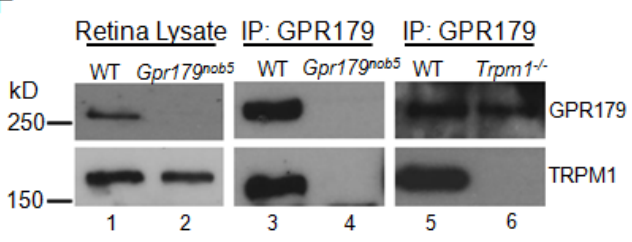

Figure 3. GPR179 interacts with TRPM1. Western blots of total retinal lysates probed with antibodies to (A) GPR179 and (C) TRPM1. Each blot was reprobed with antibodies to $\beta$-actin to determine total protein and for use as an internal standard. Band intensities were analyzed and quantified with NIH ImageJ software and normalized to the $\beta$-actin expression level in the same sample. The histograms ( $B, D)$ plot the mean expression from four experiments on independent samples. (B) GPR179 expression was lower in $\mathrm{Grrm}^{-/-}$and Trpm1 ${ }^{-/-}$ retinas compared to WT. (D) TRPM1 expression was similar to WT in $\mathrm{Grm}^{-/-}$and in GRP179 ${ }^{\text {nob5 }}$ retinas. Errors bars represent standard error ${ }^{*} P<0.05,{ }^{* * *} P<0.001$. (E) Western blot of HEK293T cells transfected with plasmids expressing GPR179 (lane 1), FLAG-TRPM1 (lane 2) or both (lane 3) and probed with GPR179 and FLAG antibodies. The presence of a specific expression construct is indicated by "+" above the lane on the blot. Lysates from HEK293T samples (lanes 1-3) were immunoprecipated with antibodies to GPR179 and the precipitates analyzed by western blotting, using antibodies to GPR179 (lanes 4-6, top row) or TRPM1 (lanes 4-6, bottom row). These data show that TRPM1 is co-immunoprecipitated with GPR179 (lane 6). (F) Western blot of retinal lysates from WT (lane 1) and GPR179 $^{\text {nob5 }}$ (lane 2) retinas, probed for presence of GPR179 (top row) or TRPM1 (bottom row). Western blots of proteins co-immunoprecipated with antibodies to GPR179 from WT (lanes 3 and 5), GPR179 ${ }^{\text {nob5 }}$ (lane 4) and $\operatorname{Trpm}^{-/ /}$(lane 6) probed for GPR179 (top row) or TRPM1 (bottom row). Immunoprecipitation with GPR179 antibody from retinal lysates of $\mathrm{Gpr}_{179^{\mathrm{Nob5}}}$ and $\mathrm{Trpm} 1^{-/-}$mice served as controls for nonspecific binding. These data were representative of at least three independent experiments using independent samples. Data show that GPR179 and TRPM1 co-immunoprecipate (lanes 3 and 5). 


\section{Gpr179 $^{\text {nob5 }}$ mice have ERG b-waves absent in other cCSNB mouse models}

Previously we compared the ERG responses of WT and Gpr179 nob5 and showed a significant reduction in the $G p r 179^{n o b 5} \mathrm{~b}$-wave and interpreted this result as a no bwave phenotype (Peachey et al., 2012b). When ERG responses from Gpr179 ${ }^{\text {nob5 }}$ and $\mathrm{Trpm}^{-1-}$ mice were compared to WT (Fig. 4A) their positive polarity b-waves appear to be completely missing. However, upon closer inspection of these mutant responses at two low flash luminances $\left(-3.6,-2.4 \log \mathrm{cd} \mathrm{sec} / \mathrm{m}^{2}\right)$, where the a-wave and slow PIII are small in amplitude, we found a b-wave like response, albeit small $(\sim 15-20 \mu \mathrm{V})$, that was consistently present in Gpr179 $9^{\text {nob5 }}$ but not in $\operatorname{Trpm}^{-/-}$mice (Fig. 4B). When quantified, the b-wave like response was significantly larger in $G p r 179^{n o b 5}$ than $T r p m 1^{-/}$mice (t-test; $P<0.001$; Fig. 4C). 
A

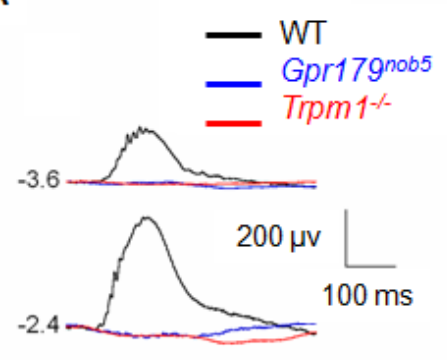

B

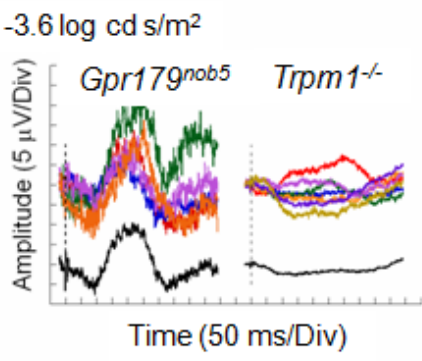

C

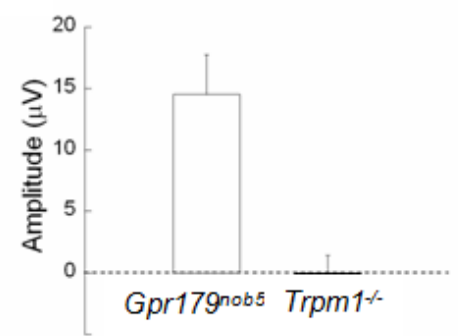

Figure 4. Gpr179 ${ }^{\text {nob5 }}$ rod ERGs have a small b-wave. (A) Representative rod ERGs recorded from WT (black), Gpr179 ${ }^{\text {nob5 }}$ (blue) and $\operatorname{Trpm1}^{-/-}$(red) mouse retinas to strobe flash stimuli presented to the dark-adapted retina. Note that the positive polarity b-wave of the WT ERG is missing in Gpr179 ${ }^{\text {nob5 }}$ and $\operatorname{Trpm} 1^{-/}$mice. Values to the left of the waveforms indicate flash luminance in $\log \mathrm{cd} \mathrm{sec} / \mathrm{m}^{2}$. (B) ERG responses obtained from Gpr179 ${ }^{n o b 5}$ and $\operatorname{Trpm}^{-/}$mice to a $-3.6 \mathrm{log} \mathrm{cd} \mathrm{sec} / \mathrm{m}^{2}$ flash. Colored traces indicate the responses from 5 different $G p r 179^{\text {nob5 }}$ mice (left) and 7 different $\operatorname{Trpm}^{-/-}$mice (right). The offset black trace in each panel is the average of all mice. (C) Average ( \pm sem) b-wave amplitude (difference between pre-stimulus baseline and the largest positive deviation from the baseline recorded between 100 and $300 \mathrm{msec}$ after flash presentation) of responses shown in (B). Note that in GPR179 ${ }^{\text {nob }}$ mice there is a response that is absent in the Trpm1s. mice. 


\section{GPR179 sets the sensitivity of mGluR6 cascade modulation of TRPM1}

Our observations that both mGluR6 and TRPM1 proteins are expressed in the OPL of Gpr179 ${ }^{\text {nob5 }}$ retina (Fig. 1), as well as the presence of a small b-wave in these mice (Fig. 3), suggest that mGluR6 and its cascade still gate the TRPM1 channel, although the sensitivity of the system is significantly reduced. To address this question, we tested several elements of this hypothesis using whole cell patch clamp recordings of rod DBCs in a retinal slice preparation. We used the methods described by Nawy and colleagues (Nawy, 2004; Shen et al., 2009) in which retinal slices were bathed in $4 \mu \mathrm{M}$ L-AP4 to maximally activate the mGluR6 cascade and close TRPM1 channels. Puffs of CPPG, a mGluR6 antagonist, then were used to deactivate the cascade, mimicking the events evoked by light. We held rod DBCs at $+50 \mathrm{mV}$ to minimize run-down and, as a consequence, outward currents are evoked by CPPG puffs.

We examined whether the TRPM1 channel could be gated by the mGluR6 cascade by exogenous application (puffs) of CPPG onto the dendritic terminals of synaptically isolated Gpr $179^{n o b 5}$ rod DBCs. Fig. 5A shows representative responses from WT and Gpr179 ${ }^{\text {nob5 }}$ rod DBCs to $0.6 \mathrm{mM} \mathrm{CPPG}$ puffs $200 \mathrm{msec}$ in duration. In WT rod DBCs there is a robust outward current. The response, while present in Gpr $179^{\text {nob5 }}$ rod DBCs was significantly smaller than WT (Fig. 5B; two-way ANOVA: $P<0.001$ ). Increasing puff duration to $1 \mathrm{sec}$ produced no discernible change in WT responses (twoway ANOVA: $P>0.05$; data not shown), but significantly increased the response in Gpr179 ${ }^{\text {nob5 }}$ rod DBCs (Fig. 5B; two-way ANOVA: $P<0.001$ ). Despite this increased Gpr179 ${ }^{\text {nob5 }}$ response, the amplitude was still significantly smaller than in WT (two-way ANOVA: $P<0.001$; Fig. 5A, B). We attempted to increase stimulation further by increasing the concentration of CPPG to $3 \mathrm{mM}$. At both $200 \mathrm{msec}$ and $1 \mathrm{sec}$ puff duration, WT responses remained similar to those evoked by $0.6 \mathrm{mM}$ CPPG (Fig. 5C,D), indicating that the mGluR6 cascade was maximally activated by $0.6 \mathrm{mM}$ and $200 \mathrm{msec}$ 
puffs. In Gpr179 nob5 rod DBCs, increasing the CPPG concentration to 3mM yielded responses similar in magnitude, regardless of puff duration. They also were similar to the response evoked by $0.6 \mathrm{mM} / 1$ sec CPPG puffs. This suggests that the Gpr $179^{\text {nob5 }}$ rod DBC response can be saturated at the same concentration as WT but requires longer duration puffs (Fig. 5B,D). Regardless of the conditions, the Gpr179 nob5 rod DBC responses never reached the amplitude recorded in WT (Fig. 5C,D; two-way ANOVA: 200 msec: $P<0.001 ; 1$ sec: $P<0.001)$. Together these data suggest that while GPR179 is not required to gate TRPM1, it sets the sensitivity of the mGluR6 cascade and modulation of TPRM1 in rod DBCs. 
A

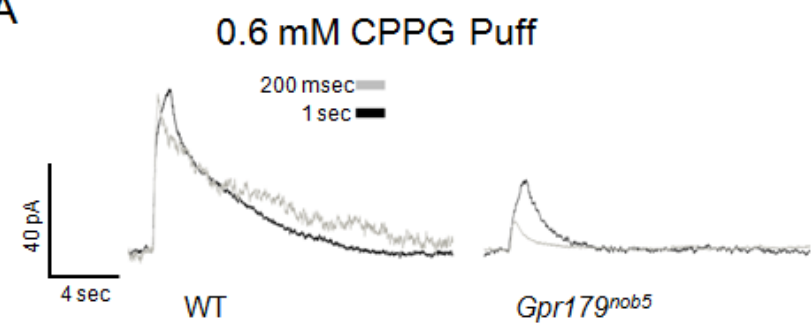

C
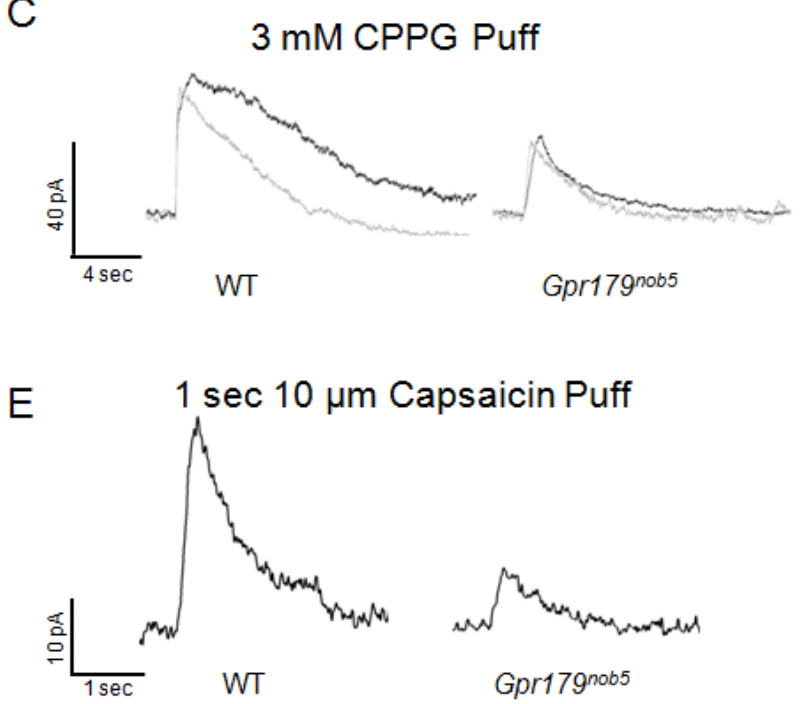

$\mathrm{B}$

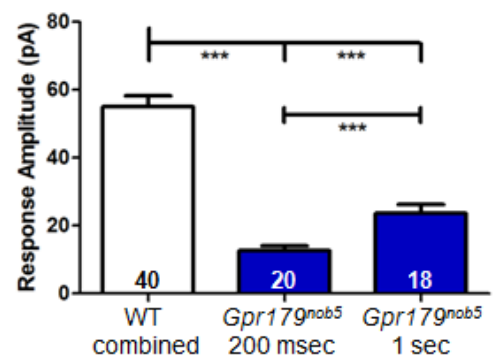

D

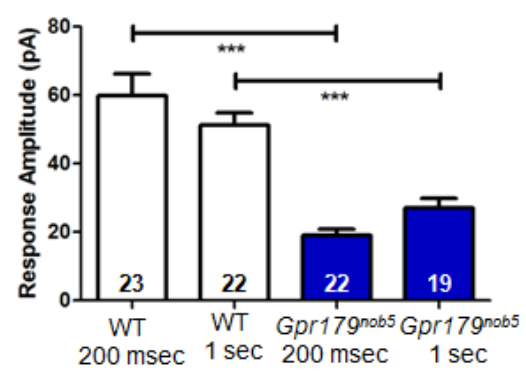

$\mathrm{F}$

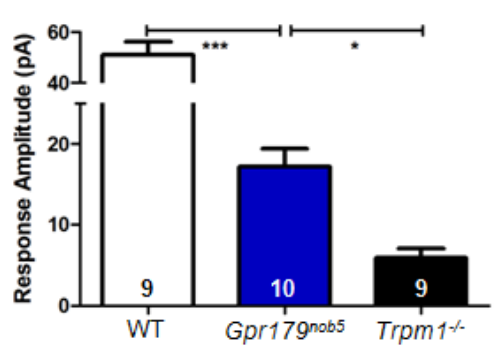

Figure 5. CPPG evokes a small amplitude response in Gpr179 ${ }^{\text {nob5 }}$ rod DBCs, which increases with stimulus intensity. (A) Representative voltage clamp responses of WT and Gpr179 ${ }^{\text {nob5 }}$ rod DBCs evoked by puff application of the mGluR6 antagonist CPPG $(0.6 \mathrm{mM} ; 200 \mathrm{msec}$ or $1 \mathrm{sec})$. (B) Histogram compares the average peak response amplitudes of WT and Gpr179 $170 b 5$ rod DBCs. WT responses to $200 \mathrm{msec}$ and $1 \mathrm{sec}$ puffs did not differ and were combined. Regardless of duration Gpr179 ${ }^{\text {nob5 }}$ response amplitudes were significantly smaller than WT. Gpr179 ${ }^{\text {nob5 }}$ response amplitudes significantly increase when puff duration increased from 200 msec to 1 sec. (C) Representative voltage clamp responses of WT and Gpr179 ${ }^{\text {nob5 }}$ rod DBCs evoked by puff application of $3 \mathrm{mM}$ CPPG for either $200 \mathrm{msec}$ or $1 \mathrm{sec}$. (D) Histogram compares the average peak response amplitudes of WT and Gpr179 ${ }^{\text {nob5 }}$ rod DBCs. WT responses did not increase with increased puff duration (200msec to $1 \mathrm{sec})$. Regardless of duration $G p r 179^{\text {nob5 }}$ response amplitudes were significantly smaller than WT. Increased puff duration did not produce larger response amplitudes in Gpr179 ${ }^{\text {nob5 }}$ rod DBCs suggesting that they are saturated under these conditions. (E) Representative voltage clamp responses of WT and Gpr179 $^{\text {nob5 }}$ rod DBCs evoked by a $1 \mathrm{sec}$ puff of the TRPM1 channel agonist, capsaicin $(10 \mu \mathrm{M})$. (F) Histogram compares the average peak response amplitudes of WT, Gpr179 ${ }^{\text {nob5 }}$ and Trpm1//rod DBCs. Gpr179 ${ }^{\text {nob5 }}$ response amplitudes are significantly larger than $\mathrm{Trpm}^{-/-}$although significantly smaller than WT rod DBCs. The number of rod DBCs in each experimental group is shown within each bar of the histograms. 
Because TRPM1 is expressed in $G p r 179^{\text {nob5 }}$ retina we characterized whether the channel could be gated directly by examining rod DBC responses in WT and Gpr179 ${ }^{\text {nob5 }}$ to the TRPM1 agonist capsaicin. As observed previously (Shen et al., 2009), capsaicin evoked robust responses in WT rod DBCs (Fig. 5E). In contrast, capsaicin evoked responses in Gpr179 ${ }^{\text {nob5 }}$ rod DBCs were significantly smaller than WT. As a control, we examined capsaicin evoked responses in $\mathrm{Trpm}^{-/}$rod DBCs and found they were significantly smaller than present in $G p r 179^{\text {nob5 }}$ rod DBC (Fig. 5F; one-way ANOVA: vs WT: $P<0.001 ;$ vs Trpm1 $\left.1^{-1} P<0.05\right)$. These data are consistent with our immunohistochemical results and suggest that the TRPM1 channel is present in Gpr $179^{\text {nob5 }}$ rod DBCs and can be modulated either by the mGluR6 cascade or directly by capsaicin. Because strong stimuli (both pharmacological and light in the ERG results) are required and the response never reaches the amplitude seen in WT cells we conclude that GPR179 modulates the sensitivity of the cascade. The data also address an issue noted previously, that the rod DBC response to capsaicin is primarily via modulation of TRPM1 channels with a minor contribution from an "off-target" nonTRPM1 channel (Morgans et al., 2009).

In the above recordings, we noted that spontaneous currents in WT appeared noisier than in Gpr179 ${ }^{\text {nob5 }}$ rod DBCs (Fig. 6A), This suggested that the open probability of the TRPM1 channel might differ between WT and Gpr179 ${ }^{\text {nob5 }}$ rod DBCs, as we had noted for $N y x^{n o b}$ rod DBCs (Gregg et al., 2007). To evaluate this question, we measured both the standing outward current and the current variance of rod DBCs $\left(V_{\text {hold }}+50 \mathrm{mV}\right)$. The outward holding currents as well as the variance were significantly larger in WT rod DBCs compared to Gpr179 ${ }^{\text {nob5 }}$ (Fig. 6B,C; one-way ANOVA: holding current: $P<0.001$; variance: $P<0.01)$. As a control we analyzed the holding current and variance in $\mathrm{Trpm}^{-/-}$rod DBCs and found that they were similar to Gpr179 ${ }^{\text {nob5 }}$ cells (Fig. 6; one-way ANOVA: holding current: $P>0.05$; variance: $P>0.05)$. These results support the idea 
that while the TRPM1 channels are correctly expressed and localized, they are in a functionally closed state compared to those in WT rod DBCs. The results continue to support the hypothesis that GPR179 is required for the high sensitivity of the modulation of the TRPM1 channel by the mGluR6 cascade. 

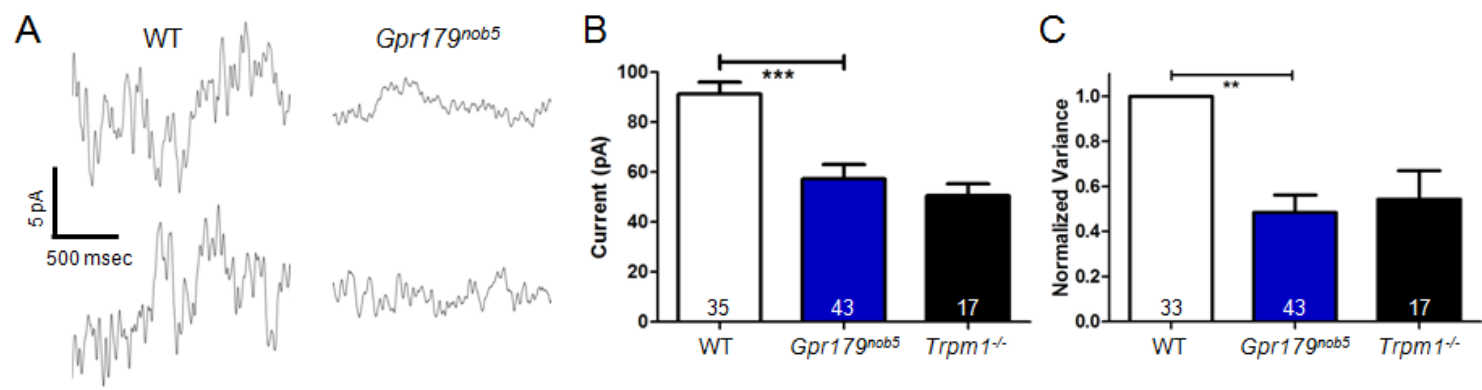

Figure 6. Gpr179 $19^{\text {nob5 }}$ rod DBCs have decreased standing currents and TRPM1 channel open probability. (A) Representative traces of spontaneous currents from two WT and two Gpr179 ${ }^{\text {nob5 }}$ rod DBCs. Rod DBCs were held at $+50 \mathrm{mV}$ and $1.5 \mathrm{sec}$ sections of each recording were analyzed to yield the data in B and C. Histograms show average (B) standing current and (C) current variance for WT, Gpr179 ${ }^{\text {nob5 }}$ and $T_{r p m 1^{-/}}$rod DBCs. Gpr179 ${ }^{\text {nob5 }}$ and $\operatorname{Trpm}^{-/ /}$have similar standing currents (C) and current variance (D) and both are significantly lower than WT. Combined these data indicate that theTRPM1 channel in Gpr179 ${ }^{\text {nob5 }}$ rod DBCs has a low open probability that is similar to TRPM1 ${ }^{-/-}$rod DBCs where the channel is absent. The number of rod DBCs in each experimental group is shown within each bar of the histograms. 


\section{Long duration light increments evoke an ERG b-wave in Gpr179 nob5}

Since rod DBCs can response to strong pharmacological manipulation, we hypothesized that long duration full-field light flash stimuli also might evoke an ERG bwave. To test this we recorded ERGs from WT, Gpr179 ${ }^{\text {nob5 }}$ and $\operatorname{Trpm}^{-1-}$ mice using an LED-based stimulation system to present a $-1.2 \mathrm{log} \mathrm{cd} / \mathrm{m}^{2}$ stimulus for durations that ranged from 10 to $1000 \mathrm{msec}$. WT responses were dominated by the b-wave (Fig. 7A), which grew in amplitude with increasing stimulus duration (Fig. 7B). The same stimuli evoked a slow b-wave in Gpr179 $19^{\text {nob5 }}$ mice, whose amplitude also increased with stimulus duration. In contrast, none of the stimulus configurations evoked a b-wave in Trpm1 $1^{-1-}$ mice (Fig. 7) or in two other mouse models of cCSNB, Nyx $x^{\text {nob }}$ and Grm6 $6^{\text {nob3 }}$ (data not shown). Compared to WT, the amplitude of the response from the Gpr179 ${ }^{\text {nob5 }}$ retina was significantly smaller (two-way ANOVA; $P<0.001$ ). Whereas the WT response saturated to a $\sim 50$ msec stimulus duration, the $G p r 179^{\text {nob5 }}$ response continued to increase through stimulus durations of $\sim 500 \mathrm{msec}$ (Fig. 7). These ERG results are consistent with the mGluR6 mediated gating of TRPM1 we observe in the patch clamp recordings of Gpr179 ${ }^{\text {nob5 }}$ rod DBCs. 

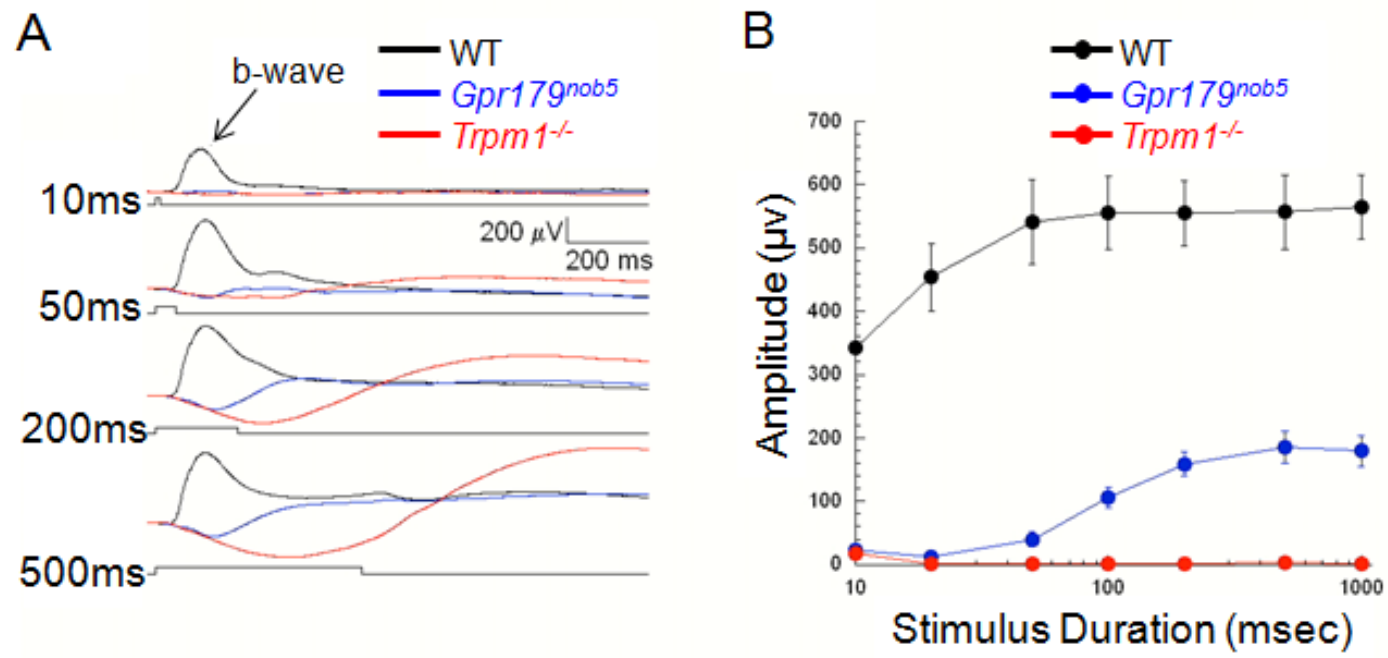

Figure 7. Increasing flash duration increases the ERG b-wave amplitude in Gpr179 ${ }^{\text {nob5 }}$ mice. (A) Representative ERG responses recorded from WT (black), Gpr179 $^{\text {nob5 }}$ (blue) and $\mathrm{Trpm}^{-/}$(red) mice to $-1.2 \mathrm{log} \mathrm{cd} / \mathrm{m}^{2}$ stimuli of increasing duration (top to bottom; and, indicated by the stimulus trace below each set of waveforms). Note that as stimulus duration increases, a slow positive wave becomes apparent in Gpr179 ${ }^{n o b 5}$ mice that is absent in $\operatorname{Trpm1} 1^{-/-}$mice. Waveforms indicate the average of: WT $(n=3), \operatorname{Gpr} 179^{\text {nob5 }}(n=12)$ and $\operatorname{Trpm}^{-/}(n=7)$ mice. (B) Average ( \pm sem) b-wave amplitude evoked by flash stimuli of different durations for the same mice. Note that the amplitude of the Gpr179 ${ }^{\text {nob5 }}$ response increases across a range of flash durations $(20-500 \mathrm{msec})$ whereas WT response amplitudes are stable $(\geq 20 \mathrm{msec})$, and $\operatorname{Trpm}^{-/-}$mice lack a response at any duration. 


\section{GPR179 and RGS7/11 provide unique functional roles in the DBC cascade}

Because the DBC dendritic tips of $G p r 179^{\text {nob5 }}$ mice lack expression of RGS7 and RGS11 (Orlandi et al 2012) and $R G S 7^{-/} / R G S 11^{-/}$DBCs also show very small light evoked responses (Cao et al 2012), it is possible that all of our observations result from the absence of the RGS7/11 complex. Because GPR179 is expressed and normally localized in $R G S 7^{-/} / R G S 11^{-/}$DBCs (Fig. 8A), we repeated our characterizations (Fig. 5 and 6) in $R G S 7^{-1 /} / R G S 11^{-/-}$rod DBCs and determined similarities and differences to differentiate the role of GPR179 from RGS7 and RGS11. The response amplitude evoked by $0.6 \mathrm{mM} 1 \mathrm{sec}$ CPPG puffs in $R G S 7^{-/} / R G S 11^{-/}$and $\mathrm{Gpr} 179^{\text {nob5 }}$ rod DBCs were similar (Fig. 8B; $P=0.45)$. In addition, both the standing outward current and current variance were similar in $R G S 7^{-/} / R G S 11^{-/}$and $G p r 179^{\text {nob5 }}$ rod DBCs (Fig. 8C-E, one way ANOVA: $P>.05$ ), and these parameters in the $R G S 7^{-/} / R G S 11^{-/}$cells were significantly lower than in WT cells (one way ANOVA: $P<0.0001 ; P<0.01$ ). We conclude that RGS7 and RGS11 are responsible for modulation of the sensitivity through the mGluR6 cascade.

When we tested the ability of capsaicin to directly open the TRPM1 channel in $R G S 7^{-/} / R G S 11^{-/-}$we found a surprising result. Unlike the significantly smaller capsaicin evoked response found in Gpr179 ${ }^{\text {nob5 }}$ rod DBCs (Fig. 5E), capsaicin evoked responses in $R G S 7^{-/} / R G S 11^{-/}$rod DBCs were similar in amplitude to WT (Fig. 8F,G; one-way

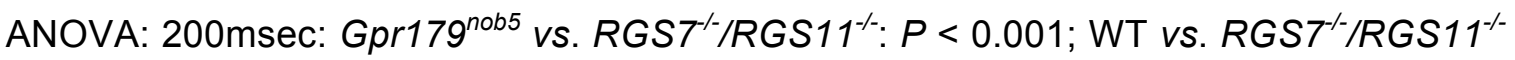
$: P>.05)$. These data suggest that $G P R 179^{-/-}$and $R G S 7^{-/ / R G S 11^{-/}}$double knockouts phenocopy each other for several characteristics, including response to CPPG, standing current and current variance. However, the presence of GPR179 even in the absence of RGS7 and RGS11 results in setting the "state" of TRPM1 such that the capsaicin response is similar to WT, presumably because of its direct interaction with TRPM1. 
A
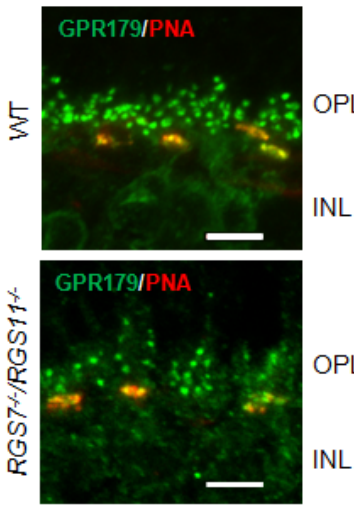

B

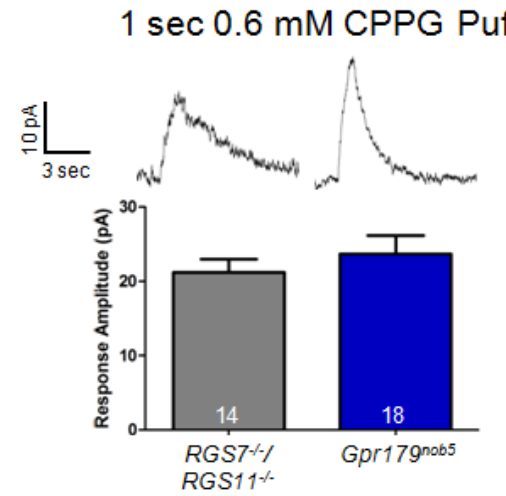

C
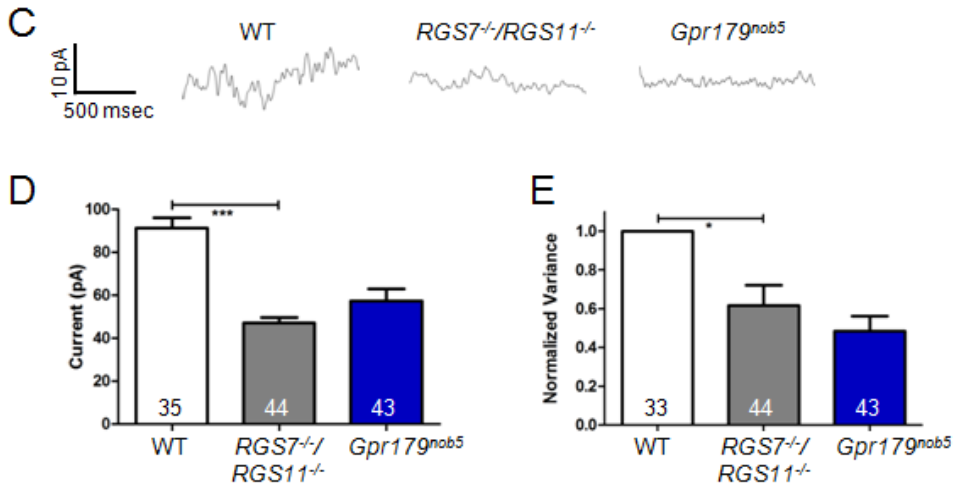

$\mathrm{F}$

G
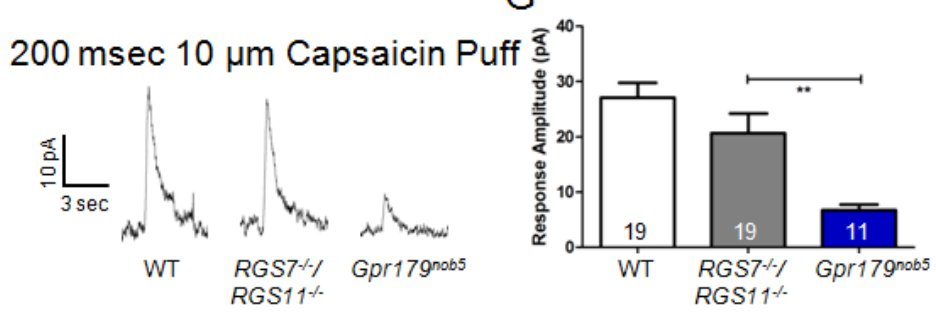

Figure 8. Capsaicin modulation of the TRPM1 channel differentiates the roles of Gpr179 ${ }^{\text {nob }}$ and $R G S 7^{-/} / R_{G S 11^{-/}}$in the mGluR6 signaling cascade. (A) Representative confocal images of retina sections from WT and $R G S 7^{-/} / R_{G S 11^{-/}}$ double knockouts labeled with antibodies to GPR179 and PNA (a cone terminal marker) show that GPR179 is localized normally in $R G S T^{/} / R G S 11^{-/}$retina. (B) Representative voltage clamp responses of $R G S 7^{-/} / R G S 11^{-/}$and $G p r 179^{n o b 5}$ rod DBCs evoked by a $1 \mathrm{sec}, 0.6 \mathrm{mM}$ CPPG puff. The histogram compares the response amplitudes and shows that $R G S 7^{-/} / R G S 11^{-/}$and $G p r 179^{n o b 5}$ are similar. (C) Representative traces from voltage clamp recordings of $\mathrm{WT}, \mathrm{RGS7}{ }^{-/} / \mathrm{RGS11}^{-/}$ and $G p r 179^{n o b 5}$ rod DBCs held at $+50 \mathrm{mV}$. Analyses are similar to those described in Figure 6. (D) Both standing current and (E) current variance are similar in RGST

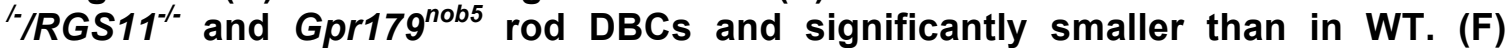
Representative voltage clamp responses WT, $R G S 7^{/ /} / R G S 11^{/-}$and $G p r 179^{n o b 5}$ rod DBCs to capsaicin puffs $(200 \mathrm{msec}$; $(10 \mu \mathrm{M})$. (G) Histograms compare peak response amplitudes for rod DBCs and show that WT and $R G S 7^{-/} / R_{G S 11^{-1}}$ are similar, whereas Gpr179nob5 rod DBC responses are significantly smaller. The number of rod DBCs in each experimental group is shown within each bar of the histograms. 


\section{Discussion}

Visual function in starlight depends on the modulation of mGluR6, which gates the TRPM1 channel in rod DBCs. While the last four years, have seen more growth in this area than the previous 40 , many details of the cascade mechanism remain poorly understood. Our results show that a new seven transmembrane protein, GPR179 along with RGS7/11 are required for maximal sensitivity of the cascade and that GPR179 is required for modulation of TRPM1 gating. Our immunohistological and protein biochemistry results support our functional assessments. GPR179 colocalizes with mGluR6 (Peachey et al., 2012b), TRPM1 and nyctalopin (Fig. 1) placing it in a prime location to be part of the gating mechanism controlling TRPM1 activity, and GPR179 and TRPM1 physically interact (Fig. 3).

At low light levels, the concentration of glutamate in the synaptic cleft is high and the mGluR6 receptor is maximally activated, which maximally activates a G-protein cascade that results in the exchange of $\mathrm{Ga}_{0}$-GDP to the active $\mathrm{Ga}_{0}$-GTP bound form, which releases the G $\beta y$ dimer. The exact mechanism of TRPM1 gating, and whether this is accomplished by $\mathrm{Ga}_{0}$-GTP or Gßy, is an active research area (Koike et al., 2010a; Koike et al., 2010b; Shen et al., 2012). Application of $\mathrm{G} \beta Y$ or activated $\mathrm{Ga}_{0}$ directly via the patch pipette to mouse rod DBCs in a retinal slice suggest that G $\beta y$ closes the channel while addition of activated Gao has minimal impact (Shen et al., 2012). These authors argue that G $\beta Y$ either directly or indirectly closes TRPM1 channels. This model predicts the light response deactivates $G \beta \gamma$, presumably by reformation of the trimeric $G$ protein complex. This would suggest that a large pool of $\mathrm{Ga}_{0}$-GDP must be available to rapidly reform the trimeric G-protein complex once Gßy ceases the interactions needed to inhibit TRPM1. This could explain why RGS7 and RGS11 are required and present in excess, indicated by the fact that a mouse without RGS11 and a hypomorphic allele of RGS7 had near normal ERG b-waves (Mojumder et al., 2009; Chen et al., 2010). A 
significant change in the ERG b-wave was only demonstrated when both genes were completely eliminated (Cao et al., 2012; Shim et al., 2012). The competing model of TRPM1 gating is that activated $\mathrm{Ga}_{0}$ closes the channel, and that the RGS proteins are required to rapidly inactivate $\mathrm{Ga}_{0}-\mathrm{GTP}$.

The Gßy model predicts that the standing inward current seen in DBCs reflects a balance between the constant activation of GBy due to tonic activation of mGluR6 and its inactivation by rebinding to $\mathrm{Ga}_{0}$-GDP. In the $G p r 179^{\text {nob5 }}$ mutants, which lack RGS7 and RGS11 at the DBC tips (Orlandi et al., 2012), and the $R G S 7^{-/} / R G S 11^{-/}$mutants, the model predicts an increase in active $G \beta y$ because there is reduced ability to hydrolyze GTP to GDP bound to $\mathrm{Ga}_{0}$. The consequence of this would be a decrease in TRPM1 open probability and in the DBC standing current. Under our experimental conditions of maximal activation of mGluR6, mimicking "dark" adapted conditions, we see both of these features, a dramatically decreased standing current and decreased open probability of TRPM1 in rod DBCs from Gpr179 ${ }^{\text {nob5 }}$ and $R G S 7^{-/} / R G S 11^{-/}$rod DBCs (Fig. 6 and Fig. 8). This effect is not the result of the absence of TRPM1 from the rod DBC signal transduction complex because; 1) immunohistochemistry shows TRPM1 is localized normally in Gpr179 ${ }^{n o b 5}$ and in $R G S 7^{-/} / R G S 11^{-/}$retinas (Fig. 1 and Cao et al. (2012)); 2) there are responses evoked in rod DBCs by: (a) long duration flashes in the ERG of Gpr179 nob5 mice and (b) high concentration/long application of CPPG in Gpr179 ${ }^{\text {nob5 }}$ rod DBCs (Fig. 5 and Fig. 8 B); and 3) activation of TRPM1 by capsaicin in Gpr179 nob5 rod DBCs. The rod DBCs of Gpr179 nob5 and $R G S 7^{-/} / R G S 11^{-/}$mice share many characteristics and one pivotal difference; the $R G S 7^{-/} / R G S 11^{-/}$DBCs have a normal response to capsaicin. There are two possibilities that could explain this result. First, there could be more TRPM1 channels present on the DBC dendrites. Second, the presence of GPR179, which interacts with TRPM1, could by a currently unknown mechanism set the "state" of the TRPM1 channel such that capsaicin is able to 
maximally activate the channel and that this is different than the requirements of gating by the mGluR6 cascade. We favor the latter hypothesis for several reasons. An apparently simple test of the first mechanism would be to quantify, using western blots, the level of TRPM1 in the GPR $179^{-/}$and the $R G S 7^{-/} / R G S 11^{-/}$retinas. However, this does not assess the amount of channel at the tips of the DBCs because TRPM1 is present in intracellular compartments throughout the DBCs (Fig. 1) and (Morgans et al., 2009; van Genderen et al., 2009; Cao et al., 2011; Pearring et al., 2011; Peachey et al., 2012a; Xu et al., 2012). The fact that the maximal CPPG response in Gpr179 ${ }^{\text {nob5 }}$ and the $R G S 7^{-/} / R G S 11^{-/}$rod DBCs is similar suggests similar levels of TRPM1 are linked to the mGluR6 cascade in these mutants. Therefore in the $R G S 7^{-/} / R G S 11^{-/}$, which express GPR179, we propose that the state of the TRPM1 channel is fundamentally different than in the GPR $179^{\text {nob5 }}$ DBCs and this allows capsaicin to activate it to normal levels. What this means at the molecular level is currently unclear but likely critical to understanding how TRPM1 is gated in DBCs.

Experiments described here and elsewhere show that the known components of the mGluR6/TRPM1 cascade are closely associated and many physically interact (Cao et al., 2011; Pearring et al., 2011; Orlandi et al., 2012). These interactions are likely to be critical to system performance and understanding the details of these interactions and how they likely change during light stimulation will be critical to a full understanding of the DBC signaling system. The importance of the physical arrangement of the cascade components is highlighted by the fact that RGS7 and RGS11 are still expressed in Gpr179 ${ }^{\text {nob5 }}$ mice, but are not localized correctly to the tips of the DBCs (Orlandi et al., 2012). There are likely to be many other proteins critical to complex function. One such molecule is LRIT3, a protein of unknown function, but when absent results in cCSNB in humans (Zeitz et al., 2013) with a phenotype consistent with disrupted DBC signal transduction. 
In conclusion, we show that GPR179 interacts with TRPM1 and one of its

functions is to localize RGS7 and RGS11 near the signaling complex (Orlandi et al., 2012). A second critical function is to set the "state" of TRPM1 such that it responds optimally to deactivation of the mGluR6 cascade. Understanding this "state" at the molecular level may provide important clues as to how TRPM1 is gated by the mGluR6 cascade.

References

Audo I et al. (2009) TRPM1 is mutated in patients with autosomal-recessive complete congenital stationary night blindness. Am J Hum Genet 85:720-729.

Audo I et al. (2012) Whole-Exome Sequencing Identifies Mutations in GPR179 Leading to Autosomal-Recessive Complete Congenital Stationary Night Blindness. Am J Hum Genet 90:321-330.

Boycott BB, Dowling JE, Kolb H (1969) Organization of the Primate Retina: Light Microscopy. Philosophical transactions of the Royal Society of London Series B, Biological sciences 255:109-184.

Cao Y, Posokhova E, Martemyanov KA (2011) TRPM1 forms complexes with nyctalopin in vivo and accumulates in postsynaptic compartment of ON-bipolar neurons in mGluR6-dependent manner. J Neurosci 31:11521-11526.

Cao Y, Pahlberg J, Sarria I, Kamasawa N, Sampath AP, Martemyanov KA (2012) Regulators of G protein signaling RGS7 and RGS11 determine the onset of the light response in ON bipolar neurons. Proc Natl Acad Sci U S A 109:7905-7910.

Chen FS, Shim H, Morhardt D, Dallman R, Krahn E, McWhinney L, Rao A, Gold SJ, Chen CK (2010) Functional redundancy of R7 RGS proteins in ON-bipolar cell dendrites. Invest Ophthalmol Vis Sci 51:686-693.

Dhingra A, Lyubarsky A, Jiang M, Pugh EN, Jr., Birnbaumer L, Sterling P, Vardi N (2000) The light response of ON bipolar neurons requires G[alpha]o. J Neurosci 20:9053-9058.

Dhingra A, Jiang M, Wang TL, Lyubarsky A, Savchenko A, Bar-Yehuda T, Sterling P, Birnbaumer L, Vardi N (2002) Light response of retinal ON bipolar cells requires a specific splice variant of Galpha(o). J Neurosci 22:4878-4884.

Dhingra A, Ramakrishnan H, Neinstein A, Fina ME, Xu Y, Li J, Chung DC, Lyubarsky A, Vardi $N$ (2012) Gbeta3 is required for normal light ON responses and synaptic maintenance. J Neurosci 32:11343-11355.

Ghosh KK, Bujan S, Haverkamp S, Feigenspan A, Wassle H (2004) Types of bipolar cells in the mouse retina. J Comp Neurol 469:70-82.

Gregg RG, Mukhopadhyay S, Candille SI, Ball SL, Pardue MT, McCall MA, Peachey NS (2003) Identification of the gene and the mutation responsible for the mouse nob phenotype. Invest Ophthalmol Vis Sci 44:378-384.

Gregg RG, Kamermans M, Klooster J, Lukasiewicz PD, Peachey NS, Vessey KA, McCall MA (2007) Nyctalopin expression in retinal bipolar cells restores visual function in a mouse model of complete X-linked congenital stationary night blindness. J Neurophysiol 98:3023-3033.

Jeffrey BG, Morgans CW, Puthussery T, Wensel TG, Burke NS, Brown RL, Duvoisin RM (2010) R9AP stabilizes RGS11-G beta5 and accelerates the early light response of ON-bipolar cells. Vis Neurosci 27:9-17. 
Kaneko A, Saito T (1983) lonic mechanisms underlying the responses of off-center bipolar cells in the carp retina. II. Studies on responses evoked by transretinal current stimulation. J Gen Physiol 81:603-612.

Koike C, Numata T, Ueda H, Mori Y, Furukawa T (2010a) TRPM1: A vertebrate TRP channel responsible for retinal ON bipolar function. Cell Calcium 48:95-101.

Koike C, Obara T, Uriu Y, Numata T, Sanuki R, Miyata K, Koyasu T, Ueno S, Funabiki K, Tani A, Ueda H, Kondo M, Mori Y, Tachibana M, Furukawa T (2010b) TRPM1 is a component of the retinal ON bipolar cell transduction channel in the mGluR6 cascade. Proc Natl Acad Sci U S A 107:332-337.

Li Z, Sergouniotis PI, Michaelides M, Mackay DS, Wright GA, Devery S, Moore AT, Holder GE, Robson AG, Webster AR (2009) Recessive mutations of the gene TRPM1 abrogate ON bipolar cell function and cause complete congenital stationary night blindness in humans. Am J Hum Genet 85:711-719.

Maddox DM, Vessey KA, Yarbrough GL, Invergo BM, Cantrell DR, Inayat S, Balannik V, Hicks WL, Hawes NL, Byers S, Smith RS, Hurd R, Howell D, Gregg RG, Chang B, Naggert JK, Troy JB, Pinto LH, Nishina PM, McCall MA (2008) Allelic variance between GRM6 mutants, Grm6nob3 and Grm6nob4 results in differences in retinal ganglion cell visual responses. J Physiol 586:4409-4424.

Masu M, Iwakabe H, Tagawa Y, Miyoshi T, Yamashita M, Fukuda Y, Sasaki H, Hiroi K, Nakamura $Y$, Shigemoto R, et al. (1995) Specific deficit of the ON response in visual transmission by targeted disruption of the mGluR6 gene. Cell 80:757-765.

McCall MA, Gregg RG (2008) Comparisons of structural and functional abnormalities in mouse b-wave mutants. J Physiol 586:4385-4392.

Miyake Y, Yagasaki K, Horiguchi M, Kawase Y, Kanda T (1986) Congenital stationary night blindness with negative electroretinogram. A new classification. Arch Ophthalmol 104:1013-1020.

Mojumder DK, Qian Y, Wensel TG (2009) Two R7 regulator of G-protein signaling proteins shape retinal bipolar cell signaling. J Neurosci 29:7753-7765.

Morgans CW, Brown RL, Duvoisin RM (2010) TRPM1: the endpoint of the mGluR6 signal transduction cascade in retinal ON-bipolar cells. Bioessays 32:609-614.

Morgans CW, Weiwei L, Wensel TG, Brown RL, Perez-Leon JA, Bearnot B, Duvoisin RM (2007) Gbeta5-RGS complexes co-localize with mGluR6 in retinal ONbipolar cells. Eur J Neurosci 26:2899-2905.

Morgans CW, Zhang J, Jeffrey BG, Nelson SM, Burke NS, Duvoisin RM, Brown RL (2009) TRPM1 is required for the depolarizing light response in retinal ON-bipolar cells. Proc Natl Acad Sci U S A 106:19174-19178.

Nawy S (2004) Desensitization of the mGluR6 transduction current in tiger salamander On bipolar cells. J Physiol 558:137-146.

Orlandi C, Posokhova E, Masuho I, Ray TA, Hasan N, Gregg RG, Martemyanov KA (2012) GPR158/179 regulate $G$ protein signaling by controlling localization and activity of the RGS7 complexes. J Cell Biol 197:711-719.

Pardue MT, McCall MA, LaVail MM, Gregg RG, Peachey NS (1998) A naturally occurring mouse model of $X$-linked congenital stationary night blindness. Invest Ophthalmol Vis Sci 39:2443-2449.

Peachey NS, Pearring JN, Bojang P, Jr., Hirschtritt ME, Sturgill-Short G, Ray TA, Furukawa T, Koike C, Goldberg AF, Shen Y, McCall MA, Nawy S, Nishina PM, Gregg RG (2012a) Depolarizing bipolar cell dysfunction due to a Trpm1 point mutation. J Neurophysiol 108:2442-2451.

Peachey NS et al. (2012b) GPR179 Is Required for Depolarizing Bipolar Cell Function and Is Mutated in Autosomal-Recessive Complete Congenital Stationary Night Blindness. Am J Hum Genet 90:331-339. 
Pearring JN, Bojang P, Jr., Shen Y, Koike C, Furukawa T, Nawy S, Gregg RG (2011) A role for nyctalopin, a small leucine-rich repeat protein, in localizing the TRP melastatin 1 channel to retinal depolarizing bipolar cell dendrites. J Neurosci 31:10060-10066.

Rood IM, Deegens JK, Merchant ML, Tamboer WP, Wilkey DW, Wetzels JF, Klein JB (2010) Comparison of three methods for isolation of urinary microvesicles to identify biomarkers of nephrotic syndrome. Kidney international 78:810-816.

Saito T, Kaneko A (1983) lonic mechanisms underlying the responses of off-center bipolar cells in the carp retina. I. Studies on responses evoked by light. J Gen Physiol 81:589-601.

Shen Y, Rampino MA, Carroll RC, Nawy S (2012) G-protein-mediated inhibition of the Trp channel TRPM1 requires the Gbetagamma dimer. Proc Natl Acad Sci U S A 109:8752-8757.

Shen Y, Heimel JA, Kamermans M, Peachey NS, Gregg RG, Nawy S (2009) A transient receptor potential-like channel mediates synaptic transmission in rod bipolar cells. J Neurosci 29:6088-6093.

Shim H, Wang CT, Chen YL, Chau VQ, Fu KG, Yang J, McQuiston AR, Fisher RA, Chen CK (2012) Defective retinal depolarizing bipolar cells (DBCs) in Regulators of Gprotein Signaling (RGS) 7 and 11 double null mice. J Biol Chem.

Slaughter MM, Miller RF (1983) An excitatory amino acid antagonist blocks cone input to sign-conserving second-order retinal neurons. Science 219:1230-1232.

van Genderen MM, Bijveld MM, Claassen YB, Florijn RJ, Pearring JN, Meire FM, McCall MA, Riemslag FC, Gregg RG, Bergen AA, Kamermans M (2009) Mutations in TRPM1 are a common cause of complete congenital stationary night blindness. Am J Hum Genet 85:730-736.

Xu Y, Dhingra A, Fina ME, Koike C, Furukawa T, Vardi N (2012) mGluR6 deletion renders the TRPM1 channel in retina inactive. J Neurophysiol 107:948-957.

Zeitz C et al. (2013) Whole-exome sequencing identifies LRIT3 mutations as a cause of autosomal-recessive complete congenital stationary night blindness. Am J Hum Genet 92:67-75. 


\title{
CURRICULUM VITAE
}

\author{
Thomas A. Ray
}

\section{Personal Data}

Work Address: $\quad$ University of Louisville

Dept. Biochemistry and Molecular Biology

A Building, Room 603

319 Abraham Flexner Way

Louisville, KY 40202

Phone 502-852-4687

E-mail taray001@louisville.edu

Date of Birth: $\quad$ March 4, 1986

Citizenship: $\quad$ U.S.A.

\section{Education}

2004-2008 Eastern Kentucky University, Richmond, KY.

Majors: Biology and Chemistry

2008-2013 University of Louisville, Louisville, KY. Department of Biochemistry and Molecular Biology Ph.D.

2014 Expected start date, January 6, 2014. Duke University, Durham, NC. Department of Neuroscience Postdoctoral Scholar

\section{Research Experience}

2005-2007 Principal Investigator: Eric E. Dueno 
Synthesized supramolecular molecules and obtained crystal structures for each. Research goal was to identify novel molecules with potential applications in medicinal host-guest chemistry.

2009-2013 Principal Investigator: Ronald Gregg

Identified and cloned the gene responsible for bipolar cell dysfunction (Gpr179) in a novel congenital stationary night blindness mouse model. Further characterized the protein to better understand the glutamate signaling cascade in depolarizing bipolar cells.

\section{Awards}

2008 Eastern Kentucky University Honors Program Scholar

$2012 \quad$ University of Louisville Neuroscience Day Graduate Student Poster First prize.

2012 ARVO Foundation/Retina Research Foundation/Joseph M. and Eula C. Lawrence Travel Grant

2013 Most Outstanding Graduate Student, University of Louisville School of Medicine Fall Commencement

2013 Graduate Dean's Citation, University of Louisville

\section{Societies}

ARVO (Association for Vision and Research in Ophthalmology)

\section{Invited Seminars}

$23^{\text {rd }}$ Annual Louisville Neuroscience Day. "GPR179, an orphan G proteincoupled receptor, is critical to rod mediated vision"

\section{Publications}

\section{Papers (peer reviewed)}

1. Dueno, E. E., Ray, T., Salvatore, R. N., Zambrano, C., Zeller, M. \& Hunter, A. D. (2007). 2,8,14,20-Tetrakis(4-hydroxyphenyl)pyrogallol[4]arene dimethyl- 
formamide hexasolvate, $\mathrm{C}_{52} \mathrm{H}_{40} \mathrm{O}_{16} \cdot 6 \mathrm{C}_{3} \mathrm{H}_{7} \mathrm{NO}$. Acta Cryst. E63, o3533-03534. doi:10.1107/S160053680702107

2. Eric E. Dueno, Allen D. Hunter, Matthias Zeller, Thomas A. Ray, Ralph N. Salvatore, Cesar H. Zambrano (2008). Crystal Structure of 2, 8, 14, 20- tert Butylpyrogallol[4]arene Journal of Chemical Crystallography 01/2008; 38(3):181187. DOI:10.1007/s10870-007-9285-7

3. Peachey NS, Ray TA, Florijn R, Rowe LB, Sjoerdsma T, Contreras-Alcantara S, Baba K, Tosini G, Pozdeyev N, luvone PM, Bojang P Jr, Pearring JN, Simonsz HJ, van Genderen M, Birch DG, Traboulsi El, Dorfman A, Lopez I, Ren H, Goldberg AF, Nishina PM, Lachapelle P, McCall MA, Koenekoop RK, Bergen AA, Kamermans M, Gregg RG. (2012). GPR179 is required for depolarizing bipolar cell function and is mutated in autosomal-recessive complete congenital stationary night blindness. Am J Hum Genet. 10;90:331-9. PMID: 22325362

4. Orlandi C, Posokhova E, Masuho I, Ray TA, Hasan N, Gregg RG, Martemyanov KA (2012). GPR158/179 regulate G protein signaling by controlling localization and activity of the RGS7 complexes. J Cell Biol. 2012 Jun 11;197(6):711-9. PMID:22689652.

5. Peachey NS, Pearring JN, Bojang P Jr, Hirschtritt ME, Sturgill-Short G, Ray TA, Furukawa T, Koike C, Goldberg AF, Shen Y, McCall MA, Nawy S, Nishina PM, Gregg RG. (2012). Depolarizing bipolar cell dysfunction due to a Trpm1 point mutation. J Neurophysiol. 2012 Nov;108(9):2442-51. doi: 10.1152/jn.00137.2012. PMID: 22896717

6. Jasmin Balmer, Rui Ji, Thomas A. Ray, Fabia Selber, Max Gassmann, Neal S. Peachey, Ronald G. Gregg, Volker Enzmann. (2013). Presence of the Gpr179 nob5 mutation in a $\mathrm{C} 3 \mathrm{H}$-derived transgenic mouse. Molecular Vision. 2013MOLVIS0459R: Accepted and ready for publication. 


\section{Manuscripts}

1. Thomas A. Ray*; Kathryn M. Heath*; Nazarul Hasan; Ivy S. Samuels; Kirill A. Martemyanov; Neal S. Peachey; Maureen A. McCall, Ronald G. Gregg. (2013). GPR179 is required for high sensitivity of the mGluR6 signaling cascade in depolarizing bipolar cells. Submitted Journal of Neuroscience, pending revisions.

\section{Book Chapters}

1. Ronald G. Gregg, Thomas A. Ray, Nazarul Hasan, Maureen A. McCall, Neal S. Peachey. Interdependence among members of the mGluR6 G-protein mediated signalplex of retinal depolarizing bipolar cells. Springer Vision Research. Submitted October 2013, Pending publication.

\section{Abstracts}

1. Thomas A. Ray, Maureen A. McCall, Ronald G. Gregg (2013). CACNA1S expression, structure and function in retinal depolarizing bipolar cells. Annual meeting of the Association for Research in Vision and Ophthalmology, Seattle, WA.

2. Thomas A. Ray, Ronald G. Gregg, Nazarul Hasan, Neal S. Peachey, Maureen A. McCall (2012). GPR179, an Orphan G protein-coupled receptor, is critical to depolarizing cell function. Federation of American Societies for Experimental Biology Retinal Neurobiology and Visual Processing meeting, Steamboat Springs, CO.

3. Thomas A. Ray, Nazarul Hasan, Maureen A. McCall, Gianluca Tosini, Patsy M. Nishina, Neal S. Peachey, Ronald G. Gregg (2012). GPR179, An Orphan G Protein-Coupled Receptor, Is Critical To Depolarizing Cell Function And Interacts With GRM6. Annual meeting of the Association for Research in Vision and Ophthalmology, Fort Lauderdale, FL.

4. Thomas A. Ray, Jillian N. Pearring, Pasano Bojang, Jr., Susana Contreras-Alcantara, 
Lucy Rowe, Gianluca Tosini, Patsy M. Nishina, Maureen A. McCall, Ronald G. Gregg, Neal S. Peachey (2011). Identification of a new mouse model of Complete Congenital Stationary Night Blindness. Annual meeting of the Association for Research in Vision and Ophthalmology, Fort Lauderdale, FL.

5. Thomas Ray, Dr. Eric E. Dueno. Synthesis of Functionalized Quinoxaline-Cavitands. Kentucky Academy of Science 2006 Annual Meeting, Morehead State University, Morehead, KY.

\section{Classroom Teaching}

2009-2010 Teaching assistant for Methods in Biochemistry and Molecular Biology

\section{Service Activities}

2010-2011 Department of Biochemistry and Molecular Biology new faculty search committee. 